\title{
Targeting the mitochondria for the treatment of MLH1-deficient disease
}

A thesis submitted in partial fulfillment of the requirements of the Degree of Doctor of Philosophy at the University of London

\author{
Dr. Sukaina Rashid
}

Clinical Research Fellow

Centre for Molecular Oncology

Barts Cancer Institute

Queen Mary University of London

EC1M 6BQ

UK 


\section{Declaration}

I, Sukaina Rashid confirm that the research included within this thesis is my own work or that where it has been carried out in collaboration with, or supported by others, that this is duly acknowledged below and my contribution indicated. Previously published material is also acknowledged below.

I attest that I have exercised reasonable care to ensure that the work is original, and does not to the best of my knowledge break any UK law, infringe any third party's copyright or other Intellectual Property Right, or contain any confidential material.

I accept that the College has the right to use plagiarism detection software to check the electronic version of the thesis.

I confirm that this thesis has not been previously submitted for the award of a degree by this or any other university.

The copyright of this thesis rests with the author and no quotation from it or information derived from it may be published without the prior written consent of the author.

Signature: 8raind

Date: 14/9/2016 


\section{Supervisors}

Primary Supervisor: Dr Sarah Martin

Senior Lecturer, Deputy Centre Lead,

Centre for Molecular Oncology, Barts Cancer Institute.

Secondary Supervisor: Dr Michelle Lockley

Clinical Senior Lecturer Centre Lead and Honorary Consultant in Medical Oncology, Centre for Molecular Oncology, Barts Cancer Institute.

\section{Collaborations}

\section{Professor Gyorgy Szabadkai}

Professor of Physiology

Cell and Developmental Biology, Division of Biosciences, University College London

\section{Professor Andrew Silver}

Professor of Cancer Genetics

Centre for Genomics and Child Health, Blizzard Institute, Queen Mary University of London

\section{Funding}

This work was supported by a grant from Cancer Research UK. 


\section{With special thanks}

First and foremost I would like to thank my PhD supervisor, Dr Sarah Martin, for taking me under her wing, guiding me from a complete scientific novice and facilitating my exploration of the wonderful world of mitochondria and mismatch repair. I am grateful for all of her advice and encouragement.

I would like to extend special thanks Dr Melissa Phillips, who has always been helpful and supportive, and without whose flexibility for covering my clinical commitments, I would not have been able to finish writing this thesis. I would like to extend my gratitude to my lab-mates, especially Dr Rumena Begum, Dr Gemma Bridge, Dr Delphine Guilotin, Dr Matthew Locke and Hannah Shailes. Thank you for being patient and encouraging - particularly at the beginning, where I am sure supervising my use of the equipment was like teaching a toddler calculus. A special thanks should also go to the other members of the molecular oncology department, especially $\mathrm{Dr}$ Dan Foxler, Ramsay Khadeir and Dr Katherine Bridge for all of their support and what I guess can only be described as "scientific banter," throughout my time in the lab.

To my comrades in arms, Dr Louise Lim, Dr Laura Tookman and Dr Kate Smith, thank you for helping me get through the tough times, and being there when we all needed to mutually despair at our ineptitudes.

Finally, I would like to extend my gratitude to Prof lan McNeish for believing in me, and introducing me to Dr Sarah Martin.

To my family, Mummy, Papa, and my sisters, Zahida and Zohra. Thank you for giving me everything l've ever needed, without ever having to ask for it.

To my Darling Husband, thank you for your unconditional love and support. Our first year of marriage has been overtaken by this $\mathrm{PhD}$ - thank you for believing in me, especially during the times when I didn't quite believe in myself. 


\section{Dedication}

I would like to dedicate this work to my parents, Shiraz and Noorjehan Rashid. Thank you for instilling the confidence and work ethic needed to achieve my ambitions. 


\section{Abstract}

The DNA Mismatch repair (MMR) pathway is responsible for the repair of base-base mismatches and insertion/deletion loops that arise during DNA replication. MMR deficiency is currently estimated to be present in $15-17 \%$ of colorectal cancer cases and $30 \%$ of endometrial cancers. MLH1 is one of the key proteins involved in the MMR pathway. MMR deficient tumours are often resistant to standard chemotherapies, therefore there is a critical need to identify new therapeutic strategies to treat MMR deficient disease. This study demonstrates that MLH1 deficient tumours are synthetically lethal with the mitochondrial-targeted agent Parthenolide which is known to induce reactive oxygen species (ROS) as one of its main mechanisms of action.

Upon functional analysis, I show for the first time that loss of MLH1 is associated with deregulated mitochondrial function evidenced by a reduction in complex I expression and activity, reduced basal oxygen consumption rate and reduced spare respiratory capacity. This mitochondrial phenotype in the MLH1-deficient cell lines is accompanied by a reduction in mitochondrial biogenesis as evidenced by down regulation of pgc1 $\beta$ and decreased mitochondrial copy number. Furthermore, MLH1-deficient cancer cells have a decreased antioxidant defence capacity with reduced expression of the antioxidant genes NRF1, NRF2, Catalase, Glutathione peroxidase and SOD1 as well as increased ROS production when treated with Parthenolide. I further demonstrate that both MSH2- and MSH6-deficient cell lines also display deficiencies in complex I compared to their MMR-proficient counterparts.

Taken together, the results of this study show a novel role for MLH1 in mitochondrial function and biogenesis. The MMR proteins $\mathrm{MSH} 2$ and $\mathrm{MSH} 6$ are also likely to have a role in the mitochondria. My results suggest that targeting the mitochondria may be a potential therapeutic strategy for the treatment of MMR and specifically MLH1 deficient disease. 


\section{Table of contents}

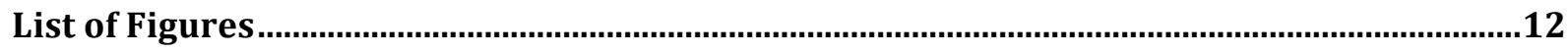

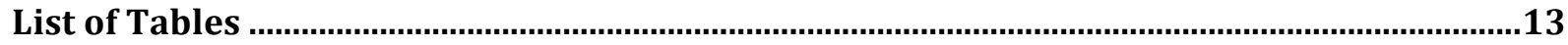

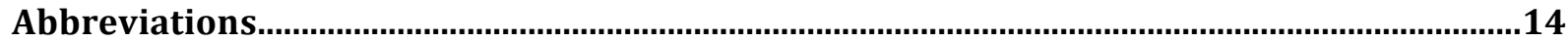

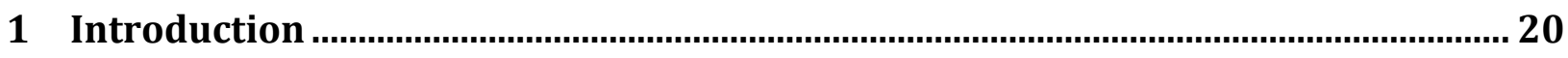

1.1 DNA damage

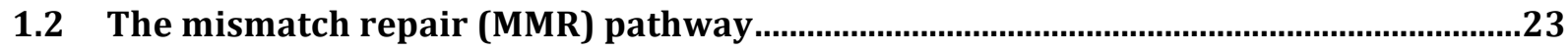

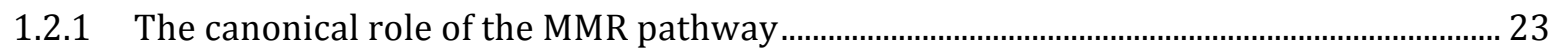

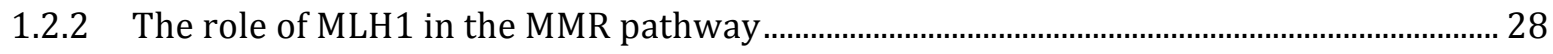

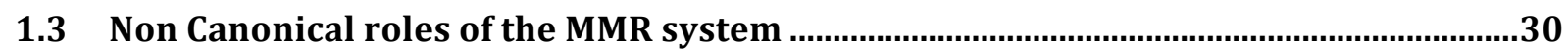

1.3.1 Meiotic and mitotic recombination and MMR …................................................................... 30

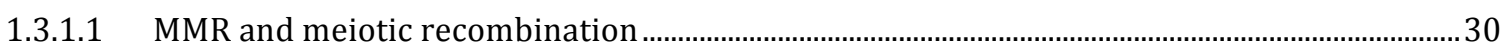

1.3.1.2 MMR and mitotic recombination.....................................................................................................

1.3.2 MMR and immunoglobulin diversification .............................................................................. 31

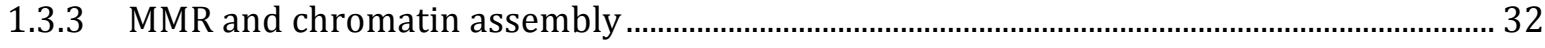

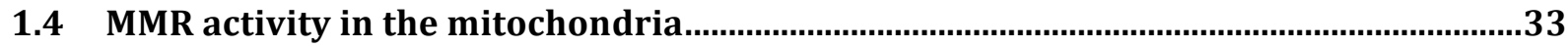

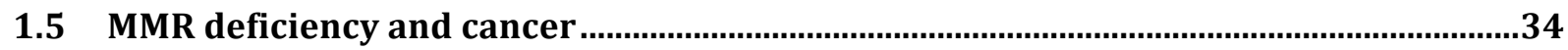

1.5.1 Treatment of MMR cancers

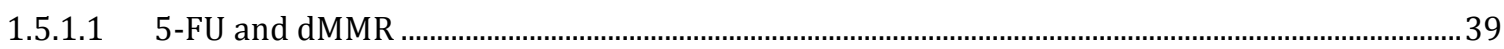

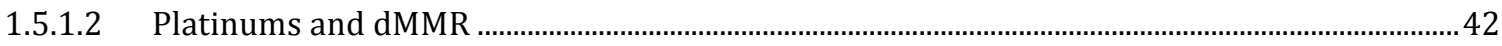

1.5.1.3 Topoisomerase inhibitors and anthracyclines in dMMR ........................................................43

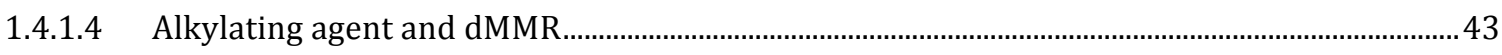

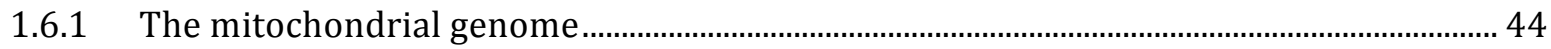

1.6.2 Heteroplasmy and homoplasmy ………….............................................................................. 44

1.6.3 Oxidative phosphorylation................................................................................................. 45

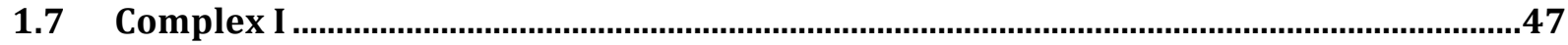

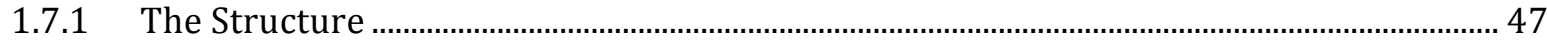

1.8.2 Electron transfer, proton translocation and the link between these processes ............. 52

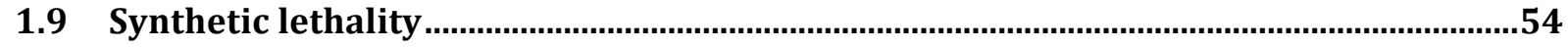

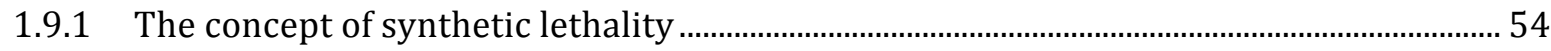

1.10.2 Synthetic lethality and the MMR pathway.......................................................................... 54

1.10.3 Synthetic lethality and cancer cell metabolism .............................................................. 55

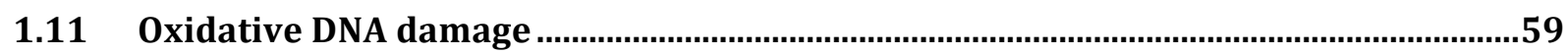

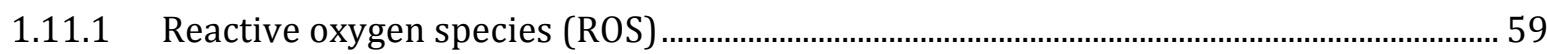




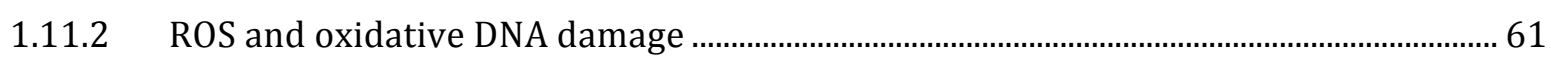

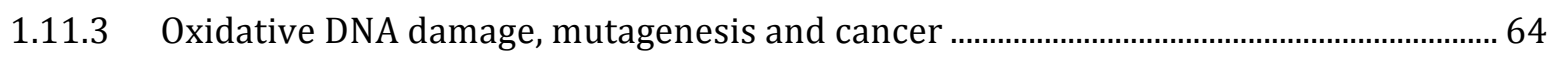

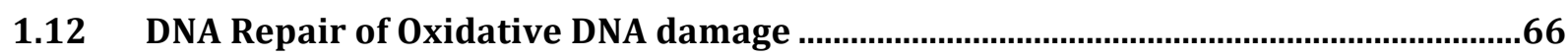

1.12.1 Base excision repair (BER) repair of nuclear oxidative DNA damage.............................. 66

1.12.2 Nucleotide excision repair (NER) and nuclear oxidative DNA damage ........................... 67

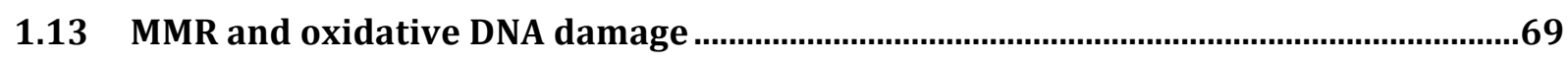

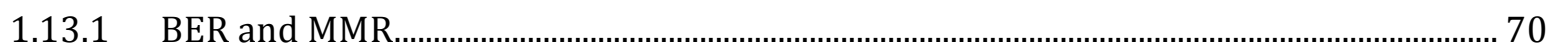

1.13.2 MMR and oxidative damage: relevance to carcinogenesis ................................................ 71

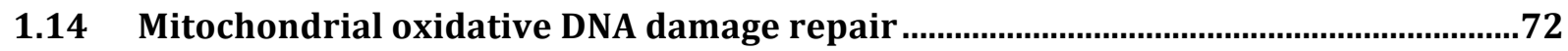

1.14.1 Repair of mitochondrial oxidative DNA damage by BER and NER ..................................... 72

1.14.2 DNA break repair in the mitochondria ……........................................................................ 73

1.14.3 Double strand break repair in the mitochondria................................................................... 73

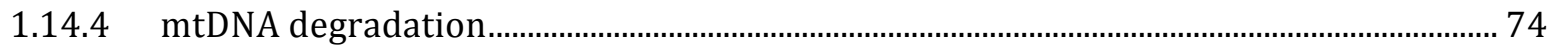

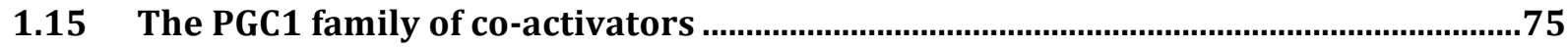

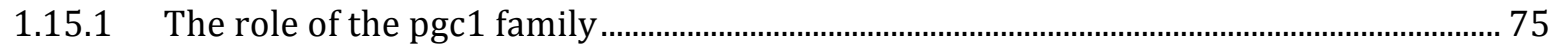

1.15.2 Mitochondrial biogenesis and the PGC1 - family ………………………………………... 75

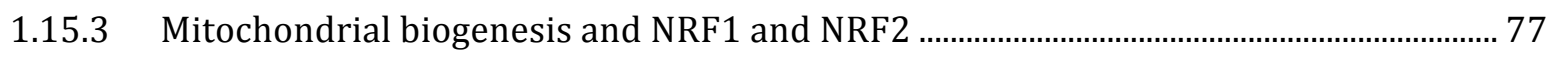

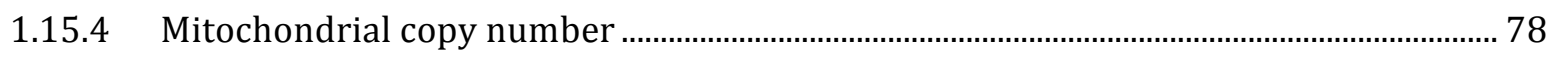

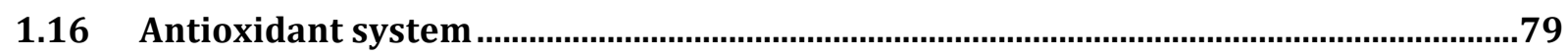

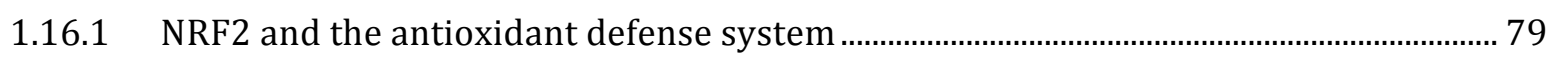

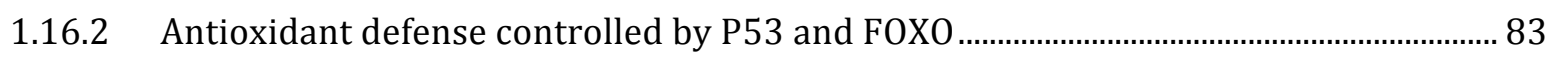

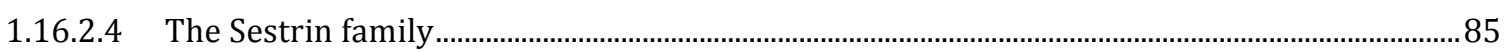

1.16.3 The role of PGC1 - family as antioxidants ............................................................................. 85

2 Aims and objectives ...................................................................................................... 86

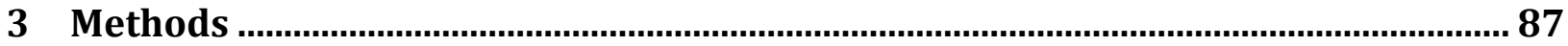

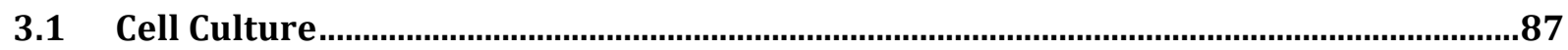

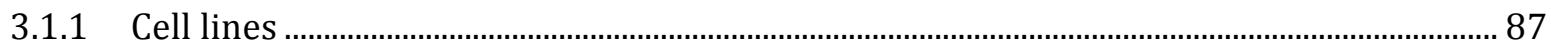

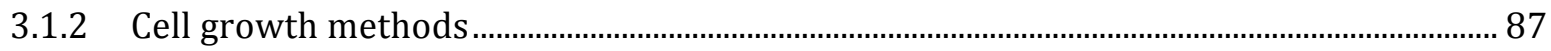

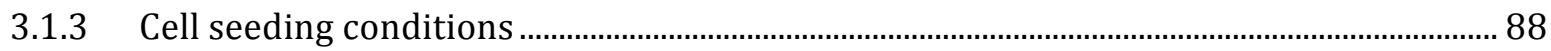

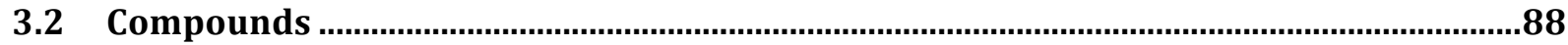

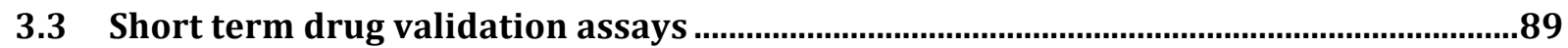

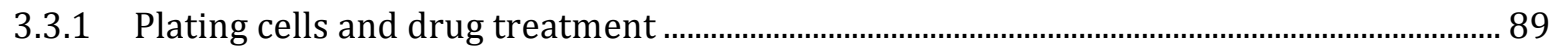

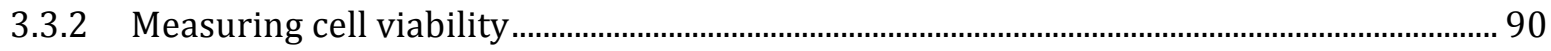

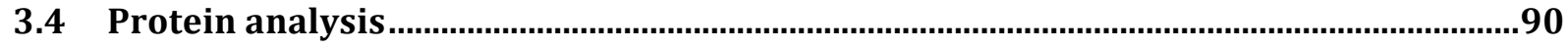

3.4.1 Preparation of protein lysates ……………............................................................................ 90 


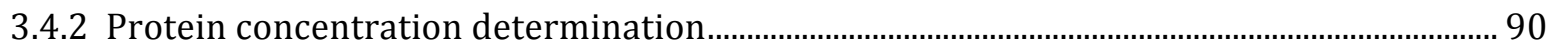

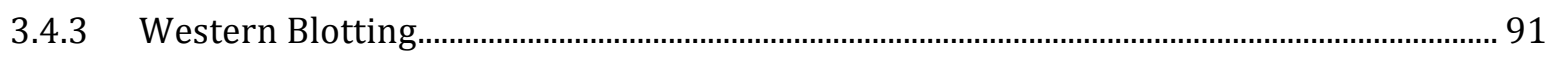

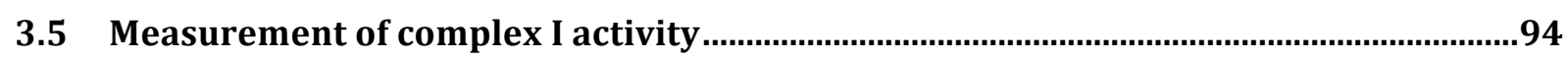

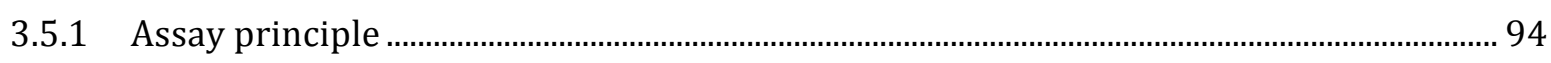

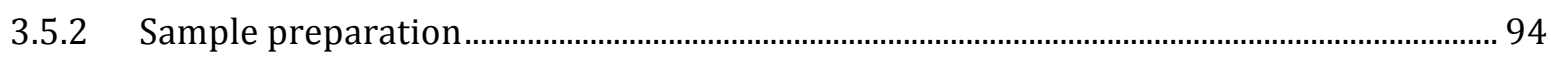

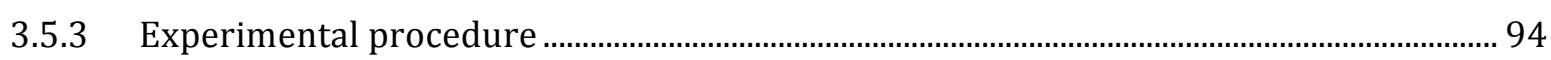

3.6 Quantitative reverse transcriptase-polymerase chain reaction (qRT-PCR) ...............95

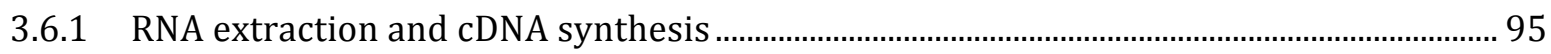

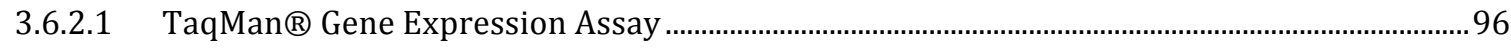

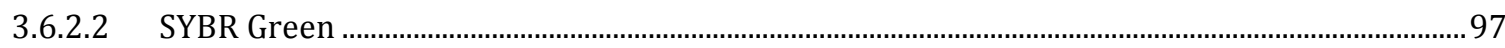

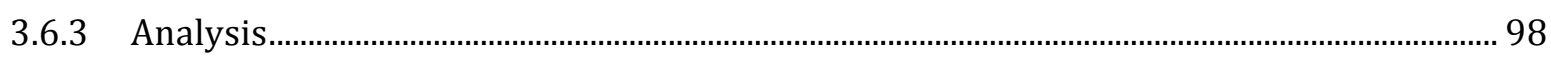

3.7 Measurement of oxidative consumption rate (OCR) and spare respiratory capacity 99

3.7.1 Day 1: cell plating ................................................................................................................ 99

3.7.2 Day 1: Preparing calibration plate ...................................................................................... 100

3.7.3 Day 2: preparing the cell culture plate.................................................................................101

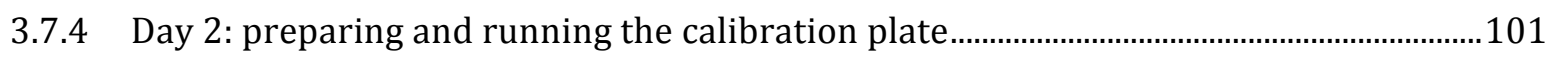

3.7.5 Running the cell culture plate ............................................................................................ 104

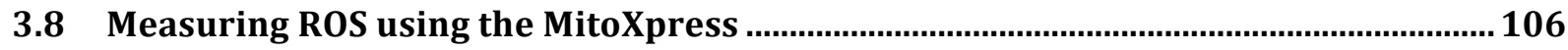

3.9 Sequencing the mitochondrial genome using the Illumina ${ }^{\circledR}$ Miseq ${ }^{\mathrm{TM}}$ platform ...... 107

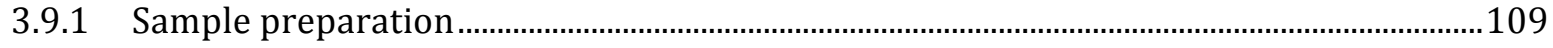

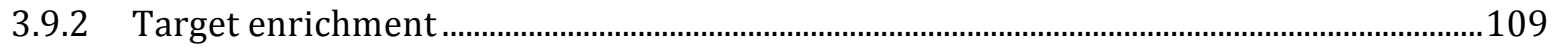

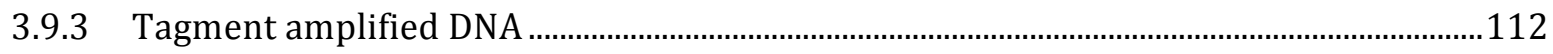

3.9.4 Limited-Cycle PCR Amplification ………….........................................................................113

3.9.5 Limited-Cycle PCR clean-up.................................................................................................116

3.9.6 Bead Based Normalization .................................................................................................

3.9.7 Pool and load from bead-based normalization .....................................................................118

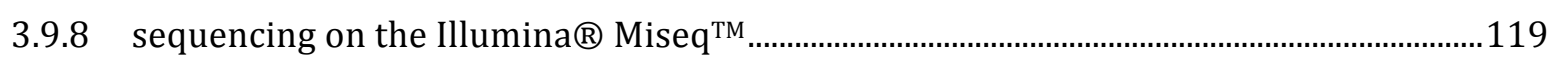

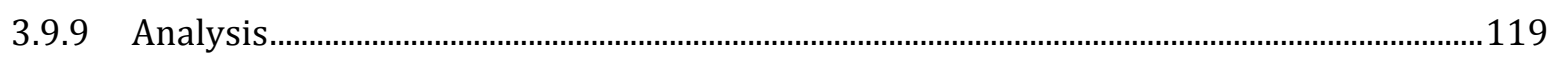

3.10 Measuring mitochondrial copy number changes .....................................................119

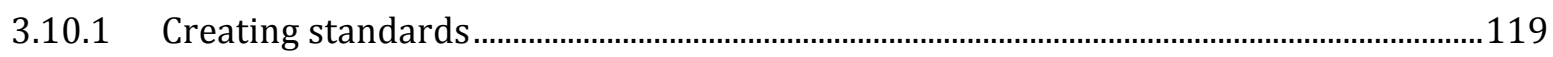

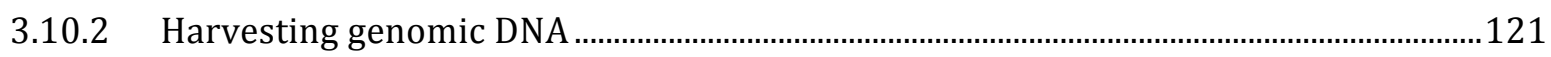

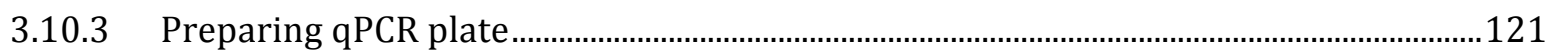

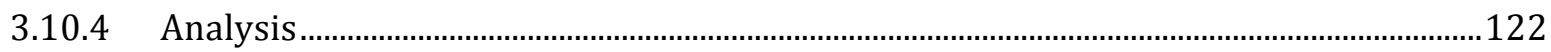

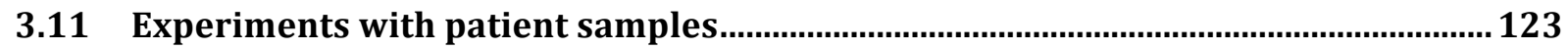

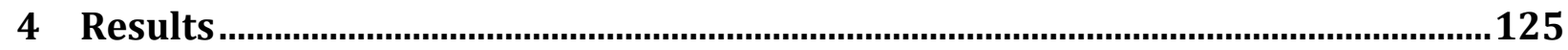




\subsection{Identification and characterization of novel therapeutics for the treatment of MLH1-}

deficient cancers

4.1 Validation of mitochondrial-targeted agents in a range of MLH1-deficient and proficient cell lines.

4.1.1 The ROS inducing agent Parthenolide is selectively lethal with MLH1-deficient cell lines.... 126

4.1.2 Investigating the mechanism behind the synthetic lethal relationship between

Parthenolide treatment and MLH1 loss. 140

4.1.2.1 Increased ROS production in MLH1-deficient cell lines treated with Parthenolide 140

4.1.2.2 MLH1-deficient cell lines do not have a synthetic lethal relationship with other ROS inducing agents.

\subsection{Functional investigation to study the differences between mitochondrial function in}

MLH1-deficient and proficient cells lines 149

4.2.1 Investigating the antioxidant defense system in MLH1 deficiency 149

4.2.1.1 Decreased gene and protein expression of the transcription factor NRF2 and the downstream antioxidant defense enzymes

4.2.2.1 Decreased Complex I expression in MLH1-deficient cell lines .....................................................153

4.2.2.2 Decreased activity of Complex I in MLH1-deficient cell lines ....................................................... 157

4.2.3 Investigating Oxidative phosphorylation upon MLH1 deficiency.......................................160

4.2.3.1 MLH1-deficient cell lines have decreased oxidative phosphorylation 160

4.2.4 Investigating differences in the mitochondrial genome between MLH1-proficient and deficient cell lines.

4.2.4.1 There are no significant differences in mutations within Complex I genes in MLH1-deficient and proficient cell lines.

4.2.5 Investigating mitochondrial biogenesis in MLH1-deficient cell lines ...... 165

4.2.5.1 Decreased mitochondrial biogenesis in MLH1-deficient cell lines.. 165

4.3 Validating experimental results in patient samples

4.3.1 Analysis of expression of antioxidant defense enzymes in MLH1-deficient tumours from patient samples 169

4.3.1.1 There is no significant decrease in expression of antioxidant defense genes in MLH1deficient and MSI patient tumour samples.

4.3.2 Analysis of mitochondrial phenotypes in other MMR-deficient cells Examining ..........176

4.3.2.1 Deficiencies in Complex I in MSH2 and MSH6 deficient cell lines. 176

4.4.2.2 MSH2 deficient cell lines have decreased mitochondrial copy number and decreased expression of the antioxidant defense enzymes.

5 Discussion 186

5.1 Identification and characterization of novel therapeutics for the treatment of MLH1 deficient cancers 
5.1.1 Validation of mitochondrial-targeted agents in a range of MLH1-deficient and proficient cell lines.

5.1.2 The ROS inducing agent Parthenolide is selectively lethal with MLH1-deficient cell lines 187

\subsection{Investigating the mechanism behind the synthetic lethal relationship between}

Parthenolide treatment and MLH1 loss

5.2.1 Increased ROS production in MLH1-deficient cell lines treated with Parthenolide .....188

5.2.2 MLH1-deficient cell lines do not have a synthetic lethal relationship with other ROS

inducing agents

\subsection{Functional Investigation to study the differences between mitochondrial function in}

MLH1-deficient and proficient cell lines.

\subsubsection{Investigating the antioxidant response in MLH1 deficient and proficient cell lines} 190

5.3.2 Investigating Complex I in MLH1 deficiency 192

5.3.2.1 Complex I and MMR deficient cancer cell lines.

5.3.3 MLH1 deficient cell lines have decreased basal OCR and spare respiratory capacity.194

5.3.3.1 Respiratory chain complex deficiencies in cancer. 195

5.3.4 Investigating differences in the mitochondrial genome between MLH1-proficient and deficient cell lines. 196

5.3.5 Investigating mitochondrial biogenesis in MLH1-deficient cell lines 198

5.3.5.1 The significance of mitochondrial copy number changes in cancer 200

5.3.5.2 Some cancers upregulate respiratory genes to compensate for decreased mitochondrial copy number

$5.4 \quad$ Validating experimental results in patient samples ..............................................................203

5.5 Analysis of mitochondrial phenotypes in other MMR-deficient cells ...................................204

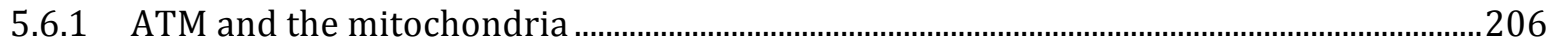

5.6.2 DNA repair proteins and nucleus-to-mitochondria signalling ……………..........................207

5.7 ROS induction and targeting the redox capacity of cancer cells .......................................209

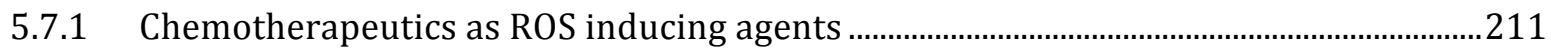

5.7.1.1 The quinones as ROS inducing agents............................................................................................ 211

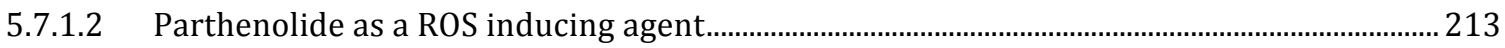

5.7.2 Drugs inhibiting the antioxidant response .......................................................................215

5.7.3 Synthetic lethal targeting of cancer cells with DNA repair deficiencies and ROS

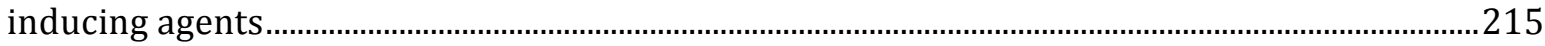

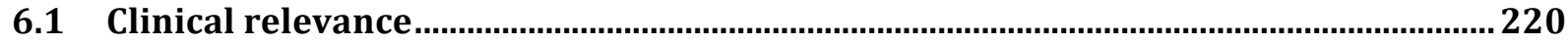

7.1 Conclusions

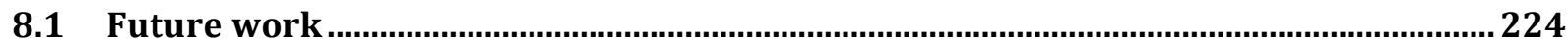


Bibliography.

Appendix 246

Appendix 1: Results for mitochondrial sequencing of MLH1 deficient (HCT116) and proficient cell lines (HCT116+chr3) 246

\section{List of Figures}

Figure 1.1 The DNA repair pathway and DDR proteins recruited depends on the type of DNA lesions present..................................................................................................... 22

Figure 1.2. Schematic of the MMR system. ................................................................ 27

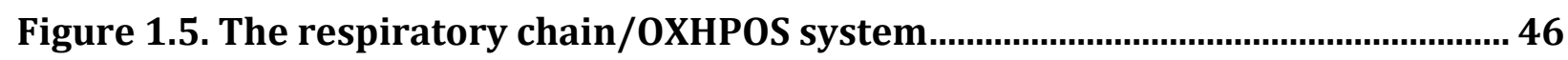

Figure 1.3: Accumulation of oxidative DNA damage causes synthetic lethality in

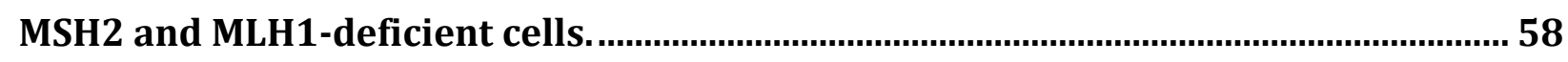

Figure 1.6. NRF2 regulates a multitude of antioxidant pathways................................. 82

Figure 4.2. The ROS inducing agent Parthenolide is synthetically lethal with the MLH1-deficient cell line HCT116 compared to its isogenetically matched MLH1proficient cell line.

Figure 4.3. The ROS inducing agent Parthenolide is synthetically lethal with MLH1deficient cell lines in a panel of cell lines from a range of tumour types.

4.1.1.2 MLH1-deficient cell lines are only synthetically lethal with Parthenolide and not other drugs targeting mitochondrial function. .135

Figure 6.1. (A) MLH1-deficient cell lines can be exploited clinically due to an attenuated antioxidant response.

Figure 7.1 MLH1-deficient cell lines display a complex mitochondrial phenotype..223 


\section{List of Tables}

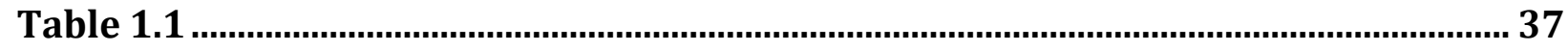

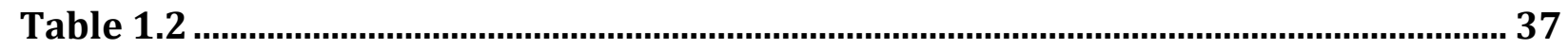

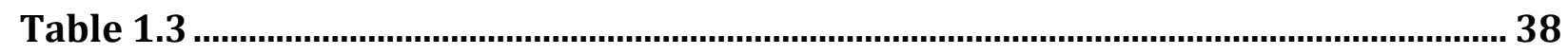

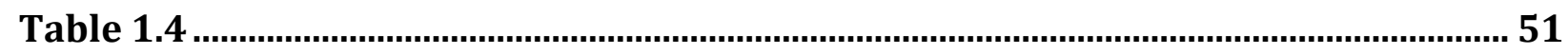

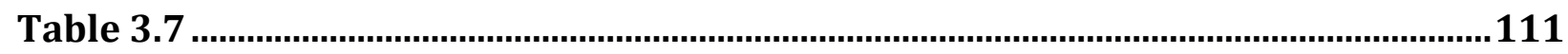

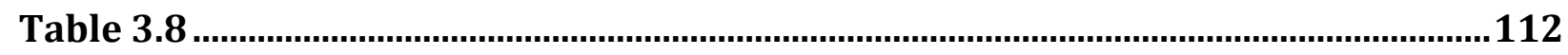

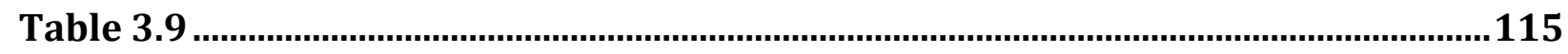

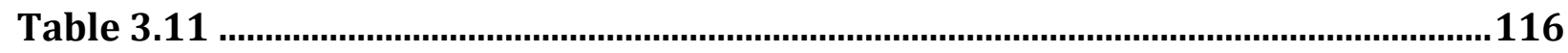

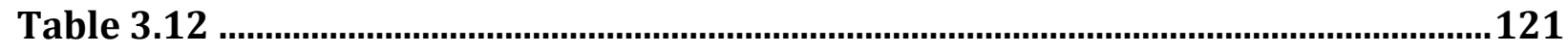

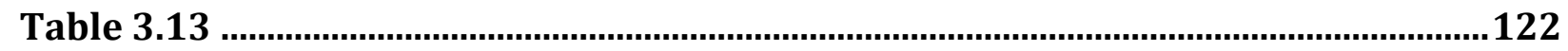

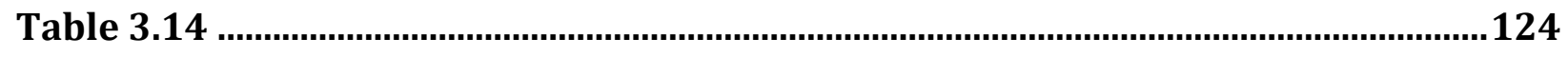

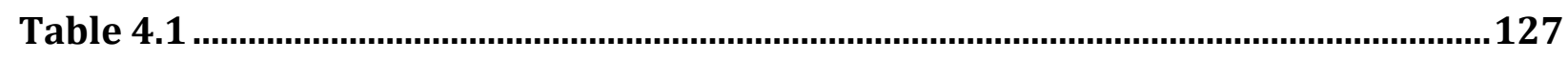




\section{Abbreviations}

\begin{tabular}{|c|c|}
\hline $\mathrm{O}_{2} \bullet-$ & Superoxide anions \\
\hline $\mathrm{HO} \bullet$ & Hydroxyl radical \\
\hline 2DG & 2-deoxy-D-glucose \\
\hline $5-\mathrm{FU}$ & 5 -fluorouracil \\
\hline 8-oxodG & 8-Oxo-2'-deoxyguanosine \\
\hline ADP & Adenosine diphosphate \\
\hline AID & Activation-induced deaminase \\
\hline AMPK & AMP-activated kinase \\
\hline ANT & Adenine nucleotide translocase \\
\hline AP & Apurinic/apyrimidinic \\
\hline APE1 & AP endonuclease 1 \\
\hline ARE & Antioxidant response element \\
\hline ASS1 & Argininosuccinate synthase 1 \\
\hline ATM & Ataxia telangiectasia mutated \\
\hline ATP & Adenosine triphosphate \\
\hline ATR & Ataxia telangiectasia and Rad3-related protein \\
\hline $\mathrm{BAX}$ & BCL2-associated $X$ protein \\
\hline $\mathrm{Bcl} 2$ & B-cell lymphoma 2 \\
\hline BER & Base excision repair \\
\hline BRCA1 & Breast Cancer type 1 susceptibility protein \\
\hline BRCA2 & Breast Cancer type 2 susceptibility protein \\
\hline BSA & Bovine serum albumin \\
\hline cDNA & Complementary DNA \\
\hline Chk1 & Checkpoint kinase 1 \\
\hline Chk2 & Checkpoint kinase 2 \\
\hline Chr & Chromosome \\
\hline CIMP & CpG island methylator phenotype \\
\hline Coenzyme Q & Ubiquinone \\
\hline Complex I (Cl) & NADH-ubiquinone oxidoreductase \\
\hline
\end{tabular}


Complex II (CII) Succinate dehydrogenase

Complex III (CIII) Succinate- quinone oxidoreductase

(cytochrome bc1)

Complex IV(CIV) cytochrome c oxidase,

Complex V (CV) F1FO-ATP synthase

CRC

Colorectal cancer

CSA

Cockayne syndrome protein $A$

CSB

Cockayne syndrome proteins $B$

CUL3

Cullin 3

CYPD

Cyclophillin D

D-loop

Non-coding displacement-loop

DDR

DNA damage response

DFS

Disease free survivial

DLC

Delocalized lipophilic cations

DMEM

Dulbecco's modified Eagle's medium

dMMR

MMR deficiency

DMSO

Dimethyl sulfoxide

DNA

Deoxyribonucleic acid

DNA pol

DNA polymerase

dNTP

Deoxyribonucleotide

DSB

Double strand break

E. coli

Escherichia coli

EDTA

Ethylenediaminetetraacetic acid

EGFR

Epidermal growth factor receptor

ELISA

Enzyme linked immunosorbent assay

ERRa

Estrogen related receptor $\alpha$

ETC

Electron transport chain

EXO1

Exonuclease 1

FAD

Flavin adenine dinucleotide

FapyGua

2,6-diamino-4-hydroxy-5-formamido-

pyrimidine

FBS

Fetal bovine serum

FdUMP

Fluoroodeoxyuridine monophosphate

FdUTP

Fluorodeoxyuridine triphosphate 


\begin{tabular}{|c|c|}
\hline FEN1 & Flap endonuclease 1 \\
\hline FMN & Flavin mononucleotide \\
\hline FOLFIRI & 5-fu + Oxaliplatin+Irinotecan \\
\hline FOLFOX & 5-fu+Oxaliplatin \\
\hline FOXO & Forkhead box $\mathrm{O}$ \\
\hline GCL & Glutamine cysteine ligase \\
\hline GCLC & Glutamine cysteine ligase catalytic \\
\hline GCLM & Glutamine cysteine ligase modifier \\
\hline GGR-NER & Global genome repair-Nucleotide excision repair \\
\hline GPx1 & Glutathione peroxidase 1 \\
\hline GPX2 & Glutathione-s-transferase \\
\hline GSH & Glutathione \\
\hline GSTM1 & Glutathione-s-transferase \\
\hline H. pylori & Helicobacter pylori \\
\hline $\mathrm{H}_{2} \mathrm{O}_{2}$ & Hydrogen peroxide \\
\hline HK & Hexokinase \\
\hline HMOX1 & Heme oxygenase 1 \\
\hline HNF4alpha & Hepatic nuclear receptor 4alpha \\
\hline HNPCC & Hereditary nonpolyposis colon cancer \\
\hline $\mathrm{HR}$ & Homologous recombination \\
\hline NHEJ & Non-homologous end joining \\
\hline IDL & Insertion/ deletion loop \\
\hline $\mathrm{IHC}$ & Immunohistochemistry \\
\hline IMS & Intermembrane space \\
\hline JAK2 & Janus kinase 2 \\
\hline KEAP1 & Kelch-like ECH associated protein 1 \\
\hline LIG1 & DNA ligase 1 \\
\hline LKB1 & Liver kinase B1 \\
\hline LP-BER & Long patch-base excision repair \\
\hline LV & Leucovorin \\
\hline MEF & Mouse embryonic fibroblast \\
\hline MGMT & O6-methylguanine DNA methyltransferase \\
\hline $\mathrm{mM}$ & milimolar \\
\hline
\end{tabular}


MMEJ

MMP

MMR

MNNG

MOMP

MPM

MSI

MSI-H

MSI-L

MSS

mtDNA

mTOR

mtTFB

MUTYH

NAC

NADPH

NEIL1

NEIL2

NEIL3

NER

NER

NHEJ

nM

$\mathrm{NO}$

NQO1

NRF1

NRF2 (pg)

NRs

NSCLC

NTHI

$\mathrm{O}_{2}$

${ }^{1} \Delta_{\mathrm{g}} \mathrm{O}_{2}$
Microhomolgy-mediated end-joining

Mitochondrial membrane potential

DNA mismatch repair

$\mathrm{N}$-methyl-N'-nitro-N-nitrosoguanidine

Mitochondrial outer membrane potential

Malignant pleural mesotheliomas

Microsatellite instability

High microsatellite instability

Low microsatellite instability

Microsatellite stable

Mitochondrial DNA

Mammalian target of rapamycin pathway

Mitochondrial transcription factor $B$

MutY homolog

$\mathrm{N}$-acetylcysteine

Nicotinamide adenine dinucleotide phosphate

$\mathrm{NEI}$ endonuclease VIII-like 1

$\mathrm{NEI}$ endonuclease VIII-like 2

$\mathrm{NEI}$ endonuclease VIII-like 3

Nucleotide excision repair

Nucleotide excision repair mismatch repair

Non-homologous end joining nucleotide

excision repair

Nanomolar

Nitric oxide

NADPH:quinone oxidoreductase 1

Nuclear factor 1

Nuclear factor erythroid 2-related factor 2

Nuclear receptors

Non-small cell lung cancer

Endonuclease three homolog 1

Oxygen

Singlet oxygen 
O6meG

OCDL

OGG1

ONOO-

$\mathrm{ONOOH}$

OS

$P$

PARP

PBR

PBS

PCNA

PDK

PI3K

PIN

PINK1

Polß

PRDX1

PTEN

PTPC

RFC

RFC

RNA

RNAPII

$\mathrm{RO} \mathrm{O}^{\circ}$

$\mathrm{RO}_{2}{ }^{\circ}$

ROS

RPA

RPMI

RR

S-phase

S. cerevisiae

SEM

SiRNA

SLC7A11
O6-methylguanine

Oxidatively induced clustered lesions

8-Oxoguanine glycosylase

Peroxynitrite

Peroxynitrous acid

Overall survival

$P$-value

Poly(ADP-ribose) polymerase 1

Peripheral benzodiazepine receptor

Phosphate buffered saline

Proliferating cell nuclear antigen

Pyruvate dehydrogenase kinase

Phosphoinositide 3 kinase

Prostate intraepilethial neoplasia

PTEN-induced putative kinase 1

DNA polymerase beta

Peroxiredoxin 1

Phosphatase and tensin homolog

Permeability transition pore complex

Replication factor $\mathrm{C}$

replication factor $\mathrm{C}$

Ribonucleic acid

RNA polymerase II

Alkoxyl radicals

peroxyl radicals

Reactive oxygen species

Replication protein A

Roswell Park Memorial Institute medium

Ribonucleotide reductase

Synthesis phase

Saccharomyces cerevisiae

Standard error of mean

Small interfering RNA

Solute carrier family 7 member 11 
SOD

SOD1

SOD2

SP-BER

SSB

SSB

SSDNA

TC-NER

TCA

TCR-NER

TDP1

TFAM

TFB1M

TFB2M

TFs

TLS

TMZ

TXN1

TXNRD

UNG

VDAC

YB1

YY1

$\mu \mathrm{g}$

$\mu \mathrm{M}$
Superoxide dismutase

$\mathrm{Cu}, \mathrm{Zn}-\mathrm{SOD}$

Mn-SOD

Short patch-base excision repair

Single strand break

Single strand break

Single stranded DNA

Transcription-coupled NER

Tricarboxylic acid cycle

Transcription coupled repair--Nucleotide

excision repair

Tyrosyl-DNA phosphodiesterase 1

Transcription Factor A

Mitochondrial transcription factor $B$ isoform 1

Mitochondrial transcription bactor $\mathrm{B}$ isoform 2

Transcription factors

Translesional synthesis

Temozolomide

Thioredoxin 1

Thioredoxin reductase

Uracil-DNA glycosylase

Voltage-dependent anion channel

Y-BOX binding protein

yin yang 1 transcription factor

Microgram

Micromolar 


\section{Introduction}

\subsection{DNA damage}

One of the key features of most tumours is genomic instability resulting in a multitude of different ways in which our DNA is altered ranging from nucleotide substitutions, insertions, deletions and changes in chromosomal copy number and structure [1]. These genomic changes occur as a result of continuous exposure to exogenous and endogenous sources of DNA damage. To combat this problem a complex system of several different mechanisms has evolved to repair DNA damage depending on the type of lesion present. As a whole, this complex network of pathways is collectively known as the DNA damage response (DDR). It has been estimated that in excess of 150 proteins are involved in the DDR and control of cell-cycle checkpoints to allow DNA repair. It is therefore not surprising that if mutations occur within these DDR genes, this would lead to carcinogenesis. There is a considerable body of research ongoing into understanding and developing novel strategies to target the DDR as a therapeutic option for a wide variety of cancers [2].

Exogenous DNA damage is caused by substances including cigarette smoke, industrial chemicals, mustard gases and chemotherapeutic drugs. Chemotherapeutic agents achieve cell death by causing lesions such as interstrand crosslinks (platinum containing agents), base alkylation (Temozolomide), single strand and double strand breaks (SSBs and DSBs, respectively) (Etoposide). Endogenous DNA damage includes damage caused by reactive oxygen species (ROS) resulting in oxidative DNA damage and SSBs. DNA replication errors resulting in deoxyribonucleotide 5'-triphosphate (dNTP) misincorporation, depurination, deamination, base mismatches and insertions/deletions are also a potential threat to the genome [1].

Mitochondrial DNA (mtDNA) is particularly prone to oxidative DNA damage for a variety of reasons, including its close proximity to the electron transport chain where the majority of ROS is generated and the fact that it is not protected by histones [3]. The importance and consequences of mitochondrial DNA mutations was for a long time not 
given as much attention as the nuclear genome but with improvements in the ease of sequencing the mitochondrial genome, the key role mitochondrial mutations play in ageing, age-related diseases, neurodegenerative diseases and cancer has become apparent $[4,5]$.

The main repair pathways involved in the repair of DNA damage are: base excision repair (BER), double strand break repair (homologous recombination- (HR) and nonhomologous end joining (NHEJ)), nucleotide excision repair (NER), mismatch repair (MMR) and direct reversal (Figure 1.1). The focus of this study is on the MMR system and the repair of oxidative DNA damage especially in the repair of mitochondrial DNA $(\mathrm{mtDNA})$ damage. 


\section{Figure 1.1}

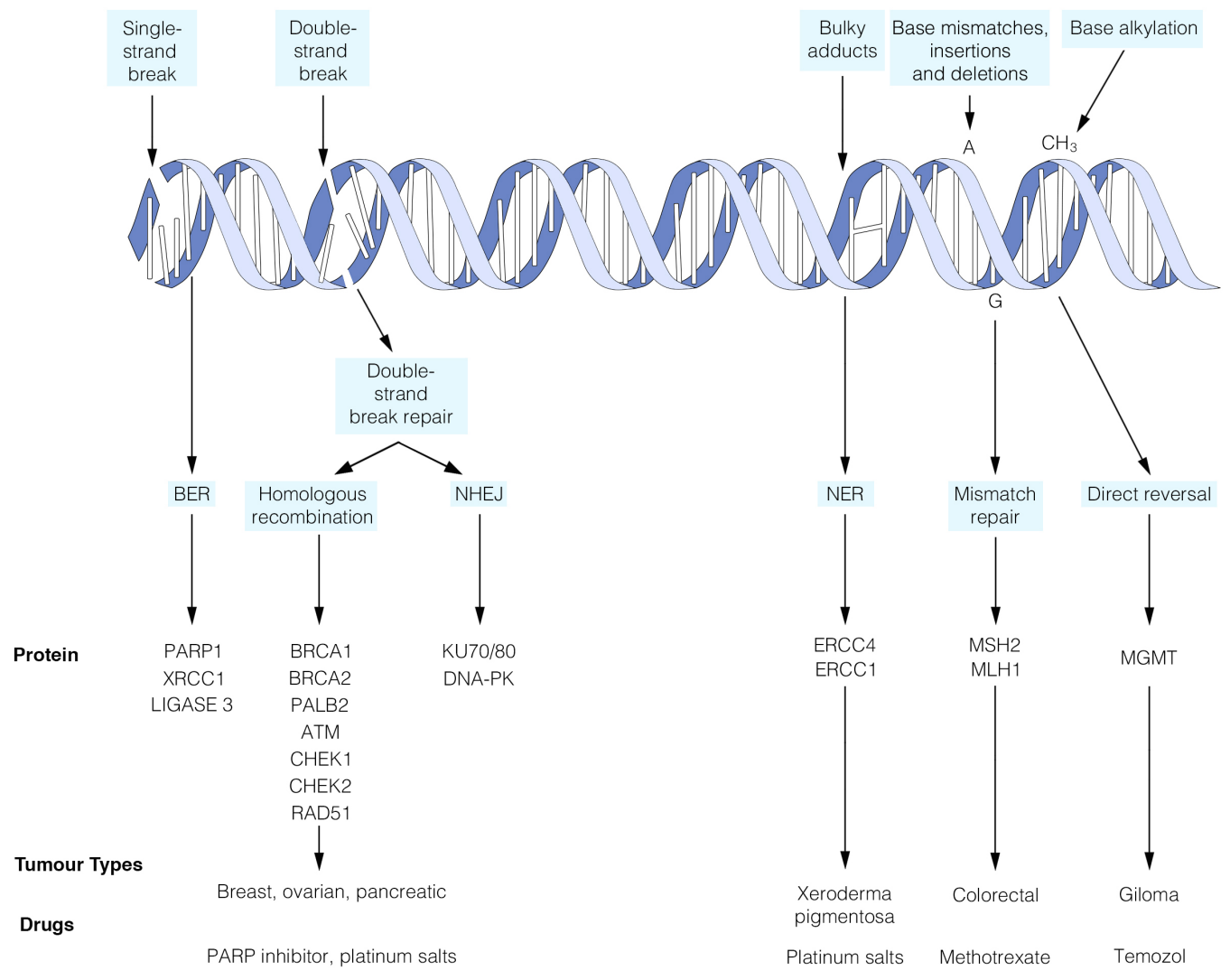

Figure 1.1 The DNA repair pathway and DDR proteins recruited depends on the type of DNA lesions present.

DNA is continually exposed to a range of insults leading to a variety of lesions. The choice of repair mechanism is determined by the type of lesion. Key DNA repair pathways, associated DDR proteins involved in each of these pathways, the tumour types commonly affected by defects in these DNA repair pathways and the drugs that have been shown to target these defects are illustrated. BER (base excision repair), NER (nucleotide excision repair), NHEJ (nonhomologous end-joining). Adapted from Lord, C et al. (2012) Nature [1]. 


\subsection{The mismatch repair (MMR) pathway}

\subsubsection{The canonical role of the MMR pathway}

The main function of the MMR pathway is the repair of post replicative DNA errors, specifically base-base mismatches and insertion/deletion loops (IDLs). A schematic of this pathway is illustrated in Figure 1.2. The MutS and MutL family of proteins are highly conserved from lower organisms to eukaryotes and are the key players in the functioning of the MMR system [6]. The majority of evidence implicates MutSa (comprised of $\mathrm{MSH} 2$ and $\mathrm{MSH} 6$ ) in the recognition of single base-base mismatches and small IDLs of 1-2 mispairs. MutS $\beta$ (comprised of MSH2 and MSH3) is involved in the recognition of longer IDLs up to 16 nucleotides long [7, 8]. Furthermore, it is well established that the degree of mismatch recognition by the MutS Complex Is dependent on the actual mismatch with high affinity being demonstrated for the common mismatch G-T and to IDL's with a single unpaired nucleotide compared with the less common C-C mispair which is not recognized by the MMR pathway $[9,10]$.

There are several heterodimers of MutL homologues including MutL $\alpha$ (complex of MLH1 and PMS2), MutL $\beta$ (complex of MLH1 and PMS1) and MutLY (complex of MLH1 and MLH3). It is widely accepted that MutL homologues are able to bind to DNA and also hydrolyze ATP [11, 12]. The role of MutLa is the best described of all the MutL homologues. The evidence suggests that MutLa is recruited by a mismatch bound MutS and this signals the presence of the error, thereby allowing the recruitment of the downstream proteins necessary to complete repair $[6,13]$. However, data about the exact nature of these protein interactions is limited. Plotz et al [14] have demonstrated that the association of MutS $\alpha$ and MutL $\alpha$ on DNA requires ATP and occurs through the $\mathrm{N}$-terminal region of MLH1. The investigators also showed that MutS $\beta$ was able to bind to MutLa as effectively as MutSa and also through MLH1 [11]. MutLy is thought to be involved in repairing some IDLs as well as having a role in meiotic recombination and no definitive role for MutL $\beta$ in the MMR pathway has been elucidated $[6,15,16]$.

The MMR protein complexes are ATPases and possess a Walker ATP-binding motif through which they are able to bind to ATP/ADP and carry out their mechanism of action [17]. For the MMR system to repair DNA replication errors that have occurred in daughter DNA, it is imperative that the system recognizes and differentiates between 
template DNA and newly synthesized DNA. The exact mechanism which allows this differentiation is not clear but the current understanding is that gaps between okazaki fragments may serve as the discrimination signal on the lagging strand but no corresponding signal on the leading strand had been established until recently $[18,19]$. The long unanswered question about the discrimination signal has potentially been addressed by a recent study by Pluciennik et al [20]. The authors of this study have shown that MutL $\alpha$ associates with a proliferating cell nuclear antigen (PCNA) molecule and incorporates additional nicks in the discontinuous strand allowing MMR to identify the nascent strand in both leading and lagging strands [20]. There are also several groups who have identified a possible additional mechanism, which may serve to make the process of identifying the nascent strand more efficient. This system is dependent on the RNases $\mathrm{H} 1$ and $\mathrm{H} 2$, which remove ribonucleotides from DNA leaving additional nicks which can be used as entry sites for EXO1[21].

Current evidence suggests that PCNA is likely to recruit components of the MMR system to replicating DNA [22] and may even have a role in assisting MutS in detecting mismatches within large regions of DNA [23]. PCNA has been shown to be able to associate with MutSa, MutS $\beta$, MutLa.

There are several proposed mechanisms of how the MMR system operates, which fall under the two main headings of "moving models" and "stationary models"[6]. Following the recognition of a mismatch by MutSa and in turn the recruitment of MutLa, the moving models essentially result in the MutS/MutL complexes leaving the mismatch they have encountered, made possible by the fact that these MMR complexes possess ATPase activity [24]. The resulting moving clamps diffuse along the DNA in a uni ("translocation model") or bi-directional manner ("sliding clamp model") from the mismatch [24, 25]. Repair of these lesions takes place when one of these clamps comes across a strand break 5' from the mismatch where replication factor C (RFC) is present and is displaced, to allow exonuclease 1 (EXO1) to access the daughter strand DNA to initiate strand degradation in the 5'-3' direction. The role of RFC is to load PCNA [26] which is essential for 3'-directed excision and inhibit EXO1 from carrying out hydrolysis in the 5' to 3' direction when the nick is 3 ' to a mismatch [27]. Given that EXO1 can only function 5' to 3' it requires the EXO1 endonuclease activity of MutLa in order to carry out 3 ' excision. The endonuclease activity of MutLa is activated by PCNA 
associating with MutL $\alpha$ and in turn causing additional nicks in the discontinuous strand to be used as EXO1 loading sites [20]. Replication protein A (RPA) is responsible for stabilizing the parental strand [28] and once the mismatch is removed EXO1 ceases to be activated by MutS $\alpha$ and is possibly suppressed by MutL $\alpha$ [29, 30]

The resulting gap is filled by the DNA polymerase, pol $\delta$, and PCNA has also recently been implicated in this re-synthesis part of the pathway since it is a processivity factor for DNA polymerase $\delta$ and $\varepsilon$ [31]. Ultimately DNA ligase I (LIG1) closes the gap in the strand to complete the repair process $[6,15]$.

The "stationary" model, which is also termed the "DNA bending/verification" model, proposes that the MMR complexes remain at the mismatch allowing the DNA to bend or loop, thereby permitting contact between the mismatch and the strand discrimination signal.[29] A schematic of the mismatch repair pathway is shown in Figure 1.2. 
Figure 1.2

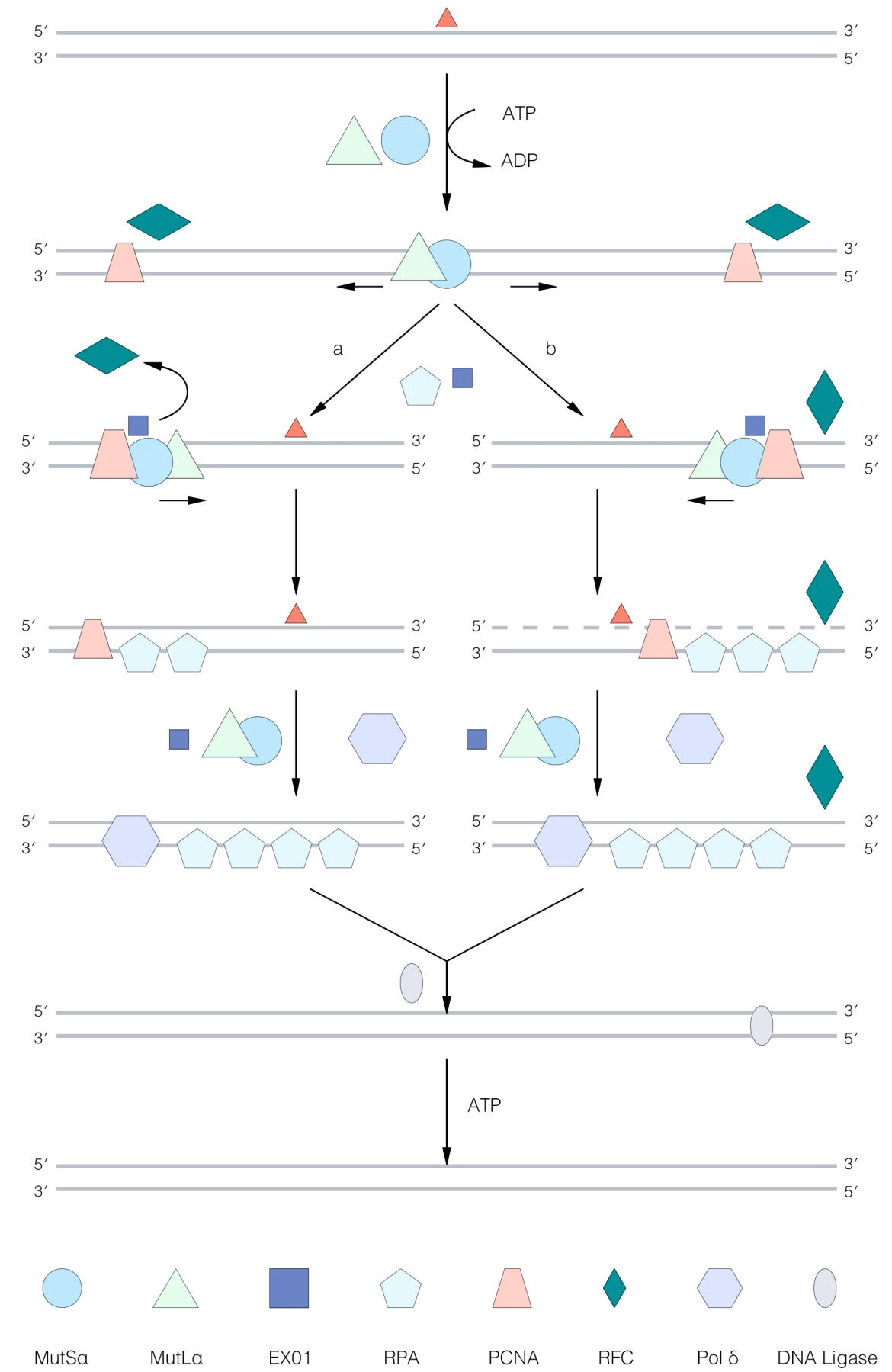




\section{Figure 1.2. Schematic of the MMR system.}

The presence of a mismatch (red triangle) results in binding of MutS $\alpha$ or Muts $\beta$ to the lesion and in turn recruitment of MutL $\alpha$. The ATPase activity of the MMR proteins allows a conformational change of the MutS/MutL complex and in turn release of the sliding clamp from the mismatch site (sliding clamp model). (A) The clamp encounters a strand break $5^{\prime}$ from the mismatch and RFC is present here and is displaced to allow EXOI access. EXOI is activated and carries out strand degradation in a $5^{\prime}$ to $3^{\prime}$ direction. RPA stabilizes the single-stranded gap. When the mismatch is removed, EXO1 activity is no longer stimulated by MutS, and is actively inhibited by MutL $\alpha$. Pol loads at the 3 ' terminus of the original discontinuity, which carries a bound PCNA molecule. This complex fills the gap and DNA ligase I seals the remaining nick to complete the repair process. (B) Clamps that migrate downstream encounter a PCNA molecule that is bound at the $3^{\prime}$ terminus of the strand break. The endonuclease activity of MutL $\alpha$ is activated by its association with PCNA and results in additional nicks in the discontinuous strand to be used as EXO1 loading sites that allows $3^{\prime}$ to $5^{\prime}$ directed excision by EXOI. The recruitment and the activation of EXO1 results in the degradation of the region between the original discontinuity and the mismatch. RFC that is bound at the $5^{\prime}$ terminus of the discontinuity prevents degradation in the $5^{\prime}$ to $3^{\prime}$ direction (away from the mismatch). Once the mismatch is removed and the EXO1 activity is inhibited by bound RPA and MutL $\alpha$, the gap is filled by the polymerase. DNA ligase I seals the remaining nick to complete the repair process. Adapted from Jiricny, J et al. (2006) Nat Rev Molecular Cell Biology [13]. 


\subsubsection{The role of MLH1 in the MMR pathway}

MutLa was first discovered in 1995 as a consequence of a study by $\mathrm{Li}$ et al, which identified the hMLH1 and hPMS2 heterodimer [32]. The authors were able to restore MMR activity to nuclear extracts of the MMR deficient $\mathrm{H} 6$ colorectal cancer cell line by the addition of what is today known as MutL $\alpha$ from HeLa cells [32]. It was later ascertained by several authors that this heterodimer is recruited to heteroduplex DNA after MutS has recognized a mismatch and that this occurs in an ATP dependent fashion [32-34]. Studies in both yeast and mammalian cell lines have shown that the MLH1 protein is essential for functional MMR $[35,36]$. The importance of MLH1 in the MMR pathway is evident from the fact that studies involving large databases (International Collaborative Group on Hereditary Nonpolyposis Colorectal Cancer (ICGHNPCC) and current International Society for Gastrointestinal Hereditary Tumors (InSiGHT)) of patients with the heritable disorder HNPCC have shown that almost half of these patients have germline mutations associated with MLH1 loss [37]. Furthermore, it was shown amongst these patients there were in the region of 500 downstream mutations as a result of MLH1 loss. Hundreds of possible mutations in the MLH1 gene including missense mutations and have been identified in patients with HNPCC leading to a range of studies aiming to identify the functional significance of these mutations $[38,39]$. In addition to the loss of MLH1 through germline mutations, the majority of MMR deficient (dMMR) sporadic tumours are also due to MLH1 loss mainly due to promoter hypermethylation of $\mathrm{CpG}$ islands in DNA [37]. The phenotype associated with promoter hypermethylation is known as $\mathrm{CpG}$ island methylator phenotype (CIMP) and has been found to be strongly linked to BRAF V600E mutations as well as older age and female sex [40, 41].

The MutL homologues are known to part of the GHKL superfamily of ATPases [42]. MutLa has been shown to carry out the key role of recruiting proteins necessary for MMR by direct interaction through ATP binding and hydrolysis causing conformational changes. [43, 44]. In both eukaryotes and prokaryotes it has been established that this occurs through ATP dependent conformational change in the $\mathrm{N}$-terminus and resulting alterations to the orientation of this terminus relative to the $C$ terminus [11]. . Plotz et al have shown that the interaction between DNA bound MutSa and MutLa takes place on the beta-sheet of the MLH1 ATP binding pocket [11]. Furthermore, they demonstrated 
that some MLH1 mutations can stop the interaction between MutS and MutL as well as abolish MMR activity [39]

It was widely believed amongst investigators in the MMR field of study that MutLa had unexplored functions, which needed to be elucidated given the huge clinical significance of MLH1 loss. Kadyrov et al explored the role of MutLa in 2006 and were able to show that human MutLa has endonuclease activity that is essential for 3'-directed MMR [45] . The activity of MutLa was found to be RFC, PCNA, ATP and Mutsa dependent and biased to nicked hetroduplex DNA. Activated MutL $\alpha$ has been established to introduce additional nicks in heteroduplex DNA 5' to the mismatch. This allows EXO1 to carry out 5 ' to 3ry out 5tc. This finding that MutLa has endonuclease activity and can provide a 5' terminus cleared the unanswered question of how Exo1, which is known to hydrolyze nicked DNA in the 5' to 3' direction is able to carry out 3' to 5' activity. The endonuclease active site is not fully ascertained but is thought to be on PMS2 [45]. Furthermore, the clinical relevance of the endonuclease activity of MutL $\alpha$ is displayed by the fact that mutations in yeast that impair this activity result in genome instability and display a mutator phenotype [46]. A later study in 2009 aimed to re-examine the role of MutLa and RPA since there were conflicting studies present describing their function [30]. This study confirmed that whilst RPA was essential in terminating excision by MutS $\alpha$-activated EXO1, MutL $\alpha$ does not participate in this process [30]. The most recent study aiming to further clarify the role of MutL was published in 2015 and used single cell fluorescence resonance energy transfer (FRET) to establish the nature of its interaction with MutS in Thermus aquaticus [47]. Qiu et al showed that MutL $\alpha$ actually has a more fundamental role than previously believed. MutL $\alpha$ in Thermus aquaticus was shown to trap MutS at a DNA mismatch before it formed a sliding clamp. This led to a structure of dynamic proteins at a mismatch, which was found to contain more MutL than MutS. This structure was found to be the area where PCNA could interact with MutL and MutS, and allowed for a more efficient mismatch repair system since MutL could carry out its endonuclease activity at the point of the mismatch specifically [45, 47]. 


\subsection{Non Canonical roles of the MMR system}

Although the main role of the MMR pathway is the repair of DNA replication errors, there is evidence that it has several other non-canonical roles including the repair of oxidative DNA damage, repair of DNA double strand breaks, participating in homologous recombination, mitotic and meotic recombination, immunoglobin class switching and co-activation of oestrogen receptor alpha $[12,15]$.

\subsubsection{Meiotic and mitotic recombination and MMR}

The role of the MMR proteins in both mitotic and meiotic recombination has been confirmed in numerous in vitro and in vivo studies [48-52]. The function of MMR during recombination is believed to be due to the interaction of MMR factors with mismatches on heteroduplex DNA $[53,54]$ or possibly with other structures such as Holliday junctions [55].

\subsubsection{MMR and meiotic recombination}

The MMR proteins MLH1 and MLH3 have been shown to function with a MutS homologue, hMSH4/hMSH5, which has no known role in the conventional MMR pathway to carry out their role in meotic recombination. The double holiday junction is an intermediate in meiotic recombination [56] and the role of meiotic MMR is to process DSBs through this pathway resulting in genetic recombination and accurate segregation of homologs [51]. MSH4 and MSH5 have been found to be expressed in the testes and ovaries during early prophase I of meiosis and have been found to have a role in recombination and crossover events [56]. MSH4/MSH5 has been shown to bind to recombination intermediates and cause exchange of ADP with ATP leading to a conformational change resulting in a hydrolysis-independent sliding clamp, which dissociated from the holiday junction. This was proposed to stabilize the recombination intermediate until it was positioned to allow accurate parental chromosome segregation [52]. MLH3 is essential for the binding of MLH1 to meiotic chromosomes in early pachynema of prophase this heterodimer and I associates with MSH4/MSH5 to allow chromosomes to segregate accurately during the first meiotic division [57]. 


\subsubsection{MMR and mitotic recombination}

The MMR pathway has been found to have anti-recombinogenic activity and MMR deficiency has been shown to result in increased frequency of recombination between homeologous sequences including gene conversions and/or crossover [48-50]. The MMR proteins have a role in mitotic recombination by preventing strand exchange and recombination between divergent sequences. The exact mechanism by which the MMR system prevents strand exchange is unclear but is thought to be by blocking of the formation of heteroduplex DNA, whenever well-recognized mismatches are formed by strand exchange between non-identical parental sequences [58]. Genetic studies of homeologous recombination during mitosis in yeast suggest that there are two stages of regulation of recombination between divergent sequences. The first stage is that if recipient and donor sequences are too divergent $(>10 \%)$, recombination is severely repressed likely due to an inability to form a sufficiently stable base-paired intermediate. At lower levels of divergence, MMR imposes an additional barrier to recombination so that it suppresses the formation of heteroduplex DNA in proportion to the increase in sequence diversion [59]. Analysis in S. cerevisae examining the requirement for different MMR complexes in repression of homeologous recombination and found that a mutation in the $\mathrm{MSH} 2$ gene results in the highest rate of recombination followed by MSH6 and MSH3. The MutL homologs (MLH1 and PMS2) resulted in a much lower homologous recombination level [50]. Furthermore, Nicholson et al have identified that different MMR proteins are required for the recognition of differing mismatches in mitotic recombination intermediates. For example, $\mathrm{MSH} 2$ was required for the recognition of all mismatches but MSH6 only recognized base-base mismatches and IDLs that were one base pair long [60]. MSH2 deficient mice have been found to have an increased rate of haematological maliganancies thought to be due to chromosomal rearrangements [61].

\subsubsection{MMR and immunoglobulin diversification}

To combat the huge variety of antigens that we are exposed to our immune system is required to constantly produce a range of antibodies. This diversity is accomplished by several mutagenic processes that take place at the immunoglobulin locus. This is a two stage process, the first of which occurs early in B cell development and the second after exposure of a B-cell to an antigen [62]. The second stage involves the enzyme 
activation-induced deaminase (AID) which converts cytosine to uracil in single stranded DNA and initiates mutagenic processes such as $U: G$ mismatches resulting in the recruitment of MutS and MutL[63]. The recruitment of the MMR proteins leads to activation of the exonuclease Exol and paradoxically leads to error-prone repair involving the translesional synthesis (TLS) DNA polymerase $h$ that promotes A:T mutagenesis and DSBs. This results in enhanced mutation frequency of AID activity as well as broadening the spectrum of base mutations and efficiency of antibody maturation [64]. Studies have shown that MSH2, MSH6 and Exol-deficient B cells have a significantly lower (80-90\% less) rate of A:T mutations $[64,65]$. No role for MSH3 has been found in immunoglobulin diversification and studies suggest that MutSa is involved in this role of the MMR pathway [64]. MutSa has been found to interact with $U: G$ mismatches as well as initiate its catalytic activity [66].

\subsubsection{MMR and chromatin assembly}

MMR activity occurs in the chromatin environment, which led investigators to assess whether the MMR proteins have any role in chromatin assembly. Schopf et al and others have shown that presence of a mismatch in nicked plasmid heteroduplex DNA delays nucleosome loading in human cell extracts and once the mismatch is removed, repair of the single strand gap and nucleosome loading occurs [67, 68]. The investigators also illustrated that the chromatin assembly factor CAF-1 interacts with MSH6. Furthermore, PCNA was also found to interact with both CAF-1 and MutSa and in vivo this interaction was found to increase during $S$ phase and was likely dependent on CAF-1 phosphorylation [68]. The authors proposed an outline of the function of MMR in chromatin assembly on mismatch containing DNA based on the study they performed: 1, MutSa sliding clamps may physically interfere with nucleosomes 2, MutSa may stop CAF-1 interacting with PCNA (dependent on CAF-1 phosphorylation status or physical interference) and once repair is completed and MutSa is no longer present, CAF-1 can interact with PCNA and resume chromatin assembly [68]. The histone mark H3K36me3 can interact with MSH6 to chromatin in S phase prior to DNA replication [68] and in yeast the acetylated and deacetylated forms of the histone $\mathrm{H} 3$ K56 has been shown to act synergistically with the MMR proteins to reduce mutagenesis [69]. 


\subsection{MMR activity in the mitochondria}

Mitochondrial DNA is particularly prone to oxidative DNA damage for a variety of reasons, including its close proximity to the electron transport chain where the majority of ROS is generated and the fact that it is not protected by histones [3]. It is estimated that the levels of oxidative damage in the mitochondria are 2-3 times higher than in nuclear DNA [70, 71]. It has been established that mitochondria utilise BER as their primary mechanism for repairing mitochondrial oxidative DNA damage [72]. Nevertheless, there is increasing evidence that some form of MMR machinery is present in the mitochondria and that MMR proteins are potentially also involved in the repair of oxidative DNA damage to mtDNA [73-75].

One of the earliest studies to illustrate that MMR activity may be present in the repair of mtDNA was carried out by Habano et al. who examined nine microsatellite sequences in the mtDNA of 45 sporadic CRCs [76]. The investigators found that in $44 \%$ of these cancers there was an alteration in a polycytidine $(C) n$ tract within the non-coding displacement-loop (D-loop) region and that three of the samples exhibited frameshift mutations within microsatellite tracts in NADH dehydrogenase genes (Complex I $(\mathrm{Cl})$ ) [76]. Since MSI is strongly associated with MMR deficiency, the authors concluded that given $\mathrm{MSI}$ is present in mtDNA, that some form of MMR activity is likely to be taking place in the mitochondria. In addition, a mitochondrial MutS homolog (msh1) that detects mismatches has been identified in Saccharomyces cerevisiae [77]. Several studies have tried to definitively show the proteins and steps involved in a proposed mammalian mitochondrial MMR system but to date no clear pathway has been fully elucidated. Mason et al showed mismatch repair activity in rat liver mitochondrial lysates by using a nicked heteroduplex substrate in the $\beta$-galactosidase reporter gene (with a GT or GG mismatch) as a model and concluded that the MMR activity was bidirectional, ATP dependent and EDTA sensitive [74]. Hashiguchi et al. discovered that the mitochondrial extracts from three different human cell lines were able to bind mismatches and small insertion-deletion loops [73]. The authors hypothesised, using polyacrylamide gel migration, that the protein involved is likely to be $80-90 \mathrm{kD}$ and may function alone. The protein was able to bind 8-oxoG/G/A/T suggesting that it may be involved in repairing oxidative damage in the mitochondria [73]. 
two studies have demonstrated that $\mathrm{MSH} 2$ is unlikely to be involved in mitochondrial MMR since mitochondrial extracts from MSH2 deficient cells retained mismatch repair activity $[73,75]$. DeSouza et al are the first investigators to propose a novel protein that potentially functions as a player in MMR [75]. They were unable to detect mitochondrial localization of the nuclear MMR proteins, MLH1, MSH3 and MSH6 in the mitochondria of human HeLa cells by immunofluorescence but they identified that silencing the $Y$ BOX binding protein (YB1) resulted in reduced MMR activity in mitochondrial extracts [75].

A very recent study in retinal endothelial cells aimed to establish the role of the MMR proteins in diabetic retinopathy given that this hyperglycaemia related complication is known to cause mitochondrial dysfunction, ROS induction, increased oxidative damage in the mitochondria and decreased levels of POLG [78]. The authors showed that expression of MLH1 and MSH2 was significantly decreased in retinal endothelial cells exposed to high glucose compared to normal glucose. The expression levels of MSH6, YB1 and Pms2 were maintained in high glucose conditions. The deficiency in the MMR proteins was associated with an increased number of mismatches (increased sequence variants) in the non-coding D-loop in the mtDNA in association with decreased respiration and increased apoptosis. This phenotype was rescued by the overexpression of MLH1 but not with the overexpression of MSH2 [78] .

As mentioned in the section on synthetic lethal approaches (section 1.3), we have recently shown further evidence of a potential role for MMR in the mitochondria [79]. No role for the conventional MMR proteins has to date been implicated in the mitochondria so this study was the first to identify a specific MMR protein, MLH1, to be involved in the repair of oxidative DNA lesions in the mitochondria.

\subsection{MMR deficiency and cancer}

Deficiency of one or more of the MMR proteins has been reported to be present in various tumour types including $15-17 \%$ of all primary colorectal cancers [80, 81], around $30 \%$ of endometrial cancers [82] and approximately $2-29 \%$ of ovarian cancers [83]. 
These proteins have been found to be absent due to epigenetic silencing, inherited germline mutations and somatic mutations. Loss of MLH1 frequently due to promoter hypermethylation and $\mathrm{MSH} 2$ loss have been found to be the most common cause of sporadic mismatch repair deficient colorectal cancers [84, 85]. Studies have established that promoter hypermethylation is commonly bi-alleic and that the mechanism behind this process is still not clear but it has been found to be present in early colorectal adenomas [37]. Lynch syndrome is a heritable disorder, which predisposes affected individuals to several cancers such as colorectal, endometrial, ovarian, gastric, urothelial and small bowel [86]. This autosomal dominant disorder is caused by a germline mutation in one copy of an MMR gene, followed by a 'second hit' to the remaining normal allele, which results in complete gene loss. It has been estimated that Lynch syndrome accounts for $3-5 \%$ of CRC cases and in $70 \%-90 \%$ of these cases, the causative mutation is in the MLH1 or MSH2 gene [87].

It is well established that mutation rates are significantly higher in human tumour cells with MMR deficiency compared to wild type cells, resulting in an accumulation of unrepaired mutations throughout the genome, commonly known as the 'mutator phenotype' [88]. Therefore MMR deficient tumour cells possess mutation rates that are 100-1000-fold greater than that of normal cells [88, 89]. These mutations are normally frameshift mutations that arise during replication and result in truncated proteins. Due to their repetitive nature, the mutator phenotype has been found to result in mutations within microsatellite sequences (microsatellite instability (MSI)). MSI due to loss of MMR often occurs in genes that have an impact on tumour development and biology such as those involved in signal transduction (TGFBR2, PTEN, BRAF), apoptosis (BAX, Caspase-5), DNA repair (MRE11A, RAD50) and damage signalling (ATR, CHK1) [85, 90]. The loss-of-function mutations in tumour suppressor genes and gain of function mutations in oncogenes associated with MMR deficiency are thought to not only drive the oncogenic process but are likely to also have an impact on tumour biology and treatment response [85].

In clinical practice MMR deficiency and the frequently associated $\mathrm{MSI}(>90 \%)$ are diagnosed by DNA sequencing, PCR to detect changes in the length of microsatellites and immunohistochemistry staining for MMR proteins. Specific features have been associated with MMR-deficient tumours are described in Table 1.1 [86, 91]. The revised Bethesda guidelines were established as a tool to identify those patients that would 
warrant testing for MSI with an emphasis on diagnosing patients with Lynch syndrome who's management would be affected by this diagnosis [92] (Table 1.2). The PCR method to detect MSI involves using a fluorescent multiplex PCR assay to examine five primary microsatellite loci; Bat-25, Bat-26, D2S123, D5S346, and D17S250 which were established at the 1997 National Cancer Institute-sponsored conference on MSI [93].This method has been validated and is now internationally standardized with a defined panel of microsatellite markers and scoring system (Table 1.3) [85, 94]. It is important to note that that $\mathrm{MSI}$ has been validated to be a good marker for MMRdeficient associated with MLH1, MSH2 and PMS2 but the vast majority of MSH6 deficient tumours do not display MSI [95]. 


\section{Table 1.1}

- Proximal

- Poorly differentiated

- Histology-mucinous (15\%), signet cell and medullary subtypes

- Presence of tumor infiltrating lymphocytes, Crohn's-like lymphocytic reaction

- Enhances tumour formation from tubulovillous adenoma to carcinoma within 2-3 years in Lynch syndrome

Table 1.1. Features of tumours with dMMR $[86,91]$.

Table 1.2

- Colorectal cancer diagnosed in a patient who $<50$ years of age.

- Presence of synchronous, metachronous colorectal, or other HNPCC-associated tumours," regardless of age.

- Colorectal cancer with the MSI-H histology (Table 1.3) diagnosed in a patient who is $<60$ years of age.

- Colorectal cancer diagnosed in one or more first-degree relatives with an HNPCC-related tumour, with one of the cancers being diagnosed $<50$ years.

- Colorectal cancer diagnosed in two or more first- or seconddegree relatives with HNPCC-related tumours, regardless of age.

Table 1.2. Revised Bethesda Criteria for identifying individuals at high risk of MMR deficiency [92].

* These tumours include colorectal, endometrial, stomach, ovarian, pancreas, ureter, renal pelvis, brain tumours, sebaceous gland adenomas, small bowel and keratocanthomas 


\section{Table 1.3}

\begin{tabular}{|l|l|}
\hline MSI-Low & $1 / 5$ markers positive \\
\hline Microsatellite stable (MSS) & $0 / 5$ markers positive \\
\hline MSI-High & $\geq 2$ markers positive \\
\hline
\end{tabular}

Table 1.3. Bethesda scoring system for the diagnosis of MSI [92].

\subsubsection{Treatment of MMR cancers}

MMR deficiency is the cause of a significant number of colorectal cancers $\left(4^{\text {th }}\right.$ most common cancer in the UK, CRUK 2013) and endometrial cancers ( $4^{\text {th }}$ most common cause of cancer in women, CRUK 2013). Given the involvement of mismatch repair deficiency in some of the most common cancers, it is important to understand the biology of these cancers and the response to current treatments available

Most in vitro and clinical studies have shown MMR deficient cancers to be resistant to standard chemotherapeutics including 5-FU [96], Cisplatin, Carboplatin and possibly topoisomerase inhibitors [85]. Overall is seems that patients with dMMR tumours have an improved overall survival when compared with MSS CRCs of the same stage including patients with metastatic disease [97]. Nevertheless, given the high incidence of colorectal cancer worldwide and the problems with drug resistance with dMMR tumours, there is still a huge need to identify the most effective way of treating this group of patients. The MMR pathway is essential in the efficacy of many drugs due to the fact that it has a role in recognizing many drug-induced DNA adducts and coordinating the downstream DDR through ataxia-telangiectasia mutated (ATM) and ataxia telangiectasia and Rad3-related protein (ATR) [98, 99]. MutS has been shown to bind MutL in the presence of a drug-induced DNA adduct that cannot be repaired and then subsequently recruiting ATM/ATR leading to cycle cycle arrest (to allow repair) or apoptosis [6]. The two main models that are widely believed to be the method in which the MMR pathway recruits ATM/ATR are the futile cycling model and the direct signaling model. The direct signaling model proposes that a drug-induced lesion would normally cause MutS and MutL to directly recruit ATM/ATR and cause cell cycle arrest and apoptosis instead of mediating repair. The futile signaling model states that a 
mismatch would be recognized and removed from the newly synthesized strand by the MMR system leaving behind a strand that contains 5FdUTP. This would allow the synthesis of DNA with mismatches and subsequently set up a futile cycle of mismatches causing persistent strand breaks, stalled replication forks, recruitment of ATM/ATR and cell cycle arrest and apoptosis [100]. In a dMMR cell, the drug induced lesions would not be recognized to cause cell death and subsequently this leads to drug resistance.

\subsubsection{5-FU and dMMR}

The antimetabolite 5-FU is the most effective and commonly used drug for the treatment of CRC [101-103]. The current available evidence has led to adjuvant 5-FU based chemotherapy (usually in combination with Oxaliplatin) being recommended as standard of care for patients with stage III CRC [103, 104]. For patients with stage II $\mathrm{CRC}$ the benefit of adjuvant chemotherapy has been found to be small and therefore it is generally only recommended in patients with high risk features [104, 105]. The efficacy of 5-FU in dMMR tumours has not been definitively confirmed since several retrospective trials have produced differing results as described below [106-108].

A study by Meyers et al. found that the MLH1-deficient CRC cell line HCT116 is 18 times less responsive to 5-FU treatment compared to its isogeneticallymatched pair HCT116+chr3 in long-term clonogenic assays [100]. Upon further analysis of the mechanism behind this observation, the authors showed that there were a greater number of MMR proficient cells in the G2/arrest phase of the cell cycle which would eventually lead to cell death and apoptosis compared to the MMR deficient cell lines [109]

From a clinical perspective, the management guidelines in most centers, based on the current available evidence is that 5-FU based chemotherapy should be recommended to patients with MSI tumours only in stage III CRC and not in stage II CRC [104] .Several studies have shown no benefit for treating patients with MSI tumours with 5-FU based adjuvant treatment. Sargent et al (2010) carried out a large study examining a pooled data set of 1027 patients ( $n=165$ with dMMR) with both stage II and stage III tumours [107]. Fifteen percent of the 457 patients that were part of one study had 
dMMR and exhibited an improved survival compared to the MSS tumours but showed no improved outcomes with adjuvant 5-FU treatment. The results of this study were validated by combining the data from an earlier study by Ribic et al consisting of 570 patients, 96 of these patients had MSI tumours [106]. This study further confirmed that MSI tumours were associated with improved outcomes but that 5-FU based adjuvant treatment abrogated this improved outcome [107]. Furthermore, this part of the study confirmed a worse OS for stage II patients with $\mathrm{MSI}$ tumours that received 5-FU treatment versus surgery alone [106]. The commentary following these studies was that the small numbers of patients with MSI tumours meant that larger studies needed to be carried out before a definitive decision regarding dMMR and adjuvant chemotherapy could be reached and the difference in treatment between stage II and III MSI tumours established [110]. The role for adjuvant 5-FU chemotherapy in stage II CRC with MSI was also studied in the QUASAR trial where these patients were either randomized to receive 5 -FU $(n=1,483)$ or surgery alone $(n=1,480)$. The improved outcome of patients with MSI tumours was again confirmed with the risk of recurrence being $11 \%$ in this group compared to $26 \%$ in the MSS tumours. There was however no benefit of adjuvant chemotherapy demonstrated in the $M S I$ group $(H R=0.97, P=0.92)[111]$

A more recent study in 2011 by Sinicrope et al was a large study involving 2141 stage II and III patients with CRC by including patients treated in several randomised studies of 5-FU based adjuvant chemotherapy. In this study population, 344 patients $(16.1 \%)$ had MSI tumours (164 stage II and 180 stage III tumours) and had an improved outcome in the treated and untreated group compared to patients with MSS tumours [108]. The MSI cohort (treated and untreated) had a reduced 5-year recurrence rate of $22 \%$ vs $33 \%$ $(p=0.001)$, delayed time to recurrence (TTR) $(p=0.001)$ and improved OS $(p=0.004)$ [112]. This study showed a statistically significant increase in DFS ( $p=0.47)$, TTR $(p=0.016)$ and OS $(p=0.041)$ in both stage II and stage III MSI CRC compared to MSS tumours but upon carrying out univariate analysis in the MSI group treated with 5-FU based chemotherapy vs surgery alone or no 5-FU, the improved outcome was only significant for the stage III patients. In the patients with stage III cancers and dMMR, treatment with 5-FU resulted in a reduced distant recurrence rate of $11 \%$ vs $29 \%$; ( $\mathrm{P}=$ 0.11). This result confirmed that 5-FU treatment in patients with MSI does not abrogate the known improved survival in this group of patients. It is important to note that the outcome of patients with MSI tumours treated with 5-FU was not compared to the 
untreated patients with MSS tumours. Interestingly, Sinicrope et al examined the difference in benefit from 5-FU between sporadic and germline (Lynch Syndrome) dMMR tumours and found that the benefit of treatment in the stage III MSI group is restricted to the tumours with germline mutations with improved DFS after 5-FU-based treatment compared with sporadic tumors where no benefit was observed $(P=.006)$ [108]. Unfortunately the investigators did not examine the treatment effect in the stage II MSI population in detail so no information regarding differences between germline and sporadic tumours in this setting is available. The criticism of this study is that despite the large study population, a stratified analysis by treatment group or stage was not performed therefore the unanswered questions from previous studies by Ribic et al [106] and Sargent et al [107] could not be answered. The control arm of the PETACC3 clinical study contained 600 patients with stage II and III colorectal cancer treated with 5-FU and this showed an improved 5-year DFS in patients with MSI tumours [113]

The pressing question of the value of using MMR status as a tool to predict outcome of adjuvant chemotherapy has still not been definitively confirmed. Most importantly a clear answer about whether patients with stage II CRC have a worse outcome with chemotherapy needs to be addressed. In clinical practice currently the only widely accepted use for dMMR as a prognostic marker in guiding treatment decisions is in patients with dMMR and stage II CRC where the risk of recurrence is low [73, 76]. The clinical community feel better datasets with more detailed information are necessary to carry out large studies examining the prognostic and predicative role of MSI in CRC. Furthermore, the recent study by Sinicrope et al has demonstrated that gene expression profiling, methylation studies and microRNA assessment will be necessary to elucidate clinically relevant biological subgroups [110].

The most recent study, the AEGO study (2016) further examined the role for adjuvant 5FU in dMMR CRC. This study was set up to examine the benefit of 5-FU in combination with Oxaliplatin (FOLFOX) versus 5-FU alone or no chemotherapy in CRC with MSI. This retrospective multicenter study included 433 patients with MMR deficient CRC who underwent curative resection for stage II and III disease between 2000-2011 [83]. In comparison to surgery alone there was an improved DFS with Oxaliplatin based chemotherapy $(H R=0.35)$ but not with adjuvant $5-F U$ alone $(H R=0.73)$. Further 
subgroup analysis revealed the benefit of combination chemotherapy was restricted to the patients with stage III disease $[51,83]$.

\subsubsection{Platinums and dMMR}

Several in vitro studies have confirmed that MMR deficient cancer cells are more resistant to the platinum based compounds Cisplatin and Carboplatin [114]. Aebi et al demonstrated that in vitro both $\mathrm{MLH} 1$ and $\mathrm{MSH} 2$ deficient cell lines were much less sensitive to Cisplatin than their MMR proficient matched cell lines [115]. This resistance is thought to be due to the DNA adducts produced by these agents which are normally recognized by an intact MMR system [85, 116]. Aebi et al. and several other groups have found that Cisplatin resistance is associated with loss of MMR proteins $[115,117]$. From a clinical perspective there are contradictory studies and as yet MMR status is not used as a predictor for the response to chemotherapy with platinum based compounds. Resnick et al. evaluated data from 158 patients with endometrial cancer who had received adjuvant platinum based chemotherapy and found that the overall survival was not different in the group with MMR deficient cancers compared to those with MMR proficient cancers [82]. In a sub-group analysis however, the group with MMR deficient, stage III/IV disease had a worse progression free survival $(p=0.031)$ [82]. Samimi et al ascertained a decreased expression of both MLH1 and MSH2 in paired ovarian tumour tissue after platinum based chemotherapy but did not find a correlation between MMR status and response to platinum based chemotherapy or overall survival (OS) [118]. Honecker et al examined the MMR status of 35 platinum resistant germ cell tumours (GCTs) compared to 100 controls. The authors found a correlation between dMMR (MLH1 and MSH2 loss) and the Cisplatin resistant tumours but found an improved OS in the $\mathrm{MSI}$ subgroup within the treatment resistant tumours [119]. Given the contradictory evidence regarding MMR status and response to platinum agents, larger scale prospective studies will be necessary to confirm whether there is a true relationship.

The platinum agent, Oxaliplatin has not shown the same resistance in MMR deficient cells. This is thought to be due to the fact that the Oxaliplatin adducts are not recognised by the MMR system [114]. Oxaliplatin is part of the standard drug combination used in the adjuvant setting in colorectal cancer but until recently the 
benefit of this combination in the setting of dMMR tumours had not been confirmed. The AGEO study carried out in 2016 has established the benefit of adjuvant Oxaliplatin based combination chemotherapy (FOLFOX) in stage III dMMR tumours [120].

\subsubsection{Topoisomerase inhibitors and anthracyclines in dMMR}

To date there are preclinical studies showing both benefit [121], no additional benefit [122] and resistance [123] to topoisomerase inhibitors in MMR deficient cancer cell lines [85]. In vitro studies have shown impaired response of cells lines exposed to Doxorubicin, Epirubicin and Mitoxantrone in MSH2 and MLH1-deficient cell lines as well as an impaired sensitivity to Camptothecin and Topotecan in MHL1 deficient cells [123]. Jacob et al demonstrated an increased sensitivity of MLH1-deficient colorectal cancer cell lines to the drugs Camptothecin and Etoposide compared to MLH1-proficient cell lines [124]. It is thought that potential reasons behind these contradictory studies is due to differing secondary mutations in the cell lines used including MRE11 and RAD50. The clinical studies are confounded by the fact that Irinotecan is normally given with 5FU but one randomized phase III study which compared adjuvant 5-FU+Leucovorin to 5-FU/leucovorin and Irinotecan (FOLFIRI) observed an improved 5 year disease free survival in the patients with MMR deficiency, in stage III CRC patients, who received Irinotecan based chemotherapy compared to the patients who had MMR proficient tumours [125]. A recent large prospective study $(n=1254)$ which randomized patients with stage II and III CRC to receiving either 5-FU or FOLFIRI showed a contradictory conclusion to the study by Bertagnolli et al [125]. Klingbiel et al did not observe a significant advantage to giving adjuvant treatment to the patients with stage III disease and although there was an improved survival with chemotherapy in the stage II, setting there was no benefit in adding in Irinotecan [112].

\subsubsection{Alkylating agent and dMMR}

Alkylating agents including the drugs Temozolomide, Procarbazine and N-methyl-N'nitro-N-nitrosoguanidine (MNNG) are commonly used to treat lymphomas, brain tumours and sarcomas. They cause damage by modifying the N-7 and O-6 on guanine residues and this type of damage is repaired by several DNA repair pathways including the MMR pathway [126]. Most in vitro and in vivo studies have concluded that Temozolomide resistance is mediated through an acquired mutation in the MSH6 gene 
$[127,128]$. Furthermore, MLH1-deficient cell lines have been shown to be resistant to treatment with MNNG [129].

\subsubsection{The mitochondrial genome}

The mitochondrial genome is maternally inherited and present in multiple copies within a cell and in differing numbers per cell depending on the metabolic needs of the tissue type [130,131]. Mitochondria have evolved to contain their own DNA (mtDNA), which is a small $(16.6 \mathrm{~kb})$, circular and encodes 37 genes. It has been established that on average mammalian cells contain $10^{3}-10^{4}$ copies of mtDNA which undergo replication independently of nuclear DNA [132]. The majority of these genes (13 genes) are dedicated to producing components of the oxidative phosphorylation (OXPHOS) system and the remaining genes encode 22 tRNAs and two rRNAs [133]. The other proteins required for the proper functioning of the OXPHOS system come from nuclear encoded gene products of which there are in excess of 50 genes involved [4].

\subsubsection{Heteroplasmy and homoplasmy}

Studying mitochondrial genetics and mtDNA mutations is complicated by the fact that mitochondria can contain both mutant DNA in a situation known as heteroplasmy. The process of cell division results in a random distribution of mitochondria in daughter cells leading to either homoplasmy where there is majority wild type or mutant DNA or a mixture of both leading to heteroplasmy [134]. This concept was established as a consequence of the heterogeneity observed within families with mitochondrial diseases caused by the same mtDNA mutation. Several studies have established the presence of mitochondrial mutations in human cancer but the significance of these mutations and their biological consequences are far from established and complicated by the presence of homoplasmy and heteroplasmy [132]. Park et al studied human cell lines carrying both homoplasmic and heteroplasmic mutations in the mitochondrial Complex I gene ND5 which have has previously been identified in a human CRC cell lines [5]. The authors established that there is a difference in tumour growth between the two cell lines with the heteroplasmic cell lines exhibiting enhanced growth and the homoplasmic cell line with the same mutations leading to decreased tumour growth [5]. 


\subsubsection{Oxidative phosphorylation}

The respiratory chain chain is located in the mitochondrial inner membrane and is compromised of five membrane bound enzyme complexes: Complex 1 (NADHubiquinone oxidoreductase), Complex II (succinate- quinone oxidoreductase, CII), Complex III (cytochrome bc1, CIII), Complex IV (cytochrome c oxidase, CIV), Complex $\mathrm{V}$ ( F1FO-ATP synthase, CV) as well as two electron mobile carriers coenzyme $Q$ and cytochrome $C$. The subunits of the respiratory chain are mainly encoded by nuclear DNA (approximately 70 subunits) with only 17 encoded for by mtDNA (Figure 1.5) [135]. These transmembrane complexes are able to undergo oxidation and reduction reactions because they have various oxidation-reduction centers, including quinones, flavins, iron-sulfur clusters, hemes, and copper ions. The transport of electrons between complexes takes place because of the mobile carriers coenzyme $Q$ and cytochrome $c$ [136]. The role of this system is to produce ATP by using the electrons from $\mathrm{NADH}_{2}$ and $\mathrm{FADH}_{2}$ produced during glycolysis, fatty acid oxidation and the citric acid cycle [137]. Glycolysis and the TCA cycle only yield approximately four molecules of ATP and the oxidative phosphorylation system produces the remaining 32 to 34 ATP molecules. The electrons from NADH initially enter Complex I and are then transferred to Complex III via flavin mononucleotide and the electron carrier coenzyme Q. Within Complex III the electrons are transferred from cytochtrome $b$ to cytochrome $c$ and finally to Complex IV, which ultimately transfers the electrons to molecular $\mathrm{O}_{2}$, which becomes reduced to form $\mathrm{H}_{2} \mathrm{O}$. Complex II receives electrons from $\mathrm{FADH}_{2}$ rather than $\mathrm{NADH}$ and these electrons are also finally transferred to $\mathrm{O}_{2}$ via coenzyme $\mathrm{Q}$, Complex III and Complex IV. The high energy electrons are transferred along the protein complexes within the electron transport chain in a series of redox reactions leading to the simultaneous pumping out of protons from the mitochondrial matrix and to the formation of an electro-chemical gradient [138]. Evidence suggests that the transfer of protons is either direct through protonation and de-protonation of redox intermediates (Complex III and Complex IV use this method) or indirect through conformational change of the complexes (Complex I and Complex V). It is estimated that approximately 10 protons are transported from the mitochondrial matrix to the inner membrane space per oxidised NADH molecule [139]. 


\section{Figure 1.6}

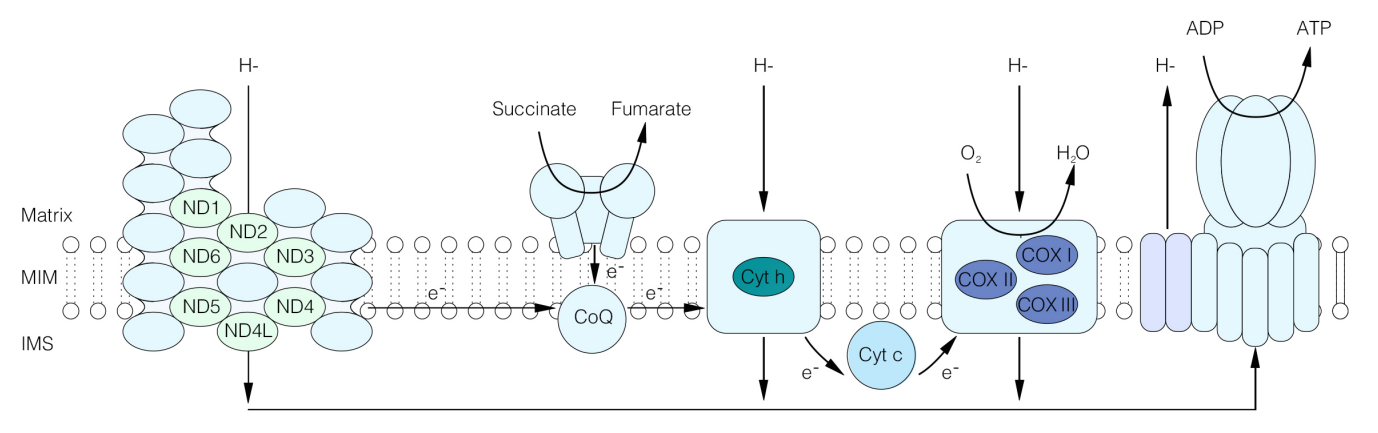

$\begin{array}{lccccc}\text { Polypetides } & \text { ComplexI } & \text { Complex II } & \text { Complex II } & \text { Complex IV } & \text { Complex V } \\ \begin{array}{l}\text { mtDNA-encoded } \\ \text { subunits }\end{array} & 7 & 0 & 1 & 3 & 2 \\ \begin{array}{l}\text { nDNA-encoded } \\ \text { subunits }\end{array} & -39 & 4 & 10 & 10 & -14 \\ \begin{array}{l}\text { Assembly } \\ \text { proteins }\end{array} & -11 & -2 & -9 & -30 & -3 \\ \end{array}$

\section{Figure 1.5. The respiratory chain/OXHPOS system}

The respiratory chain chain is composed of five membrane-bound complexes: Complex 1 (NADH-ubiquinone oxidoreductase), Complex II (succinate- quinone oxidoreductase), Complex III (cytochrome bc1), Complex IV (cytochrome c oxidase), Complex V (F1FO-ATP synthase). These OXPHOS complexes are made from both mitochondrial and nuclear DNA encoded subunits apart from Complex II that is only made from nuclear DNA encoded subunits. There are two electron carriers coenzyme $\mathrm{Q}(\mathrm{CoQ})$ and cytochrome c (Cytc). Electrons from $\mathrm{NADH}_{2}$ and $\mathrm{FADH}_{2}$ (produced during glycolysis, fatty acid oxidation and the citric acid cycle) undergo a series of redox reactions leading to the simultaneous pumping out of protons from the mitochondrial matrix across the mitochondrial inner membrane (MM) to the intermembrane space (IMS) forming an electro-chemical gradient. The final electron acceptor is molecular oxygen to produce water. The electrochemical gradient forms most of the mitochondrial transmembrane potential which allows the pumping back of protons in the opposite direction through Complex V to form ATP from ADP and free phosphate. Adapted from Schon, $E$ et al. (2012) Nat Rev Genet [4] 


\subsection{Complex I}

\subsubsection{The Structure}

The entire crystal structure of Complex I from the bacteria Thermus thermophilus at 3.3 $\AA$ resolution was recently determined providing invaluable information about the organization and function of this indispensible respiratory enzyme in humans [140]. Complex I plays a vital role in the chain by enabling the transfer of two electrons from $\mathrm{NADH}$ to ubiquinone as well as coupling this to the translocation of four protons across the inner membrane [141, 142]. Complex I is the largest of the respiratory complexes and is made from at least 45 subunits of which 14 have been found to be 'core' subunits that are highly conserved from bacteria to humans strongly suggesting that the mechanism is also conserved [143-146]. These subunits are mainly coded for by nuclear DNA [147] and imported into the mitochondria [148] but there are seven mitochondrial encoded subunits [149].

Bacterial and eukaryotic Complex $\mathrm{I}$ is an $\mathrm{L}$ shaped structure located within the mitochondrial inner membrane composed of a long hydrophobic transmembrane domain and a hydrophilic domain for the peripheral arm which protrudes into the mitochondrial matrix [150]. The structure of Complex I and our understanding of this complex enzyme has slowly been revealed through studies such as electron density mapping [151] and structural characterization at $3.8 \AA$ of Yarrowia lipolytica [152], a cryo-EM map of bovine Complex I [152], a crystal structure of the membrane arm of E. coli at $3.0 \AA[153,154]$, a crystal structure of initially the peripheral arm of $\mathrm{T}$. thermophiles [155] and finally this year the entire crystal structure of Complex I at $3.3 \AA$ resolution [140]. These studies have revealed that the key structures of Complex I is well preserved and therefore forms the basis of our understanding of the structure and function of the human form of the enzyme.

The $\mathrm{L}$ shaped structure of Complex I comprises of the NADH-oxidizing dehydrogenase module ( $\mathrm{N}$ module) which is connected to the $\mathrm{Q}$ module. The $\mathrm{N}$ nodule contains an NADH oxidation site with a FMN molecule, which accepts electrons and feeds them into a chain of Fe-S clusters. The $\mathrm{Q}$ module contains a ubiquinone reduction site and electrons from the $\mathrm{N}$ module are passed to this site [155]. The membrane arm consists of the p-module, which translocates protons and the subunit $\mathrm{NuOH}$ (in E.coli and $\mathrm{Nqo8}$ 
in Thermus spp) which is the interface to the peripheral arm [156]. The two arms of Complex I are functionally and evolutionarily independent apart from the at their interface where quinone binds [157]. Figure 1.5 demonstrates the structure of Complex I. 


\section{Figure 1.5}

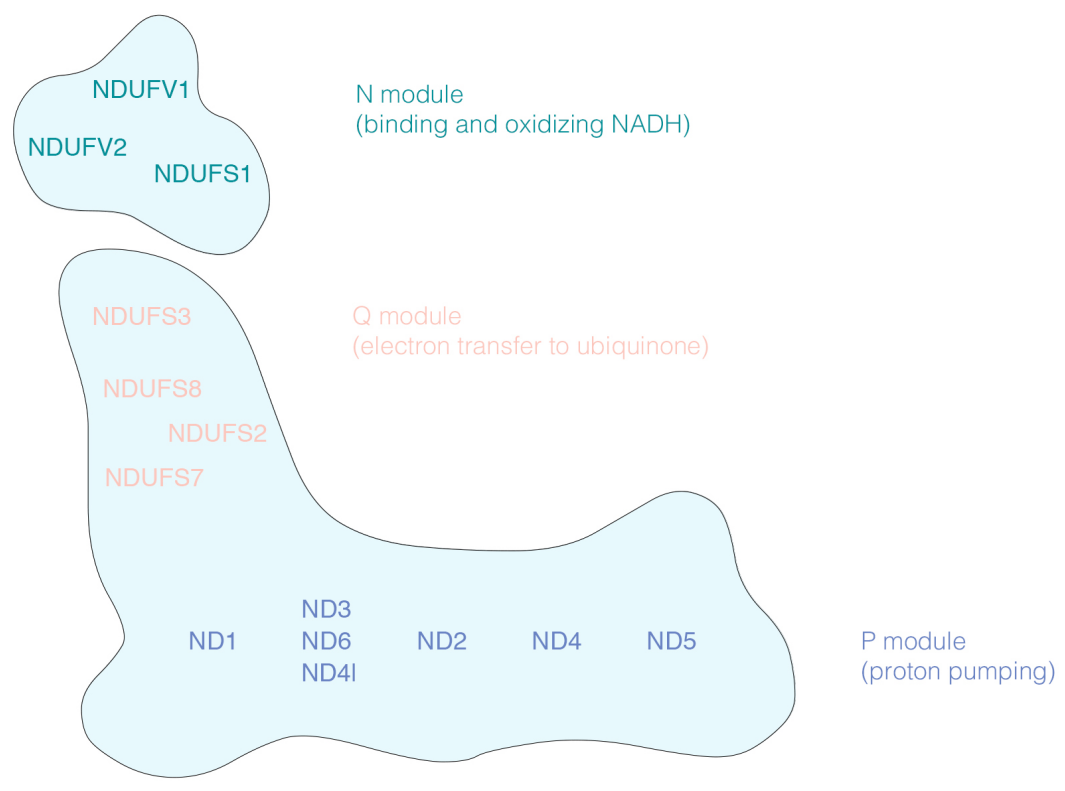

Figure 1.5. Structure of Complex I and the core subunits. Complex I is an L-shaped structure composed of a peripheral arm and a membrane arm. The membrane arm consists of the $\mathrm{P}$ module where the mitochondrial encoded core subunits are situated and the peripheral arm is made up of the $\mathrm{N}$ module and $\mathrm{Q}$ module made up from the nuclear encoded core subunits. Adapted from Mimaki et al (2012): Understanding mitochondrial Complex I assembly in health and disease. Adapted from Mimaki M et al. (2011) Biochimica et Biophysica Acta [158] 
Bacterial Complex I has 14 strictly conserved core subunits (Nqo1-Nqo14), which together are vital in effective Complex I function [140, 159]. The peripheral domain in $T$. thermophilus contains nine core subunits Nqo1-6, Nqo9, frataxin-like Nqo15 and possibly the chaperone Nqo16. The peripheral domain also contains FMN, 8-9 Fe-S clusters and finally quinone with its binding site at the junction with the membrane domain. The membrane arm is composed of seven subunits Nqo7, Nqo8 and Nqo1-14 (Nq012-14 are known as antiporter-like) [139].

Bovine and human Complex I is made of 45 subunits and during the course of evolution 30 additional supernumerary subunits were acquired resulting in a complex with a total molecular weight of $980 \mathrm{kDa}[143,160]$. There are seven mitochondrial encoded subunits ND1-6 and ND4L, all of which are core subunits [149]. There are 38 nuclear encoded subunits, which are transported into the mitochondria of which NDUFV1, NDUFV2, NDUFS1, NDUFS2, NDUFS3, NDUFS7 and NDUFS8 are core subunits. Over time, mammalian Complex I acquired 30 supernumerary subunits, the role of these has yet to be fully elucidated $[147,161]$ but is thought to involve biogenesis and stability of Complex I [147]. . Table 1.4 outlines the core subunits of Complex I in E.coli, $T$ thermophilus and the equivalent human subunits. 
Table 1.4

\begin{tabular}{|c|c|c|c|}
\hline Module & Homo Sapiens & E.coli & T.thermophilus \\
\hline \multicolumn{4}{|c|}{ Peripheral arm } \\
\hline \multirow[t]{3}{*}{ N-module } & NDUFS1 & NuoG & Nqo3 \\
\hline & NDUV1 & NuoF & Nqo1 \\
\hline & NDUFV2 & NuoE & Nqo2 \\
\hline \multirow[t]{4}{*}{ Q-module } & NDUFS2 & NuoD & Nqo4 \\
\hline & NDUFS3 & NuoC & Nqo5 \\
\hline & NDUFS8 & Nuol & Nqo9 \\
\hline & NDUFS7 & NuoB & Nqo6 \\
\hline \multicolumn{4}{|c|}{ Membrane arm } \\
\hline \multirow[t]{7}{*}{ P-module } & ND1 & $\mathrm{NuoH}$ & Nqo8 \\
\hline & ND2 & NuoN & Nqo14 \\
\hline & ND3 & NuoA & $\mathrm{Nqo7}$ \\
\hline & ND4 & NuoM & Nqo13 \\
\hline & ND4L & NuoK & Nqo11 \\
\hline & ND5 & NuoL & Nqo12 \\
\hline & ND6 & NuoJ & Nqo10 \\
\hline
\end{tabular}

Table 1.4 The core subunits of Complex I in different species.

Adapted from Sazanov L et al. (2014) Nat Rev [258] 


\subsubsection{Electron transfer, proton translocation and the link between these processes}

The first step in $T$ thermophilus oxidative phosphorylation involves NADH binding to a pocket in Nqo1 in the peripheral domain of $\mathrm{Cl}$ allowing transfer of hydride ions to FMN followed by the transfer of one electron at a time to the Fe-S clusters and finally two electrons are transferred to the quinone binding site formed between subunits $\mathrm{NqO} 4$, $\mathrm{Nqo6}, \mathrm{Nqo} 7$ and Nqo8 at the interface between the peripheral domain and membrane domain [139]. The antiporter-like subunits in the transmembrane domain allow proton pumping due to the fact that they possess five helices which form a cytoplasmic half and periplasmic half channel [139].

The mechanism that links electron transfer to proton translocation has not been elucidated and several models have been proposed including the direct model where there is direct binding through protein binding sites or the indirect model which proposes conformational changes to the enzyme [162]. The recent study solving the entire structure of Complex I in T thermophilus gave strength to the model that the mechanism most likely involves long-range conformational change. The proposed mechanism is that conformational changes at the interface of the matrix and membrane domains may drive proton translocation by moving the helical structure of the membrane domain into motion. [140, 163].

The actual assembly of Complex I has been subject to numerous studies in many different types of organism but a conclusive model of how this takes place has yet to be established due to the complexity of the components that make up this enzyme and the fact that it is under both mitochondrial and genomic control [158]. A few studies have been conducted to ascertain the function of some of the Complex I subunits in the assembly process [164, 165]. For example, Yadava et al demonstrated in Chinese hamster cell lines the importance of the nuclear subunit NDUFA1 in the Complex I assembly process [165]. Experiments based on cell derived form Complex I deficient patients has revealed that the matrix and membrane arm of the complex are probably present together as intermediates early in the formation of the enzyme but this is in opposition to the model elucidated in $N$. crassa where the two arms are assembled independently [166]. 
Complex I deficiency is the most common of all respiratory chain defects [167]. Studies have revealed that mutations within all of the 14 core Complex I subunits, both mitochondrial and nuclear [168-170], can lead to Complex I deficiency and a heterogeneous group of clinical manifestations related to defects in energy metabolism. The most common mitochondrial disorder is the lethal infantile Leigh syndrome but other clinical presentations include adult-onset neurodegenerative disorders and myopathies [171, 172]. Ugalde et al examined fibroblasts from 15 patients with known mutations within nuclear encoded Complex I genes and demonstrated by isolating mitochondrial particles from these samples and carrying out $\mathrm{BN}$-polyacrylamide gel separation of multisubunit complexes that these patients had decreased levels of intact Complex I and a corresponding decrease in Complex I activity using an in-gel activity assay. Furthermore, there was also a decrease in Complex III levels in patients with mutations in NDUFS2 and NDUFS4 [173]. Several other groups have described deficiencies in Complex I and III simultaneously in patients with specific mutations in Complex I genes [174]. The converse observation of Complex I deficiency in a patient with Complex III mutations has also been demonstrated [175]. Further analysis investigating Complex I and III deficiency have shown that there is likely a physical interaction between these complexes, which could explain why they can affect the stability of one another [174, 176]. The interaction of mitochondrial respiratory chain complexes has been investigated in several species and the concept of an association to form higher supramolecular structures called supercomplexes or respirasomes has been established. Schagger et al carried out experiments in bovine heart mitochondria and established the association of Complex I+III and Complex I+III+IV [177]. Furthermore, similar experiments in human mitochondria revealed supercomplexes of Complex I+III and I+III+IV but only Complex I+III was required for the stability of other respiratory complexes $[178,179]$.

Although the role of the supernumerary complexes is unclear, mutations within these genes with a corresponding decrease in Complex I function have also been identified. Francis et al have demonstrated in a mouse model of Alzheimer's disease that these mice had decreased expression of the supernumerary Complex I subunit NDUFB8 and this was accompanied by a decrease in Complex I and Complex III activity [180]. 


\subsection{Synthetic lethality}

\subsubsection{The concept of synthetic lethality}

A major challenge in cancer therapeutics is to develop specific therapies to target genetically distinct tumour types. The potential of therapy based on targeting the underlying genetic defects driving cancer is that it may cause selective killing of tumour cells while sparing normal cells, resulting in both increased efficacy and reduced toxicity. The identification of synthetic lethal interactions represents an attractive approach for targeting many of these defects. Two genes are said to have a synthetic lethal relationship if a mutation in either gene alone is compatible with viability but a mutation in both results in cell death [181]. The concept of synthetic lethality has been used in the clinical setting with PARP inhibitors in the treatment of BRCA deficient tumours. PARP1 is an important member of the BER pathway but PARP1-/- mice have been found to be viable $[182,183]$. In the absence of PARP1 the increased formation of SSBs and collapsed replication forks has been shown to trigger the HR pathway to maintain genetic stability. Tumours deficient in the tumour suppressor genes BRCA1 and BRCA2 which are key members of the HR pathway are highly sensitive to PARP inhibition [184]. The mechanism behind this synthetic lethal relationship has been established to be due to the fact that BRCA deficient tumours are unable to carry out DNA damage repair through the HR pathway when treated with PARP inhibitors leading to unrepaired collapsed replication forks and cell death [185]. In an analogous fashion to using inhibitors of the DNA repair protein, PARP, in the treatment of patients with germline mutations in the tumour suppressor genes BRCA1 and BRCA2, targeting genes that are synthetically lethal with MMR deficiency could result in selective killing of MMR deficient cells and therefore could be therapeutically beneficial.

\subsubsection{Synthetic lethality and the MMR pathway}

Our group is interested in identifying synthetic lethal targets with MMR deficient cancers and have shown that silencing of the mitochondrial DNA polymerase POLG (involved in the repair of 8-oxoG lesions in the mitochondria) is synthetically lethal with MLH1 deficiency [79]. This synthetic lethal interaction was associated with an increase in oxidative DNA lesions (8-OxoG) in the mtDNA only. Strikingly, MSH2 deficient cells were not sensitive to POLG silencing, but showed selectivity with depletion of the 
nuclear polymerase POLB, which was associated with an increase in nuclear 8-oxoG (Figure 1.3) [186]. The authors also established that there was a decrease in mtDNA integrity in the MLH1-deficient cells compared to the proficient cell lines. Furthermore, by fractionating cellular protein into mitochondrial and nuclear components, MLH1 was found to be expressed in both the mitochondria and nucleus but MSH2 was only expressed in the nucleus [79]. Other mitochondrial proteins not involved in DNA repair, namely the kinases CKMT2, PCK2, and PINK1 (Figure 1.3) were also found to be synthetically lethal with deficiencies in a range of MMR genes (MLH1, MSH2 and MSH6) due to an accumulation of both nuclear and mitochondrial oxidative DNA damage [187]. Using a similar approach, our group screened a compound library and established that MSH2-deficient cell lines in vitro and in vivo are selectively lethal to treatment with the oxidative damage-inducing agent, methotrexate [188]. These findings have been taken into the clinic as a Phase II clinical trial (ClinicalTrials.gov Identifier: NCT00952016) in the metastatic colorectal cancer population, at the Royal Marsden Hospital, London.

\subsubsection{Synthetic lethality and cancer cell metabolism}

The concept of using synthetic lethal approaches to target cancer cell metabolism is gaining an increasing amount of interest. Several metabolic enzymes with somatic mutations have been identified in cancer cells leading to research identifying targets to achieve selective cell death in these cells. The tumour suppressor fumarate hydratase has been found to be synthetically lethal with several genes in the heme biosynthesis pathway [189] as well as with adenylate cyclase [190]. Argininosuccinate synthase 1 (ASS1) is the rate-limiting enzyme for arginine biosynthesis and is known to be deficient in a range of tumour types including approximately $50 \%$ of malignant pleural mesotheliomas (MPM) and malignant melanomas [191]. Synthetic lethal targeting of ASS1-deficient tumours with the arginine-depleting agent ADI-PEG20 has been found to be a useful therapeutic strategy in vivo and has been tested with promising results in metastatic melanoma [192] as well as in an ongoing study in MPM (ClinicalTrial.gov Identifier: NCT01279967) [193]. More recently Locke et al investigated resistance mechanisms in ASS1-deficient tumours and generated an MPM cell line resistant to ADI-PEG. The investigators identified that this cell line and serum from patient samples with ASS1-deficient MPM displayed a synthetic lethal relationship with polyamine 
metabolism (decreased levels of acetylated polyamine metabolites and increased expression of polyamine biosynthetic enzymes) which could be exploited in ADI-PEG20 resistance [194]. The AMPK agonist Metformin has been found to have a synthetic lethal relationship with glucose starvation in vitro highlighting the potential for exploiting this metabolic synthetic relationship [195]. The drug Dichloroacetate has been found to selectively kill cancer cells in solid tumours through enhancing tumour hypoxia by increasing pyruvate consumption in the mitochondria and total oxygen consumption [196]. Interfering with tumour bioenergetics by using a combined drug approach of treating cancer cells in a mouse xenograft model with metformin and the glycolysis inhibitor 2-deoxy-D-glucose (2DG) which both interfere with energy pathways causes selective cell death in these cells [197]. High throughput siRNA [190, 198], drug and genetic screens [199] have identified a range of potential metabolic targets in cancer cells including the metabolic enzymes isocitrate dehydrogenase 1 [200], fumarate hydratase and succinate dehydrogenase [189, 201]. 


\section{Figure 1.3}

A

Synthetic lethal targing of MSH2

MSH2 proficient

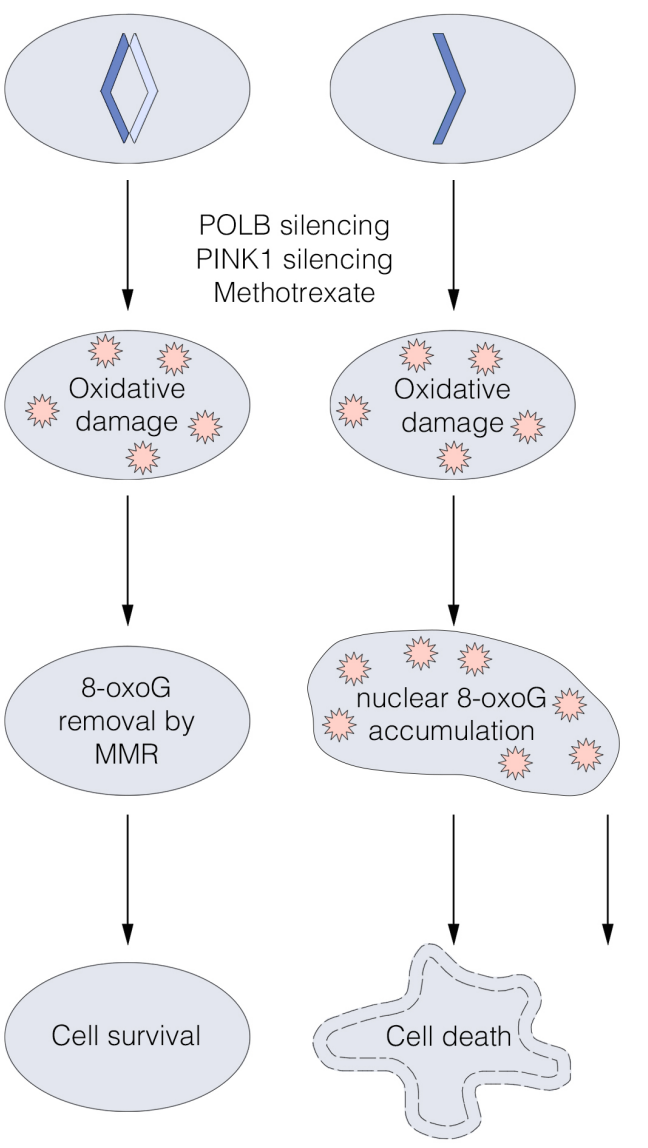

$\mathrm{MSH} 2-\langle-\mathrm{MSH} 6$

MutSa
MSH2 deficient

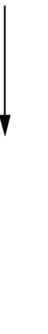

B

Synthetic lethal targing of MLH1

MLH1 proficient

MLH1 deficient
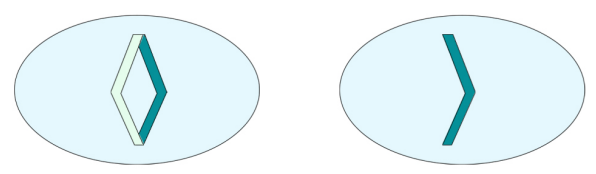

POLG silencing PINK1 silencing

$\checkmark$
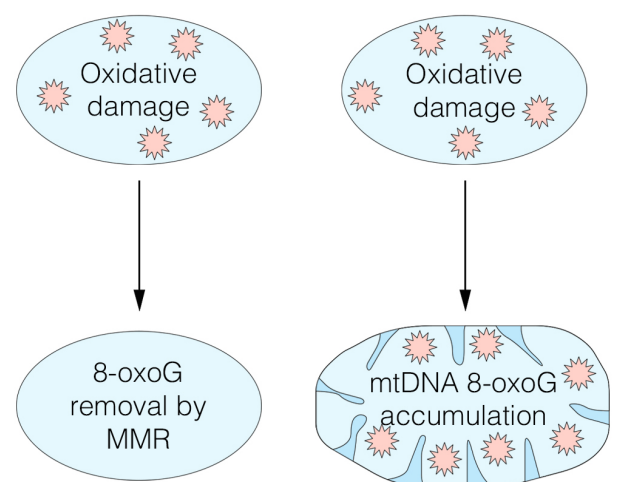

8-OxoG

MMR
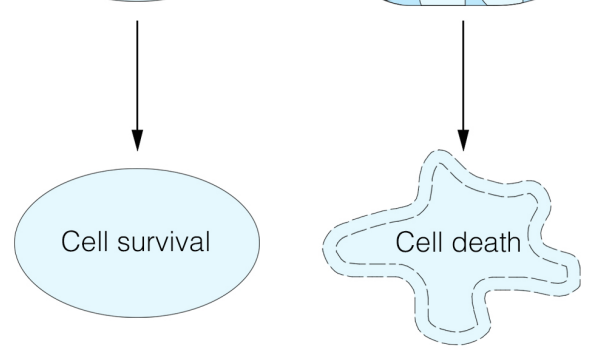

$\mathrm{MLH} 1-\bigcup_{\text {MutLa }}^{\text {PMSH3 }}$ 
Figure 1.3: Accumulation of oxidative DNA damage causes synthetic lethality in MSH2 and MLH1-deficient cells.

(A) Silencing of the genes PINK1, POLB or treatment of cells with the chemotherapeutic drug, methotrexate leads to selective cell death in $\mathrm{MSH} 2$ deficient cell lines through increased oxidative DNA damage (8-oxoG lesions in DNA) which can be successfully repaired in MMR proficient cells. (B) Silencing of PINK1 or the mitochondrial gene POLG causes selective cell death in only the MLH1-deficient cell lines and not the MSH2 deficient cells. More specifically, in $\mathrm{MSH} 2$ deficient cell lines $(\mathrm{A})$ there is an accumulation of nuclear oxidative DNA lesions and in (B) MLH1-deficient cells 8-oxoG lesions accumulate in the mitochondrial DNA leading to cell death. Adapted from Bridge, G et al. (2014) Cancers [202] . 


\subsection{Oxidative DNA damage}

\subsubsection{Reactive oxygen species (ROS)}

ROS is the term used for a group of molecules containing oxygen and are generally known to have reactive properties [203]. Cellular components, such as lipids, proteins and nucleic acids, are continuously exposed to endogenous and exogenous ROS, which potentially pose a threat to the stability of the genome. ROS are divided into two main groups: radicals and non-radicals. The radicals include the most reactive species, which is the oxygen-derived hydroxyl radical $\left(\mathrm{HO}^{\circ}\right)$ as well as the intermediate or weakly reactive species, the superoxide anions $\left(\mathrm{O}_{2}{ }^{\circ}\right)$, hydroperoxyl radicals $\left(\mathrm{HO}_{2}{ }^{\circ}\right)$, peroxyl radicals $\left(\mathrm{RO}_{2}{ }^{\circ}\right)$, alkoxyl radicals $\left(\mathrm{RO}^{\circ}\right)$, nitric oxide $\left(\mathrm{NO}^{\circ}\right)$ and singlet oxygen $\left({ }^{1} \Delta_{\mathrm{g}} \mathrm{O}_{2}\right)$ Nitric oxide is itself not reactive but can react with $\mathrm{O}_{2}{ }^{--}$to form peroxynitrite (ONOO-) and eventually its protonated form peroxynitrous acid $(\mathrm{ONOOH})$, which can in turn yield the highly reactive $\mathrm{HO} \cdot$ or $\mathrm{NO}_{2}{ }^{\cdot}$ [204]. The main non radical is hydrogen peroxide $\left(\mathrm{H}_{2} \mathrm{O}_{2}\right)$ which is generally weakly reactive but can react with transition metal ions to form the more reactive $\mathrm{HO}^{\circ}[205]$.

The majority of ROS found in aerobic cells are generated during normal cellular metabolism in processes, which require oxygen, mainly in the mitochondria, peroxisomes and endoplasmic reticulum [203]. The mitochondria has been established to be the main site of ROS production since $2 \%$ of the oxygen used by the mitochondria becomes reduced to generate superoxides [203]. The tricarboxylic acid cycle (TCA) is composed of several enzymes in the mitochondrial matrix, which pass electrons from intermediary metabolites to the electron transport chain (ETC). The ETC has the potential to form ROS at several points in the pathway as electrons are passed through the series of enzymes using flavin adenine dinucleotide (FAD) or flavin mononucleotide (FMN) as carriers [206]. ROS is generated when electrons become 'stuck' on the flavin group. There are many mitochondrial proteins aside from those of the TCA cycle which use flavin containing groups and there is potential for ROS formation at all these sites [207]. Some enzymes also use iron-sulphur clusters as electron carriers and ROS can also be formed here in a similar manner to that described above with flavin containing groups [208]. The energy source for all cells, ATP, is generated in the mitochondria through oxidative phosphorylation. This involves electrons generated from the TCA 
cycle and glycolysis being passed from $\mathrm{NADH}$ and $\mathrm{FADH}_{2}$ along a series of five protein complexes in the ETC [135]. The ETC is inherently leaky and electrons can escape and form $\mathrm{O}_{2}$ when they become trapped by $\mathrm{O}_{2}$. It is thought that the main sites of ROS generation are at Complex I, II and III since these are proton pumps [207]. Furthermore, Ubiquinone (coenzyme $Q$ ) is also a site for ROS generation since this inner membrane quinone transports electrons to Complex III from various sources including Complex I and II [207]. Certain enzymes can generate ROS as a result of the reactions they catalyze, these include NADPH oxidase, arachidonic acid and several metabolic enzymes including cytochrome p450 enzymes [203]. ROS can also be produced as part of the inflammatory response by neutrophils, eosinophils and macrophages [209]. In addition, ROS may also be generated by exogenous agents such as UV exposure, ionising radiation, carcinogenic compounds and redox-cycling drugs [210].

Maintaining optimum ROS levels is essential for cellular homeostasis since there is a fine balance between the levels of ROS required for cell signaling, proliferation, differentiation, activation of the inflammatory response and the excess ROS levels that are deleterious for DNA, protein and lipids [203].

ROS species cause oxidative DNA damage which is a term used to describe the resulting damage when ROS alter DNA bases and sugar backbones resulting in DNA modifications [205]. The antioxidant defense system, which is the first line of defense protecting cellular components from ROS, is described in detail later in this introduction chapter. The majority of superoxide anions are converted into the less reactive $\mathrm{H}_{2} \mathrm{O}_{2}$ by superoxide dismutases (SOD) but a proportion may react with nitric oxide to form ONOO-, a very strong oxidant [211]. Superoxides which have managed to evade dismutation can form hydroxyl radicals through the Harber-Weiss reaction and $\mathrm{H}_{2} \mathrm{O}_{2}$ can also form the highly reactive hydroxyl species by reacting with $\mathrm{Fe}^{2+}$ [211]. Hydrogen peroxide is highly diffusible through different cellular compartments but possesses low chemical reactivity and therefore is only directly responsible for modifying proteins via thiol groups [209]. The $\mathrm{HO}^{\circ}$ is the oxidant species that poses the biggest threat to DNA, given that it reacts with both purine and pyrimidine bases and the sugar moiety of the DNA backbone. Furthermore there are to date no known enzymes or other neutralizing molecules that can combat $\mathrm{HO}^{\circ}$. [205]. 


\subsubsection{ROS and oxidative DNA damage}

The hydroxyl radical causes DNA damage by its ability to add to the double bonds of DNA bases as well as remove hydrogen from thymine and the $\mathrm{C}-\mathrm{H}$ bonds of 2'deoxyribose [212]. Additional reactions with purines generate $\mathrm{C} 4-\mathrm{OH}-, \mathrm{C} 5-\mathrm{OH}-$ and $\mathrm{C} 8$ $\mathrm{OH}$-adduct radicals of guanine and adenine [213] and reactions with the pyrimidines form $\mathrm{C} 5-\mathrm{OH}$ - and $\mathrm{C} 6-\mathrm{OH}$-adduct radicals of thymidine and cytosine [214]. Thymidine can also form an allyl radical when a hydride anion $\left(\mathrm{H}^{\circ}\right)$ is removed from its methyroup [215]. Downstream oxidation and reduction reactions of these radical intermediates result in a plethora of DNA lesions, including the most studied of oxidative lesions, the purine derivatives 8-oxoguanine (8-oxoG), 8-OH-Ade , 2,6-diamino-4-hydroxy-5formamido-pyrimidine (FapyGua), and the oxidised pyrimidines cytosine glycol and thymine glycol (Figure 1.4) [205]. The intermediary lesions resulting from oxidative DNA damage repair such as apurinic and apyrimidinic (AP) sites also pose a threat to the cell [216]. The hydroxyl radical can also attack all five carbons of 2'-deoxyribose and further processing of these lesions yields products incorporated within DNA, released from DNA or broken DNA strands bound to DNA. These lesions then have the potential to form DNA strand breaks [205, 209]. 8-oxoG is the most stable DNA lesion and can mispair with adenine $[124,125]$ and less frequently it can pair with the original cytosine [217] during DNA replication. Increased 8-oxoG levels have been reported in numerous in vitro and in vivo experiments when cultured cells and animals are exposed to oxidative stress [216]. The pairing of 8-oxoG and FapyGua with adenine has been shown to be pre-mutagenic and result in the mutagenic G:T transversions both in vivo $[218,219]$ and in vitro [220]. On the other hand, the less frequent event of 8-oxoG pairing with cytosine has not been shown to result in mutations [221]. Kalaman et al have shown that in Simian kidney cells FapyGua result in G:T transversions more frequently than 8-oxoG [217]. The other purine derived molecule 8-OH-Ade can pair with thymine as well as mispair with guanine and adenine [222] but the resulting mutations have been shown to be weakly mutagenic in simian kidney cells [217] and have been shown to cause $A: G$ and $A: C$ transversions mutations at a rate of approximately $1 \%$ [223]. Deamination and dehydration of the pyrimidine cytosine glycol to form 5-OH-Cyt and uracil glycol to form 5-OH-URa which can then mispair with adenine, have been found to result in C:T transitions [205]. Thymine glycol is much less mutagenic since it generally pairs with cognate adenine [224] but can much less 
frequently pair with guanine to result in $\mathrm{T}: \mathrm{C}$ transitions [225]. Feig et al investigated the lesions that occur as a result of oxidative DNA damage by developing a ROS generating system and then inserting the formed products into DNA containing a target gene for scoring of mutations [226]. They found that the most frequently occurring mutagenic species as a result of oxidative damage was 5-hydroxy-2'-deoxycytidine and this caused C:T transversions in E. Coli at a rate of $2.5 \%$ [226]. It is clear that oxidativeinduced DNA lesions are mutagenic and therefore the antioxidant defense system, as well as DNA repair mechanisms has evolved to try and minimize the damage that ROS and the resulting DNA lesions can cause. 


\section{Figure 1.4}

\begin{tabular}{|c|c|c|c|}
\hline Original DNA base & Oxidatively Induced Product & Repaired By & Proteins Implicated \\
\hline \multirow[t]{7}{*}{ Guanine } & \multirow[t]{4}{*}{ 8-oxoguanine } & BER & OGG1; MUTYH \\
\hline & & MMR & MSH2; MSH6; MLH1 \\
\hline & & NER & Multiple members \\
\hline & & dNTP pool sanitation & MTH1 \\
\hline & 2, 6-dilamino-4hydroxy-5-formamido-pyrimidine & BER & $\begin{array}{l}\text { OGG1; NEIL 1; NEIL 3; } \\
\text { MUTYH? }\end{array}$ \\
\hline & & BER & NEIL 1; NEIL 3 \\
\hline & guanidinohydantoin & BER & NEIL 1 ; NEIL 3 \\
\hline \multirow{4}{*}{ Cytosine } & 5-hydroxycytosine & BER & NEIL 1 ; NEIL 3; NTH1 \\
\hline & 5,6-dihydroxycytosine & BER & NTH1 \\
\hline & & BER & NEIL2; NEIL3; NTH1 \\
\hline & & BER & NEIL 2 \\
\hline \multirow[t]{4}{*}{ Adenine } & 8-hydroxyadenine & $\begin{array}{l}\text { dNTP pool } \\
\text { sanitisation }\end{array}$ & MTH1 \\
\hline & \multirow[t]{3}{*}{ 4, 6-diamino-5-formamidopyrimidine } & BER & NEIL 1 ; NEIL 3 \\
\hline & & dNTP pool sanitisation & MUTYH \\
\hline & & dNTP pool sanitisation & MTH1 \\
\hline \multirow[t]{5}{*}{ Thymine } & 5-hydroxy-6-hydrothymine & BER & NTH1 \\
\hline & \multirow{3}{*}{ thymine glycol } & BER & NEIL1; NEIL3; NTH1 \\
\hline & & MMR & MSH2 \\
\hline & & NER & Multiple proteins \\
\hline & 5-hydroxy-5-methyl-hydantoin & BER & NEIL1; NEIL3 \\
\hline
\end{tabular}

Figure 1.4. Outline of oxidative DNA lesions formed when ROS damage DNA bases and the DNA repair pathways responsible for repairing the resulting DNA damage.

Adapted from Bridge, G et al. (2014) Cancers [202] . 


\subsubsection{Oxidative DNA damage, mutagenesis and cancer}

Oxidative DNA damage is a very common event and therefore it is not surprising that this form of DNA alteration has been studied in relation to the aetiology and progression of cancer. Sequencing of a large range of cancer genomes over several tumour types showed that $\mathrm{C}: \mathrm{T}$ transitions are the most commonly occurring mutation found in human tumours and in the tumour suppressor gene TP53 and a proportion of these mutations can be attributed to oxidative DNA damage [227, 228]. Chronic oxidative stress, especially in the context of chronic inflammation, has been linked to carcinogenesis. One of the most well-described causal relationships with cancer and oxidative stress is that between ulcerative colitis and colorectal cancer. The evidence suggests that cancer-causing genes are altered in this disease due to the production of ROS by inflammatory mediators $[229,230]$. To date many studies examining oxidative DNA lesions in malignant cells or tissues from a wide variety of tumours compared to matched normal samples have found higher levels of 8-oxoG and other markers of oxidative stress in the cancerous samples [231-234]. More specifically, Gackowski et al illustrated that the levels of 8-oxoG by high performance liquid chromatography were significantly higher in lymphocytes from blood samples taken from 43 patients with colorectal cancer 55 control subjects [235]. Furthermore, the levels of (antioxidant vitamins (vitamin E (a-tocopherol), vitamin A (retinol) and vitamin C) was lower in the colorectal cancer samples compared to controls [235]. Olinski et al have shown that the size of benign uterine myomas was positively correlated with levels of 8-oxoG lesions and it is well known that the larger the myoma the greater the risk of malignant transformation [231, 236]. Malins et al have also shown a positive correlation between metastatic potential and levels of oxidative DNA lesions in breast cancer [237].

Nowsheen et al examined the levels of oxidatively induced clustered lesions (OCDLs) across several tumour types compared to match surrounding tissue [238]. In most cases there was an increase in OCDLs in the tumour tissues although the authors did remark on the fact that this was not always in a uniform manner across all samples from the same tumour type. The authors of this study discussed some limitations, which are key to the controversy surrounding the measurement of oxidative DNA lesions in cancer tissue and establishing a conclusive link between these lesions. The authors of this study and many other investigators within this field of research have commented on the 
fact that tumour tissues may have endogenously high background levels of OCDLs due to factors such as impaired DNA repair, attenuated antioxidant capacity, increased cellular metabolism and higher ROS levels due to a chronic inflammatory microenvironment [238, 239]. Alterations within established tumours has been shown to induce an inflammatory environment and in some tumours this inflammatory environment is present before the malignant transformation occurs [240] leading to an accumulation of inflammatory cells and increases in ROS production and oxidative stress [241]. In breast cancer ROS levels have been found to be elevated because of exposure to oestrogen [242]. All the factors mentioned above mean that establishing a direct relationship between oxidative DNA damage and cancer in general is difficult. Investigators within the field of oxidative DNA damage have concluded that at the very least malignancy and oxidative DNA lesions exist in parallel and furthermore most evidence does point to a relationship between the two, but it seems to be context dependent rather than related to cancer as a whole [231].

Furthermore, it is important to note that most investigators are cautious about potential inaccuracies in studies measuring levels of oxidative DNA damage due to the problems with measuring oxidative DNA lesions accurately. It has been estimated by various experimental studies that the background level of oxidative DNA lesions in normal human or animal cells and tissues is anywhere between 100-10,000 lesions/Gbp [241, 243, 244] but controversy still exists about this topic [245]. The most obvious problem with measuring oxidative DNA damage lesions is that DNA extraction inevitably leads to some amount of unavoidable oxidation, which can result in unreliable results [231]. The European Standards Committee on Oxidative DNA Damage (ESCODD) examined the testing of 8-oxoG in various different types of samples in different laboratories using a variety of methods [245]. The main methods available for testing are chromatographic methods (GC-MS, HPLC with electrochemical detection (ECD) or HPLC-MS/MS) and immunoassays. They established that each method had its own weaknesses and strengths but some of the chromatographic methods displayed artefactual oxidation whilst the immunoassays lacked specificity. The study group concluded that there is still need for an alternative validated method to be developed to measure these oxidative lesions [245]. 


\subsection{DNA Repair of Oxidative DNA damage}

BER is largely responsible for the repair of oxidative DNA damage and to a lesser extent NER [209]. Both these pathways differ in the size of lesions that they repair and function by excising DNA lesions before instigating polymerase and ligase activity to fill in the resultant gap [246, 247].

\subsubsection{Base excision repair (BER) repair of nuclear oxidative DNA damage}

Base excision repair is a highly conserved pathway that has evolved to repair non-bulky small nucleotide lesions such as bases that have been incorporated into DNA erroneously or damaged bases such as 8-oxoG [246]. The main steps involved in BER are: removal of the damaged base and creation of an abasic site as an intermediary molecule, end-processing of the DNA, filling the resulting gap with a polymerase and sealing the nick with a DNA ligase [248]. The repair of damaged DNA in BER is divided into short patch (SP) and long patch (LP) repair. The former is involved in the repair process if the removal of the damaged base results in a free 3'hydroxyl on one side which can be filled by a DNA polymerase and a 5' phosphate on the other side to ligate to the new base [249]. Long patch repair is used in circumstances where a 5' end is created, which cannot be ligated to the newly created base [249]. The first step in BER repair is the recognition of the damaged base by DNA glycosylases. The glycosylase will depend on the base to be excised. These include uracil-DNA glycosylase (UNG), 8oxoguanine DNA glycosylase (OGG1), MutY homolog (MUTYH), endonuclease three homolog 1 (NTHI) and NEI endonuclease VIII-like 1, 2 or 3 (NEIL1/NEIL2/NEIL3). These enzymes recognise different single base lesions (Figure 1.4). DNA glycosylases need to recognise damaged bases from undamaged bases to carry out their function [250]. Several mechanisms describing how glycosylases recognise DNA damage have been proposed including the fact that they can easily recognise damaged DNA double helix structures by moving along DNA slowly using a Phe residue as a wedge to look for damaged DNA [251]. However, it is still unclear how these enzymes recognise lesions such as guanine oxidation, which do not change DNA. Ultimately to actually reach the damaged base the glycosylases scan along and gently pinch the DNA causing it to bend at the site of a damaged base, which flips out of the double helix and enters the binding site of the enzyme. Subsequently the $\mathrm{N}$-glycosidic bond between the damaged 
base and the 2'-deoxyribose is cleaved, generating an apurinic- or apyrimidinic- (AP) site [252]. For SP-BER the AP-sites must be further processed by an endonuclease, most commonly APE1, to create the correct 3' and 5' ends that allow DNA polymerase beta $(P o \mid \beta)$ in the nucleus to remove the remaining sugar backbone of the damaged DNA base using its dRP lyase activity [246]. After this step de novo DNA synthesis can take place from the 3' hydroxyl group and finally DNA ligase I (LIG1) can ligate the nascent base. SP-BER only allows the incorporation of a single nucleotide. The LPBER allows the incorporation of multiple bases [249] and nucleotide synthesis occurs by Pol $\beta$, Pol $\delta$ and Pole in association with PCNA [253]. A substrate which can be ligated is created by these polymerases forming a 'flap intermediate' at the 5' end of the DNA which is then removed by the nuclease flap endonuclease 1 (FEN1) resulting in a ligatable substrate [254]. There are numerous factors determining whether SP-BER or LP-BER takes place and these include the ATP concentration available after POLB has exerted its lyase activity since high concentrations usually lead straight to ligation but low concentrations of ATP are less likely to favour ligation Other factors include the initial type of lesion, stage of the cell cycle and certain lesions are resistant to Pol $\beta$ activity [249]. The nick in the DNA is sealed by LIGI in the case of LP-BER and LIGIIIa/XRCC1 in SP-BER [205, 248, 255].

\subsubsection{Nucleotide excision repair (NER) and nuclear oxidative DNA damage}

Whilst BER acts to repair individual nucleotides, the NER system generally removes large DNA-distorting lesions such as intra-strand crosslinks, tandem lesions and bulky adducts [205]. NER has been described to repair certain single nucleotide oxidativelyinduced lesions such as thymine glycol [256], 8-oxoG [257] and 8,5'-cyclopurine-2'deoxynucleosides [258, 259], the latter of which cannot be repaired by BER due to the 8,5'-covalent bond. Nucleotide excision repair has two main mechanisms by which it carries out repair: global genome repair (GGR-NER) which is associated with repair of the entire genome and transcription coupled repair (TCR-NER) for the repair dedicated to transcribing repair strands. This occurs either during normal surveillance of the genome or specifically on transcribing DNA strands [260]. The primary step in NER is the recognition of the site of damage and this is carried out in the GGR-NER pathway by the protein complex composed of XPC-hHR23B together with XPE. In TCR-NER the damage recognition signal occurs when RNA polymerase II (RNAPII) is stalled by a 
DNA lesion and when the Cockayne syndrome proteins (CSA and CSB) are present [261]. The subsequent steps in both the GGR-NER and TCR-NER pathways are similar. Once the section of damaged DNA has been identified the XPC-hHR23B complex brings TFIIH to the site of DNA damage. Also, for both pathways a protein complex of XPA-RPA is formed and is necessary for recruiting the downstream protein necessary for NER repair. XPA-RPA in association with TFIIH and its two helicase subunits XPB and XPD enable the unwinding of the DNA helix around the damaged lesion[262]. Two incisions either side of the lesion are made by the endonuclease activity of XPG cutting 3 ' to the lesion and a complex composed of XPF-ERCC1, XFG and XPA-RPA cutting 5 ' to the lesion [263]. In a similar fashion to BER, the final steps of excision and DNA synthesis to fill the single strand gap is carried out by polymerases ( $\delta$ and /or $\varepsilon$ ) and the resulting gap at the $3^{\prime}$ is filled by DNA ligase I [205, 264]. The single base lesions repaired by BER and NER are highlighted in Figure 1.4

The DNA glycosylases involved in oxidative DNA damage repair have been shown to target specific oxidative DNA lesions [248]. Various in vitro and in vivo studies have shown that the nuclear human OGG1 and the mitochondrial targeted $\beta$-hOgg1 have a major role in the recognition of 8-oxoG. Although studies in E.Coli demonstrated that OGG1 also recognises FapyGua lesions, this was not found to be the case in eukaryotes [265]. Mambo et al investigated 8-oxoG levels in 18 lung cancer and three control cell lines and found overexpression of this hOGG1 in most cases but two of the eighteen tumour samples had decreased protein and mRNA expression of OGG1 compared to the normal samples [266]. Furthermore the two samples with low levels of OGG1 displayed a significant attenuation of 8-oxoG removal in both nuclear and mitochondrial extracts. Despite these findings, Ogg $1^{-/}$mice did not exhibit tumour formation even though the levels of 8-OxoG and G:T transversions were increased [266]. The DNA glycosylases NEIL1 and NTH1 were investigated by developing Neil1/-, $N$ th $1^{-/}$and double mutant mice. During the second year of the experiment up to $16 \%$ of the Neil1 $1^{-/}$and $N$ th $1^{-/}$mice developed tumours and in the double mutant, mice $75 \%$ developed tumours [266]. Furthermore, when oxidatively induced lesions were measured in these animals, the levels of FapyAde (in the liver, kidney and brain) and FapyGUA (liver and kidney) but not 8-oxoG was raised in the the Neil/1/ and double mutant animals. The $\mathrm{Nth} 1^{-/}$mice only displayed an accumulation of FapyAde in the kidneys [267]. To date, the role of the other NEIL proteins in oxidative DNA damage and 
cancer has not been studied in detail [205]. Mice that lack the DNA glycosylase Ape1 are characterised by early embryonic lethality and the Ape1 heterozygotes exhibit increased oxidative stress, carcinogenesis and decreased survival pups [268-270]. Several studies have demonstrated the overexpression of APE1 in tumour tissue and furthermore its association with treatment resistance [271-273]. Many variants of APE1 exist and there are some studies suggesting that certain variants are associated with cancer $[274,275]$ but to date there is no conclusive evidence linking carcinogenesis to a particular variant $[276,277]$. Mutations in the nuclear DNA polymerase $P o / \beta$ have been shown to present in a range of human tumours [278-280]. Structural studies of Pol $\beta$ have revealed that it binds to 8-oxoG at its active site. It has been found to insert both cytosine and adenine depending on the sequence context [281]. There is a paucity of studies looking at the role of Pol $\beta$ with other oxidative DNA lesions. Several polymorphisms of Pol $\beta$ have been linked to certain types of cancer [280, 282] and to OS in pancreatic adenocarcinoma. Li et al demonstrated an improved survival (35.7 months versus 14.8 months) for patients carrying at least one of the two homozygous variant Po/ $\beta$ GG or CC genotypes compared with those carrying the AA/AG or TT/TC genotypes in 378 human pancreatic adenocarcinomas[283].

\subsection{MMR and oxidative DNA damage}

It is widely accepted that BER and NER are the primary DNA repair pathways responsible for the repair of oxidative DNA damage [205]. There is however a growing body of evidence implicating the MMR system in the repair of oxidative DNA damage and this is possibly linked to carcinogenesis due to an accumulation of oxidative DNA damage in the context of MMR deficiency [284, 285].

An early study in 1998 in mice demonstrated that mouse embryonic stem (ES) cells deficient in $\mathrm{MSH} 2$ (Msh2+/- and Msh2 -/-) had much higher levels of genomic 8-oxoG both at baseline and in response to low-levels of ionizing radiation, compared to wildtype controls [286]. A subsequent study by Colussi et al showed that baseline and $\mathrm{H}_{2} \mathrm{O}_{2}$ induced 8-oxoG levels were much higher in extracted DNA from MSH2 deficient mouse embryo fibroblasts (MEFs), MSH2 deficient cells lines and MLH1-deficient cell lines [287]. The authors concluded that this observation was due to impaired removal of 8- 
oxoG from the oxidised dNTP pool incorporated into DNA. Furthermore, overexpressing the hydrolase MTH1 that normally sanitizes the dNTP pool of 8-oxoGTP, reduced the levels of oxidative DNA lesions in the MMR-deficient mouse cells, in comparison to the proficient cells. MTH1 over expression also resulted in a decrease in the mutator phenotype in MSH2 deficient MEFs [287].

Another study that adds to the current evidence implicating the MMR pathway in the repair of oxidative DNA damage was carried out in Helicobacter pylori (H. pylori) since the inflammatory stress response that occurs after infection by this organism in the gastric mucosa results in an increase in ROS [205]. The investigators showed that mutS mutant variant $H$.pylori were more sensitive to ROS inducing drugs including $\mathrm{H}_{2} \mathrm{O}_{2}$ and had increased 8-oxoG accumulation when exposed to oxidative stress, in comparison to wild type mutS [288]. Mazurek et al. further elucidated the distinct roles of the MMR proteins in oxidative repair by showing that MutSa, but not MutS $\beta$ is activated by the presence of a mismatched 8-oxoG lesion [289]. Furthermore, MutSa was able to bind different mismatches with differing affinities: 8-0xoG/T>8-0xoG/G>8-0xoG/A>8$\mathrm{OxOG} / \mathrm{C} \approx \mathrm{G} / \mathrm{C}$. This finding illustrated that in the context of oxidative DNA damage, MutSa is only activated by mismatched DNA. They also demonstrated that this activation occurred because binding of the mismatched DNA caused ATP-ADP exchange due to ATPase activity of MutSa. Given the observed variations in the ability of MutSa to recognize different mismatch lesions, it is thought that the MMR system will continually excise an 8-oxo-G lesion and re-synthesise new DNA until a $C$ is inserted opposite the lesion because MMR is not activated by an 8-oxo-G/C pair [289].

As mentioned above, we have observed a synthetic lethal interaction upon increased oxidative DNA damage in MMR deficient cells, further suggesting a role for the MMR proteins in the repair of oxidative damage $[79,187]$

\subsubsection{BER and MMR}

Studies in yeast and in vivo work have shown that both the BER and the MMR pathway contribute to the repair of oxidative DNA damage. The experiments in S. cerevisiae by $\mathrm{Ni}$ et al identified increased mutation rates due to G:C-to-T:A transversions in combined MMR and BER mutants (msh2/msh6+ogg1) compared to the single ogg1 mutant [290]. 
Russo et al found increased 8-oxoG lesions in the spleen, liver, heart, lungs, kidneys and small intestine of $M s h 2^{-/-}$mice compared to their wild-type controls [285]. A more recent study conducted by the same group aimed to investigate whether MUTYH (a BER protein) and $\mathrm{MSH} 2$ have overlapping or independent roles found a disparity in in vitro compared to in in vivo models. There was no difference in the levels of 8-oxoG lesions in double mutant MEFs in vitro but there was a synergistic increase in 8-oxoG in some of the organs of double mutant animals compared to single mutants. The authors concluded that in vivo, MSH2 and MUTYH act independently and both contribute significantly to the repair of oxidised lesions. They proposed that the disparity seen in vivo compared to in vitro is due to the higher percentage of proliferating cells in vitro. Gu et al conducted a study to allow a more in-depth understanding of how the MMR system and BER may be working together which concluded that MUTYH physically associates with MutSa via MSH6. In addition, they demonstrated that the role of MutSa is to enhance the BER mediated excision of 8-oxoG/A mismatched lesions. The authors speculate that protein-protein interactions may be how the BER and MMR make contact to reduce replicative errors caused by oxidative damage [291].

\subsubsection{MMR and oxidative damage: relevance to carcinogenesis}

Given the dual function of MMR in removing both incorrectly placed bases and oxidatively damaged nucleotides, it is inherently difficult to dissect a separate impact of these two roles upon cancer development. The mutator phenotype is clearly the driving force behind carcinogenesis in many MMR deficient tumours but does reduced 8-oxoG removal also contribute, either via increased MSI or independently?

Few studies have examined the specific role of oxidative damage repair by the MMR pathway in relation to tumourigenesis. Colussi et al. tested to what extent oxidative DNA damage played a role in the MMR mutator phenotype by expressing MTH1 in MSH2 deficient MEFs; the resulting decrease in DNA 8-oxoG levels translated into a decrease in the mutator effect [287]. Glaab et al. reported that growing the MLH1-deficient CRC cell line HCT116 in the antioxidant ascorbate, both with and without $\mathrm{H}_{2} \mathrm{O}_{2}$ treatment, significantly reduced mutation rates and reduced MSI by $30 \%$ [292]. Conversely, it has been suggested that MLH1-deficient, HCT116 cells are less sensitive to $\mathrm{H}_{2} \mathrm{O}_{2}$ than their MMR proficient counterparts (HCT116+Chr3) [292, 293]. This was attributed to an 
impaired apoptotic response in the HCT116 cells, suggesting that MMR is required for the recognition of severe oxidative damage and subsequent signalling to the apoptotic machinery. Our data suggest that $\mathrm{MSH} 2$ deficient cells are more sensitive to treatment with $\mathrm{H}_{2} \mathrm{O}_{2}$ [188]. It has recently been shown in a model for oxidative damage-induced tumours that loss of MSH2 significantly increased the formation of epithelial tumours in the small intestine [294]. Upon treatment with potassium bromate, Msh2 ${ }^{-/-}$mice displayed a 22.5-fold increase in tumour incidence [85, 294].

The evidence thus far suggests that the MMR system may suppress carcinogenesis in the context of oxidative damage by directly repairing ROS induced DNA lesions or acting as a sensor of oxidative damage and activating apoptosis

\subsection{Mitochondrial oxidative DNA damage repair}

\subsubsection{Repair of mitochondrial oxidative DNA damage by BER and NER}

Mitochondrial BER is similar to the nuclear pathway described above but there are some important differences that need to be noted. The majority of nuclear proteins involved in BER are soluble and after the activation of DNA damage response pathways they move to sub-nuclear foci. In the mitochondria the BER proteins are located in the inner mitochondrial membrane so DNA has to localise to this membrane to be repaired [295]. The BER proteins do not localise to the inner membrane in a DNA related fashion and studies have shown that the BER components are still present even in mitochondria with no DNA [295]. The four known glycosylases in the mitochondria that participate in BER are OGGI, NTHI, NEIL1 and NEIL2 [250]. Gap filling and DNA synthesis in the mitochondria is thought to be carried out by Pol $Y$ and more specifically Pol y1 for single nucleotides. This polymerase like Pol $\beta$ has dRP lyase activity and can change 5'dRP residues to 5'phosphate which are ligatable [296]. In mitochondrial-LP. BER it is thought that the nuclease helicase DNA2 stimulates the DNA synthesis activity of Pol y [248].

No definitive role for the NER pathways has been established in the mitochondria to date. A recent study by Pohjoismaki et al carried out genome wide transcriptional profiling examining cardiomyocyte development found an increase in the recruitment of 
DNA repair proteins including the NER proteins XPA and RAD23A in relation to increased oxidative damage during cardiac mitochondrial biogenesis [297].

\subsubsection{DNA break repair in the mitochondria}

DNA strand breaks occur in DNA as a consequence of endogenous and exogenous damage including oxidative DNA damage. Single strand breaks are known to be the result of oxidative damage to sugar moieties, erroneous BER repair and collision of topoisomerase I with RNA polymerases and DNA polymerases during transcription and replication. This form of damage in the nucleus is repaired by PARP and reports suggest that this repair protein in much smaller amounts, may be present in the mitochondria and forms a complex with mtDNA [298]. The proteins involved in SSB repair are similar to those involved in BER: APE1, PNKP and PolY [248]. Tyrosyl-DNA phosphodiesterase 1 (TDP1) is involved in the repair of SSBs formed during DNA replication and cells deficient in this enzyme have been found to have high levels of mitochondrial oxidative DNA damage [299]. Aprataxin which removes AMP from DNA has been shown to repair SSBs in the mitochondria which have formed as a result of abortive DNA ligase activity [300]

\subsubsection{Double strand break repair in the mitochondria}

There are more SSBs than double strand breaks DSBs as a consequence of single stranded DNA (ssDNA) being more vulnerable to attack. The nucleus has a complex network of three main pathways to repair DSBs: NHEJ, microhomolgy-mediated endjoining (MMEJ) and HR. The mitochondrial system for repair of DSBs is nowhere near as developed probably due to the fact that there are numerous copies of the genome in each mitochondria and therefore it is less imperative to repair all DSBs. Nevertheless, studies have shown that when DSBs are introduced into the mitochondrial genome they are most likely to be repaired by NHEJ [301]. Although the exact proteins involved in mitochondrial NHEJ are yet to be elucidated the evidence suggests that these are similar to the nuclear proteins involved in NHEJ [248]. For example, the expression of KU70 and KU86 have been detected in mitochondrial lysates and MRE11 has been found to co-localize with mtDNA [302]. It is thought that some form of HR takes place in the mitochondria but unlike the nuclear repair process where a second copy of the DNA 
is required for homology-dependent repair the evidence points to the fact that recombination between different mtDNA molecules is unlikely to take place [303, 304]. The link between deletions and re-arrangements of mitochondrial DNA and disease has now long been established. It is thought that a version of MMEJ is likely to be responsible for this given that there are often repeat sequences present at areas of deleted mitochondrial DNA [305].

\subsection{4 mtDNA degradation}

Mitochondrial DNA is more prone to attack than nuclear DNA especially by oxidative DNA damage so it is fortuitous that there are typically hundreds to thousands of copies of the mitochondrial genomes per cell. This means that if the DNA damage to mtDNA cannot be repaired, as is often the case with oxidative or alkylating damage, the DNA can be degraded without affecting mitochondrial function [248]. Shokolenko et al have shown that under high levels of oxidative stress the number of DNA strand breaks far outweighs the formation of oxidatively induced pre-mutagenic DNA lesions [306]. These strand breaks and abasic sites if left unrepaired lead to mtDNA degradation. It is thought that strand breaks are likely to occur as a consequence of stalled replication or transcription [306]. The most abundant nuclease in the mitochondria, Endonuclease G (EndoG) has been shown to carry out the degradation of mtDNA that cannot be repaired [307]. 


\subsection{The PGC1 family of co-activators}

\subsubsection{The role of the pgc1 family}

The PGC1- family of co-activators which are composed of three main members; PGC1$\alpha, P G C 1-\beta$ and PGC-related-co-activator (PRC). The first member to be discovered was PGC1- $\alpha$, which was found in brown fat for its role in interacting with the protein PPARY [308]. PGC1- $\beta$ has been identified to be most closely related to the first identified member of the PGC1- family, PGC1- $\alpha$ [309]. These two family members are similar in many key sequences including the $\mathrm{N}$-terminal activation domain, a C-terminal RNA binding domain and a central regulatory domain. PRC on the other hand does not share as many similarities to PGC1- $\alpha$ in its sequence structure compared to PGC1- $\beta$ [310].

The most pertinent characteristic of the PGC1- family is their ability to activate a wide range of transcription factors allowing them to carry out the role of 'master coregulators' of mitochondrial biogenesis. The PGC1- family are responsible for a key cellular function; alterations in metabolism through a diverse array of transcription factors as a result of changes in the environment [308]. Leone et al carried out a study investigating the phenotype that PGC1- $\alpha$ null mice exhibit and found a range of metabolic abnormalities including growth retardation of organs with high energy demands, high body fat, decreased numbers of mitochondria and respiratory capacity and a blunted thermogenic response [311].

\subsubsection{Mitochondrial biogenesis and the PGC1- family}

Mitochondrial biogenesis is a process, which has evolved to compensate for the depletion of damaged mitochondria. The term encompasses several functions: formation of the mitochondrial phospholipid double membrane [312], importing the large number of nuclear encoded mitochondrial proteins, translation of the mitochondrial encoded respiratory genes [313] and replication of mitochondrial DNA and translational machinery [314]. The PGC1- family achieves its role in mitochondrial biogenesis by interacting with a wide range of nuclear receptors (NRs) and transcription factors (TFs) to enable the expression of the mitochondrial proteome compromised of approximately 1500 proteins [315]. 
PGC1- $\alpha$ is known to be cold activated in brown muscle and fat and through activation of a range of transcription factors it induces mitochondrial biogenesis and uncoupled respiration leading to adaptive thermogenesis [308]. PGC1- $\beta$ on the other hand is not cold induced in brown fat and muscle [316] and has been found to share many but not all of its target genes with PGC1- $\alpha$. A study by Leone et al investigated differences in PGC1- $\alpha$ and PGC1- $\beta$ function using an adenoviral expression system in cell culture (hepatocytes) and in vivo [311]. The authors discovered that these two family members are similar in their metabolic function and both are able to induce a range of genes involved in oxidative metabolism but PGC1- $\beta$ did not induce genes involved in hepatic glucose metabolism. The authors concluded that this difference was partly due to the fact that PGC1- $\beta$ cannot interact with and co-activate hepatic nuclear receptor 4alpha (HNF4alpha) and FOXO1 [317]. St Pierre et al investigated the differences in mitochondrial respiration between cells expressing PGC1- $\alpha$ and those expressing PGC1- $\beta$ [318]. They showed that these two cell types are able to induce similar mitochondrial genes but PGC1- $\beta$ expressing cells had increased mRNA levels of glutamyl cysteine synthetase light and heavy subunit, both of which are known as activators of UCP2 and UCP3 which detoxify ROS [318]. Lin et al established from gain of function analysis that although PGC1- $\alpha$ and PGC1- $\beta$ are able to activate similar genes, PGC1- $\beta$ loss cannot completely compensate for PGC1- $\alpha$ loss [317]

Co-activators like the PGC1- members are multi-protein complexes which alter gene expression by allowing the downstream processes necessary for gene expression or repression. The most well known targets of the PGC1- family are the nuclear DNA binding transcription factors NRF1 and NRF-2alpha [319] which are discussed further in section 1.15.3. There are a multitude of TFs and NRs that have been found to interact with the PGC1- family to control mitochondrial biogenesis including the CAMP response element binding protein (CREB) [320] thyroid receptors [321], glucocorticoid receptors [322], estrogen related receptor (ERRa) [323], estradiol (ERa) [324] and peroxisome proliferator-activated receptor (PPARa and PPARY). Furthermore, some of the proteins involved in mitochondrial biogenesis are governed by a much wider group of extracellular and intracellular signalling pathways involving cellular lipid and carbohydrate metabolism, ROS detoxification and cell growth and differentiation [325]. 


\subsubsection{Mitochondrial biogenesis and NRF1 and NRF-2alpha}

The transcription of mitochondrial genes is mainly controlled by nucleo-mitochondrial interactions and the two main systems that are involved in this process are a few nucleus encoded transcription factors that bind directly to the mtDNA: transcription Factor A (Tfam), mitochondrial transcription Factor B (mtTFB) isoforms TFB1M and TFB2M) [326] and a set of transcription factors (including NRF1 and NRF-2alpha, stimulatory protein $1(\mathrm{Sp} 1)$, oestrogen related receptor $\alpha(E R R \alpha)$, and yin yang 1 transcription factor (YY1) that activate nuclear genes upstream that are required for respiratory chain expression [327, 328]. NRF1 and NRF-2alpha were first discovered through an effort to find TFs which could induce the respiratory genes cytochrome $\mathrm{c}$ and cytochrome oxidase [329, 330]. NRFI recognition sites were later identified in several promoter regions of genes involved in expression of respiratory chain complexes [331]. NRF1 has been identified as a positive regulator of transcription and binds as a homodimer to a palindromic recognition site in the cytochrome c promoter. Gugneja et al established that in mammalian cells NRF1 is a phosphoprotein and its phosphorylation at serine residues results in a conformational change in the NRF1 dimer enhancing its ability to bind DNA [332]. Several studies have established the key role that NRF1 plays in regulating respiratory chain complexes through both direct regulation of nuclear encoded subunits and indirectly to control the mitochondrial encoded subunits by regulating Tfam [333], tTFB and mitochondrial RNA polymerase [334]. The promoter regions of a large number of genes involved in mtDNA transcription and replication have been found to contain NRF1 and NRF-2alpha binding sites [335]. NRFI also controls several genes, which indirectly effect respiratory function through their genes products such as enzymes of the heme biosynthetic pathway [336], cytochrome $c$ assembly factor and members of the protein import (TTOM20, TOM70 and COX17) and assembly system (ribosomal proteins and tRNA synthetases) [327, 337]. NRF1 null mice display embryonic lethality and have been shown to have reduced levels of mtDNA and have deficiencies in maintaining a mitochondrial membrane potential [338]. Hoppeler et al have shown that exercise results in upregulation of PGC1- and NRF1 proving their role in regulating OXPHOS gene expression [339].

NRF-2alpha was later discovered through it specific binding in the cytochrome oxidase subunit IV (COXIV) promoter. The binding site for NRF-2alpha has been identified to 
contain the GGAA motif [335]. Experiments have revealed NRF-2alpha to have a role not only in COX subunit expression but also in the expression of all 10 cytochrome oxidase subunits encoded by nuclear DNA [340] and NRF-2alpha binding sites have been found in a multitude of genes related to the respiratory chain [335]. Furthermore, Verbasius et al have shown that both NRF1 and NRF-2alpha indirectly control mtDNA expression through activation of the mtTFA promoter TFB1M and TFB2M [341]. Glezer et al have shown that in fibroblasts that are stimulated with growth factors, the mRNA levels of three NRF target genes encoding mitochondrial transcription factors TFB1M, TFB2m and COXII are upregulated in preparation for increased growth [334]. NRF2alpha like NRF1 also has a role in driving the expression of proteins involved in protein import and assembly [342].

\subsubsection{Mitochondrial copy number}

Estimating the number of mtDNA molecules per cell is fraught with difficulty due to the fact that unlike the fixed copy number of the nuclear genome, there are numerous copies of the mitochondrial genome per cell [134]. Mitochondria are subject to fusion and fission leading to fluctuations in the number of mitochondrial genomes present within each mitochondria [343]. The most frequently used estimate to overcome this problem is measuring mtDNA copy number per cell. D'Erchia et al examined six different tissues from three different human individuals and found that there was a strong correlation between mtDNA copy number and mitochondrial gene expression levels, mitochondrial mass and respiratory function [344].

Hori et al have shown that ROS is involved in regulating mitochondrial copy number in yeast [345]. Holt et al propose that in mammalian DNA p53, ROS and the availability of the components necessary for biogenesis are all likely to play a role in deciding the fate of defective mitochondrial DNA. Several studies have shown that p53 associates with mtDNA and mtDNA binding proteins including POLG1 [346], TFAM (mtDNA maintenance protein) [347] and single stranded DNA binding protein [348]. Achanta et al have shown that the well known inhibitor of mitochondrial respiration Rotenone, causes increased ROS levels and promotes the translocation of p53 into the mitochondria [346]. Based on the studies described and the established role of p53 in deciding the fate of a cell with damaged nuclear DNA, Holt et al propose that in the 
mitochondria the role of p53 could be to decide whether the mtDNA should be retained or rejected under conditions of high ROS levels. It has been shown that when the levels of ROS are increased, p53 translocates to the mitochondria and that there is a significant decrease in mtDNA [349].

\subsection{Antioxidant system}

The antioxidant system is the essential first step in combating ROS and there are two main types of antioxidant: non-enzymatic and enzymatic. The non-enzymatic antioxidants include Vitamins $\mathrm{C}$ and $\mathrm{E}$, carotenoids, natural flavonoids, melatonin and thiol antioxidants (glutathione, thioredoxin and lipoic acid). The enzymatic antioxidant include superoxide dismutase (SOD), catalase and glutathione peroxidase. The gene expression of many of these antioxidants are controlled by several regulators including NF-E2-Related Factor 2 (NFE2L2 and also known as NRF2), FOXO and p53 [212] .

\subsubsection{NRF2 and the antioxidant defense system}

NRF2 (NFE2L2) is known as the master regulator of the antioxidant defense pathway. This key transcription factor detects signals activated due to cellular stress and activates the downstream genes necessary to respond to these signals. NRF2 is itself controlled by its inhibitor the Kelch-like ECH associated protein 1 (KEAP1)-Cullin 3 (CUL3) which ensures through binding with NRF2 that it remains inactive under normal physiological conditions [350]. This ensures that ROS levels are maintained at a level that allows for normal cell signalling but if ROS levels exceed a threshold then NRF2 can respond to counteract this. KEAP1 is composed of several cysteine residues and it is the 20 free sulphydryl (-SH) groups within this that carry out the role of sensing cell stress including oxidative stress [351]. ROS modify the cysteine residues such that a conformational change prohibits the degradation of NRF2 and allows its translocation to the nucleus [352]. This allows it to carry out its role as a transcription factor that controls over 100 genes involved in the antioxidant response including glutathione production and regeneration, NADPH, thioredoxins, quinones, catalase and enzymes involved in iron sequestration (Figure 1.6). NRF2 regulates these genes by binding to the antioxidant response elements (ARE) on DNA [352, 353]. 
The expression of the enzymes controlling the most abundant antioxidant, glutathione (GSH), is under the sole control of NRF2 [350]. GSH is readily available in the cytosol, nuclei and mitochondria. Glutamine cysteine ligase $(G C L)$ is responsible for driving the reaction between glutamate and cysteine. GCL is composed of two subunits the modifier (GCLM) and the catalytic subunit (GCLC) both of which are under the control of NRF2. The substrate which limits the production of GSH is cysteine and NRF2 also regulates the availability of this molecule through the expression of solute carrier family 7 member 11 (SLC7A11) which in turn drives the transcription of the cysteine/glutamate transporter XCT [354]. Furthermore, by governing the expression of several other antioxidant enzymes (GPX2, glutathione-s-transferases), NRF2 dictates the use of GSH since these enzymes detoxify ROS through GSH. This ROS detoxification results in the formation of the oxidised from of glutathione GSSG which is ultimately used to regenerate GSH using glutathione reductase and NADPH [203, 353] .

NADPH is produced by several metabolic pathways but NRF2 is able to modulate the expression of all NADPH-generating enzymes. NRF2 is also involved in the production and regeneration of thioredoxin and peroxiredoxins through controlling the expression of Thioredoxin 1 (TXN1) and peroxiredoxin 1 (PRDX1) as well as the fact that NADPH is required for the regeneration of thioredoxin via thioredoxin reductase (TXNRD) [203, 355].

The role of NRF2 in free $\mathrm{Fe}(\mathrm{II})$ homeostasis indirectly implicates it in ROS modulation through the Fenton reaction where $\mathrm{H}_{2} \mathrm{O}_{2}$ is converted to $\mathrm{OH} \cdot$ [356]. NRF2 encourages ROS detoxification because it controls the transcriptional upregulation of HMOX1[357] as well as the ferritin complex which deters the ROS producing fenton reaction taking place by converting $\mathrm{Fe}(\mathrm{II})$ to $\mathrm{Fe}(\mathrm{III})$ [203].

There are several other components apart from the main components of the antioxidant defense system mentioned above that are controlled by NRF2, as illustrated in Figure 1.6. 
Figure 1.6

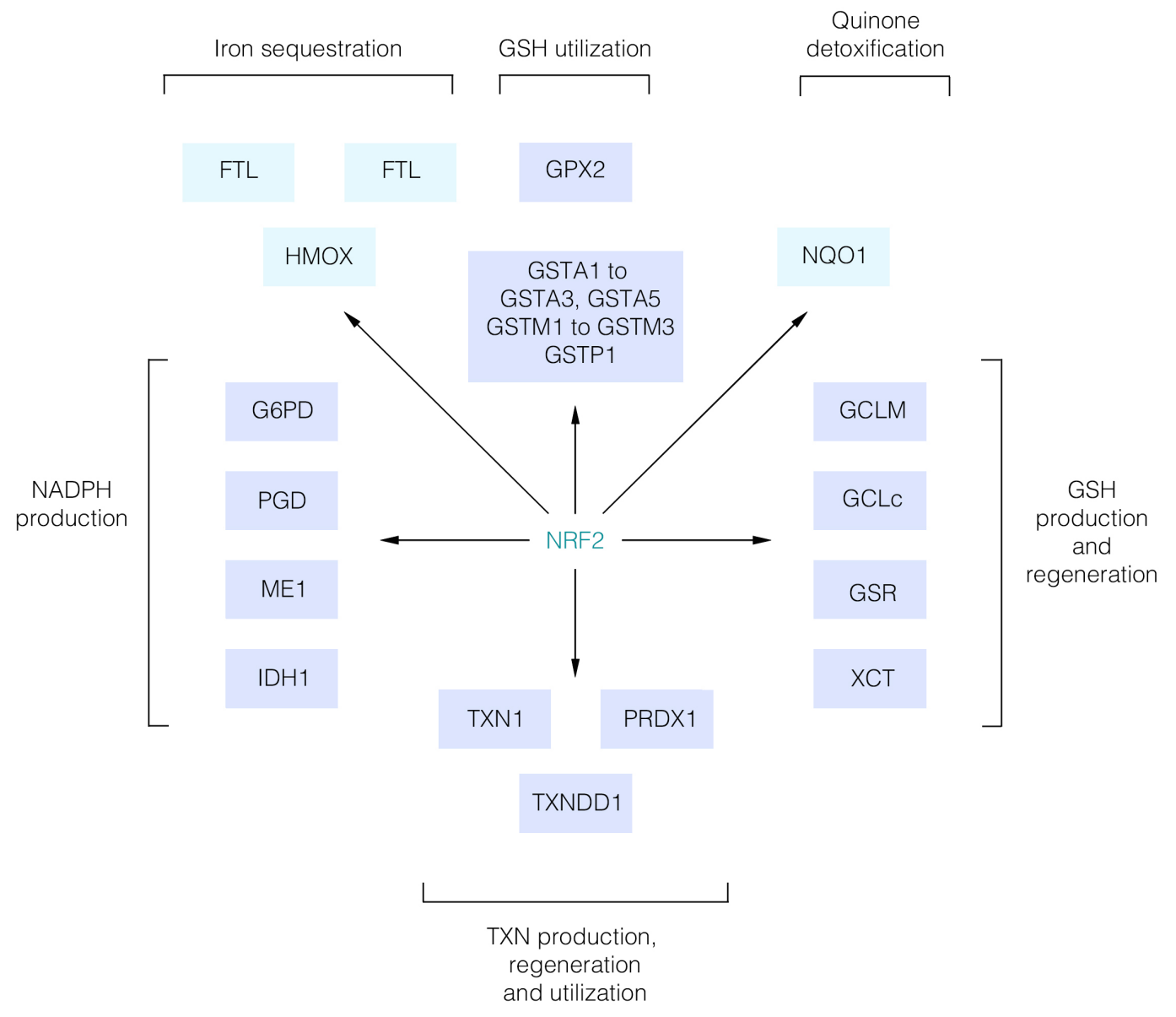


Figure 1.6. NRF2 regulates a multitude of antioxidant pathways.

Glutathione (GSH) synthesis and regeneration is regulated several enzymes under the control of NRF2: Glutamine cysteine ligase (GCL) (composed of glutamate-cysteine ligase complex modifier subunit (GCLM) and the GCL catalytic subunit (GCLC)) the cysteine/glutamate transporter XCT and glutathione reductase (GSR). GSH utilization is controlled by the glutathione S-transferases (GSTA1, GSTA2, GSTA3, GSTA5, GSTM1, GSTM2, GSTM3 and GSTP1) and glutathione peroxidase 2 (GPX2). Thioredoxin synthesis, regeneration and ultilization, are regulated by NRF2 through TXN1, thioredoxin reductase 1 (TXNRD1) and peroxiredoxin 1 (PRDX1). NADPH production is under the control of NRF, which controls the production of glucose-6-phosphate dehydrogenase (G6PD), phosphoglycerate dehydrogenase (PHGDH), malic enzyme 1 (ME1) and isocitrate dehydrogenase 1 (IDH1). GSH and TXN require NADPH to regenerate them once they have reduced ROS. NRF2 also controls $N A D(P) H: q u i n o n e$ oxidoreductase 1 (NQO1) and enzymes involved in haem iron sequestration, such as haem oxygenase. Adapted from Gorrini et al. (2013) Nat Rev [203] . 


\subsubsection{Antioxidant defense controlled by P53 and FOXO}

The tumour suppressor and forkhead box $\mathrm{O}$ (FOXO) family of transcription factors, mainly FOXO3a, have an essential role in controlling the expression of several antioxidant defense genes even though this is not one of their more well known roles. The main role of the FOXO transcription factors in the antioxidant pathway is in controlling the transcription of superoxide dismutases (SOD) [358], catalase and Sestrin 3 [359]. FOXO also plays a role in the control of several other antioxidants including glutathione, glutathione peroxidase and HMOX1 despite not directly controlling the transcription of these enzymes[203].

The tumour suppressor p53 is known to have both pro-oxdiant and antioxidant roles. Its pro-oxidant role relates to the fact that it promotes ROS production as a response to pathways resulting in apoptosis. The mechanism by which p53 acts as an antioxidant by increasing the transcription of NRF2, sestrin 2 , sestrin 3, GPx1. Furthermore, it also has two other target important targets; TP53-induced glycolysis and apoptosis regulator (TIGAR) and glutaminase 2 (GLS2). TIGAR encodes a protein that blocks glycolysis and encourages a shift towards pentose phosphate pathway mediated NADPH production. GLS2 is essential for GSH synthesis since it converts glutamine to glutamate and this leads to the production of GSH via GCLC and GCLM [203,360].

\subsubsection{Superoxide dismutases (SOD)}

The role of the superoxide dismutases is to convert the highly toxic $\mathrm{OH} \bullet$ to the less toxic $\mathrm{H}_{2} \mathrm{O}_{2}$ which can finally be reduced to $\mathrm{H}_{2} \mathrm{O}$ by catalase [361]. There are several different isoforms of SOD differing in several ways including the metal center and amino acids they are made from. The three human isoforms are the cytosolic form $\mathrm{Cu}, \mathrm{Zn}-\mathrm{SOD}$ (SOD1), the mitochondrial form Mn-SOD (SOD2) and the extracellular isoform EC-SOD [362]. This family of antioxidants carry out their function by utilizing the metal ion at their center to for successive oxidation and reduction reactions. Superoxide dismutase 1 which is composed of copper and zinc ions at its center carries out the dismutation of superoxide anions to hydrogen peroxide and water. Superoxide dismutase 2 is composed of one manganese atom per subunit and it undertakes a two-stage dismutation to detoxify ROS. This process results in the enzyme cycling between 
$\mathrm{Mn}(\mathrm{III})$ to $\mathrm{Mn}$ (II) and then back to Mn (III) [363]. EC-SOD does not directly detoxify ROS but is a glycoprotein which is under the regulatory control of cytokines [212].

\subsubsection{Catalase}

Catalase is one of the most efficient antioxidant enzymes being capable of detoxifying 6 million $\mathrm{H}_{2} \mathrm{O}_{2}$ into $\mathrm{H}_{2} \mathrm{O}$ and $\mathrm{O}_{2}$ each minute [212] :

\section{Catalase}

$2 \mathrm{H}_{2} \mathrm{O}_{2} \Longrightarrow 2 \mathrm{H}_{2} \mathrm{O}+\mathrm{H}_{2} \mathrm{O}$

\subsubsection{Glutathione peroxidase}

The glutathione peroxidase enzymes are responsible for counteracting low levels of oxidative stress. They are divided into two main forms: the selenium-independent form glutathione-s-transferase (GST) and the selenium-dependent GPx. The transcription factor FOXO also plays a role in glutathione metabolism although it is not one of its main functions, it controls the transcription of two of the gluthathione peroxidase family members, glutathione peroxidase 1 (GPX1) and glutathione-s-transferase (GSTM1). The GPx enzymes act as antioxidants by reducing peroxides to form selenoles (Se-OH) which have detoxifying properties because they stop peroxides from taking part in the Fenton reaction. GPx and glutathione metabolism is closely linked because it uses $\mathrm{H}_{2} \mathrm{O}_{2}$ or an organic peroxide $\mathrm{ROOH}$ and coverts this substrate to water (or alcohol) whilst oxidising glutathione at the same time. Catalase and GPx both use $\mathrm{H}_{2} \mathrm{O}_{2}$ as a substrate and compete for this molecule $[203,212]$.

\footnotetext{
GPx

$2 \mathrm{GSH}+\mathrm{H}_{2} \mathrm{O}_{2} \longrightarrow \mathrm{GSSG}+2 \mathrm{H}_{2} \mathrm{O}$
}

$2 \mathrm{GSH}+\mathrm{ROOH} \stackrel{\mathrm{GPx}}{\longrightarrow} \mathrm{GSSG}+\mathrm{ROH}+\mathrm{H}_{2} \mathrm{O}$ 


\subsubsection{The Sestrin family}

The sestrin family have been shown to exert their ROS detoxifying activity by restoring the activity of peroxiredoxins through their ability to reduce these enzymes when they have become over-oxidised. Sestrin 3 specifically is controlled by FOXO mediated transcription whereas sestrin 1 and sestrin 2 are controlled by p53 [364].

\subsubsection{The role of PGC1- family as antioxidants}

PGC1- $\alpha$ and PGC1- $\beta$ have been found to be activated by ROS induction and have been found to induce several antioxidant defense enzymes [318, 365, 366].The mechanism by which the PGC1 family controls these antioxidant has not been studied extensively but Kukidome et al have shown that PGC1- $\alpha$ decreases ROS levels and increases the mRNA levels of NRF1 and mitochondrial transcription factor A (mtTFA) [367]. St Pierre et al have recently demonstrated that when 10T1/2 cells are treated with $\mathrm{H}_{2} \mathrm{O}_{2}$ the expression of PGC1- $\alpha$ and PGC1- $\beta$ and a host of antioxidant genes (SOD1, SOD2, catalase, GPx1, ANT1, UCP2 and UCP3) are increased. This study found that the expression of PGC1- $\alpha$ was consistently much larger than the corresponding increase in PGC1- $\beta$ mRNA. The investigators also demonstrated that PGC1- $\alpha$ null mice were much more sensitive to oxidative stress and damage as indicated by increased 8oxoG staining in the brain cells of these mice compared to wildtype mice [365]. 


\section{Aims and objectives}

We have previously shown that MLH1 localises to the mitochondria [186] supported by a proteomic analysis of the mitochondria which identified MLH1 as a mitochondrial protein [368]. MLH1 deficiency has been found to be synthetically lethal with silencing of a number of mitochondrial genes $[186,187]$

Therefore the preliminary data strongly suggest that targeting mitochondrial function may be a potential therapeutic strategy for the treatment of MLH1-deficient tumours.

In addition there is a an unmet clinical need to try and identify targeted drugs to treat these groups of MLH1-deficient cancers, given that there is evidence to suggest that these tumours are resistant to the commonly used therapeutics. Bearing this in mind, the main aims for my project are as follows:

1, Validation of mitochondria-targeted compounds in a range of MLH1-deficient and proficient cell lines

2, Mechanistic analysis of mitochondrial function and compound selectivity in MLH1deficient cells. 


\section{Methods}

\subsection{Cell Culture}

\subsubsection{Cell lines}

The human colorectal isogenically-matched cell line HCT116 and HCT116+chr3 were a kind gift from Dr. A. Clark (NIEHS). The human ovarian cancer cell line SKOV3 were obtained from Cancer Research UK Cell Services (Clare Hall, South Mimms, Hertfordshire, UK) and the human ovarian cancer cell line IGROV were acquired from $\mathrm{NCl}-$ Frederick, MD, USA. The human ovarian cancer cell lines A2780 and A2780cp70 were a kind gift from Prof. Robert Brown (ICR, UK). The human colorectal cell lines SW620, HT29, SW480, SW48 and the human endometrial cell lines MFE296 and MFE280 cell lines were obtained from American Type Culture Collection (ATCC)

\subsubsection{Cell growth methods}

The cell stocks were made from early passage cells and stored in $10 \%$ Dimethyl sulfoxide (DMSO)/FBS in liquid nitrogen or at $-80^{\circ} \mathrm{C}$ for short-term storage. The HCT116, HCT116+chr3, HT29, A2780cp70, A2780, SW480, MFE-296, MFE-280 and SW48 cells were cultured in Dulbecco's modified eagle medium (DMEM) (Sigma) and the SKOV3 and IGROV cells were grown in the Rosewell Park Memorial Institute medium (RPMI 1640). The RPMI and DMEM media was supplemented with fetal bovine serum (FBS; Gibco $\AA$ Life Technologies, 10\% v/v), L-glutamine, sodium pyruvate, penicillin $(5 \mathrm{X} \mathrm{v} / \mathrm{v})$ and Streptomycin $(5 \mathrm{X} \mathrm{v} / \mathrm{v})$. Cell lines were maintained at $37^{\circ} \mathrm{C}$ in a humidified atmosphere with $5 \% \mathrm{CO}_{2}$ in T75 flasks (Corning). Cells were split 1:10-1:15 when confluency of $80 \%-90 \%$ was reached. The cells were washed with phosphate buffered saline (PBS) and then incubated for up to five minutes at $37^{\circ} \mathrm{C}$ with $5 \mathrm{mls}$ of $1 \mathrm{x}$ trypsin-EDTA (Gibco®, Life Technologies). Once the majority of cells had detached from the flask, the trypsin-EDTA was quenched by the addition of double the volume of media. Cells were collected and then pelleted by centrifugation for $3 \mathrm{~min}$ at $1200 \mathrm{rpm}$. Cells were then re-suspended in media and used for plating experiments (as described 
in section 3.1.3) and seeded in T75 flasks for further experiments. No cell line was cultured at passage higher than 10. All cell lines were authenticated on the basis of STR-profile, viability, morphologic inspection, and were routinely mycoplasma tested.

\subsubsection{Cell seeding conditions}

All experiments were performed in both biological and technical triplicate unless stated otherwise. Cells from flasks that were 80-90\% confluent and were seeded in either 96,12 or 6-well plates or $10 \mathrm{~cm}$ petri dishes (Corning) depending on the experiment. The cells were pelleted (as described in section 3.1.2) and re-suspended in fresh media. To establish the number of cells present per $\mathrm{ml}$ accurately, the cells were quantified using a Cell counter (TC20, BioRad) and plated at the appropriate density depending on cell type, the plate used and the experiment, as detailed throughout.

\subsection{Compounds}

All compounds were diluted in water or DMSO to a concentration of between $10 \mathrm{mM}$ $50 \mathrm{mM}$ and stored at between $4{ }^{\circ} \mathrm{C}$ and $-20^{\circ} \mathrm{C}$ according to manufacturer's instructions (Table 3.1) 
Table 3.1

\begin{tabular}{|c|c|c|}
\hline Compounds & Cat number & Company \\
\hline Antimycin A & SC202467A & Sigma-Aldrich \\
\hline Betulinic acid & CAS 472-15-1 & Abcam \\
\hline 3-Lapachone & SC200875A & Santa Cruz Biotechnology \\
\hline 2-Deoxy-D-Glucose & D8375 & Sigma-Aldrich \\
\hline Dichloroacetate & D6399 & Santa Cruz Biotechnology \\
\hline Fccp & SC203578 & Sigma-Aldrich \\
\hline Lonidamine & L4900 & Sigma-Aldrich \\
\hline Menadione & M9429 & Sigma-Aldrich \\
\hline N-Acetyl-Cysteine & SC202232A & Sigma-Aldrich \\
\hline Oligomycin & O5001 & Sigma-Aldrich \\
\hline Parthenolide & SC3523 & SC20342 \\
\hline Roteneone &
\end{tabular}

Table 3.1.Compounds used in this study and manufacturer's details

\subsection{Short term drug validation assays}

\subsubsection{Plating cells and drug treatment}

A panel of MLH1-deficient (SKOV3, IGROV, HCT116, A2780cp70, MFE-296) and proficient (HCT116+chr3, HT29, SW480, SW620, MFE-280) cell lines were plated at a density of between 1000-2000 cells/well in 96 well plates. The following day, cell medium was removed and replaced with either media containing vehicle (DMSO or water $(0.01 \%))$ or increasing concentrations of drug $(10 \mu \mathrm{M}-100 \mu \mathrm{M})$. Cell viability was estimated five days later using the CellTiter-Glo assay (Promega) according to the manufacturer's instructions (as described in section 3.3.2). The effect of each compound was determined by comparing luciferase readings from drug treated wells with those from wells treated with the compound vehicle to determine the surviving fraction. 


\subsubsection{Measuring cell viability}

Cell viability was measured using the ATP based luminescent CellTiter-Glo assay (Promega), which is based on the following reaction:

$$
\text { Beetle luciferin }+ \text { ATP }+02 \rightarrow \text { Oxyluciferin }
$$

The CellTiter-Glo reagent was thawed from $-20^{\circ} \mathrm{C}$ and diluted at a ratio of $1: 4$ with $1 \mathrm{X}$ PBS, prior to use. The diluted reagent $(100 \mu \mathrm{L})$ was added to each 96-well, after removing the drug containing media from the well. The 96-well plate was placed on a plate shaker (Grant-bio) and mixed for 2 minutes followed by an incubation period of 10 minutes at room temperature. Luminescence was estimated using a Wallac 1420 plate reader (PerkinElmer). To obtain the surviving fraction, the luminescence reading of each of the drug treated wells was normalized to the average luminescence reading of the control (vehicle-treated) wells.

\subsection{Protein analysis}

\subsubsection{Preparation of protein lysates}

For preparation of whole cell protein lysates, cells in 6 well plates were washed in 1XPBS and plates were then placed on ice, to prevent evaporation and reduce protein degradation. The cells were lysed in NP-40 lysis buffer $(20 \mathrm{mmol} / \mathrm{Tris}(\mathrm{pH}), 1 \mathrm{mmol} / \mathrm{l}$ EDTA, $0.5 \%(\mathrm{v} / \mathrm{v})$ NP40, 10\% (v/v) glycerol) and protease inhibitors (Roche) and kept on ice for 10 minutes. The lysed cells were then harvested using a cell scraper (BD Flacon, UK) and transferred to $1.5 \mathrm{ml}$ tubes and kept on ice for a further 15 minutes. The cells were then centrifuged to remove cell debris at $12,000 \mathrm{rpm}$ for 20 minutes at $4^{\circ} \mathrm{C}$ and the supernatant removed and either used fresh or stored at $-80^{\circ} \mathrm{C}$.

\subsubsection{Protein concentration determination}

The protein concentration was determined using Pierce BCA Protein Assay Kit (ThermoScientific) according to the manufacturer's instructions. A standard curve 
derived from the albumin stock provided in the kit was made by carrying out serial dilutions to give concentrations ranging from 125-2000 $\mu \mathrm{g} / \mathrm{ml}$. A sample of the dd $\mathrm{H} 2 \mathrm{O}$ used to produce these dilutions was also set aside to be kept with the standards. The standards were kept at $-20^{\circ} \mathrm{C}$. All standards and samples were plated out in duplicate in a 96-well plate. $10 \mu \mathrm{l}$ of double distilled water, standards and samples of unknown concentration were plated out in wells of a 96-well plate in duplicate. Reagent $A$ and Reagent B from the Pierce BCA Protein Assay Kit were then diluted at a ratio of 1:50 and $200 \mu \mathrm{l}$ of the mixture was added to each well containing either standard or sample using a multichannel pipette. The plate was placed on a plate shaker (Grant-Bio) for 1 minute, kept in the dark and incubated at $37^{\circ} \mathrm{C}$ for $30 \mathrm{~min}$. The absorption at $562 \mathrm{~nm}$ of each well was measured using the Wallac 1420 plate reader (PerkinElmer). A standard curve was plotted using the mean absorption readings for the standards. A scatter graph was plotted in Excel with protein concentration as the dependent variable on the $\mathrm{Y}$-axis and absorbance as the independent variable on the $\mathrm{X}$-axis. A polynomial trend line (2nd order regression) was added and the equation of the line was displayed, of the format $y=a x 2+b x+c$. The protein concentration was represented by $y$, therefore the equation and hence the protein concentration could be solved for each protein sample by entering the known mean absorbance value for that sample $(x)$.

\subsubsection{Western Blotting}

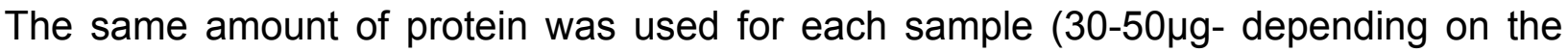
concentration of all the samples) and prepared with LDS loading buffer at a final concentration of $25 \mathrm{mM}$ DTT and incubated at $95^{\circ} \mathrm{C}$ for 5 minutes to denature the protein. The samples were then incubated on ice for $1 \mathrm{~min}$ and centrifuged at $12,000 \mathrm{rpm}$ for 30 seconds to ensure adequate mixing.

Protein lysates were eletrophoresed on 4-12\% Bis-Tris Novex precast gels (Invitrogen, UK) in 1X MOPS running buffer (Invitrogen). Molecular weight full-range rainbow markers (GE Healthcare Life) were loaded alongside the protein samples to allow size comparison of bands. Gels were run at $120 \mathrm{~V}$ for $90-120$ minutes. 
Proteins were transferred onto nitrocellulose membranes (GE Healthcare) at $20 \mathrm{~V}$ for 45 min using a semi-dry transfer apparatus (Bio-Rad) and transfer buffer. The membrane was equilibrated in transfer buffer $(9.6 \mathrm{mM}$ glycine, $1.2 \mathrm{mM}$ Tris base, $10 \%$ methanol in distilled water). All Extra ThickBlot Paper (Bio-Rad) used for the transfer was previously soaked in transfer buffer and the negative electrode of the transfer apparatus was wet with transfer buffer.

Following transfer, the membrane was blocked in blocking solution $(5 \%(\mathrm{w} / \mathrm{v}))$ milk powder in $0.1 \%$ PBS-Tween) for 1 hour at room temperature. The membrane was then incubated in primary antibody diluted in blocking buffer (normally 5\% milk powder unless the manufacturer's instructions specified) overnight at $4^{\circ} \mathrm{C}$ on a roller mixer. The primary antibodies used and the dilutions at which they were used are listed in Table 2.

The primary antibody was removed by washing the membrane three times with $0.1 \%$ PBS-Tween for 10 minutes, each wash. The membrane was then incubated with the appropriate horseradish peroxidase (HRP) conjugated secondary antibody (Table 3) diluted at a concentration of 1:5000 in 5\% milk and incubated for 1 hour at room temperature.

The membrane was then washed with three, 15 minute washes with $0.1 \%$ PBS-T as before. Protein expression was detected by chemiluminescence (SuperSignal West Pico Chemiluminescent substrate, Pierce, UK). Reagents 1 and 2 were mixed $1: 1$ and used to completely cover the membrane, followed by 5 minutes incubation at room temperature. Excess reagent was removed from the membrane and the resulting chemiluminescence signal was detected with Amersham Hyperfilm ${ }^{\mathrm{TM}}$ ECL (GE Healthcare). Immunoblotting for $\beta$-actin was used as a loading control. 
Table 3.2

\begin{tabular}{|c|c|c|c|c|}
\hline Antibody & Rasied in & Cat Number & Company & Concentration \\
\hline MLH1 & Rabbit & 4256 & Cell Signalling & $1: 1000$ \\
\hline MSH2 & Rabbit & sc-365052 & Santa Cruz & $1: 1000$ \\
\hline MSH6 & Rabbit & 3995 & Cell Signaling & $1: 1000$ \\
\hline NRF1 & Rabbit & 12381 & Cell Signaling & $1: 1000$ \\
\hline NRF2 & Rabbit & ab62352 & Abcam & $1: 1000$ \\
\hline Catalase & Rabbit & 12980 & Cell Signaling & $1: 5000$ \\
& & & & $1: 2000$ \\
\hline GPX1 & Rabbit & 3286 & Cell Signaling & \\
\hline NDUFB8 & Mouse & ab110242 & Abcam & $1: 1000$ \\
\hline Anti-Oxphos & Mouse & ab110411 & Novex & $1: 1000$ \\
\hline B-actin & & 5125 & Cell Signalling & $1: 10000$ \\
\hline
\end{tabular}

Table 3.2 : Primary antibodies and the concentration they were used

\section{Table 3.3}

\begin{tabular}{|c|c|c|c|}
\hline Antibody & Cat Number & Company & Concentration \\
\hline Anti-rabbit IgG & 7074 & Cell Signalling & $1: 5000$ \\
\hline Anti-mouse IgG & 7076 & Cell Signalling & $1: 5000$ \\
\hline
\end{tabular}

Table 3.3. Secondary antibodies and the concentration they were used 


\subsection{Measurement of complex I activity}

\subsubsection{Assay principle}

Complex I activity was measured using the Complex I activity ELISA assay (Abcam) The assay contains a pre-coated microplate with capture antibodies specific to complex I. Samples were prepared as in Section 3.5.2 and added to the pre-coated microplate wells. After the target has been immobilized in the well, Complex I activity is determined by following the oxidation of $\mathrm{NADH}$ to $\mathrm{NAD}+$ and the simultaneous reduction of the provided dye ( $\varepsilon=25.9 / \mathrm{mM} /$ well) which leads to increased absorbance at OD $450 \mathrm{~nm}$. The Complex I activity measured here is the NADH-dependent activity of Complex I which is not dependent on the presence of ubiquinone. This means that inhibitors, such as rotenone, which bind at or near the ubiquinone binding site do not inhibit this assay but assembly deficiencies of Complex I can affect this activity assay.

\subsubsection{Sample preparation}

Cells were grown in 6 well plates until they had reached $80-90 \%$ coluency and two wells were plated per cell line. A cell pellet was obtained as described in section 3.1.2 using $250 \mu$ l of trypsin per well. The final cell pellet for each cell line was re-suspended in $500 \mu \mathrm{l}$ of PBS. The protein was extracted from the cell by adding $10 \mathrm{X}$ non-ionic native detergent solution ( $\mathrm{n}$-dodecyl-b-d-maltoside Aka lauryl maltoside) provided in the kit (to a final dilution of 1/10) which fully lyses mitochondrial membranes (followed by incubating the samples on ice for 30 minutes. The samples was then centrifuged for 20 minutes at $4^{\circ} \mathrm{C}$ at $12,000 \mathrm{rpm}$. The supernatant was collected and protein concentration determined using the BCA assay described previously in section 3.4.2. The samples were diluted in the provided incubation solution (made by diluting 10X blocking solution with wash buffer) to a concentration of $200 \mu \mathrm{g} / 200 \mu \mathrm{l}$.

\subsubsection{Experimental procedure}

Equal amounts of protein $(200 \mu \mathrm{g})$ were loaded in the provided pre-coated 96 well microplate containing the enzyme NADH dehydrogenase. For the background wells, buffer only was added $(200 \mu \mathrm{l})$. The plate was then covered with foil and incubated at 
room temperature for 3 hours. The contents of the plate was then removed by turning the plate over onto a paper towel and blotting the plate face down onto it. To wash the plate, $300 \mu \mathrm{l}$ of $1 \mathrm{X}$ wash buffer was added to each well and removed by blotting on to a paper towel. This was step was repeated once more. The following assay solution was then prepared and the final volume made was dependent on the sample size.

\begin{tabular}{|c|c|c|c|}
\hline $\begin{array}{c}\text { No of strips (8 } \\
\text { well per strip) }\end{array}$ & 1X Buffer $(\mathrm{ml})$ & 20XNADH $(\mu \mathrm{l})$ & 100XDye $(\mu \mathrm{l})$ \\
\hline 1 & 1.57 & 84 & 17 \\
\hline
\end{tabular}

Two hundred microliters of the assay solution was added to each well and any bubbles formed in this process were popped immediately using a fine needle. The microplate was then placed on the plate reader (Wallac 1120) and the following program applied to measure the activity of Complex I over time by measuring the oxidation of NADH to $\mathrm{NAD}+$ and the simultaneous reduction of the dye, leading to increased absorbance at $450 \mathrm{~nm}$ over time.

Wavelength $450 \mathrm{~nm}$

Time 120 minutes

Interval between readings 1 minute

Temperature room temperature

Shaking Shake between readings

\subsection{Quantitative reverse transcriptase-polymerase chain reaction}

\section{(qRT-PCR)}

\subsubsection{RNA extraction and cDNA synthesis}

Before any RNA work was conducted, all surfaces, Eppendorf racks and pipettes were cleaned with RNaseZAP (Ambion) to remove any traces of RNase enzymes, which could contaminate samples and lead to RNA degradation. Total RNA was extracted from cells using the RNeasy Mini Kit (Qiagen), according to manufacturers instructions. 
Once the RNA extraction had been completed, the amount and purity of the RNA was determined by measuring absorbance at $260 \mathrm{~nm}$ (A260) and $280 \mathrm{~nm}$ (A280) with a NanoDrop-1000 Spectrophotometer. RNA absorbs light at $260 \mathrm{~nm}$, solvents at $230 \mathrm{~nm}$ and proteins at $280 \mathrm{~nm}$. A solvent contamination of our samples was observed by a ratio $260 / 230$ lower than 1.6. A protein contamination of our samples was observed by a ratio $260 / 280$ lower than 1.6 .

A volume with a total concentration of $1 \mu \mathrm{g}$ of RNA was used to synthesize cDNA using the Omniscript Reverse Transcriptase System for RT-PCR (Qiagen) with oligo dT primers, per the manufacturer's instructions.

\subsubsection{Experimental procedure}

Quantitiative RT-PCR (qRT-PCR) was performed using either SYBR Green (Applied Biosystems) or Taqman $®$ Assay-on-demand primer/probe assays (Applied Biosystems) and the primers used are listed below (Table 3.4). qRT-PCR was performed on the ABI Prism 7500 Sequence Detection system Instrument and software (PE Applied Biosystems). The qRT-PCR reactions for each biological repeat was carried out in triplicate and in duplicate for the patient samples due to a limited quantity of RNA. All qRT-PCR reactions were carried out in MicroAmp® Optical 96-Well Reaction Plates (Applied Biosystems, Life Technologies) sealed with MicroAmp® Optical Adhesive Films (Applied Biosystems, Life Technologies). The reaction conditions were as follows: $95^{\circ} \mathrm{C}$ for $10 \mathrm{~min}$ to activate the DNA polymerase, followed by 40 cycles of $95^{\circ} \mathrm{C}$ for 15 sec and $60^{\circ} \mathrm{C}$ for $1 \mathrm{~min}$. Fluorescence readings were taken at the end of the $60^{\circ} \mathrm{C}$ incubation. All qRT-PCR experiments contained negative controls consisting of the mastermix and nuclease free water instead of cDNA.

\subsubsection{1 $\operatorname{TaqMan}^{\circledR}$ Gene Expression Assay}

qRT-PCR was carried out using the TaqMan® Universal PCR Master Mix (PE Applied Biosystems) .

Each well contained the following reagents to make up a total volume of $20 \mu$ :

10 $\mu$ TaqMan® Universal PCR Master Mix 
$7 \mu \mathrm{l} \quad$ DEPC $\mathrm{H}_{2} \mathrm{O}$

$1 \mu \mathrm{l}$ Sample cDNA

$1 \mu \mathrm{l} \quad$ Probe/primer assay for gene of interest

$1 \mu \mathrm{l} \quad$ Probe/primer assay for $\beta$-actin

The cycling program consisted of $10 \mathrm{~min}$ at $95^{\circ} \mathrm{C}$ followed by 40 cycles of $95^{\circ} \mathrm{C}$ for 15 sec and $1 \mathrm{~min}$ at $60^{\circ} \mathrm{C}$.

\subsubsection{SYBR Green}

The primers used for qRT-PCR with SYBR Green (NDUFA and TUBB) were a kind gift from Professor Gyorgy Szabadkai's laboratory (Department of Cell and Developmental Biology, UCLH) and The primers were used at a concentration of $0.15 \mu \mathrm{M}$

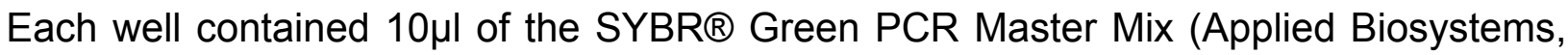
Life technologies), forward and reverse primers (volume dependant on concentration used) and nuclease free water was added to make up a final reaction volume of $20 \mu$.

The reaction conditions were as outlined above in section 3.6.2 but for primer optimization, an additional dissociation step was added at the end of the PCR program which consisted of $95^{\circ} \mathrm{C}$ for $15 \mathrm{sec}, 60^{\circ} \mathrm{C}$ for $20 \mathrm{sec}$, a $19 \mathrm{~min} 59 \mathrm{sec}$ ramping step and then $95^{\circ} \mathrm{C}$ for $15 \mathrm{sec}$. The dissociation data was collected on the ramping step. 
Table 3.4

\begin{tabular}{|c|c|}
\hline Gene Symbol & Cat Number \\
\hline$\beta$-ACTIN & NM_001101.2 \\
\hline PGC1A (PPARGC1A) & Hs01016719_m1 \\
\hline PGC1B (PPARGC1B) & Hs00991677_m1 \\
\hline NRF1 & Hs00192316_m1 \\
\hline NRF2 (NFE2L2) & Hs00975961_g1 \\
\hline ANT & Hs00154037_m1 \\
\hline Catalase & Hs00156308_m1 \\
\hline Gpx1 & Hs00829989_gH \\
\hline SOD1 & Hs00533490_m1 \\
\hline SOD2 & Hs00167309_m1 \\
\hline MLH1 & Hs000179866_m1 \\
\hline & \\
\hline & \\
\hline & \\
\hline & \\
\hline & \\
\hline & \\
\hline & \\
\hline & \\
\hline
\end{tabular}

Table 3.4. Taqman ${ }^{\circledR}$ Primers used for qRT-PCR experiments

\subsubsection{Analysis}

Target mRNA was normalised $(\Delta \mathrm{CT})$ to $\beta$-actin by subtracting the cycle threshold (Ct) of the $\beta$-actin sample from the cycle threshold $(\mathrm{Ct})$ of each target gene of interest for each sample. The expression level of each target gene was determined relative to the MLH1 proficient cell line or in the case of the patient samples the cDNA from normal surrounding MMR proficient tissue.

$\Delta \Delta \mathrm{CT}=\Delta \mathrm{CT} \mathrm{MLH} 1$ deficient $-\Delta \mathrm{CT} \mathrm{MLH} 1$ proficient

This was expressed as a fold change in gene expression using the formula: 
Fold change $=2^{-\Delta \Delta C T}$

\subsection{Measurement of oxidative consumption rate (OCR) and spare respiratory capacity}

\subsubsection{Day 1: cell plating}

Oxidative consumption rate $(\mathrm{OCR})$ and spare respiratory capacity (SPR) were measured using the Seahorse extracellular flux analyser ( $\mathrm{XF}^{\mathrm{e}} 24$; Seahorse Bioscience). Cells were plated in a 24 well plate designed for use with the $\mathrm{XF}^{\mathrm{e}} 24$ in appropriate media as in section 3.3.1. The number of cells plated was calculated with the aim to achieve $80-90 \%$ confluency the following day and $100 \mu \mathrm{L}$ of cell suspension was used per well. No cells were plated in the designated temperature control wells as shown in grey in the plate layout below (Figure 3.1) Cells were grown overnight $\left(37^{\circ} \mathrm{C}, 5 \% \mathrm{CO}_{2}\right)$. 


\section{Figure 3.1}

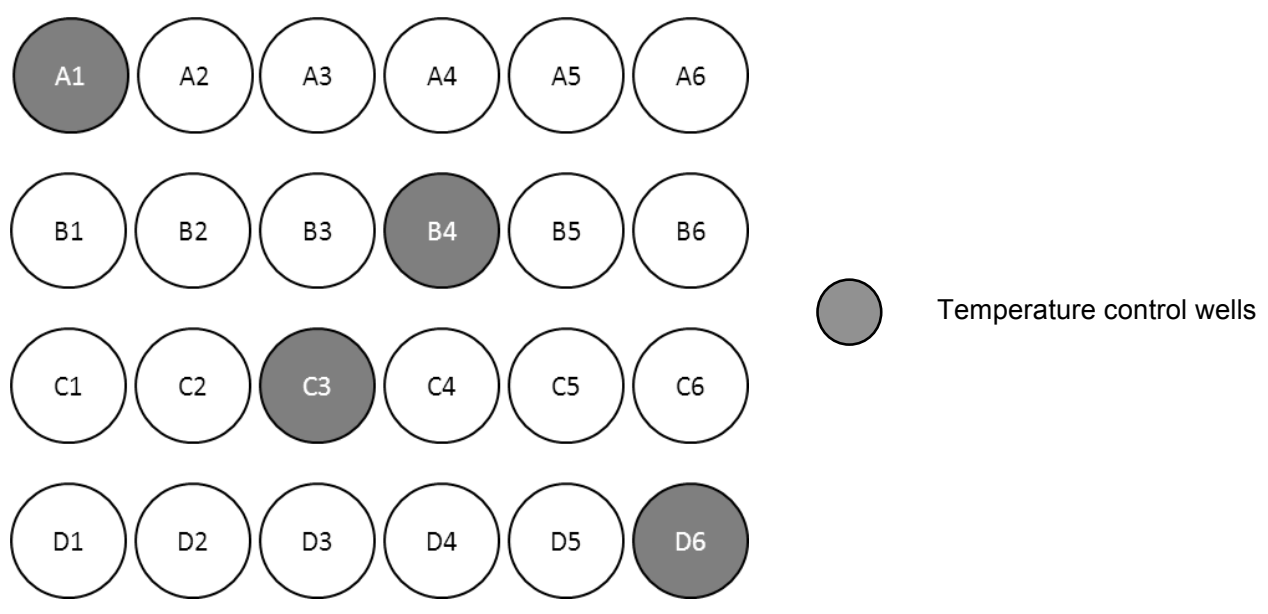

Figure 3.1: Layout of cell culture plate for $\mathrm{XF}^{\mathrm{e}} 24$

\subsubsection{Day 1: Preparing calibration plate}

Calibration plates were obtained specifically designed for use with the $\mathrm{XF}^{\mathrm{e}} 24$ and one plate was required for every cell culture plate used. The calibration plate is composed of two seperate parts: a sensor cartridge with ports to allow addition of drugs which is placed on top of a 24 well calibration plate (Figure 3.2). The sensor cartridge was hydrated by adding $1 \mathrm{ml}$ of $\mathrm{XF}^{\mathrm{e}} 24$ calibrant solution (Seahorse Bioscience) to each well of the calibrant plates. The plate was wrapped in parafilm to prevent evaporation and incubated overnight $\left(37^{\circ} \mathrm{C}\right.$ and $\left.0 \% \mathrm{CO}_{2}\right)$. 


\section{Figure 3.2}

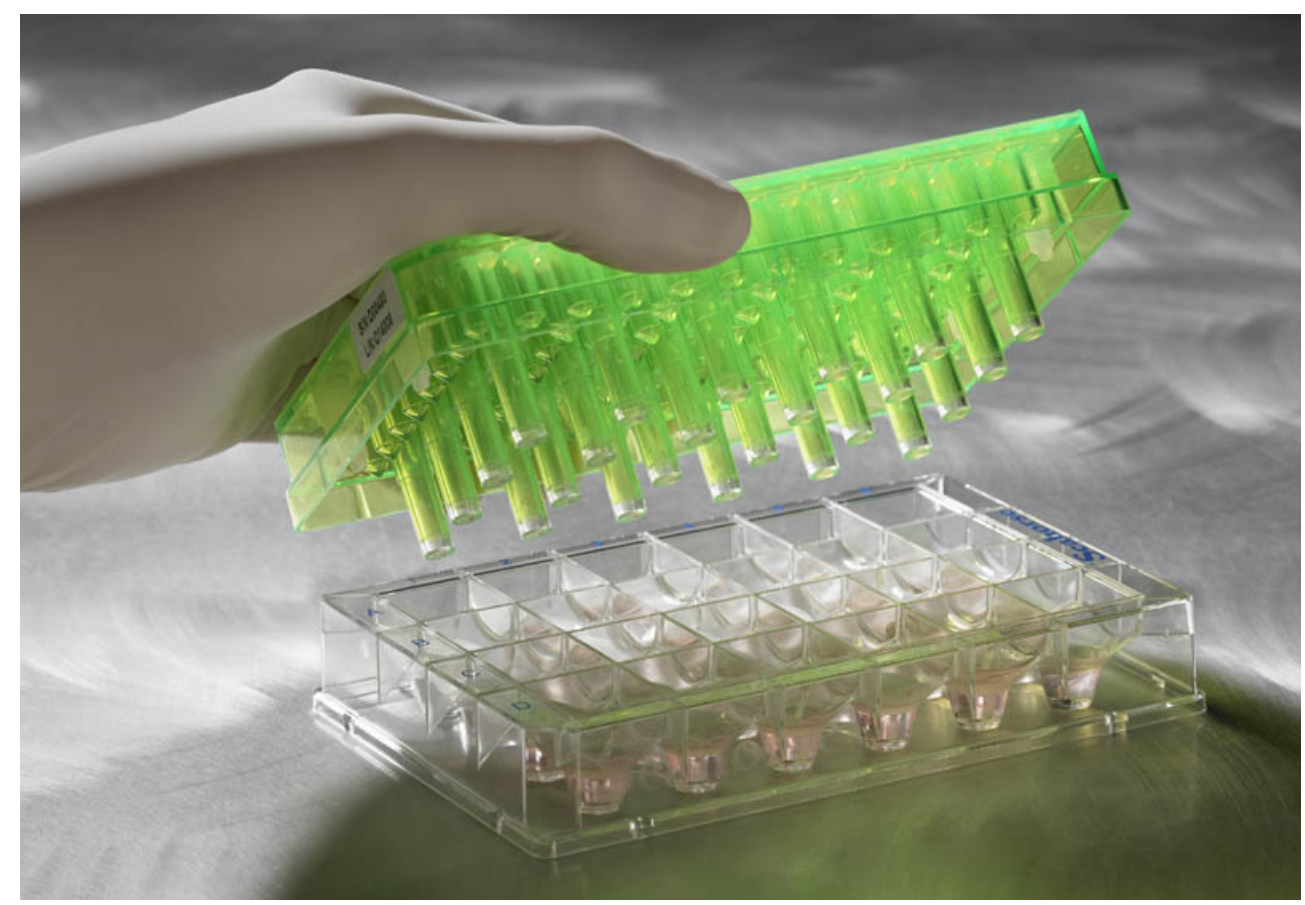

Figure 3.2: Illustration of calibration plate with sensor cartridge containing drug ports in the top plate. Taken from How to run an XF assay. Taken from Agilent XF Seahorse Technology user manual(2016) [369].

\subsubsection{Day 2: preparing the cell culture plate}

The media from the $\mathrm{XF}^{\mathrm{e}} 24$ cell culture plate was removed and replaced with $500 \mu \mathrm{L}$ for each well (including temperature control wells) of XF assay media (Seahorse Bioscience, 102365-100) supplemented with L-glutamine (5\% v/v), sodium pyruvate $(5 \% \mathrm{v} / \mathrm{v})$, penicillin $(5 \mathrm{X} \mathrm{v} / \mathrm{v})$ \& streptomycin $(5 \mathrm{X} \mathrm{v} / \mathrm{v})$, glucose $4.5 \mathrm{gr} / \mathrm{L}$ and the $\mathrm{pH}$ of the media was adjusted to 7.4 . The cell culture plate was incubated at $37^{\circ} \mathrm{C}$ in a non- $\mathrm{CO}_{2}$ incubator for at least 60 minutes.

\subsubsection{Day 2: preparing and running the calibration plate}

The ports in the sensor cartridge (labelled A-D) were loaded with the indicated drugs (Table 3.5 ) at $10 \mathrm{X}$ concentration so that the final desired concentration would be achieved once the drug mixes with the $500 \mu$ l of XF assay media that is in each well of the cell culture plate. Figure 3.3 below details what the addition of each drug measures, 
to achieve a full bioenergetic profile. The calibration plate with the loaded sensor cartridge on top is placed in the $\mathrm{XF}^{\mathrm{e}} 24$ machine and the automated calibration programme is run. 
Table 3.5

\begin{tabular}{|c|c|c|c|c|}
\hline Drug & $\begin{array}{c}\text { Mechanism } \\
\text { of action }\end{array}$ & Measurement & $\begin{array}{l}\text { Initial volume } \\
\text { and } \\
\text { concentration } \\
\text { injected }\end{array}$ & $\begin{array}{c}\text { Final } \\
\text { concentration }\end{array}$ \\
\hline $\begin{array}{c}\text { Oligomycin } \\
\text { (port A) }\end{array}$ & $\begin{array}{c}\text { Inhibits } \\
\text { Complex V }\end{array}$ & Proton leak & $55 \mu \mathrm{l}$ of $10 \mu \mathrm{M}$ & $1 \mu \mathrm{M}$ \\
\hline $\begin{array}{l}\text { FCCP } \\
\text { (Port B) }\end{array}$ & $\begin{array}{l}\text { Uncoupling } \\
\text { agent }\end{array}$ & $\begin{array}{c}\text { Spare } \\
\text { respiratory } \\
\text { capacity }\end{array}$ & $60 \mu \mathrm{l}$ of $0.75 \mu \mathrm{M}$ & $0.075 u m$ \\
\hline $\begin{array}{l}\text { Antimycin A } \\
\text { (port C+D) }\end{array}$ & $\begin{array}{c}\text { Inhibits } \\
\text { complex III }\end{array}$ & $\begin{array}{l}\text { Non- } \\
\text { mitochondrial } \\
\text { respiration }\end{array}$ & $\begin{array}{c}65 \mu \text { of } 10 \mu \mathrm{M} \\
(\text { port } \mathrm{C}) \\
70 \mu \text { l of } 10 \mu \mathrm{M} \\
(\text { port } \mathrm{D})\end{array}$ & $\begin{array}{l}1 \mu \mathrm{M} \\
1 \mu \mathrm{M}\end{array}$ \\
\hline $\begin{array}{l}\text { Rotenone } \\
\text { (port C+D) }\end{array}$ & $\begin{array}{c}\text { Inhibits } \\
\text { Complex I }\end{array}$ & $\begin{array}{l}\text { Non- } \\
\text { mitochondrial } \\
\text { respiration }\end{array}$ & $\begin{array}{c}65 \mu \text { of } 10 \mu \mathrm{M} \\
(\text { port } \mathrm{C}) \\
70 \mu \text { l of } 10 \mu \mathrm{M} \\
(\text { port } \mathrm{D})\end{array}$ & $\begin{array}{l}1 \mu \mathrm{M} \\
1 \mu \mathrm{M}\end{array}$ \\
\hline
\end{tabular}

Table 3.5. Drugs used for XF analyzer. 


\section{Figure 3.3}

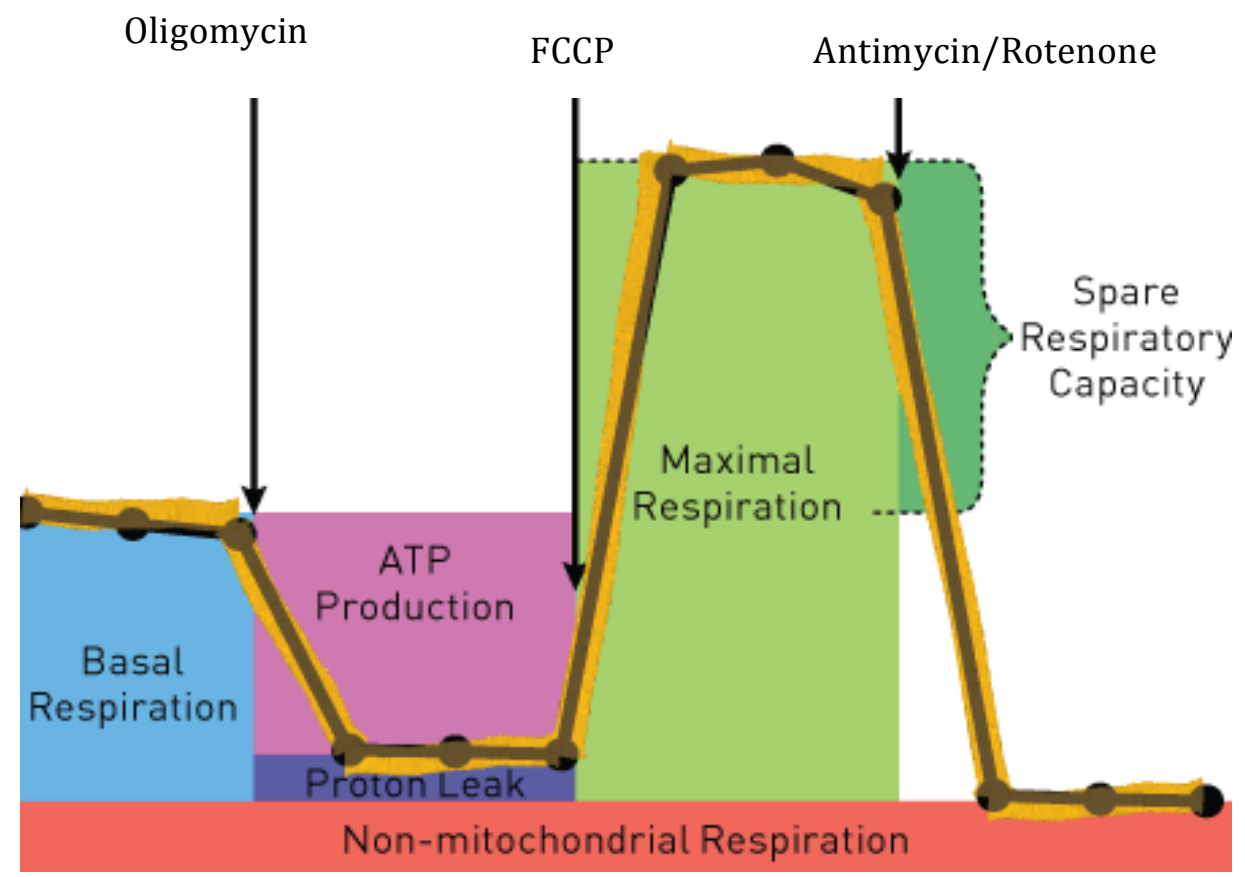

Figure 3.3. Bioenergetic profile. Adapted from How to run an XF assay. Taken from Agilent XF Seahorse Technology user manual(2016 )[369].

\subsubsection{Running the cell culture plate}

The cell culture plate is loaded immediately after optimal calibration has occurred and the calibration plate is ejected. The machine retains the sensor cartridge with the loaded drugs and the cell culture plate is then inserted and the machine places the sensor cartridge on top of the cell culture plate so that the appropriate drugs can be added. The following programme was then set up and run to determine the OCR: 
Further Calibration and Mixing $15 \mathrm{~min}$

Measure

Mix

Wait

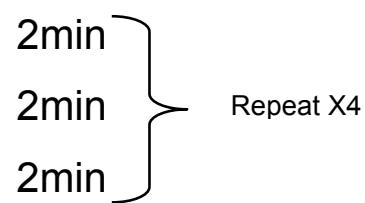

Measure baseline

$2 \mathrm{~min}$

Mix

Wait

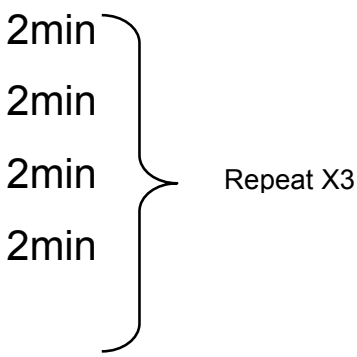

Mix

Wait

$2 \mathrm{~min}$

$2 \mathrm{~min}$

Inject (Port B)

Measure

$2 \mathrm{~min}$

Repeat X3

Mix

Wait

Inject (Port C)

Measure

Mix

Wait

Inject (Port D)

Measure
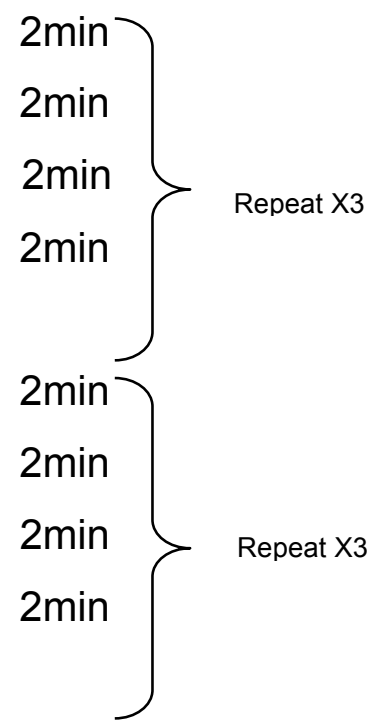

The spare respiratory capacity was calculated by taking away the basal OCR (measured prior to addition of any drugs) from the maximal respiration (measured after FCCP injection) 


\subsection{Measuring ROS using the MitoXpress}

Cellular ROS levels were measured using the MitoXpress (Luxcel Biosciences) instrument, which allows high throughput fluorescence microscopy. The cells were plated in a 96 well black/clear bottom plate (BD Falcon) in DMEM or RPMI as described in section 3.3.1. The following day, media was removed and replaced with media containing either DMSO, Antimycin (positive control, $1 \mu \mathrm{M}, 10 \mu \mathrm{M}$ and $100 \mu \mathrm{M}$ ) or Parthenolide $(10 \mu \mathrm{M})$ and incubated for 30 minutes at $5 \% \mathrm{CO}_{2}$. After this 30 minute incubation the drug-containing media was aspirated and $100 \mu \mathrm{L}$ of media containing the

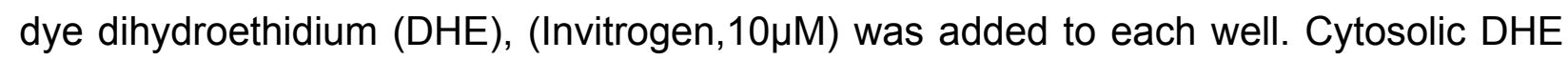
exhibits blue fluorescence but once it reacts with superoxide anions it becomes oxidized to 2-hydroxyethidium, it intercalates with DNA, and stains the cell with a red fluorescence (ExcitationEmission 530/380 (nm)) [370]. The fluorescence was measured in the same number of sites and cells per well by the MitoXpress instrument. Each experiment was carried out in triplicate. The data was analysed by Zhi Zhao (Department of Cell and Developmental Biology, UCLH) by obtaining the fluorescence values from the MitoXpress instrument measured at 530nm (W3) and 380nm (W3) for each sample and calculating a ratio between these values and then taking the average increase rate of the ratio. 
3.9 Sequencing the mitochondrial genome using the Illumina ${ }^{\circledR}$ Miseq $^{\mathrm{TM}}$ platform

The mitochondrial genome of the HCT116 and HCT116+chr3 cell lines was sequenced on the Illumina ${ } \mathrm{MiSeq}^{\mathrm{TM}}$ platform as per the manufacturers guidelines [371]. The main steps are outlined in the diagram below (figure 3.4) and briefly described. 


\section{Figure 3.4}

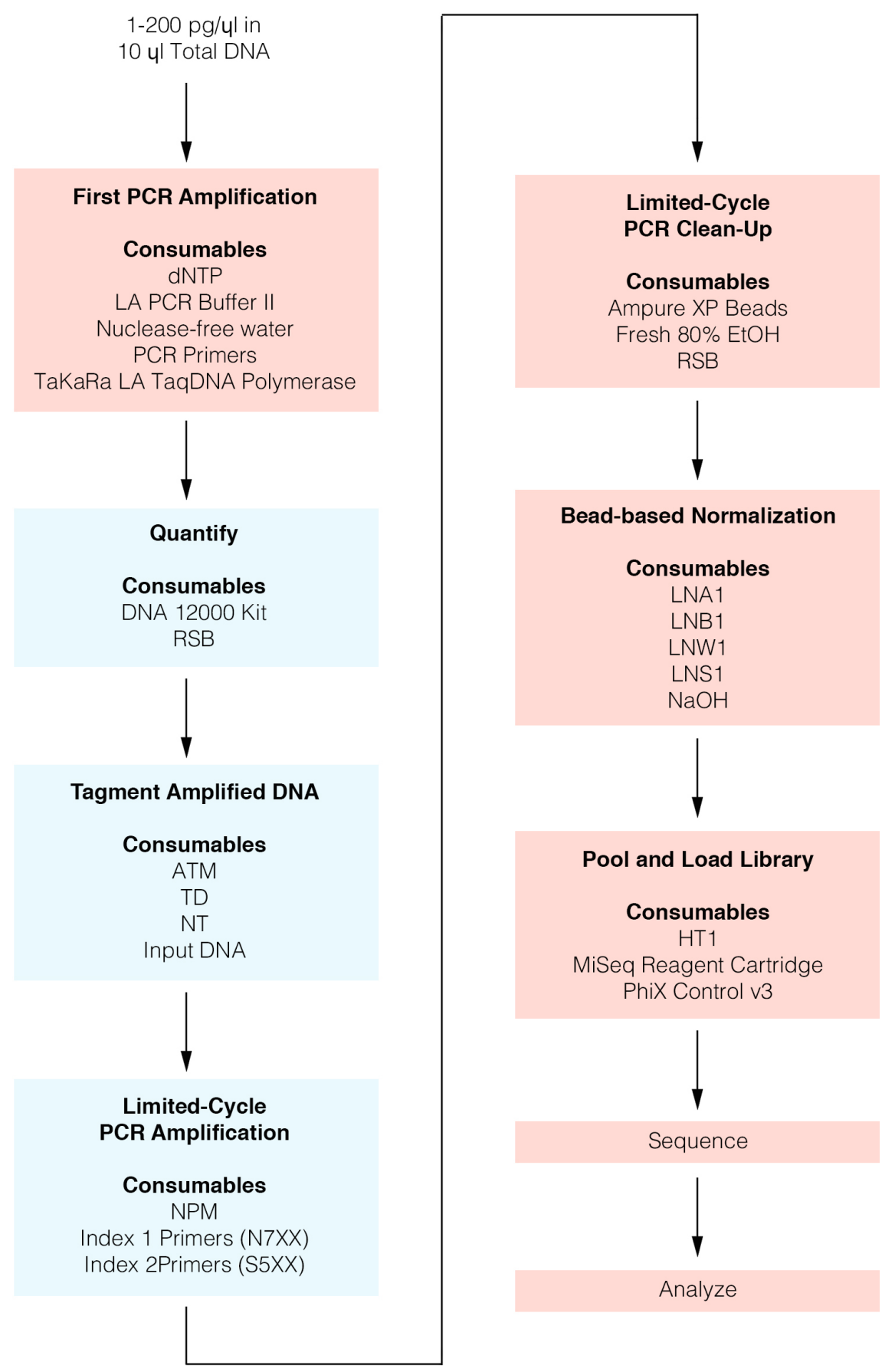

Figure 3.4. Schematic of main steps for mtDNA sequencing using Illumina the Platform. Adapted from Ilumina. Human mtDNA Genome Guide (2016) [371] 


\subsubsection{Sample preparation}

The HCT116 and HCT116+chr3 cell lines were grown in T75 flasks and when cells reached $80-90 \%$ confluency, they were trypsinised as in section 3.1.2. One million $\left(1 \times 10^{6}\right)$ cells were counted for each cell line and pelleted. Three biological repeats were used for each cell line for this experiment and the experiment was carried out in duplicate for each repeat. Genomic DNA was extracted from these cell pellets using the using the QIAmp DNA Mini Kit (Qiagen). The concentration and quality of DNA samples were measured using the ND-1000 NanoDrop as described for RNA quantification in section 3.6.1.

\subsubsection{Target enrichment}

The aim of this step was to obtain two long overlapping PCR amplicons spanning the entire human mtDNA genome as described in the TakaRa LA Taq PCR kit (TaKaRa). The forward and reverse primers used were MTL1 and MTL2 (Table 3.6). The amount of template DNA used was 1.0ng per reaction. The volume of reagents and total PCR reaction volume used was half of that described in the manufacturers protocol $(20 \mu l$ total volume). The PCR master mix outlined below (Table 3.7) and DNA were plated in a PCR plate and sealed. 
Table 3.6

\begin{tabular}{|c|c|}
\hline Primer & Sequence \\
\hline MTL-F1 & $\begin{array}{c}5^{\prime} \text { - AAA GCA CAT ACC AAG GCC AC } \\
-33^{\prime}\end{array}$ \\
\hline MTL- F2 & $\begin{array}{c}\text { 5'- TAT CCG CCA TCC CAT ACA TT - } \\
3 \text {, }\end{array}$ \\
\hline MTL- R1 & $\begin{array}{c}\text { 5'- TTG GCT CTC CTT GCA AAG TT } \\
-3^{\prime}\end{array}$ \\
\hline MTL-R2 & $\begin{array}{c}\text { 5'- AAT GTT GAG CCG TAG ATG CC } \\
-3,\end{array}$ \\
\hline
\end{tabular}

Table 3.6. Primers used for mtDNA sequencing 


\section{Table 3.7}

\begin{tabular}{|c|c|}
\hline Reagent & Volume ( $\mu \mathrm{l})$ \\
\hline $2.5 \mathrm{mM}$ dNTP mix & 1 \\
\hline 10X LA PCR Buffer II (25 mM MgCl2) & 2.5 \\
\hline $\begin{array}{c}\text { Diluted Forward Primer (e.g., MTL-F1 } \\
\text { or MTL-F2) }\end{array}$ & 1 \\
\hline $\begin{array}{c}\text { Diluted Reverse Primer (e.g., MTL-R1 } \\
\text { or MTL-R2) }\end{array}$ & 1 \\
\hline Nuclease-free water & 11.25 \\
\hline 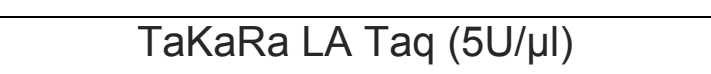 & 0.25 \\
\hline Total & 20 \\
\hline
\end{tabular}

Table 3.7. Reagents used for first PCR amplification and volumes per well

Amplification was performed on a thermal cycler (Bio-Rad, Tetrad 2) machine using the following parameters:

$94^{\circ} \mathrm{C}$ for 5 minutes

30 cycles of:

$--98^{\circ} \mathrm{C}$ for 15 seconds

-- $68^{\circ} \mathrm{C}$ for 10 seconds (slow ramp from $68^{\circ} \mathrm{C}$ to $60^{\circ} \mathrm{C}$ at $0.2^{\circ} \mathrm{C}$ per second)

-- $60^{\circ} \mathrm{C}$ for 15 seconds

-- $68^{\circ} \mathrm{C}$ for 11 minutes

$72^{\circ} \mathrm{C}$ for 10 minutes

Hold at $10^{\circ} \mathrm{C}$

The amplified product was held at $10^{\circ} \mathrm{C}$ overnight and at $4^{\circ} \mathrm{C}$ until it was required. The following day, the DNA products were checked for the correct size and quantified for subsequent normalisation to $0.2 \mathrm{ng} / \mu \mathrm{L}$ using a 2200 TapeStation instrument (Agilent, Stockport, UK). The MTL-F1 and MTL-R1 primer pairs generated $9065 \mathrm{bp}$ and a single peak in the range of 7705-10425bp. The MTL-F2 and MTL2-R2 primer pair generated 11170 bp and a single peak in the range of 9495-12845 bp. Each PCR amplicon was 
diluted in RSB buffer (Nextera XT DNA Sample Preparation Kit, Illumina) to a final concentration of $0.2 \mathrm{ng} / \mu \mathrm{l}$ and the entire volume was transferred to a PCR plate.

\subsubsection{Tagment amplified DNA}

The next step tags and fragments the input DNA using the human mtDNA genome sample prep transposome (Nextera XT DNA Sample Preparation Kit, Illumina) which both fragments the DNA and adds adapter sequences to the ends to allow amplification by PCR. The following reagents were used for this step to make the Nextera XT Tagment Amplicon (NTA) plate .

\section{Nextera XT DNA Sample Preparation Kit reagents:}

ATM (Amplicon Tagment Mix)

TD (Tagment DNA Buffer)

NT (Neutralize Tagment Buffer)

Input DNA (0.2 ng/ $\mu \mathrm{l}) 5 \mu \mathrm{l}$ per sample (1 $\mathrm{ng}$ total)

After thawing the reagents the following mix was prepared in a PCR plate per well.

\section{Table 3.8}

\begin{tabular}{|c|c|}
\hline \multirow{2}{*}{ Reagent } & Volume $(\mu \mathrm{l})$ \\
\hline TD Buffer & 10 \\
\hline Input DNA $(0.2 \mathrm{ng} / \mu \mathrm{L})$ & 5 \\
\hline ATM & 5 \\
\hline
\end{tabular}

Table 3.8 Reagents used for DNA tagmentation and volumes per well

After centrifuging the plate to $280 \times \mathrm{g}$ at $20^{\circ} \mathrm{C}$ for 1 minute the plate is placed in a thermal cycler (Bio-Rad, Tetrad 2) and the following program applied: 
$55^{\circ} \mathrm{C}$ for 5 minutes

Hold at $10^{\circ} \mathrm{C}$

The samples were neutralized by adding $5 \mu \mathrm{l}$ of NT Buffer to each well of the NTA plate. The plate was centrifuged at $280 \mathrm{Xg}$ at $20^{\circ} \mathrm{C}$ for 1 minute and then placed at room temperature for 5 minutes.

\subsubsection{Limited-Cycle PCR Amplification}

The rationale behind this step was to amplify the tagmented DNA. In addition this step also adds the index 1 (i7) and index 2 (i5) primers, which are required for cluster formation.

The following reagents were required for this step

\section{Nextera XT DNA Sample Preparation Kit contents:}

NPM (Nextera PCR Master Mix)

Index 1 primers (N7XX) 1 tube each index -

Index 2 primers (S5XX) 1 tube each index

TruSeq Index Plate Fixture 1

After the above reagents were thawed and mixed the primers were arranged as below in the TruSeq Index Plate Fixture (Figure 3.6) 


\section{Figure 3.6}

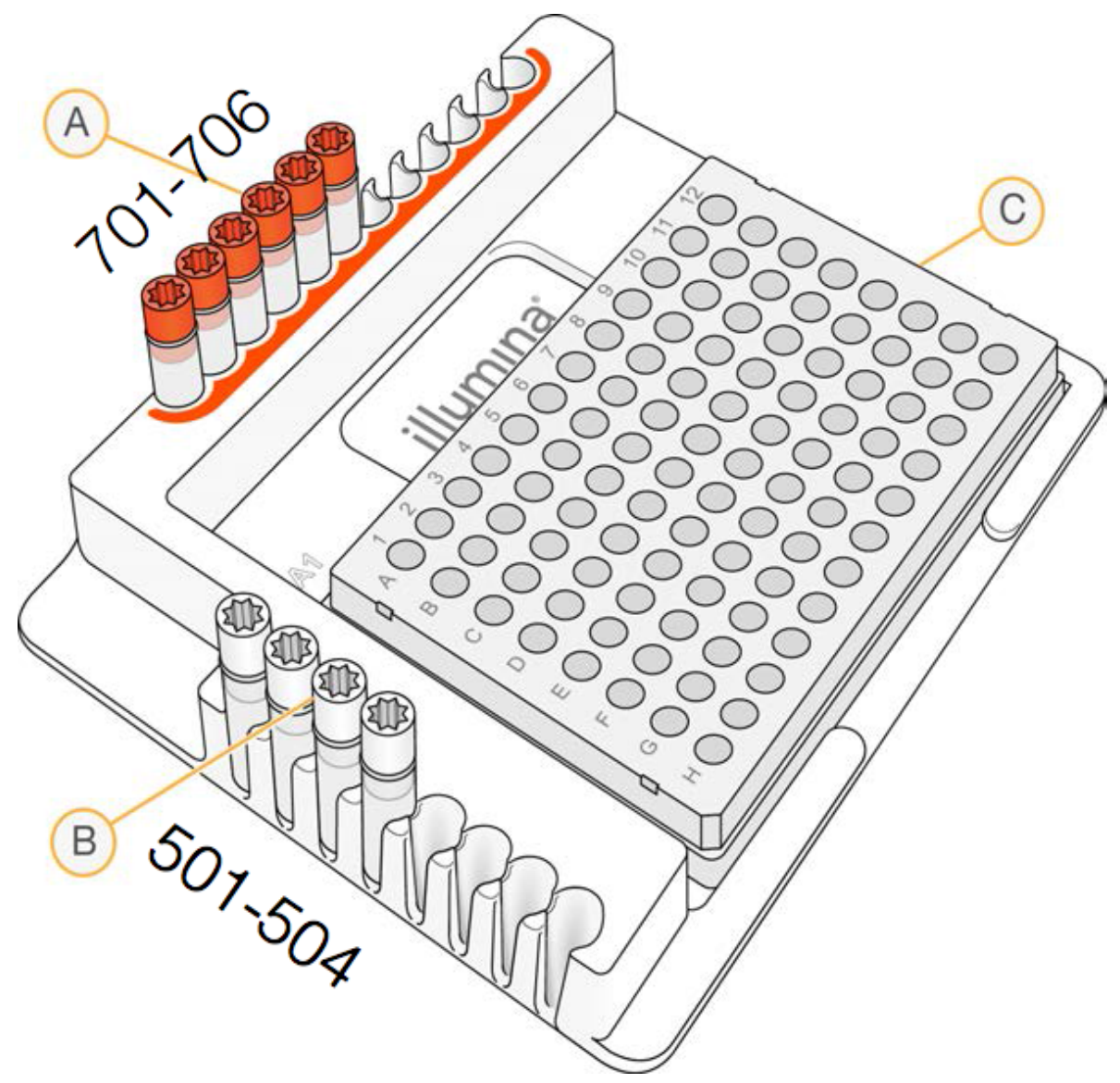

$$
\begin{aligned}
& A=\text { Index Primer } 1 \text { (i7) (Orange Caps) } \\
& B=\text { Index Primer } 2 \text { (i5) (White Caps) } \\
& C=\text { NTA plate }
\end{aligned}
$$

Figure 3.6: TruSeq index plate with arrangement of index 1 and 2 primers displayed. Taken from llumina. Human mtDNA Genome Guide (2016) [371] .

The dual indexing approach uses two 8 base indices: Index 1 (i7) and Index 2 (i5) (Table 3.9). These indices allow unique indexing of samples by adding one adapter from Index 1 (N701-N706) and one adapter from Index 2 (S501-S504). 
Table 3.9

\begin{tabular}{|c|c|c|c|}
\hline Index 1 (i7) & Sequence & Index 2 (i5) & Sequence \\
\hline N701 & TAGATCGC & S501 & TAGATCGC \\
\hline N702 & CGTACTAG & S502 & CTCTCTAT \\
\hline N703 & AGGCAGAA & S503 & TATCCTCT \\
\hline N704 & TCCTGAGC & S504 & AGAGTAGA \\
\hline N705 & GGACTCCT & & \\
\hline N706 & TAGGCATG & & \\
\hline
\end{tabular}

Table 3.9. Sequence of index 1 and 2 primers.

The NTA plate was placed in the TruSeq plate fixture and the following reagents are added to each well (Table 3.10). The primers were added in different combinations as shown in the diagram below (Table 3.11)

Table 3.10

\begin{tabular}{|c|c|}
\hline Reagent & Volume $(\boldsymbol{\mu l})$ \\
\hline NPM & 15 \\
\hline Index 2 primers (White Cap) & 5 \\
\hline Index 1 primers (Orange cap) & 5 \\
\hline
\end{tabular}

Table 3.10 Reagents used for limited cycle PCR-amplification and volumes per well. 
Table 3.11

\begin{tabular}{|l|l|l|l|l|l|}
\hline 116 1 & Chr3 1 & 116 2 & Chr3 2 & 116 3 & Chr3 3 \\
MTL1A & MTL1A & MTL1A & MTL1A & MTL1A & MTL1A \\
N701/S501 & N702/S501 & N703/S501 & N704/S501 & N705/S501 & N706/S501 \\
\hline 116 1 & Chr3 1 & 1162 & Chr3 2 & 1163 & Chr3 3 \\
MTL1B & MTL1B & MTL1B & MTL1B & MTL1B & MTL1B \\
N701/S502 & N702/S502 & N703/S502 & N704/S502 & N705/S502 & N706/S501 \\
\hline 1161 & Chr3 1 & 1162 & Chr3 2 & 1163 & Chr3 3 \\
MTL1A & MTL1A & MTL1A & MTL1A & MTL1A & MTL1A \\
N701/S503 & N702/S503 & N703/S503 & N704/S503 & N705/S503 & N706/S503 \\
\hline 1161 & Chr3 1 & 116 2 & Chr3 2 & 116 3 & Chr3 3 \\
MTL1B & MTL1B & MTL1B & MTL1B & MTL1B & MTL1B \\
N701/S504 & N702/S504 & N703/S504 & N704/S504 & N705/S504 & N706/S504 \\
\hline
\end{tabular}

Table 3.11: Unique indexing used for samples in this experiment.

The plate was then sealed and centrifuged at $280 \times \mathrm{g}$ at $20^{\circ} \mathrm{C}$ for 1 minute. The plate was then placed on a thermal cycler (Bio-Rad, Tetrad 2) and the following program applied:

$95^{\circ} \mathrm{C}$ for 30 seconds

12 cycles of:

$95^{\circ} \mathrm{C}$ for 10 seconds

$-55^{\circ} \mathrm{C}$ for 30 seconds

$-72^{\circ} \mathrm{C}$ for 30 seconds

$72^{\circ} \mathrm{C}$ for 5 minutes

Hold at $10^{\circ} \mathrm{C}$

\subsubsection{Limited-Cycle PCR clean-up}

This step purifies the library DNA using AMPure XP beads and removes very short library fragments from the population. The following reagents were used for this step

AMPure XP beads

$80 \%$ Ethanol 
RSB (Nextera XT DNA Sample Preparation Kit contents)

The library DNA was purified by adding $30 \mu$ l of mixed AMPure beads to each well of the NTA plate, which contained 50ul PCR amplified library. The plate was then subsequently placed on a magnetic stand and the supernatant discarded. Two ETOH washes were then carried out on the magnetic stand as per manufacturers instructions. The NTA plate was then left to air dry on the magnetic stand and a pellet obtained, which was re-suspended in $52.5 \mu \mathrm{l}$ of RSB and then placed on the magnetic stand. The supernatant from each well of the NTA plate was transferred to the corresponding well of a plate named LNP (Library Normalization Plate).

\subsubsection{Bead Based Normalization}

This step aims to normalize the quantity of each library so that there is an even distribution of each library in the pooled sample.

The following reagents are required for this step

Nextera XT DNA Sample

Preparation Kit contents:

LNA1 (Library Normalization Additives)

LNB1 (Library Normalization Beads 1)

LNW1 (Library Normalization Wash 1)

LNS1 (Library Normalization Storage Buffer 1)

$0.1 \mathrm{~N} \mathrm{NaOH}$

After all the reagents were thawed and LNA1 was mixed with LNB1 45ul of the LNA1/LNB1 was added to each well of the LNP plate (see section 3.9.5), which contains sample and libraries. The plate was sealed and placed on a microplate shaker at $1800 \mathrm{rpm}$ for 30 minutes. The LNP plate was then placed on a magnetic stand and $80 \mu \mathrm{l}$ of supernatant removed. The plate was then removed from the magnetic stand and the beads washed with LNW1 twice, as per manufacturers instructions. 
A SGP (StoraGe Plate) barcode label was applied to a PCR plate and this was now known as the SGP plate.

The LNP plate was again removed from the magnetic stand and $30 \mu$ of $0.1 \mathrm{~N} \mathrm{NaOH}$ added to each well to elute the sample. The plate was sealed and placed on a microplate shaker at $1800 \mathrm{rpm}$ for 5 minutes. LNS1 (30 $\mu \mathrm{l})$ was added to each well to be used in the SGP plate. After the 5 minute elution, the LNP plate was again placed on the magnetic stand for 2 minutes or until the liquid was clear. The supernatant form the LNP plate $(30 \mu \mathrm{l})$ was then transferred to the SGP plate.

\subsubsection{Pool and load from bead-based normalization}

This part of the method prepares for cluster generation and sequencing, equal volumes of the bead based normalized library is combined, diluted in hybridization buffer and heat denatured for Miseq sequencing

The following reagents are require for this step

\section{Nextera XT DNA Sample}

Preparation Kit contents:

HT1 (Hybridization buffer)

MiSeq reagent cartridge

PhiX Control v3

An eppendorf tube was labeled PAL (Pooled Amplicon Library) and a second, DAL (Diluted Amplicon Library). All reagents were thawed. From the SCP plate $5 \mu$ l of each library to be sequenced was transferred column by column, to a PCR eight-tube strip. PhiX control $(5 \% 2 \mathrm{~nm})$ was added to the library in the eight tube strip.

The contents of the PCR tubes were combined and transferred to the tube labeled PAL. Following this, 576ul of HT1 was transferred to the DAL tube and then 24ul of the PAL tube was transferred to the DAL tube that contains HTI. The DAL tube was then incubated at $96^{\circ} \mathrm{C}$ for $2 \mathrm{~min}$ and then kept on ice. The DAL was loaded into a thawed 
miSeq reagent catridge in the load samples reservoir. The SGP plate was sealed and ready to proceed to the library sequencing on the Illumina ${ }^{\circledR}$ Miseq $^{\mathrm{TM}}$

\subsection{8 sequencing on the Illumina ${ }^{\circledR}$ Miseq $^{\mathrm{TM}}$}

Sequencing reactions were carried out using the MiSeq v2 (150bp) chemistries (Illumina) according to the manufacturer's recommendations. The MiSeq re-sequencing protocol for small genome sequencing was followed according to the manufacturer's recommendations. On-board software (i.e., Real-TimeAnalysis and MiSeq Reporter) converted raw data to Binary Alignment/Map (BAM) and Variant Call Format (VCF) v4.1 files using Genome Analysis Toolkit (GATK). During this process, the sequenced region of interest (ROI) was aligned to the revised Cambridge Reference Sequence (rCRS). Each nucleotide position ( $\mathrm{np}$ ) was interrogated and variations from the reference were annotated by base difference.

\subsubsection{Analysis}

Analysis was carried out by Dr. Jun Wang (Department of Bioinformatics, Dr Claude Chalala's lab) using the MiSeq Reporter software and Variant Analyzer software.

\subsection{Measuring mitochondrial copy number changes}

The protocol for this experiment is adapted from and has been previously described [372].

\subsubsection{Creating standards}

$\beta 2$-microglobulin and mitochondrial tRNA forward and reverse primers were designed and the sequences are described below (Table 11). Plasmids containing the $\beta 2-$ microglobulin $(\beta 2 \mathrm{M})$ gene and mitochondrial tRNA gene were a kind gift from Professor Chris Boschoff's lab (UCLH). The plasmid concentrations were determined using the Nanodrop. These were then linearized by digesting the plasmids using the BAMH1 (New England Biolabs) restriction enzyme as detailed in the manufacturers instructions. The DNA digestions were cleaned up using a PCR-Clean Up kit (Qiagen) as per the 
manufacturers instructions. The linearized plasmids were quantified using the Nanodrop and the following equation was used to calculate the copies/ $\mu \mathrm{l}$ :

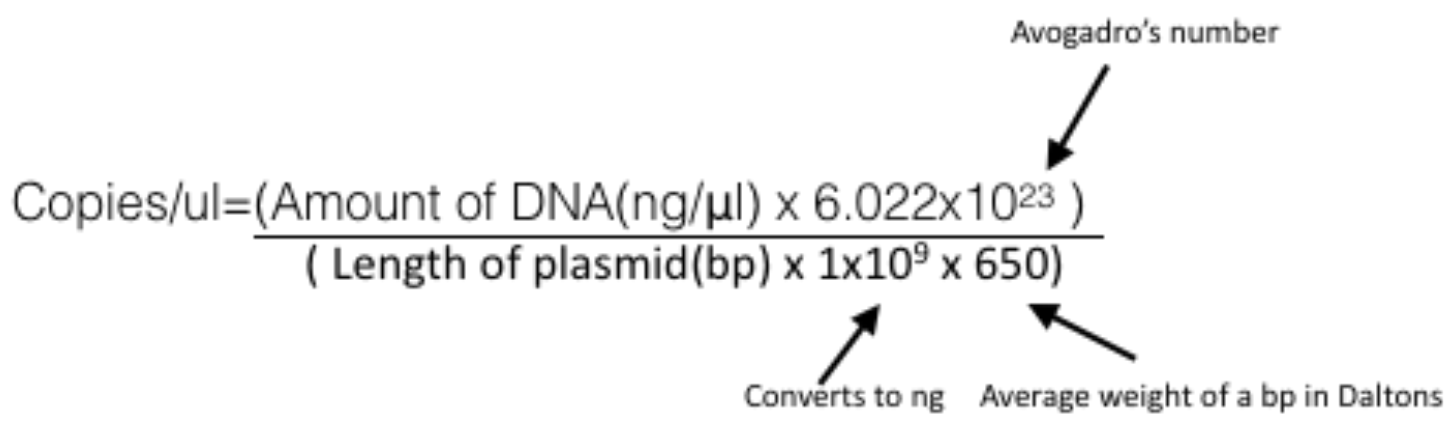

The $\beta 2 \mathrm{M}$ plasmid is known to be $4000 \mathrm{bp}$ long and the tRNA plasmid is $3650 \mathrm{bp}$ long.

For example, for the $\beta 2 \mathrm{M}$ linearized plasmid:

Copies $/ \mu \mathrm{l}=\left[5.1(\mathrm{ng} / \mu \mathrm{l}) \times 6.022 \times 10^{23}\right]=1181238462 \mathrm{copies} / \mu \mathrm{l}$ $\left[4000(\mathrm{bp}) \times 1 \times 10^{9} \times 650\right.$ 
Table 3.12

\begin{tabular}{|c|c|}
\hline Primer & Sequence \\
\hline$\beta 2$-microglobulin-F & TGCTGTCTCCATGTTTGATGTATCT \\
\hline$\beta 2$-microglobulin-R & TCTCTGCTCCCCACCTCTAAGT \\
\hline Mitochondrial tRNA-F & CACCCAAGAACAGGGTTTGT \\
\hline Mitochondrial tRNA-R & TGGCCATGGGTATGTTGTTA \\
\hline
\end{tabular}

Table 3.12. Sequences of plasmids used to quantify mitochondrial copy number.

\subsubsection{Harvesting genomic DNA}

A cell pellet was obtained by the method described in section 3.3.2 and either frozen down at $80^{\circ} \mathrm{C}$ for later use or DNA extracted immediately. The DNA was extracted using the Qiagen DNeasy Blood and Tissue DNA extraction kit protocol and quantified using a Nanodrop. DNA samples were then diluted to $1 \mathrm{ng} / \mathrm{\mu l}$ using clean $\mathrm{ddH}_{2} \mathrm{O}$.

\subsubsection{Preparing qPCR plate}

The $\beta 2 \mathrm{M}$ and tRNA primers were diluted $1 / 10$ in $\mathrm{ddH}_{2} \mathrm{O}$ to give $10 \mu \mathrm{M}$ working solutions. The following qPCR master mix per well was prepared, one each for $\beta 2 \mathrm{M}$ and tRNA (Table 3.13) 
Table 3.13

\begin{tabular}{|c|c|}
\hline Reagent & Volume $(\boldsymbol{\mu l})$ \\
\hline Sybr Green & 6.5 \\
\hline $\mathrm{ddH}_{2} \mathrm{O}$ & 12.5 \\
\hline Forward primer $(10 \mu \mathrm{M})$ & 2.5 \\
\hline Reverse primer $(10 \mu \mathrm{M})$ & 2.5 \\
\hline
\end{tabular}

Table 3.13. Reagent used and volumes per well for qPCR to ascertain mitochondrial copy number.

To each well of an Optical 96-Well Reaction Plate, $24 \mu$ l of the master mix and $1 \mu$ l of the relevant standard or diluted genomic DNA was added. Each biological repeat of the samples and the standards was carried out in triplicate. The reaction conditions were as follows:

$95^{\circ} \mathrm{C}$ for $10 \mathrm{~min}$

40 cycles of

$--95^{\circ} \mathrm{C}$ for $15 \mathrm{sec}$ and

$--60^{\circ} \mathrm{C}$ for $1 \mathrm{~min}$.

Fluorescence readings were taken at the end of the $60^{\circ} \mathrm{C}$ incubation.

To calculate the exact number of mitochondrial copies in our sample, the $\mathrm{Ct}$ values were plotted against the known number of copies per standard dilution and analysing by logarithmic regression.

\subsubsection{Analysis}

Standard curves of both tRNA and $\beta 2 \mathrm{M}$ were generated by plotting the mean $\mathrm{Ct}$ value of the standards against concentration of the standard. A logarithmic regression was performed on excel and the resulting equation was used to calculate the copy number in the samples by applying the CT values for these genes in the samples to the 
equation generated (as below). The standard curves were only used if the efficiency was $\geq 99 \%$.

$\mathrm{Ct}=\mathrm{m}^{*} \ln (\mathrm{x})+\mathrm{b}$, where

$\mathrm{M}=$ slope,

$\mathrm{b}=\mathrm{y}$ intercept and

$\mathrm{x}=$ sample's genome copy number

This allowed determination of the log copy number and $10^{\wedge} X$ (where $X$ is the log copy number) determined the actual number of nuclear (for $\beta 2 \mathrm{~m}$ ) and mitochondrial genomes (tRNA). For $\beta 2 \mathrm{M}$, the nuclear copy number/cell was calculated by dividing the copy number by two. Finally the mitochondrial DNA copy number was normalized to nuclear DNA copy number by dividing the number of mitochondrial genomes by the average number of nuclear genomes/cell to derive the mitochondrial copy number/genome/cell. Initially values were calculated for each replicate and a final mean value was calculated and used as a measure of mtDNA copy number.

\subsection{Experiments with patient samples}

RNA from $12 \mathrm{MSI}$ and seven MSS tumours with RNA from matched surrounding tissue was obtained as a kind gift from Professor Andrew Silver's laboratory (Centre for Genomics and Child Heath, Blizzard Institute, QMUL). Characteristics of the patient samples are listed in Table 3.14. The RNA was quantified and cDNA synthesised as described in section 3.6.1. The expression of the antioxidant enzymes in these samples was measured using qRT-PCR as described in section 3.6.2 and using the primers detailed in Table 3.4. The data was analysed as described in 3.6.3. For each sample the experiment was carried out twice and samples were plated in duplicate for each of these experiments. 
Table 3.14

\begin{tabular}{|c|c|c|c|c|c|c|}
\hline Code & MSI/MSS & Gender & $\begin{array}{l}\text { Age at } \\
\text { diagnosis }\end{array}$ & TNM & Dukes' & Site \\
\hline 303 & MSI & M & 47 & T2N2Mx & C & $\begin{array}{l}\text { Proxiaml } \\
\text { colon }\end{array}$ \\
\hline 312 & MSI & $\mathrm{F}$ & 54 & T3N1Mx & C & $\begin{array}{l}\text { Proximal } \\
\text { colon }\end{array}$ \\
\hline 323 & MSI & $\mathrm{F}$ & 82 & T3N0Mx & B & $\begin{array}{c}\text { Proxiamal } \\
\text { colon }\end{array}$ \\
\hline 325 & $\mathrm{MSI}$ & M & 77 & T3N0Mx & $B$ & $\begin{array}{c}\text { Proximal } \\
\text { colon }\end{array}$ \\
\hline 334 & $\mathrm{MSI}$ & M & 76 & T3N1Mx & C & $\begin{array}{l}\text { Proximal } \\
\text { colon }\end{array}$ \\
\hline 339 & MSI & $\mathrm{F}$ & 79 & T4NOMx & $B$ & $\begin{array}{l}\text { Proximal } \\
\text { colon }\end{array}$ \\
\hline 340 & $\mathrm{MSI}$ & M & 73 & T3N1Mx & C & $\begin{array}{l}\text { Proximal } \\
\text { colon }\end{array}$ \\
\hline 354 & MSI & $\mathrm{F}$ & 81 & $\begin{array}{c}\text { Large } \\
\text { villus } \\
\text { adenoma }\end{array}$ & $\begin{array}{c}\text { Large } \\
\text { villus } \\
\text { adenoma }\end{array}$ & Rectum \\
\hline 362 & MSI & $\mathrm{F}$ & 83 & T3N1Mx & C & $\begin{array}{l}\text { Proximal } \\
\text { colon }\end{array}$ \\
\hline 371 & MSI & M & 83 & T3NOMx & B & $\begin{array}{l}\text { Distal } \\
\text { colon }\end{array}$ \\
\hline 375 & $\mathrm{MSI}$ & M & 65 & T3N0Mx & B & $\begin{array}{c}\text { Proximal } \\
\text { colon }\end{array}$ \\
\hline 383 & MSI & M & 20 & T3N0Mx & $B$ & $\begin{array}{l}\text { Proximal } \\
\text { colon }\end{array}$ \\
\hline 66 & MSS & $\begin{array}{c}\text { Not } \\
\text { available }\end{array}$ & $\begin{array}{c}\text { Not } \\
\text { available }\end{array}$ & $\begin{array}{c}\text { Not } \\
\text { available }\end{array}$ & $\begin{array}{c}\text { Not } \\
\text { available }\end{array}$ & $\begin{array}{c}\text { Not } \\
\text { available }\end{array}$ \\
\hline 131 & MSS & $\mathrm{M}$ & 27 & T3N0Mx & $B$ & Rectum \\
\hline 347 & MSS & $M$ & 58 & T4N2Mx & $C$ & $\begin{array}{l}\text { Proximal } \\
\text { colon }\end{array}$ \\
\hline 353 & MSS & $\mathrm{F}$ & 36 & T2N0Mx & A & Rectum \\
\hline 389 & MSS & $M$ & 78 & T3N0Mx & $B$ & Rectum \\
\hline 390 & MSS & $\mathrm{F}$ & 60 & T3N2Mx & $C$ & $\begin{array}{l}\text { Proximal } \\
\text { colon }\end{array}$ \\
\hline 397 & MSS & $\mathrm{F}$ & 77 & T3N0Mx & $B$ & $\begin{array}{l}\text { Distal } \\
\text { colon }\end{array}$ \\
\hline
\end{tabular}

Table 3.14. Patient characteristics of patient samples 


\section{Results}

Chapter 1: Identification and characterization of novel therapeutics for the treatment of MLH1-deficient cancers

Chapter 2: Functional investigation to study the differences between mitochondrial function in MLH1-deficient and proficient cells lines

Chapter 3: Investigation of mitochondrial function in a range of MMR deficient cell lines and in patient tumour samples 


\subsection{Identification and characterization of novel therapeutics for the treatment of MLH1-deficient cancers}

\subsection{Validation of mitochondrial-targeted agents in a range of MLH1-deficient and proficient cell lines.}

\subsubsection{The ROS inducing agent Parthenolide is selectively lethal with MLH1-deficient cell lines}

With the aim of identifying compounds that are selectively lethal with MLH1 deficient cell lines, our lab have previously screened the MLH1-deficient human colorectal cell line HCT116 and its isogenetically matched MLH1-proficient cell line HCT116+chr3, with the Prestwick chemical library (120 small molecules, 90\% marketed drugs and the remaining $10 \%$ bioactive alkaloids). Cell viability was estimated using a luciferasebased ATP assay (CellTiter-Glo) and the effect of each compound on viability was estimated by comparing luciferase readings from drug treated wells with those from wells treated with the compound vehicle, DMSO. "Hit" compounds were identified if treatment caused selective lethality in MLH1-deficient cells, in comparison to MLH1proficient cells. The top hits included Menadione, Podophyllotoxin and Parthenolide. Strikingly, upon analysis, all of these hit compounds have been shown to target mitochondrial function.

Preliminary data previously carried out in our lab outlined above, using synthetic lethal compound screens suggest that the MLH1-deficient cell line, HCT116 is selectively lethal with mitochondrial-targeted agents (Menadione, Parthenolide and Podophyllotoxin), when compared to the isogeneticallymatched MLH1-proficient cell line, HCT116+chr3. Based on this data I initially aimed to validate the drugs identified in these previous screens, in addition to a range of agents known to target various aspects of the mitochondria, in a panel of MLH1-deficient and -proficient cell lines. To this end, I first confirmed the MLH1 status of a panel of cell lines (Table 4.1) (HCT116, A2780cp70, IGROV, MFE-296, SKOV3, SW48, HCT116+chr3, A2780, HT29, MFE-280, SW620) from a variety of tumour types by western blotting (Figure 4.1). 
Table 4.1

\begin{tabular}{|c|c|c|}
\hline Cell line & Tumour type & MLH1 status \\
\hline HCT116 & Colorectal & Deficient \\
\hline A2780cp70 & Ovarian & Deficient \\
\hline AN3CA & Ovarian & Deficient \\
\hline MFE296 & Endometrial & Deficient \\
\hline IGROV & Ovarian & Deficient \\
\hline SKOV3 & Ovarian & Deficient \\
\hline SW48 & Colorectal & Proficient \\
\hline HCT116+chr3 & Colorectal & Proficient \\
\hline A2780 & Ovarian & Proficient \\
\hline HT29 & Colorectal & Proficient \\
\hline MFE280 & Endometrial & Proficient \\
\hline SW620 & Colorectal & Pro \\
\hline
\end{tabular}

Table 4.1. Panel of cell lines used in this study with the MLH1 status and tumour type from which they were derived. 
Figure 4.1
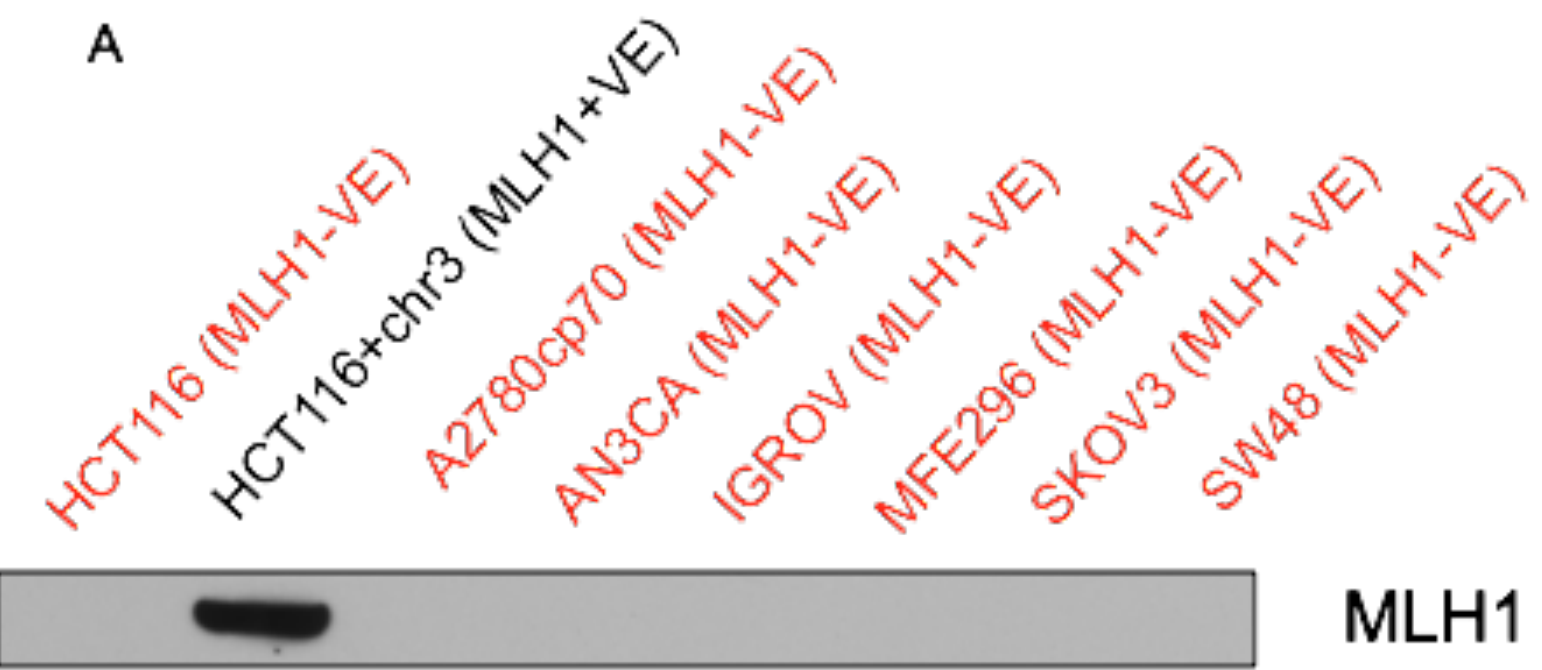

MLH1

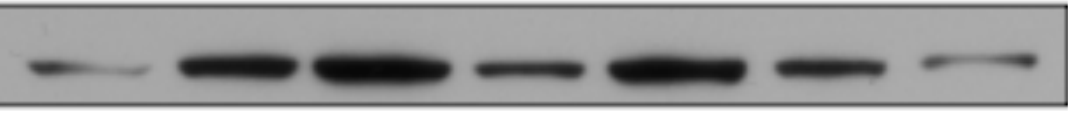

$\beta$-actin

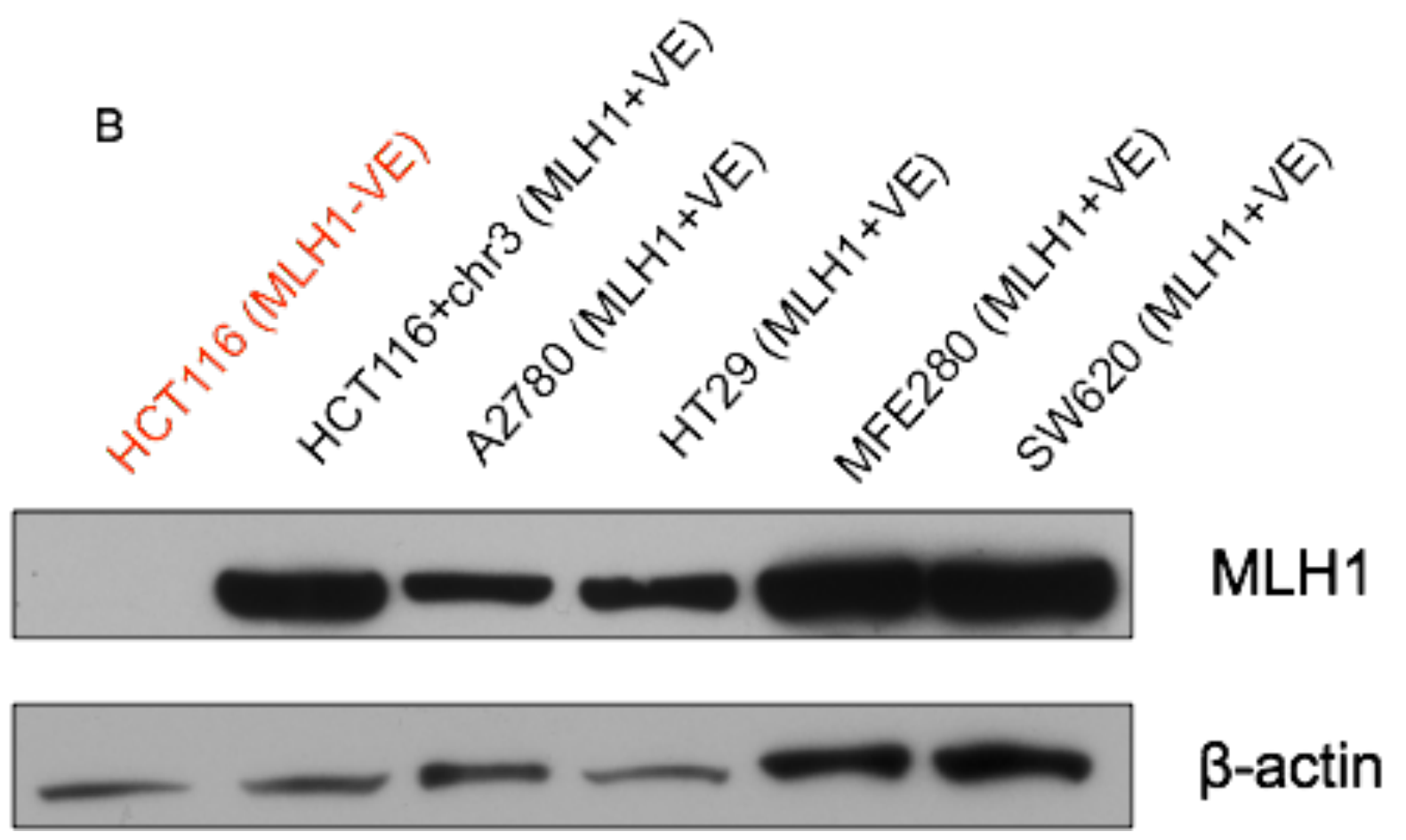


Figure 4.1. Validation of MLH1 expression status in a panel of MLH1-deficient and proficient cell lines.

Protein lysates were isolated from a panel of MLH1-deficient (HCT116, A2780cp70, AN3CA, MFE296, SKOV3 and SW48) and proficient (HCT116+chr3, A2780, HT29, MFE280 and SW620) cell lines and analyzed by western blotting using MLH1 and $\beta$-actin primary antibodies. (A) Western blotting to confirm the MLH1 status of a panel of MLH1-deficient cell lines. The MLH1proficient cell line $\mathrm{HCT} 116+\mathrm{chr} 3$ was used as a control. (B) Western blotting to confirm the MLH1 status of a panel of MLH1-proficient cell lines. The MLH1-deficient cell line HCT116 was used as a control. 
I next treated our panel of MLH1-deficient and MLH1-proficient cell lines using shortterm cell viability assays with increasing concentrations of the 'hit' compounds (Parthenolide, Menadione and Podophllotoxin) identified in the drug screen which all target mitochondrial function. Five days post drug treatment, cell viability was estimated using a luciferase-based ATP assay (CellTiter-Glo; Promega). The effect of each compound was determined by comparing luciferase readings from drug treated wells with those from wells treated with the compound vehicle (DMSO). These experiments validated that the ROS inducing drug Parthenolide exhibited the most consistent selective toxicity between MLH1-deficient and -proficient cell lines in both the isogenetically matched cell lines HCT116 (MLH1-VE) and HCT116+chr3 (MLH1+VE) (Figure 4.2) as well as in a panel of genetically diverse MLH1-deficient and -proficient cell lines from a variety of tumour types (Figure 4.3). 
Figure 4.2

A

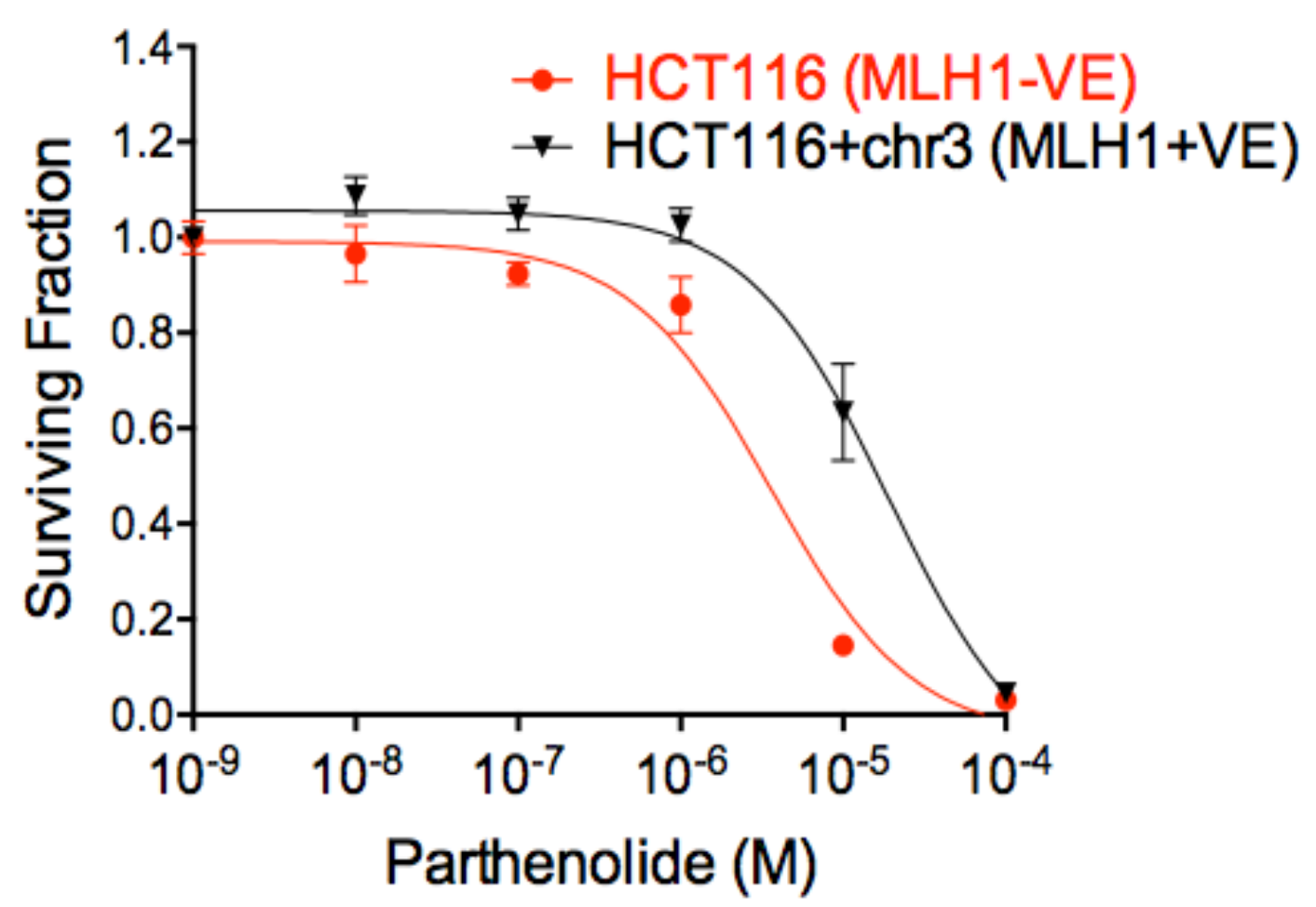

B

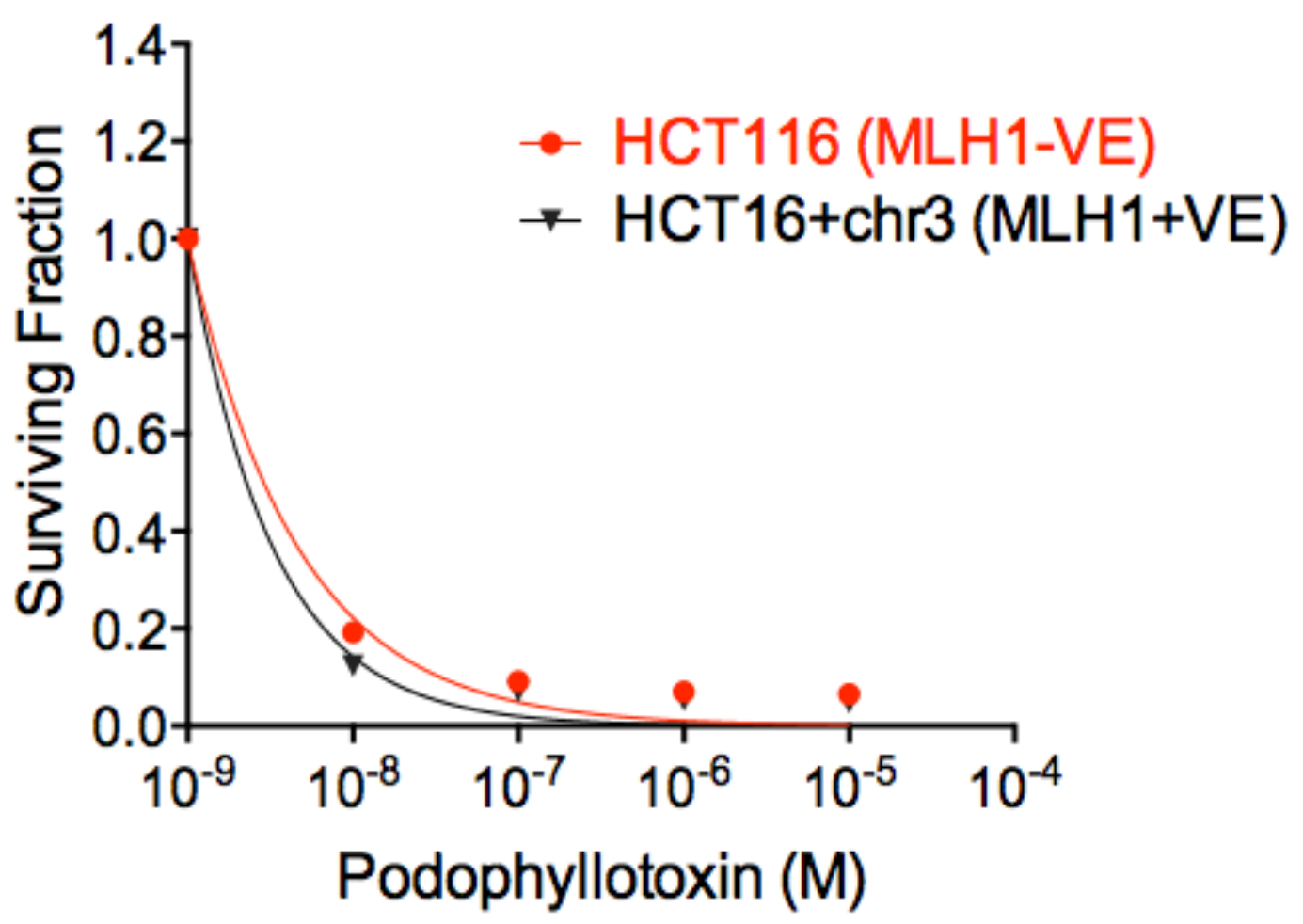


$\mathrm{C}$

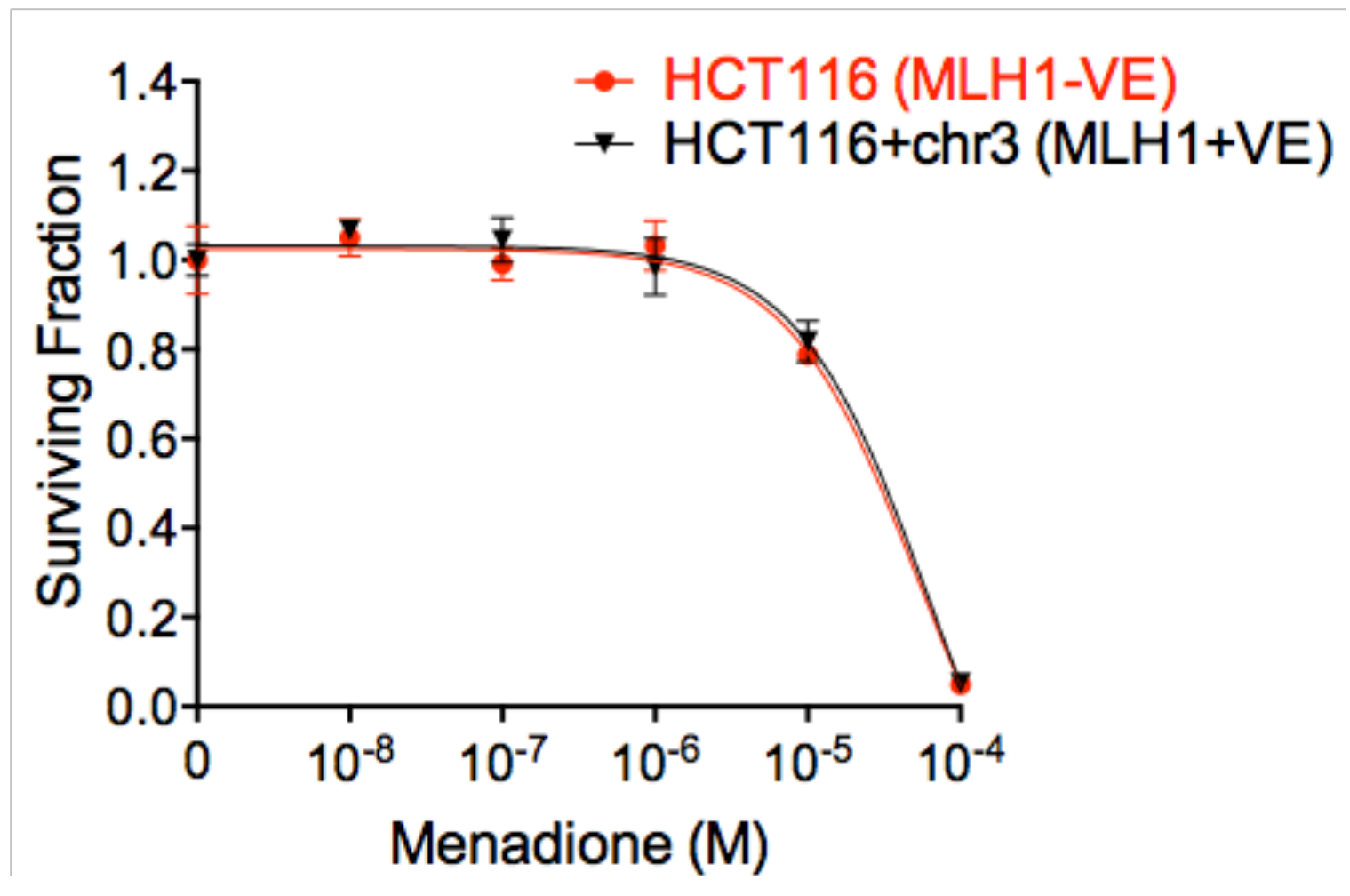

Figure 4.2. The ROS inducing agent Parthenolide is synthetically lethal with the MLH1deficient cell line HCT116 compared to its isogenetically matched MLH1-proficient cell line.

(A-C) Short-term survival curves are shown upon continuous exposure to increasing concentrations of Parthenolide. After 5 days cell viability was estimated using the ATP assay, CellTiter-Glo. The isogenetically matched cell lines HCT116 which is MLH1-deficient and HCT116+chr3 which is MLHL1- proficient were treated with increasing concentrations of $(A)$ Parthenolide (B) Podophyllotoxin (C) Menadione. Graphs shown here are one representative example of three independent experiments carried out in triplicate. Error bars represent standard deviation of the mean. 
Figure 4.3

A

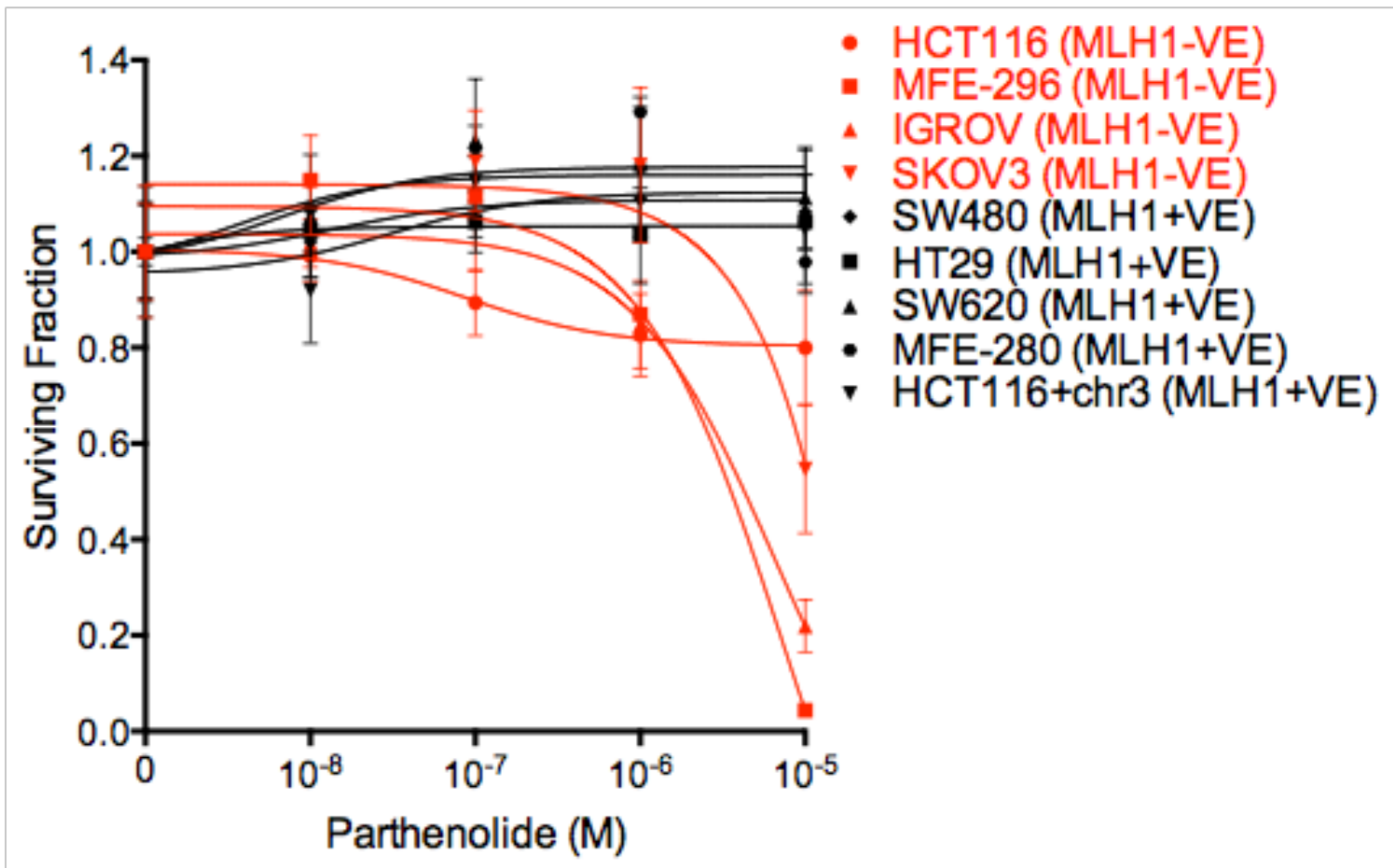

B

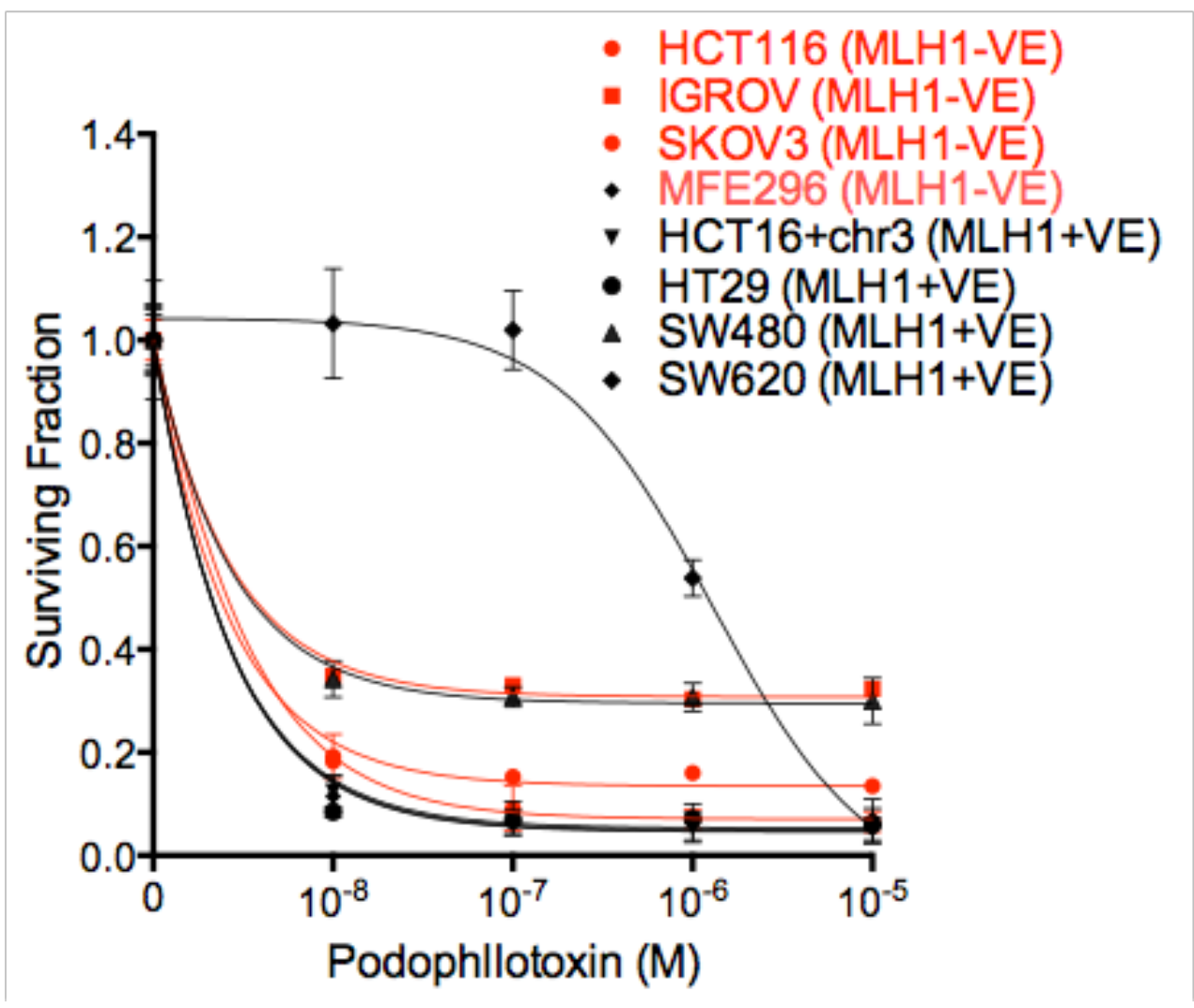


$\mathrm{C}$

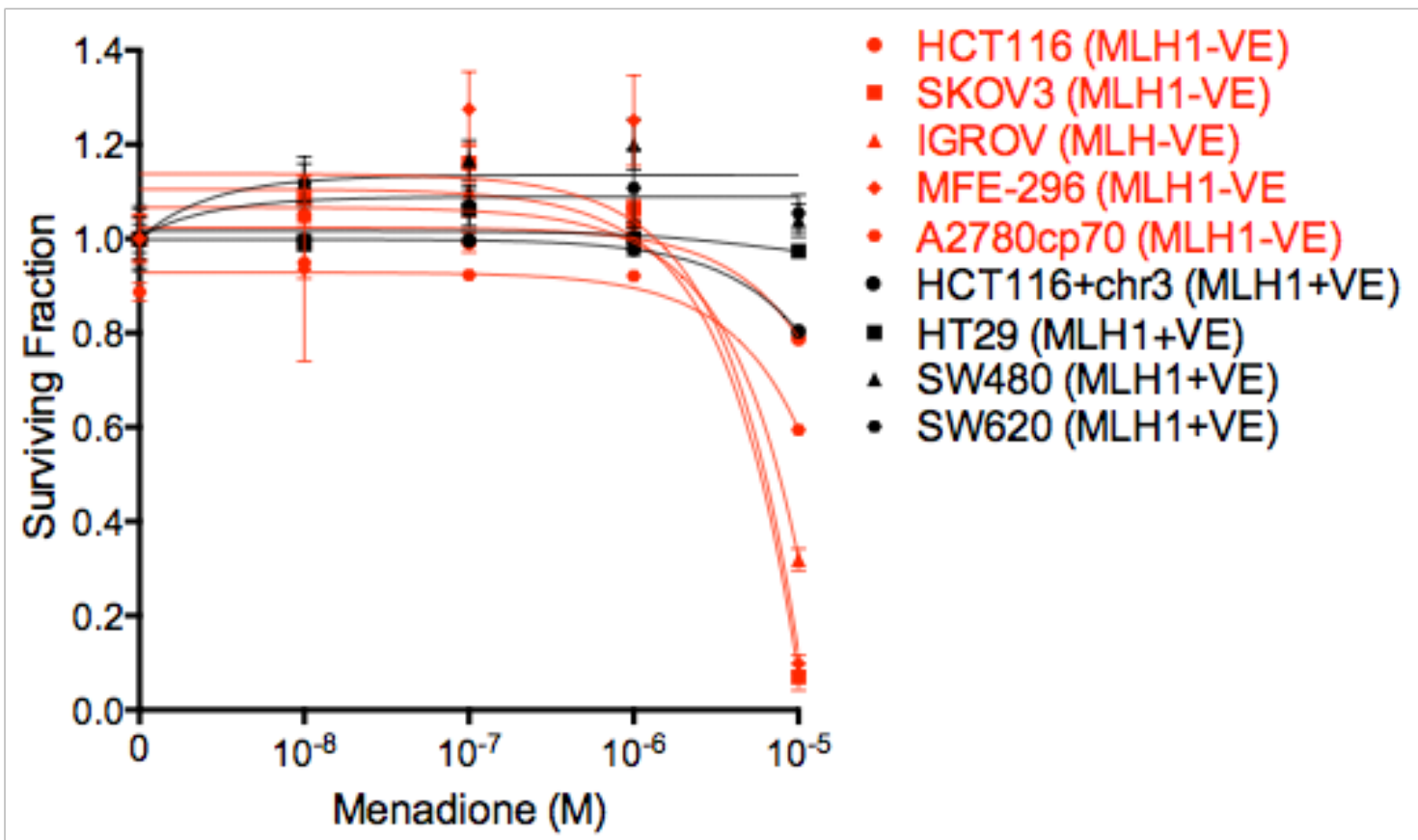

Figure 4.3. The ROS inducing agent Parthenolide is synthetically lethal with MLH1-deficient cell lines in a panel of cell lines from a range of tumour types.

(A-C) The MLH1-deficient cell lines HCT116, SKOV3, IGROV, MFE-296, A2780cp70 and the MLH1-proficient cell lines HCT116+chr3, SW480, SW620 and HT29 under continuous exposure to increasing concentrations of (A) Parthenolide (B) Podophyllotoxin (C) Menadione. Graphs shown here are one representative example of three independent experiments carried out in triplicate. Error bars represent standard deviation of the mean. 
4.1.1.2 MLH1-deficient cell lines are only synthetically lethal with Parthenolide and not other drugs targeting mitochondrial function

To determine, whether mitochondrial-targeted drugs in general are selective for loss of MLH1, the same panel of MLH1-proficient and -deficient cell lines was treated with a range of mitochondrial-targeted compounds including Betulinic acid, Dichloroacetate, 2Deoxy-D-glucose, ABT 737, and Lonidamine (Figure 4.4A-4.4E), (Table 4.2). These drugs act on a variety of mitochondrial functions (Table 4.2) but did not show selectivity with MLH1 deficiency. 
Table 4.2

\begin{tabular}{|c|c|}
\hline Drug & Mechanism of Action \\
\hline Parthenolide & $\begin{array}{c}\text { ROS inducing agent } \\
\text { Activates NADPH oxidase } \\
\text { Inhibits SOD1 and } \\
\text { catalase }\end{array}$ \\
\hline Menadione & $\begin{array}{l}\text { ROS inducing agent } \\
\text { Futile redox cycling }\end{array}$ \\
\hline B-lapachone & $\begin{array}{c}\text { NQO1 dependent ROS } \\
\text { induction }\end{array}$ \\
\hline 2-Deoxy-D-Glucose & $\begin{array}{l}\text { Inhibitor of hexokinase } \\
(\mathrm{HK})\end{array}$ \\
\hline Dichloroacetate & $\begin{array}{c}\text { Inhibitor of pyruvate } \\
\text { dehydrogenase kinase } \\
\text { (PDK) }\end{array}$ \\
\hline Podophyllotoxin & $\begin{array}{l}\text { Inhibitor of Topoisomerase } \\
\text { II }\end{array}$ \\
\hline ABT-737 & $\begin{array}{l}\text { Modulator of BCL-2 } \\
\text { protein family }\end{array}$ \\
\hline Lonidamine & $\begin{array}{c}\text { Adenine nucleotide } \\
\text { translocase ligand (ANT) }\end{array}$ \\
\hline Betulinic acid & $\begin{array}{c}\text { Interacts with permeability } \\
\text { transition pore complex } \\
\text { (PTPC) }\end{array}$ \\
\hline
\end{tabular}

Table 4.2. Mechanism of action of mitochondrial-targeted agents [373]. 
Figure 4.4

A

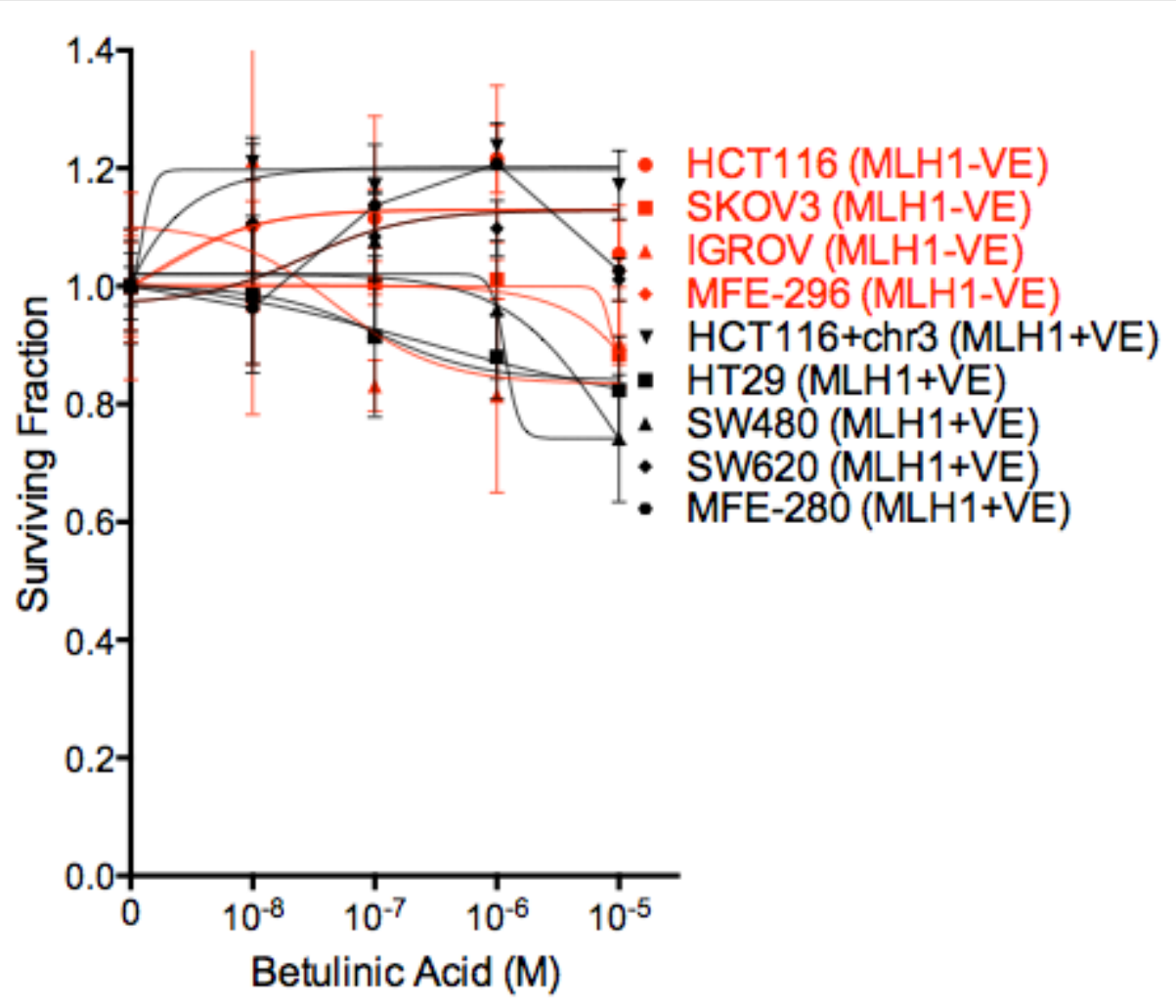

B

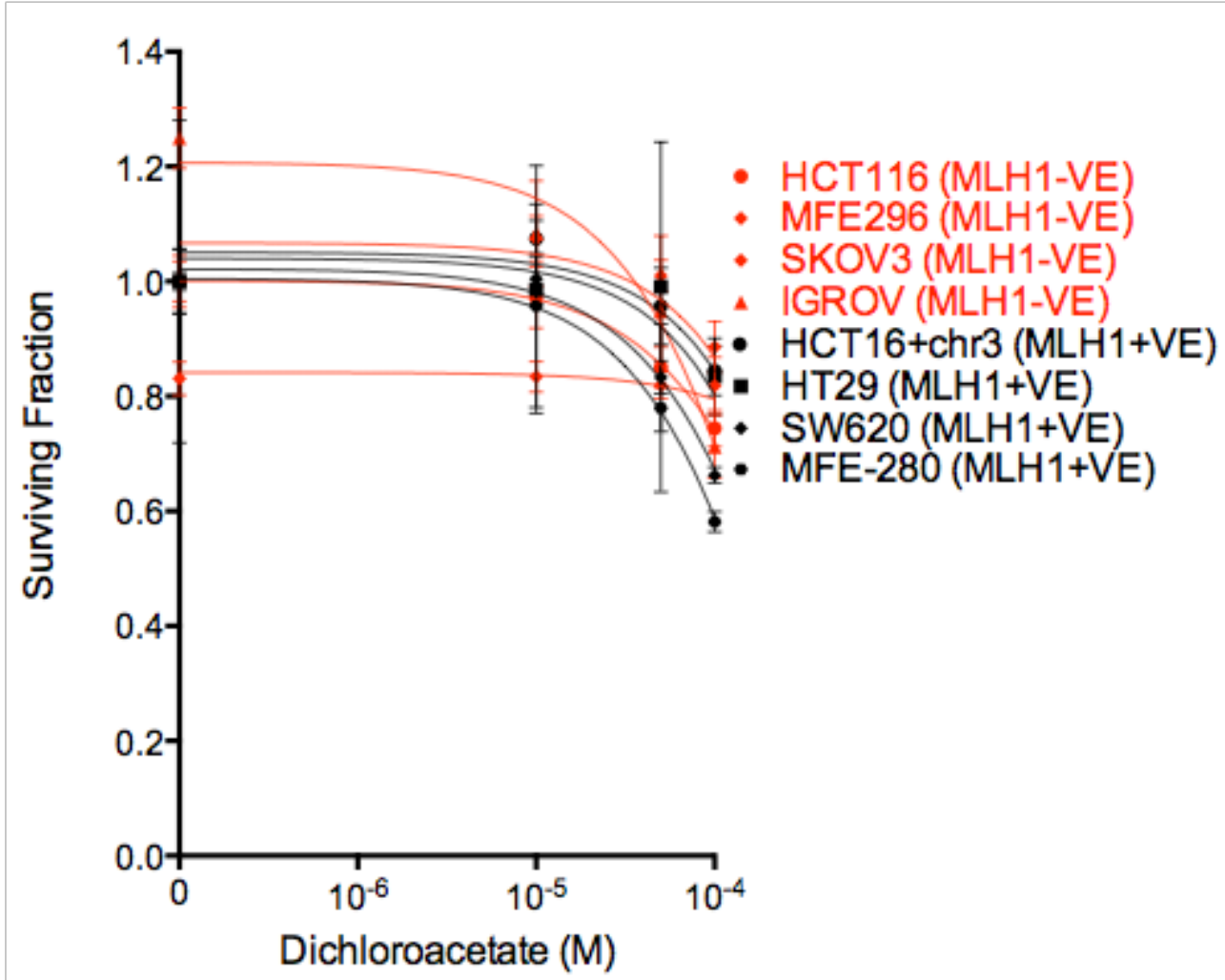


C

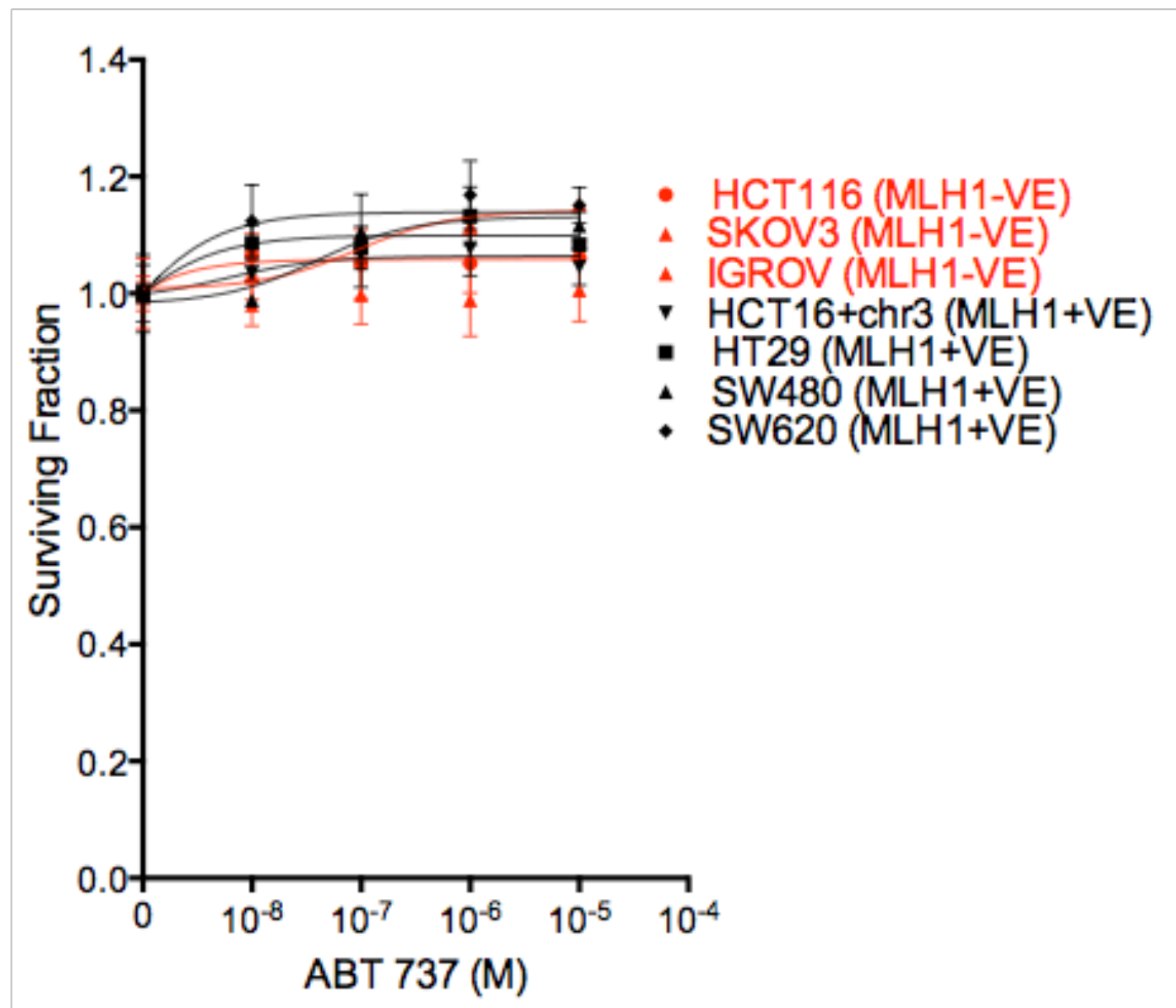

D

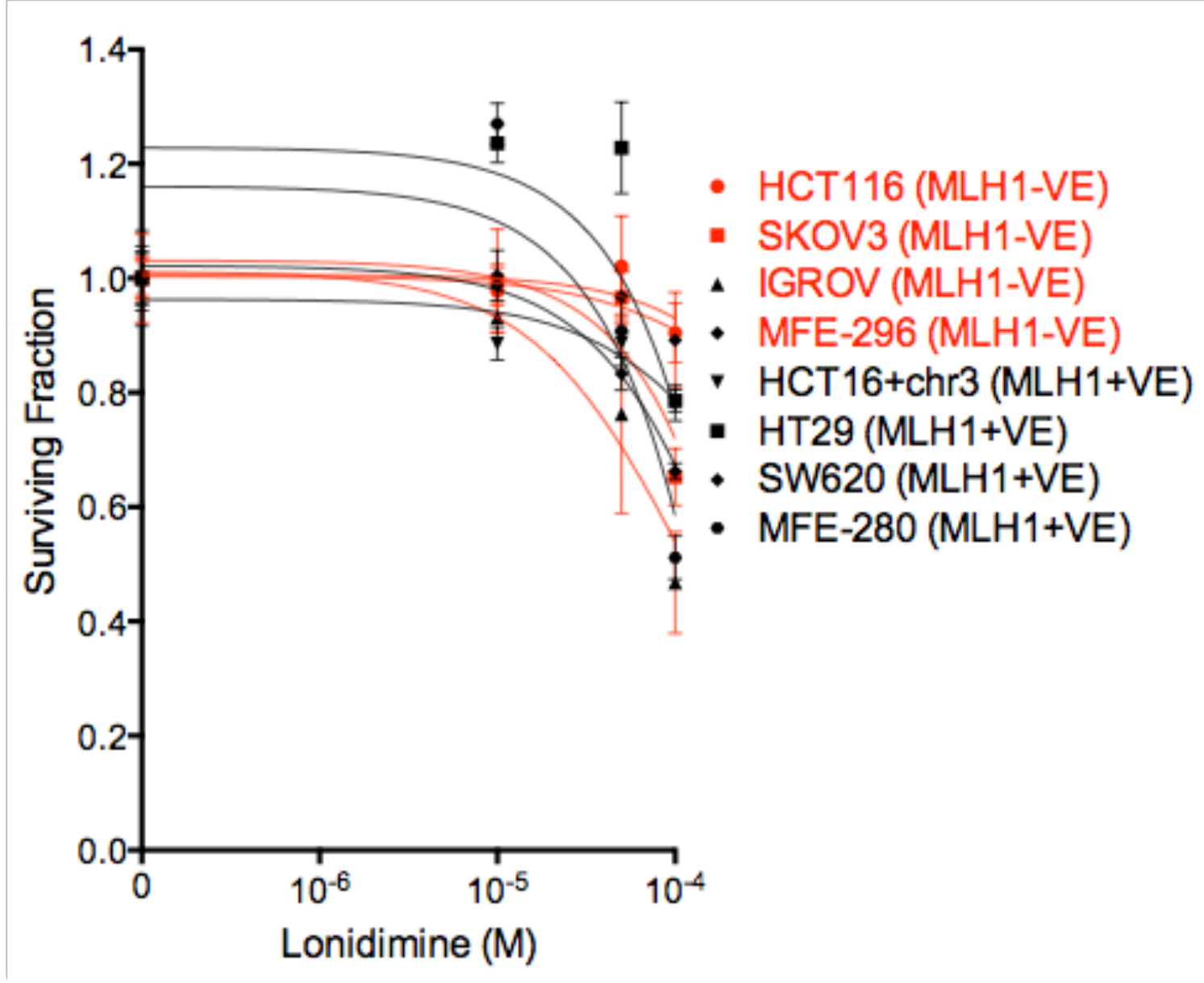




\section{E}

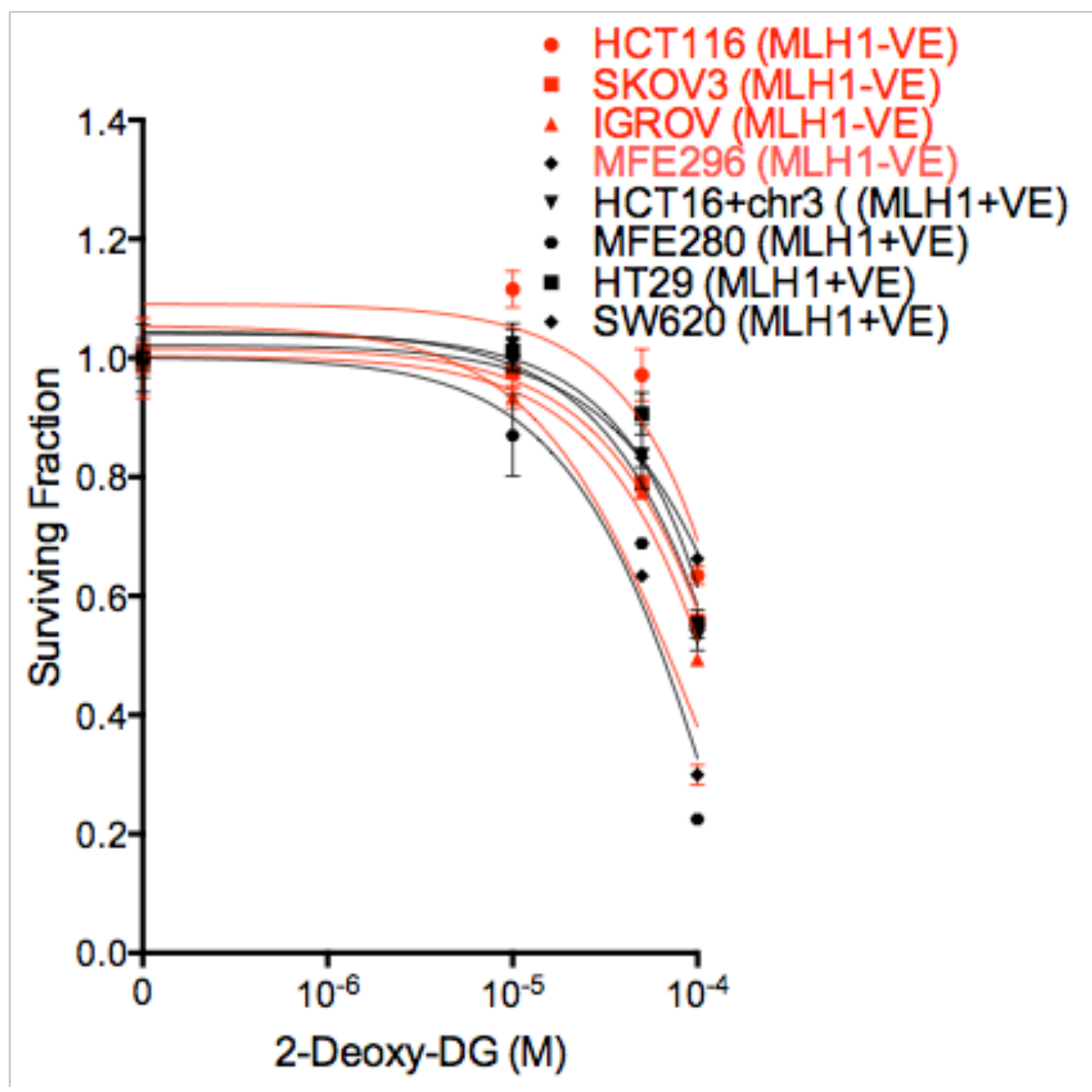

Figure 4.4. MLH1 loss does not confer selectivity to a range of mitochondrial-targeted agents.

(A-E) Investigation into the selectivity of the mitochondrial-targeted drugs, ABT 737, 2-Deoxy-Dglucose, Lonidamine, Betulinic acid and Dichloroacetate in MLH1-deficient cell lines. Shortterm survival curves are shown under continuous exposure to increasing concentrations of the mitochondrial-targeted agents. After 5 days of exposure to the drugs, cell viability was estimated using the ATP assay CelltireGLo. Short term cell viability curves of the MLH1-deficient cell lines HCT116, SKOV3, IGROV, MFE-296, A2780cp70 and the MLH1-proficient cell lines HCT116+chr3, SW480, SW620 and HT29 under continuous exposure to (A) Betulinic acid, (B) Dichloroacetate, (C) ABT 737, (D) Lonidamine, (E) 2-Deoxy-D-glucose (5 days). Error bars represent standard deviation of the mean. Graphs shown here are representative of three independent experiments carried out in triplicate. 


\subsubsection{Investigating the mechanism behind the synthetic lethal relationship between Parthenolide treatment and MLH1 loss.}

\subsubsection{Increased ROS production in MLH1-deficient cell lines treated with Parthenolide}

Parthenolide has been established to be a ROS inducing agent and I observed a synthetic lethal relationship with Parthenolide and MLH1-deficient cell lines. Therefore I next investigated whether this selectivity was due to ROS induction. To this end, I used the dye Dihydroethidium (DHE) to measure ROS production in MLH1-deficient (HCT116) and -proficient (HCT116+chr3) cell lines treated with vehicle control (DMSO) and Parthenolide $(10 \mu \mathrm{M})$. The cell lines were treated with either DMSO, Parthenolide or three different concentrations of the Complex III inhibitor Antimycin which is known to induce ROS (positive control). Cellular ROS levels were measured using the dye DHE which becomes oxidized to ethidium in the presence of ROS and emits red fluorescence. This increased fluorescence was measured on a high-throughput fluorescent microscope (MitoXpress). We observed a gradual increase in fluorescence with increasing concentrations of Antimycin (Figure 4.5A) confirming that this method for measuring ROS was robust. Our results indicated that there was no significant difference in baseline ROS levels between MLH1-deficient and -proficient cell lines treated with DMSO (Figure 4.5B) but upon addition of Parthenolide, we observed a significant increase in ROS production in the MLH1-deficient cell line HCT116 compared to the MLH1-proficient cell line HCT116+chr3 (Figure 4.5C). To further investigate this, I also carried out short-term cell viability assays using the same cell lines treated with Parthenolide, with and without the ROS scavenger, N-acetylcysteine (NAC; Figure 4.5D). I observed that addition of NAC $(1 \mathrm{mg} / \mathrm{ml})$ rescued the selective lethality of Parthenolide in the HCT116 cells and rescued cell viability to approximately the level of the MLH1-proficient cell line, HCT116+chr3 This observation confirmed that Parthenolide induces selective lethality in MLH1-deficient cell lines through accumulation of ROS.

Taken together, these experiments displaying increased ROS production in the MLH1deficient cell line upon Parthenolide treatment and the fact that the ROS scavenger 
NAC is able to rescue this selectivity strongly suggests that the mechanism of selectivity of Parthenolide with MLH1-deficient cell lines is at least in part likely to be related to ROS. These experiments highlighted that ROS metabolism could in some way be different in the MLH1-deficient cell lines compared to the MLH1-proficient cell lines. 
Figure 4.5

A

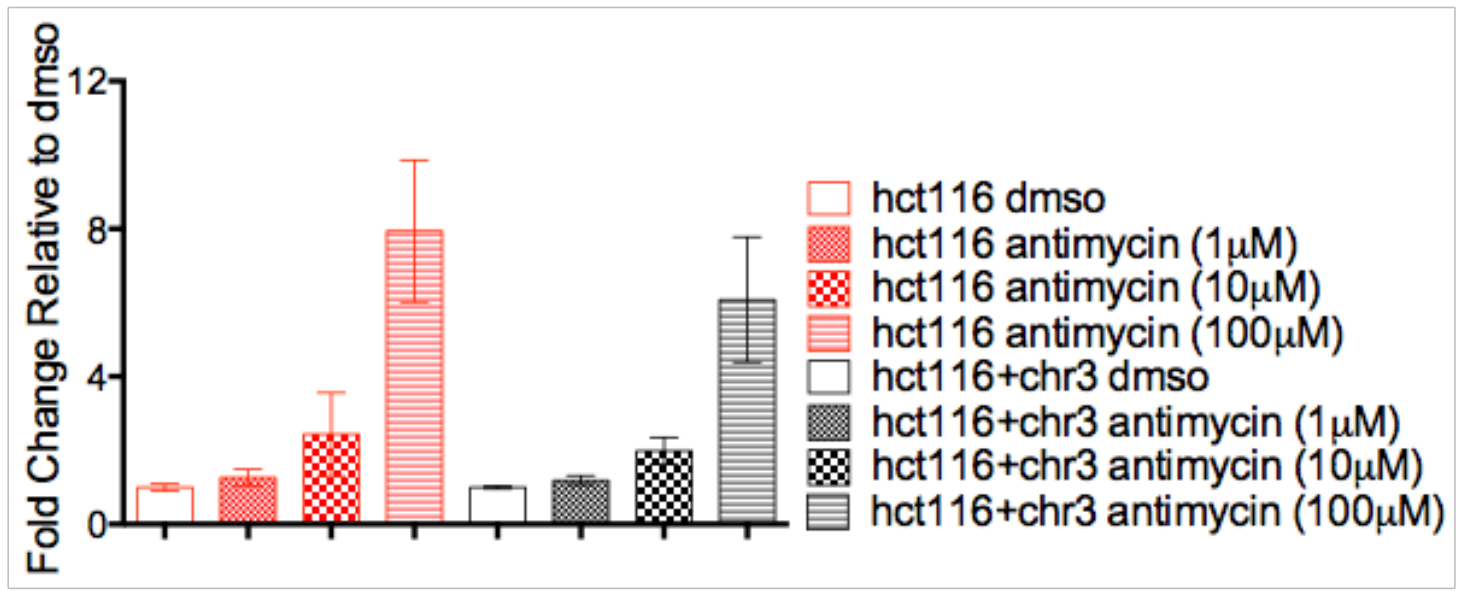

B

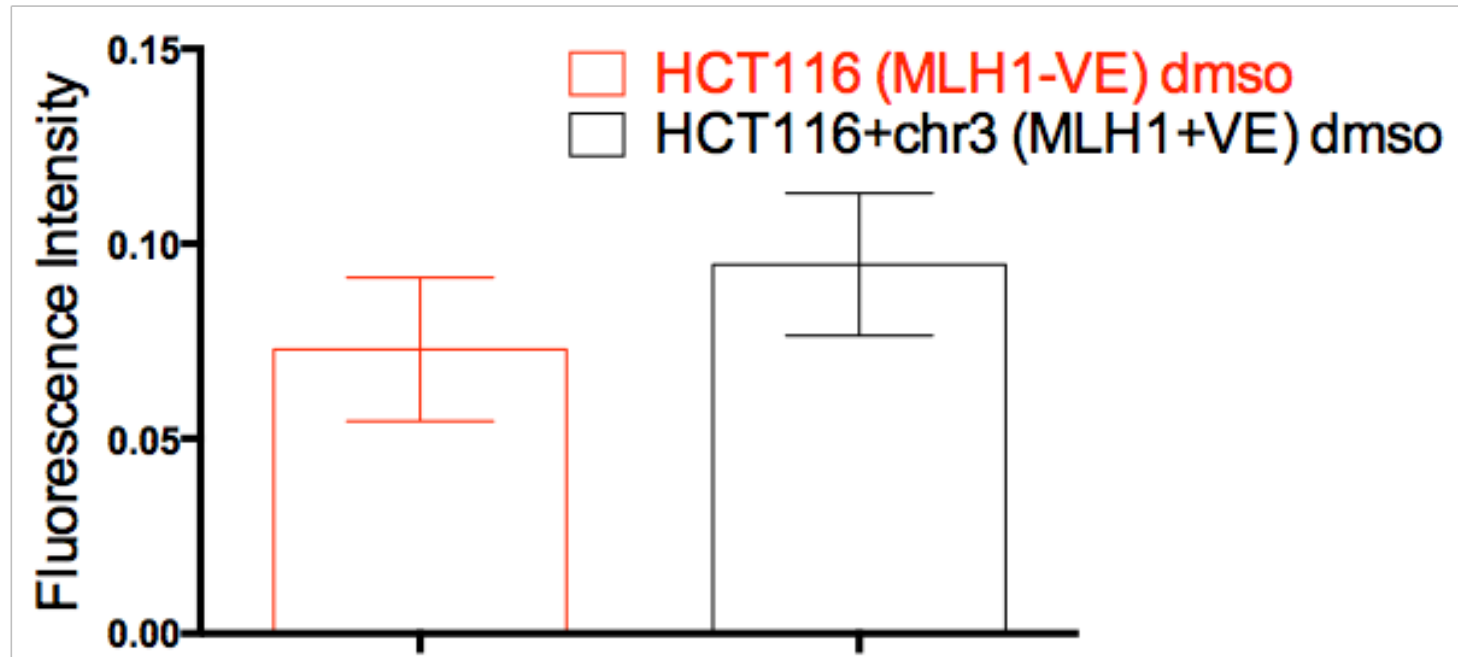


C

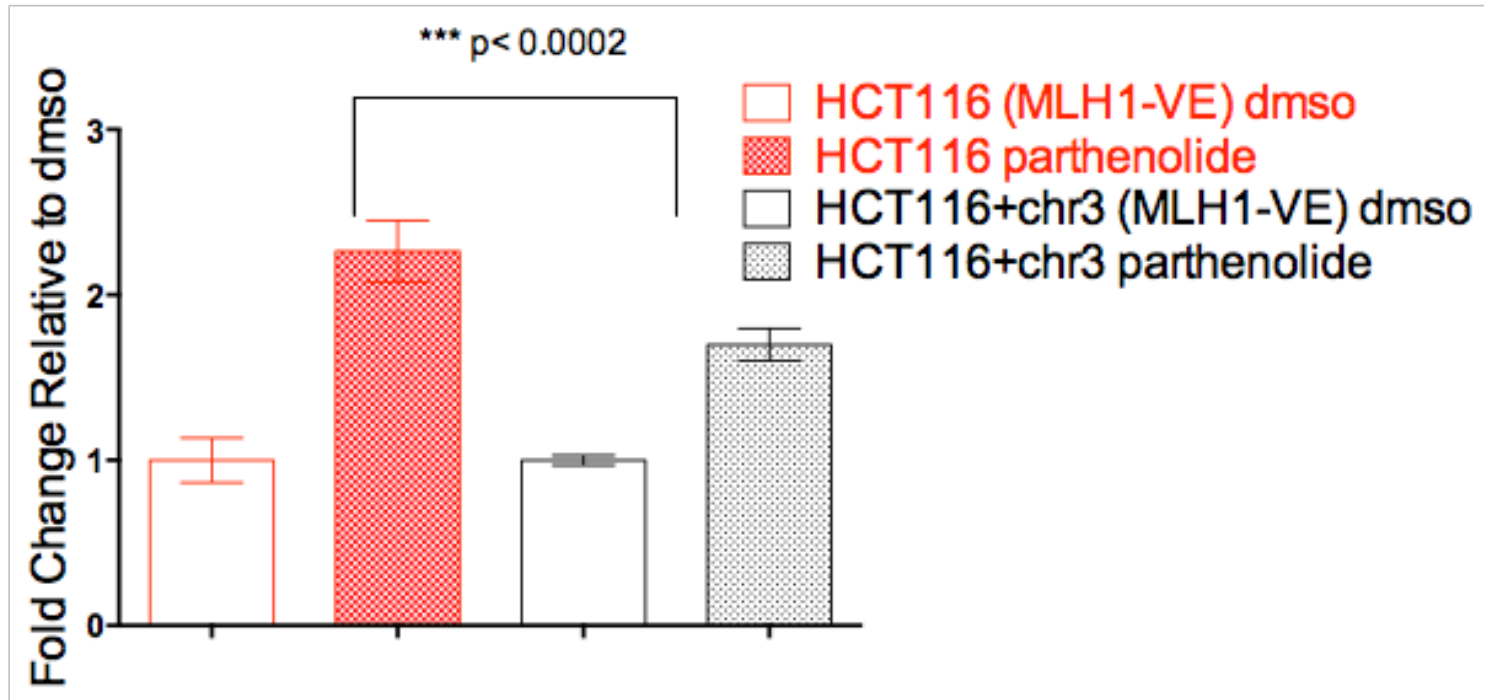

D

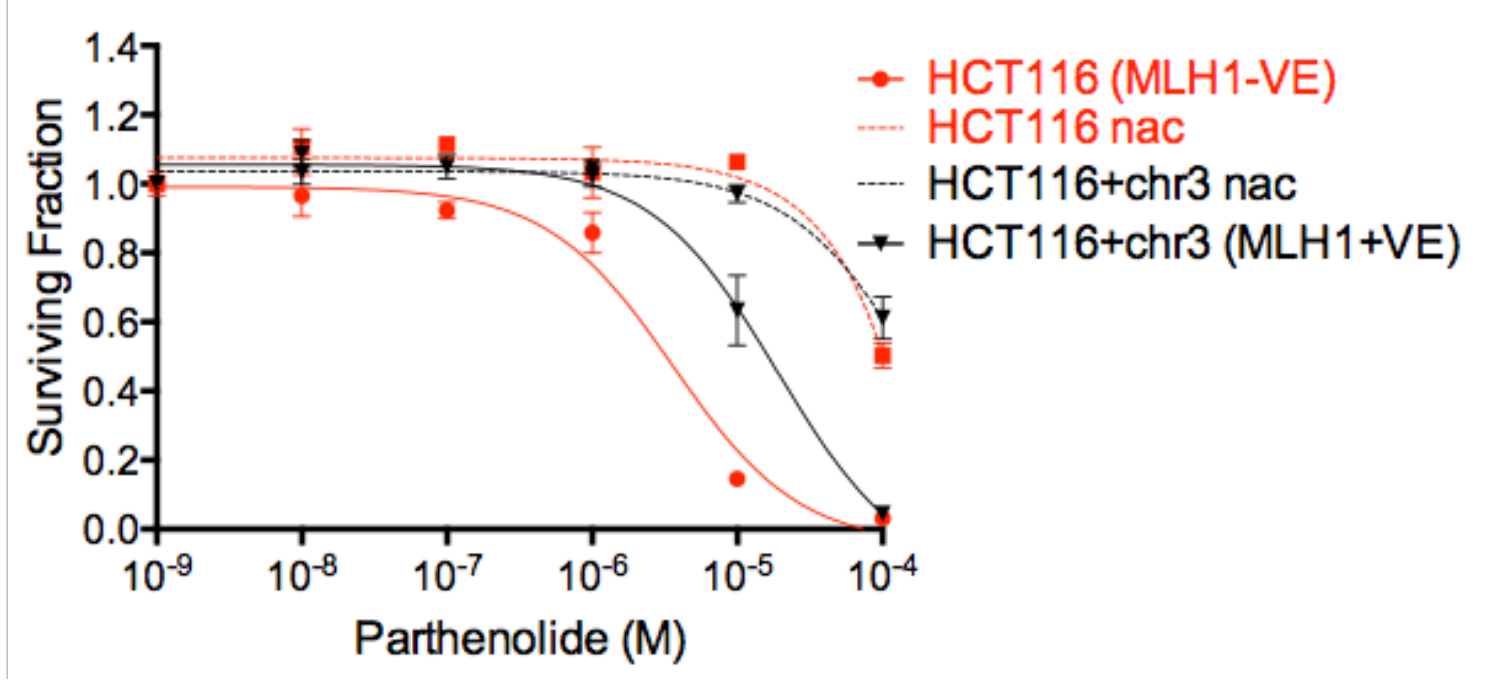


Figure 4.5. The mechanism of action and selectivity of Parthenolide with MLH1-deficient cell lines is related to ROS accumulation.

The MLH1-deficient HCT116 cell line and its isogenetically matched MLH1-proficient cell line HCT116+chr3 were grown for 24 hours and then treated with the vehicle control DMSO, Parthenolide $(10 \mu \mathrm{M})$ or the positive control Antimycin, $(1 \mu \mathrm{g}, 10 \mu \mathrm{g}$ and $100 \mu \mathrm{g})$ for $30 \mathrm{~min}$. The cells were washed and treated with $10 \mu \mathrm{M}$ DHE, fluorescence was measured on the MitoXpress fluorescent microscope as a measure of ROS production. (A) There was no significant difference between the baseline (DMSO treated) ROS levels in MLH1-deficient and proficient isogeneticallymatched cell lines. (B) There is a significant increase in ROS levels in the MLH1deficient cell line HCT116 compared to the MLH1-proficient cell line HCT116+chr3 upon addition of Parthenolide. (C) ROS levels increase in proportion to increasing concentrations of the positive control Antimycin confirming the validity of this assay for measuring ROS levels. (D) The selectivity of MLH1-deficient cell lines with Parthenolide is rescued by NAC. Short term cell survival curves are shown here under continuous exposure to Parthenolide with (dotted lines) or without NAC (solid lines). (A-C) Graphs shown here are results from two independent experiments carried out in triplicate. Error bars represent standard error of the mean. (D) The graph shown here is one representative example of three experiments carried out in triplicate. Error bars represent standard deviation. 


\subsubsection{2}

MLH1-deficient cell lines do not have a synthetic lethal relationship with other ROS inducing agents.

Thus far my results suggest that the mechanism of selectivity of Parthenolide with MLH1 loss is related to ROS and possibly due to ROS accumulation, so I decided to investigate whether MLH1 loss is selectively lethal with other ROS inducing drugs. I treated the isogenetically matched cell line HCT116 (MLH1-deficient) and HCT116+chr3 (MLH1-proficient) with increasing concentrations of the ROS inducing agents Menadione and $\beta$-lapachone with and without the addition of the ROS scavenger NAC and compared the surviving fraction from short term cell viability assays (Figure 4.6A and 4.6B). Overall there was no selective toxicity of these ROS inducing agents with MLH1 loss. Of note, there were several experiments where MLH1 loss was selectively lethal with Menadione and $\beta$-lapachone but my overall conclusion is based on the fact that $\mathrm{I}$ did not observe this selectivity in a reproducible manner across several experiments. The non-selective cell death seen in these experiments was rescued by NAC confirming that the ROS agents used in these experiments did indeed induce ROS and kill the cells by producing ROS. Similar cell viability assays were also carried out in a panel of MLH1-proficient and deficient cell lines (Figure 4.6C and 4.6D) and again overall there was no selective toxicity across all the MLH1-deficient cell lines when treated with Menadione and $\beta$-lapachone. It is worth noting that the majority of MLH1deficient cell lines amongst a panel of cell lines did result in selective toxicity with Menadione and $\beta$-lapachone. These results could be attributed to the different ways in which these agents induce ROS. 
Figure 4.6

A

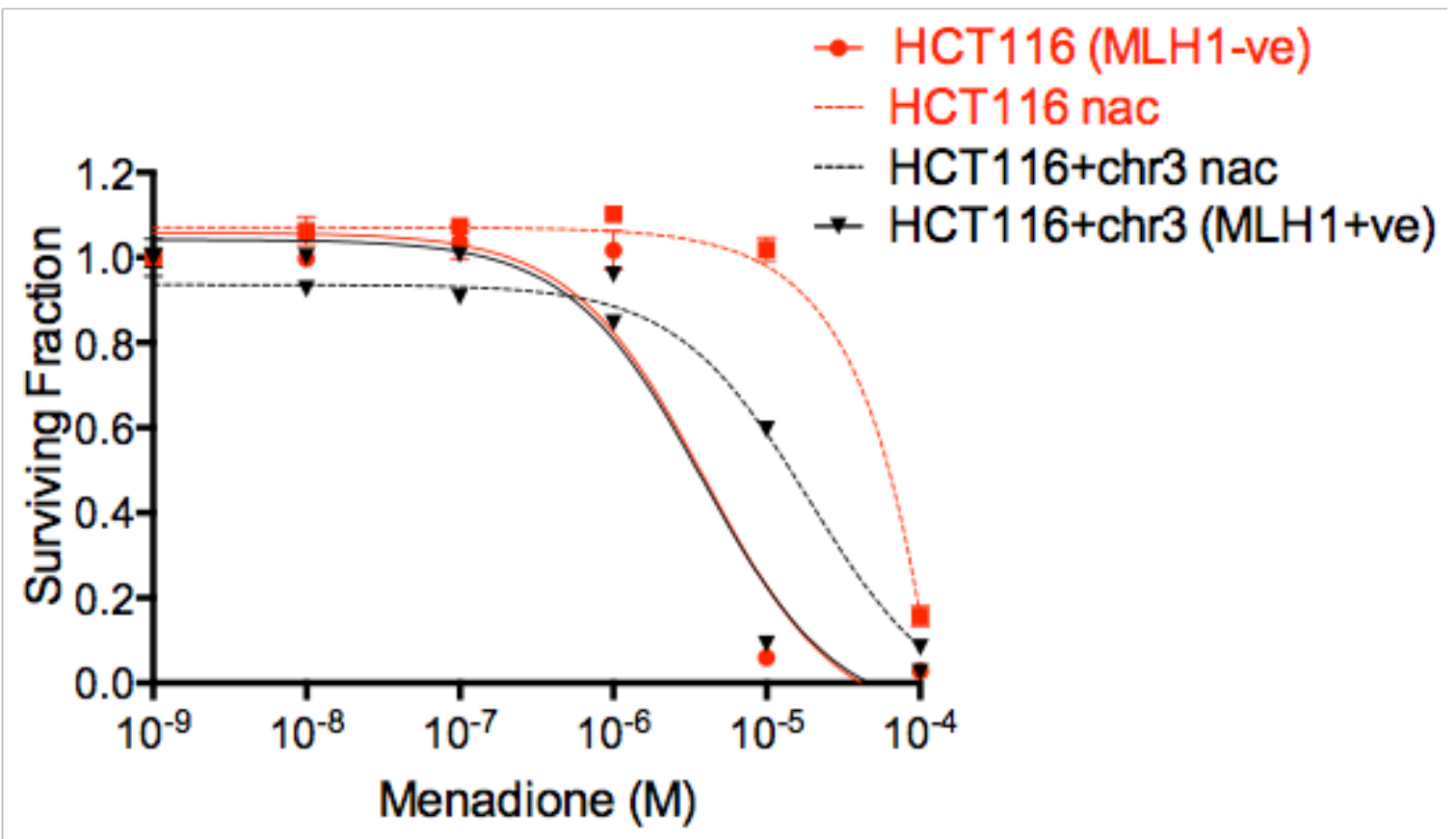

B

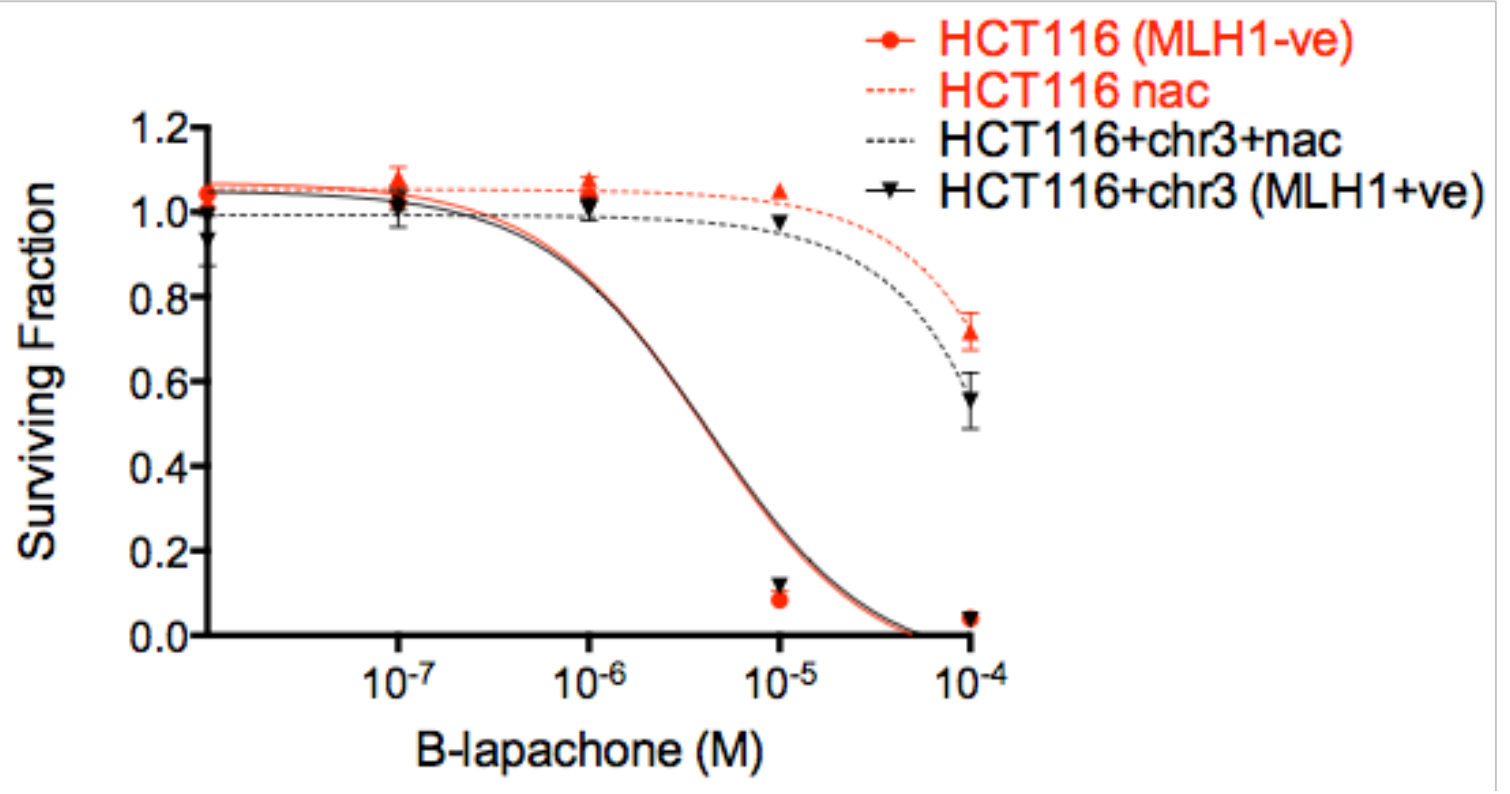


C

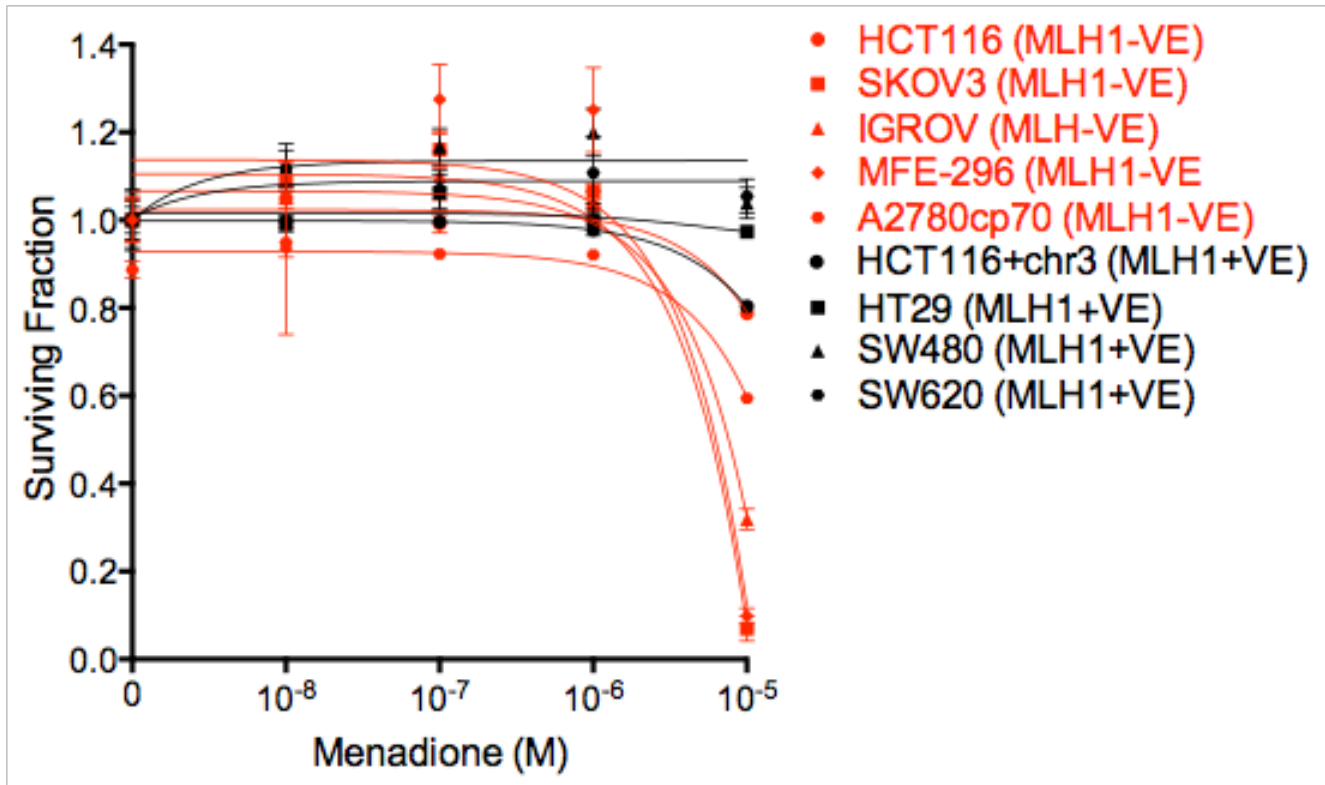

D

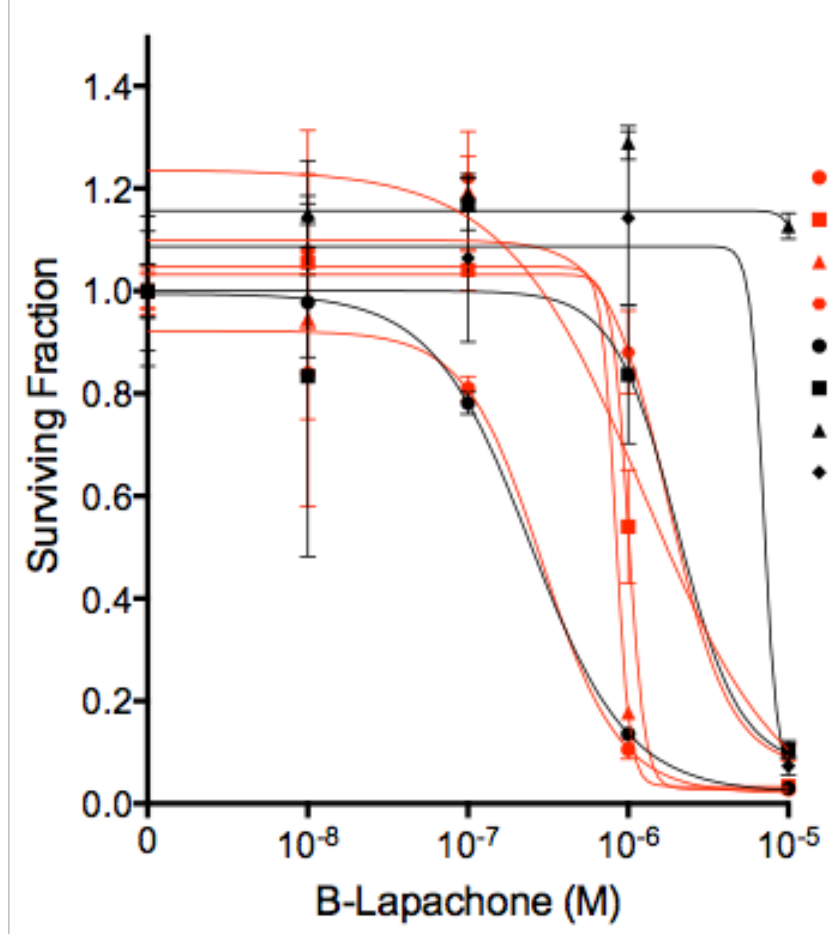

HCT116 (MLH1-ve)

SKOV3 (MLH1-ve)

IGROV (MLH-ve)

- MFE-296 (MLH1-ve)

- HCT116+chr3 (MLH1+ve)

- HT29 (MLH1+ve)

- SW480 (MLH1+ve)

- SW620 (MLH1+ve) 
Figure 4.6. MLH1 loss is not synthetically lethal with other ROS inducing agents.

(A-D) Investigation of the ROS inducing drugs, Menadione and $\beta$-lapachone in MLH1-deficient and-proficient cells. Short term survival curves are shown under continuous exposure to increasing concentrations of the ROS inducing agents After 5 days cell viability was estimated using the ATP assay CelltireGLo. (A, B) Short-term cell viability curves of the isogeneticallymatched cell lines HCT116 (MLH1-deficient) and HCT116+chr3 (MLH1-VE) treated with (A) Menadione +/- NAC and (B) $\beta$-lapachone +/- NAC. (C,D) Short-term cell viability curves of the MLH1-deficient cell lines HCT116, SKOV3, IGROV, MFE-296, A2780cp70 and the MLH1proficient cell lines HCT116+chr3, SW480, SW620 and HT29 under continuous exposure to (C) Menadione and (D) $\beta$-lapachone. Error bars represent standard deviation of the mean. Graphs shown here are representative of three independent experiments carried out in triplicate. 


\subsection{Functional investigation to study the differences between mitochondrial function in MLH1-deficient and proficient cells lines}

\subsubsection{Investigating the antioxidant defense system in MLH1 deficiency}

4.2.1.1 Decreased gene and protein expression of the transcription factor NRF2 and the downstream antioxidant defense enzymes

Thus far I observed that MLH1-deficient cells were sensitive to Parthenolide due to ROS induction but the same sensitivity was not observed with other ROS inducing agents,. Parthenolide is known to act on members of the antioxidant defense system therefore I next investigated whether the cellular antioxidant response could be different upon MLH1 loss..

To this end I measured the gene expression of the transcription factor NRF2 (NFE2L2), which is responsible for coordinating the antioxidant defense system of cells, as well as several downstream antioxidant enzymes (SOD1, catalase, and GPX1 by qRT-PCR (Figure 4.7A-4.7D). I observed a significant decrease in the expression of NRF2, SOD1, Catalase and GPX1 (Figure 4.7A-4.7D) in the MLH1-deficient cell line HCT116 compared to its isogenetically matched pair HCT116+chr3 Next, I validated these observations by western blotting observing decreased protein levels of NRF2, catalase and GPx1 in the MLH1-deficient cells (Figure 4.8A-4.8D). 


\section{Figure 4.7}

A

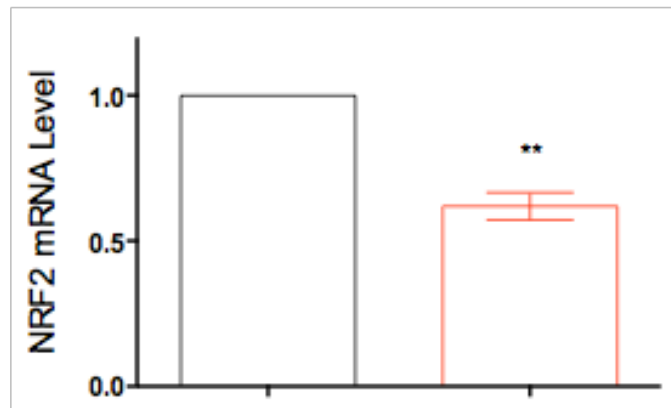

C

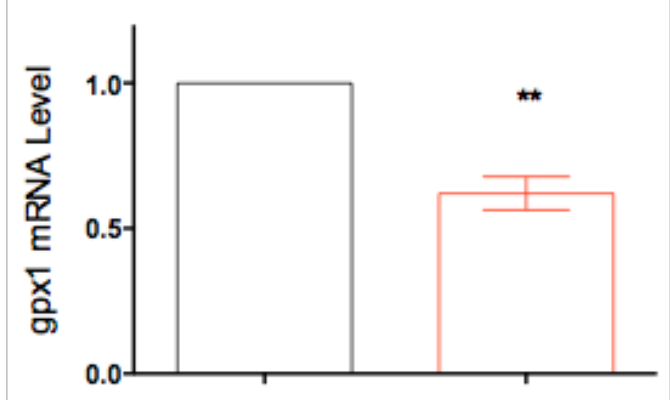

B

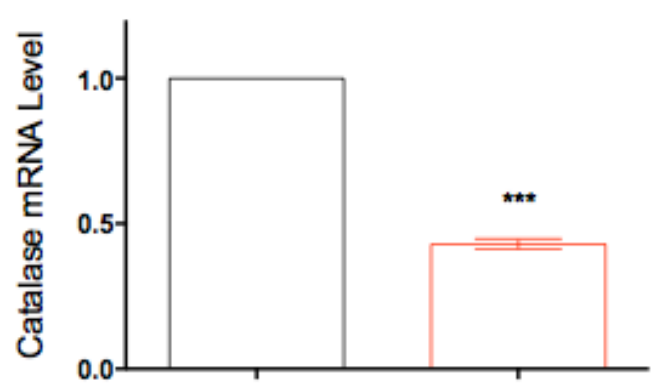

D

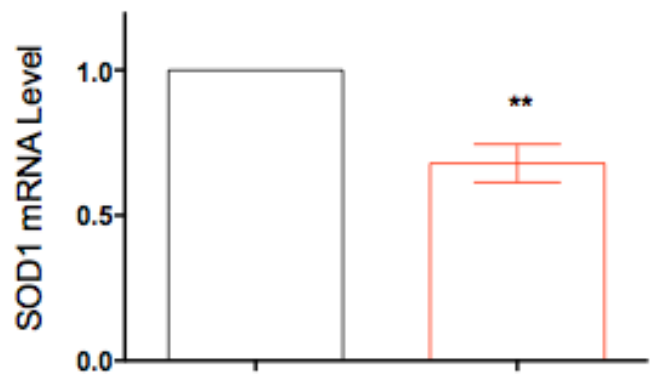

HCT116 (MLH1-VE)

HCT116+chr3 (MLH1+VE)

Figure 4.7. Decreased gene expression of antioxidant defense enzymes in MLH1-deficient cell lines.

(A-D) qRT-PCR was used to measure the expression of several genes in the antioxidant system in the MLH1-deficient cell line HCT116 compared to its isogenetically matched MLH1-proficient cell line HCT116+chr3 Decreased expression of (A) NRF2, (B) catalase, (C) GPX1 and (D) SOD1 was observed in the MLH1-deficient cell lines. Results shown here are from three independent experiments carried out in triplicate. Error bars represent standard error of the mean. 
Figure 4.8

A
HCT116 HCT116+chr3
(MLH1-VE) (MLH1+VE)

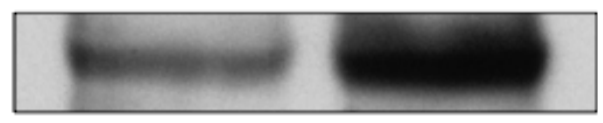

NRF2

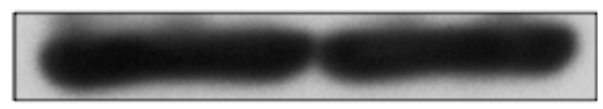

$\beta$-actin

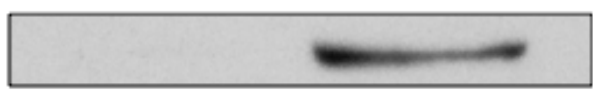

MLH1

1

2.1

C

HCT116 HCT116+chr3

(MLH1-VE) (MLH1+VE)
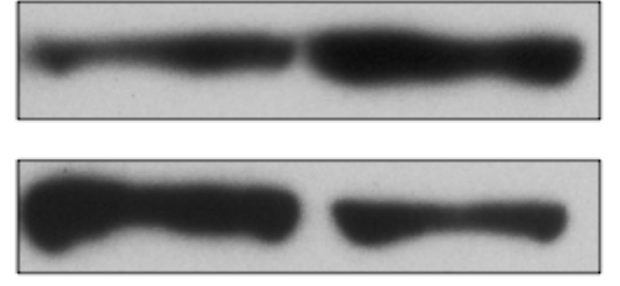

$\beta$-actin

MLH1

Catalase

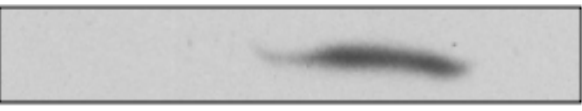

B HCT116 HCT116+chr3 (MLH1-VE) (MLH1+VE)

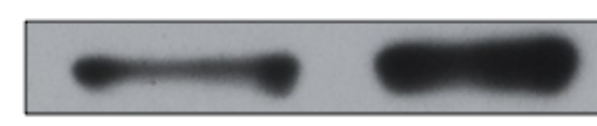

Gpx1

$\beta$-actin

MLH1

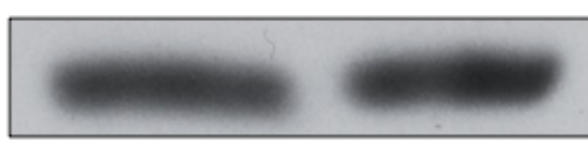

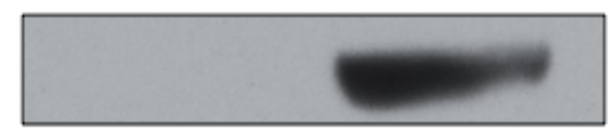

3.1

2.1 
Figure 4.8. Decreased protein levels of antioxidant defense enzymes.

(A-C) Western blot analysis to investigate expression of several antioxidant enzymes in the absence of MLH1 expression. Protein lysates were isolated from the MLH1-deficient HCT116 and the MLH1-proficient HCT116+chr3 cell lines and analyzed by western blotting using an anti-NRF2, anti-catalase or an anti-Gpx1, in addition to MLH1 and $\beta$-actin primary antibodies. Decreased expression of the transcription factors (A) NRF2 as well as the downstream antioxidant enzymes (B) Gpx1 and (C) catalase was observed in the MLH1deficient cell lines. Western blots shown here are one representative example of three independent experiments. Quantification was carried out using ImageJ and represents the fold change in protein level (normalized to $\beta$-actin) in relation to the MLH1-deficient cell line HT116. 


\subsubsection{Investigating Complex I in MLH1 deficiency}

\subsubsection{Decreased Complex I expression in MLH1-deficient cell lines}

Thus far I observed a difference in ROS metabolism and the antioxidant response between MLH1-deficient and proficient cell lines but to get a full understanding of why these differences may be present it was imperative to investigate mitochondrial function since this is where most ROS is produced.

To investigate mitochondrial function, we initially investigated the OXPHOS system since this is one of the main functions of the mitochondria. We isolated protein from our MLH1-deficient and MLH1-proficient cells and performed western blotting to determine the expression of the five respiratory chain complexes. Our data showed decreased expression of Complex I in the MLH1-deficient HCT116 cell line compared to its matched MLH1-proficient cell line HCT116+chr3, using an antibody cocktail targeting all five membrane-bound complexes of the respiratory chain (Figure 4.9A). We validated our observation using the primary antibody NDUFB8 (Figure 4.9B), which detects an accessory subunit of Complex $\mathrm{I}$ and observed decreased NDUFB8 expression. Furthermore we also analysed NDUFB8 expression in our panel of genetically diverse MLH1-proficient and deficient cell lines, however we did not observe consistent decreased NDUFB8 in our MLH1-deficient cells (Figure 4.10A) across this panel. This may be due to the fact that this is a panel of isogenetically diverse cell lines with potentially a range of other mutations that could affect NDUFB8 expression. Another important fact to consider is that Complex I is made up of 45 subunits so there may be mutations in other subunits.

To further investigate deficiencies in Complex I, we analysed gene expression of two mitochondrial-encoded core Complex I subunits MTND1 and MTND2 (Figure 4.11A4.11B) as well as a further nuclear encoded accessory subunit NDUFA (Figure 4.11C) in the MLH1-deficient cell line HCT116 compared to the MLH1-proficient cell line HCT116+chr3 Our data suggests that MTND1, MTND2 and NDUFA expression was significantly decreased in MLH1-deficient cells. 


\section{Figure 4.9}

A
HCT116
(MLH1-VE)
HCT $116+$ chr 3
(MLH1+VE)

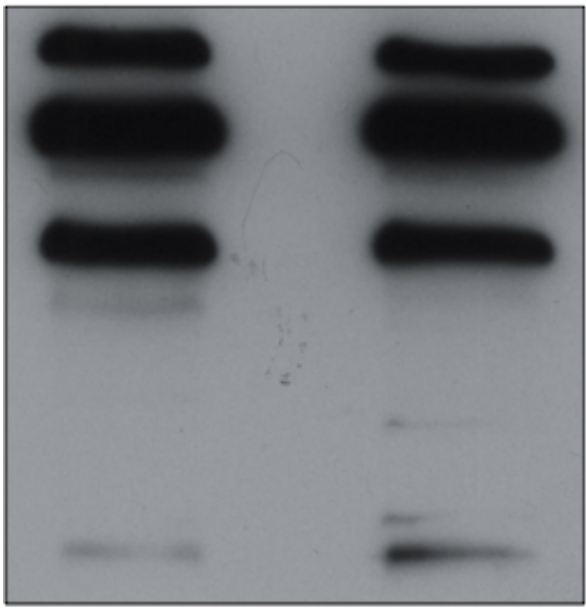

CV

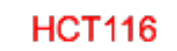

(MLH1-VE)

HCT116+chr3

(MLH1+VE)

CIII

CII

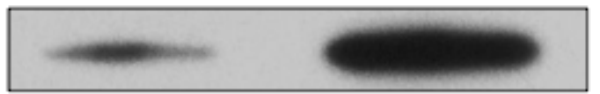

NDUFB8

1

6.3

CIV

Cl

$\beta$-actin

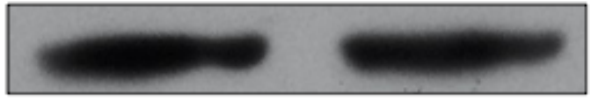

$\beta$-actin

MLH1

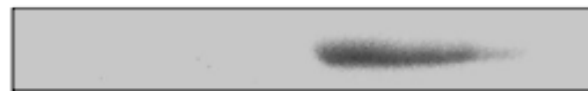

MLH1

Figure 4.9. Decreased expression of Complex I in MLH1-deficient cell lines.

(A-B) Western blot analysis to investigate expression of Complex 1 in the absence of MLH1. Protein lysates were isolated from the MLH1-deficient cell line HCT116 and the MLH1proficient cell line HCT116+chr3 cell and analyzed by western blotting using (A) anti-OXPHOS (cocktail antibody detecting all five oxidative phosphorylation complexes) and (B) anti-NDUFB8 (accessory subunit of Complex I), in addition to MLH1 and $\beta$-actin primary antibodies. (A-B) Quantification was carried out using ImageJ and represents the fold change in protein level (normalized to $\beta$-actin) in relation to the MLH1-deficient cell line HT116. 


\section{Figure 4.10}

A

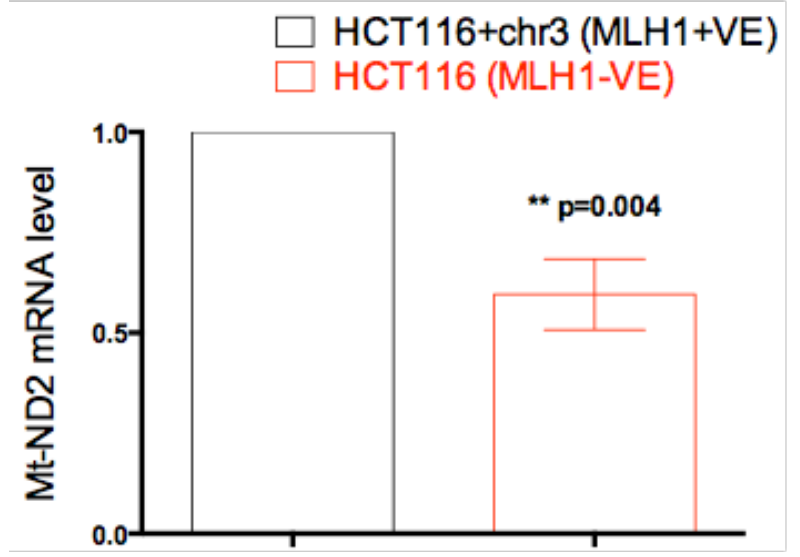

C

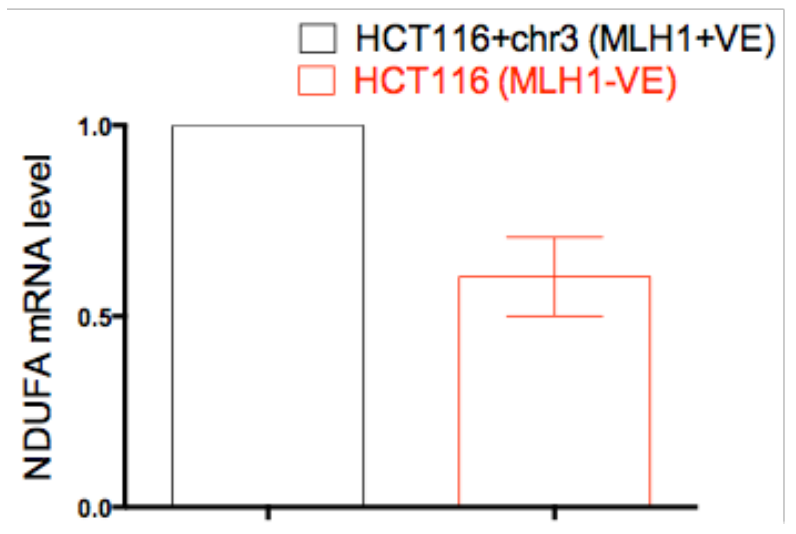

B

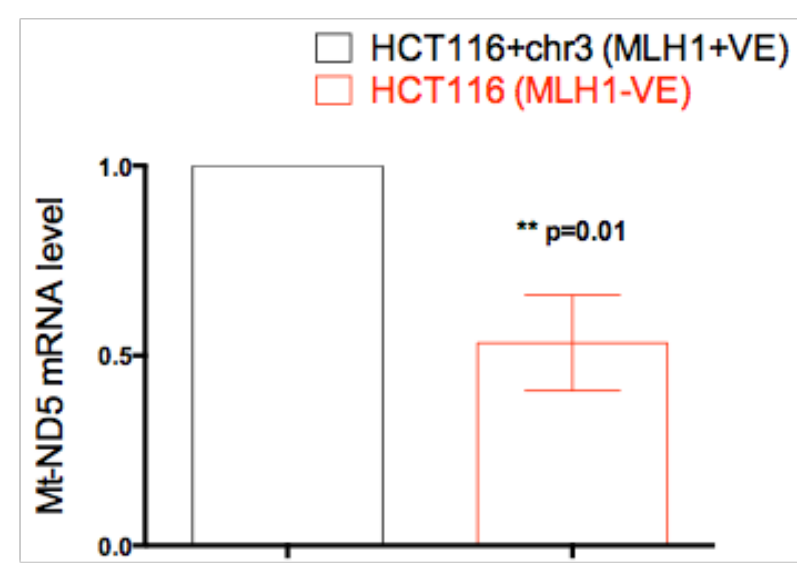

Figure 4.10. Decreased expression of mitochondrial encoded Complex I subunits in MLH1deficient cell lines.

(A-B) qRT-PCR was used to measure the expression of several mitochondrial encoded Complex I genes in the MLH1-deficient cell line HCT116 compared to its isogenetically matched MLH1proficient cell line HCT116+chr3 Decreased expression of (A) Mt-ND2, (B) Mt-ND5, (C) NDUFA was observed in the MLH1-deficient cell lines. 
Figure 4.11
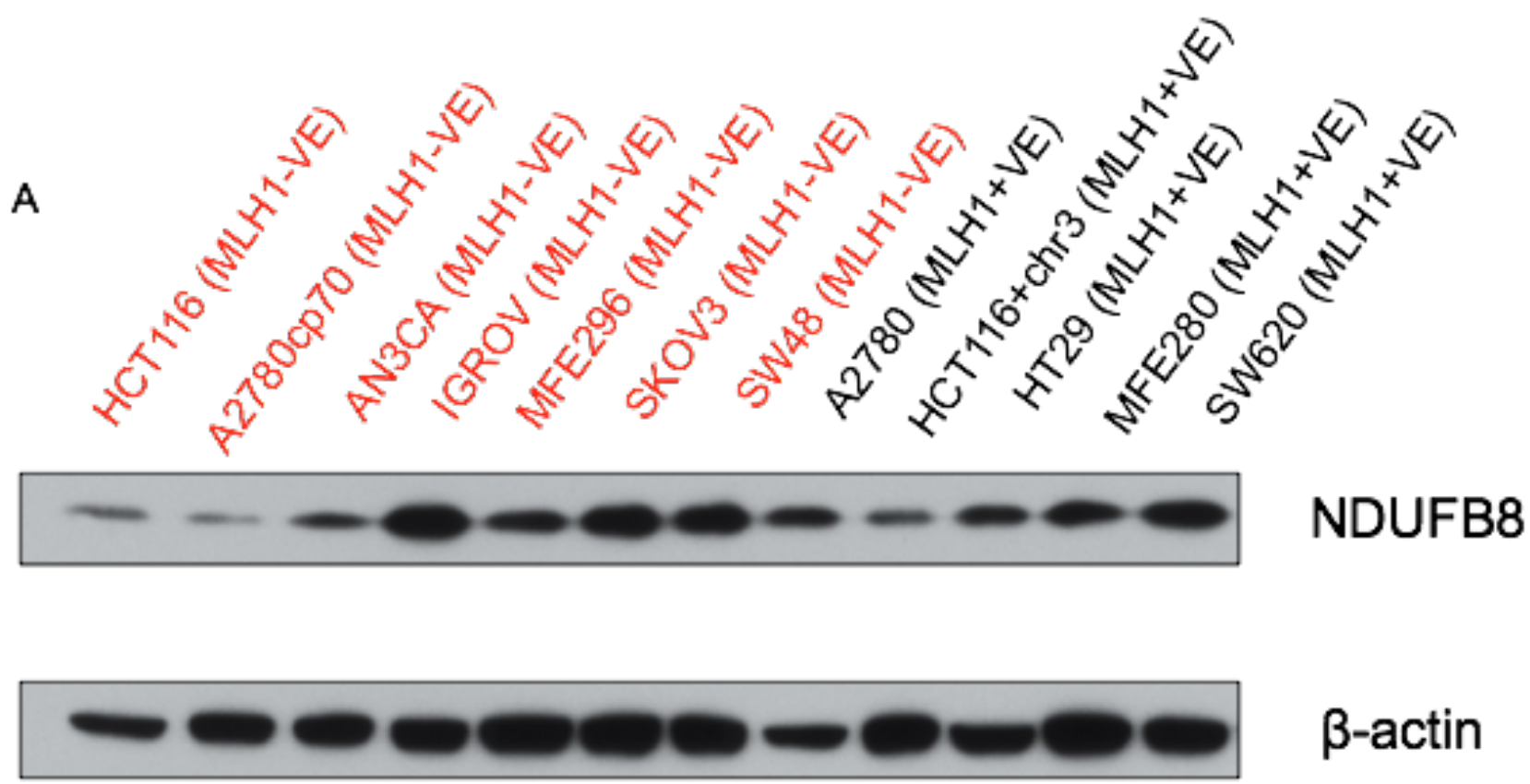

$\beta$-actin

\section{MLH1}

Figure 4.11. Complex I expression is not consistently decreased in a panel of MLH1-deficient cell lines.

(A) Western blot analysis to investigate expression of Complex $\mathrm{I}$ in the absence of MLH1. Protein lysates were isolated from the MLH1-deficient cell lines cell lines HCT116, SKOV3, IGROV, MFE-296, A2780cp70, AN3CA, SW48 and the MLH1-proficient cell lines HCT116+chr3, A2780, SW620, MFE280 and HT29 and analyzed by western blotting using antiNDUFB8 (accessory subunit of Complex I), in addition to MLH1 and $\beta$-actin primary antibodies. 


\subsubsection{Decreased activity of Complex I in MLH1-deficient cell lines}

Given that the panel of cell lines have a diverse genetic makeup, aside from their differences in MLH1 status and the fact that Complex I is made up of multiple subunits, we decided to measure Complex I activity since this may be more biologically relevant. Complex I activity was measured using an ELISA assay assessing the oxidation of $\mathrm{NADH}$ to $\mathrm{NAD+}$, which in turn leads to reduction of a dye and increased absorbance at OD $450 \mathrm{~nm}$. We observed a significant decrease in the activity of Complex I in the MLH1-deficient cell line HCT116, compared to the MLH1-proficient HCT116+chr3 cells (Figure 4.12A). We also observed a statistically significant decrease in the activity of Complex I in our panel of MLH1-deficient cell lines compared to the MLH1-proficient cell lines. (Figure 4.12B). We hypothesized from these results that the decrease in Complex I activity in the absence of MLH1, may have a role to play in the selectivity we observe with Parthenolide.

Given that I had observed the selectivity of MLH1 with Parthenolide in a panel of cell lines I also looked at the expression of NDUFB8 in this panel of cell lines to see whether deficiencies in Complex I might explain the mechanism of selectivity. I did not see a similar trend in my panel of genetically diverse MLH1-deficient and proficient cell lines (Figure 4.11). However when I examined Complex I activity which is more biologically relevant, a significant difference in Complex I expression became apparent between the MLH1-deficient and proficient cell lines in my panel of cells (Figure 4.12B)

Given how integral Complex I is to oxidative phosphorylation, I hypothesized that my MLH1-deficient cell lines may therefore also have deficiencies in oxidative phosphorylation . 
Figure 4.12

A

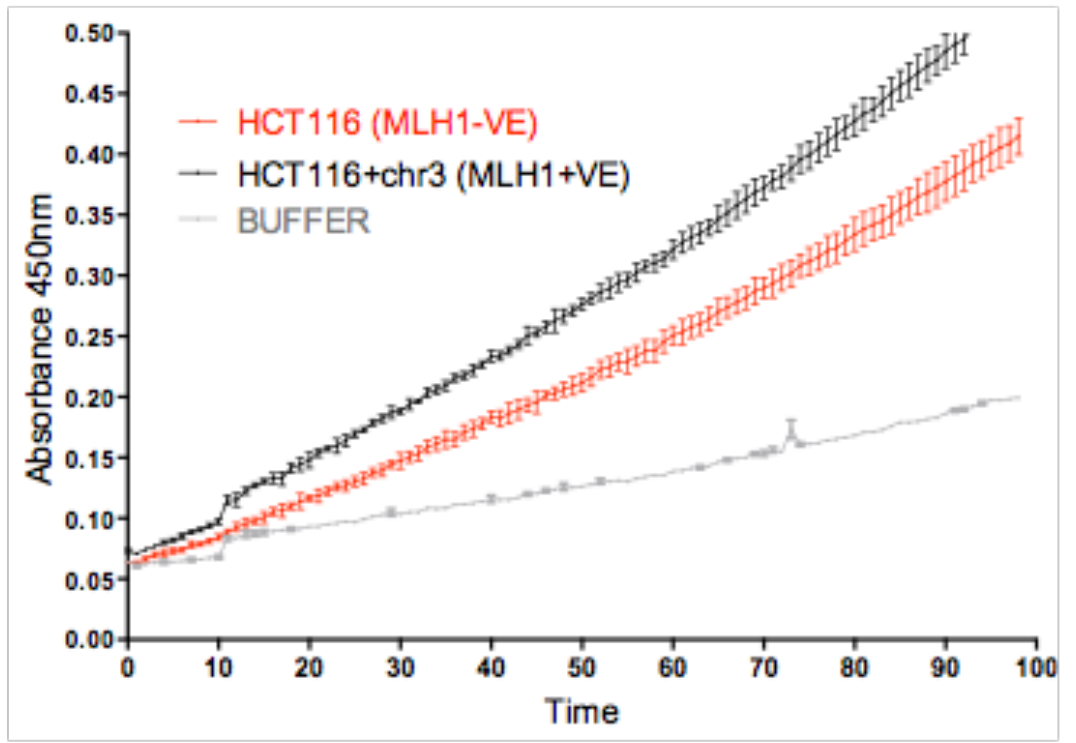

B

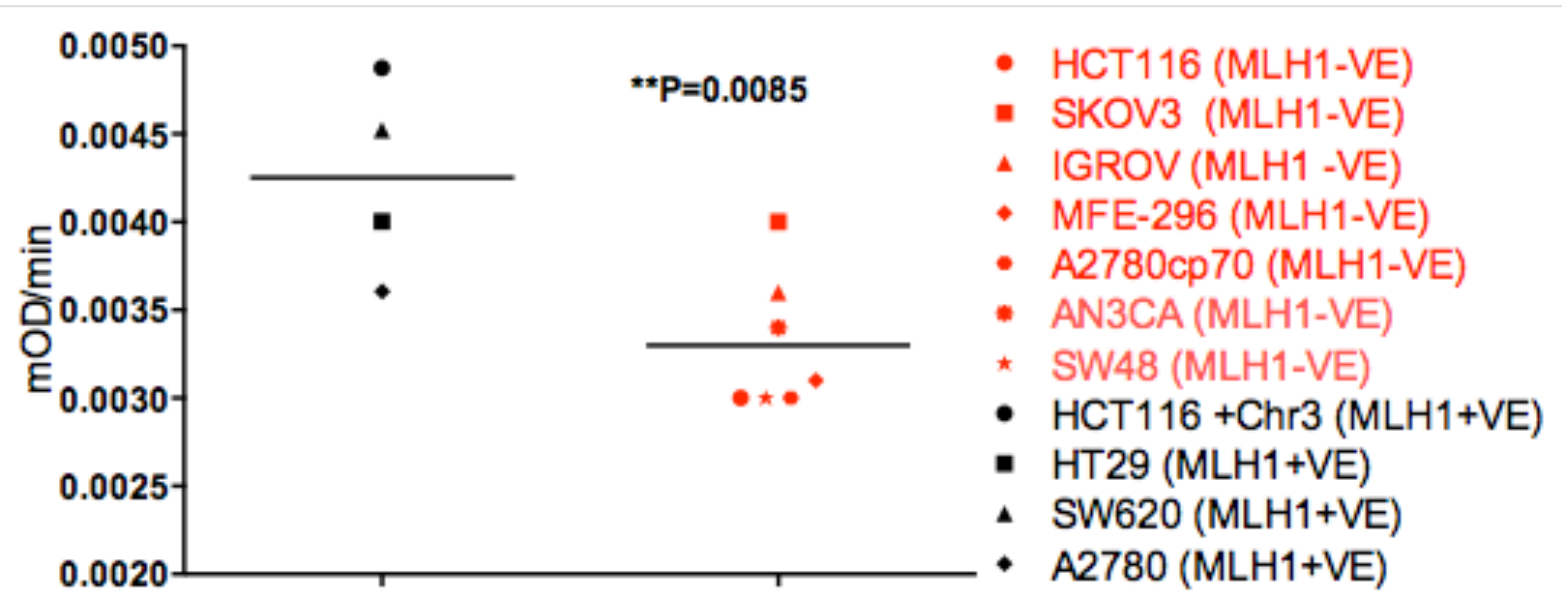


Figure 4.12. Decreased Complex I activity in MLH1-deficient cell lines.

(A-B) An ELISA assay was used to measure Complex I activity. Protein lysates were isolated and quantified and equal amounts of protein were incubated in the ELISA plate to determine the activity of Complex I by measuring the oxidation of $\mathrm{NADH}$ to NAD+ and the simultaneous reduction of a dye leading to increased absorbance at $450 \mathrm{~nm}$, over time. (A) Complex I activity was measured using the ELISA described with protein lysates from the MLH1-deficient HCT116 and MLH1-proficient HCT116+Chr3 cell lines. (B)The activity of Complex I was determined in the panel of MLH1-deficient cell lines (HCT116, SKOV3, IGROV, AN3CA,MFE-296, SW48, A2780cp70) and the MLH1-proficient cell lines (HCT116+chr3, HT29,SW620, A2780) by using the average of duplicates performed in three independent experiments. (A) The graph shown here is a representation of the consistent difference seen in three experiments done in triplicate samples. (B) The graph shown here is the average of three experiments carried out in triplicate samples 


\subsubsection{Investigating Oxidative phosphorylation upon MLH1 deficiency}

\subsubsection{MLH1-deficient cell lines have decreased oxidative phosphorylation}

Thus far, we identified significant differences in the expression and activity of Complex I in MLH1-proficient and -deficient cell lines. Given that Complex I is the biggest Complex In the oxidative phosphorylation chain, we wanted to examine whether there was a difference in oxidative phosphorylation and mitochondrial metabolism in general between the MLH1-deficient and -proficient cell lines using the Seahorse XF analyzer. The $\mathrm{XF}$ analyzer measures the rate of oxygen consumption in a given sample providing a measure of oxidative phosphorylation. The basal oxidative consumption rate (OCR) when no drugs are added to the sample allows a measure of basal respiration. The addition of the uncoupling agent FCCP provided a measure of the cells maximal respiratory capacity and when the basal OCR is taken away from the maximal respiration a measure of spare respiratory capacity (SRC) is obtained. The XF analyzer was used to measure the difference in the OCR as a measure of oxidative phosphorylation in the HCT116 and HCT116+chr3 cell lines as well as in a panel of MLH1-proficient and -deficient cell lines. Our experiments have shown a significant decrease in the basal OCR (Figure 4.13A+4.13B) and a decrease in the SRC (Figure 4.13C) in the MLH1-deficient HCT116 cells compared to the MLH1-proficient HCT116+chr3 cells. We also observed a statistically significant decrease in the basal OCR in the panel of MLH1-deficient cell lines (figure 4.13D), compared to the MLH1proficient cell lines. These results confirm that not only is Complex I deregulated in MLH1-deficient cells; there is also decreased oxidative phosphorylation in general, in the absence of MLH1 expression. 
Figure 4.13

A

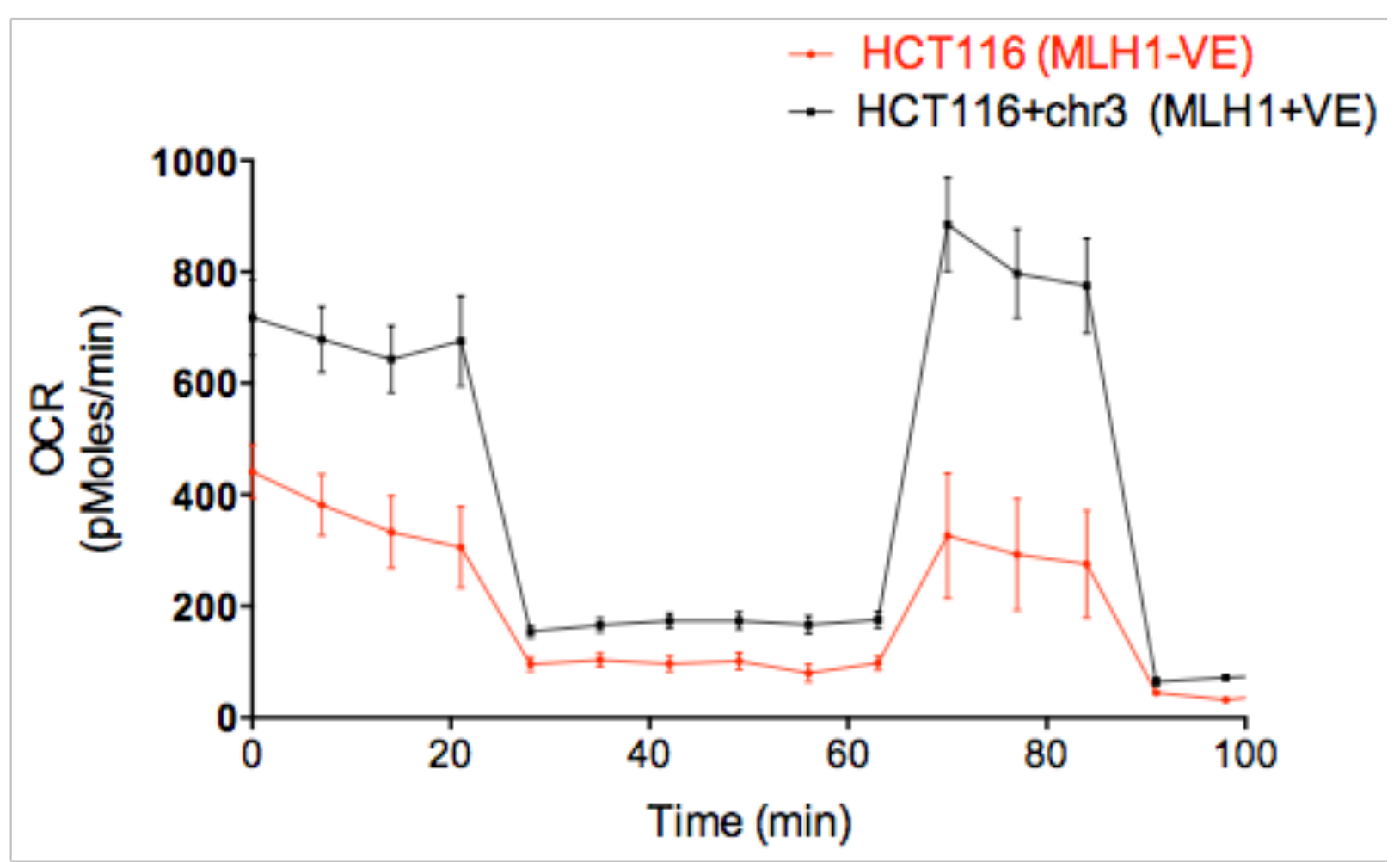

B

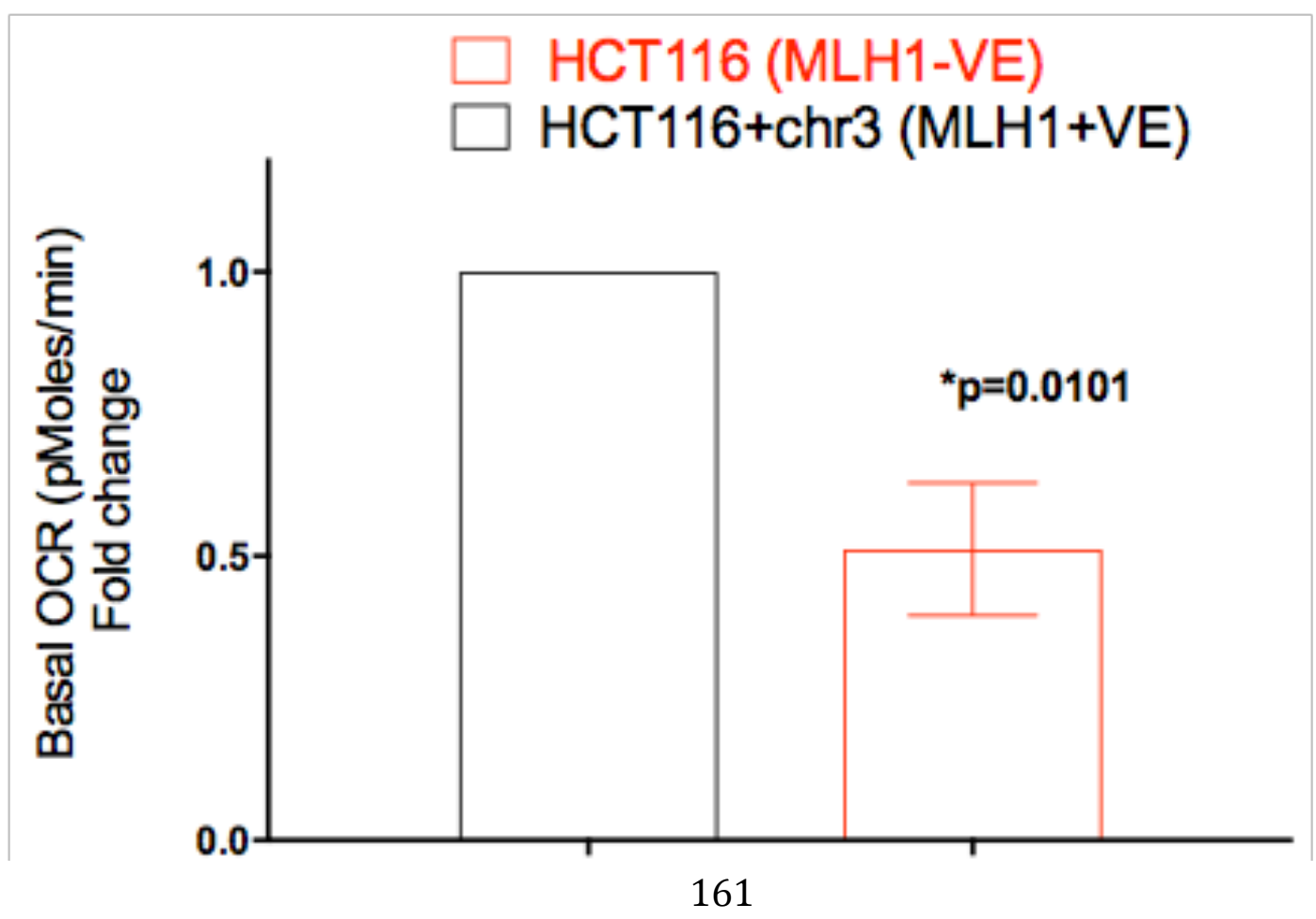


C

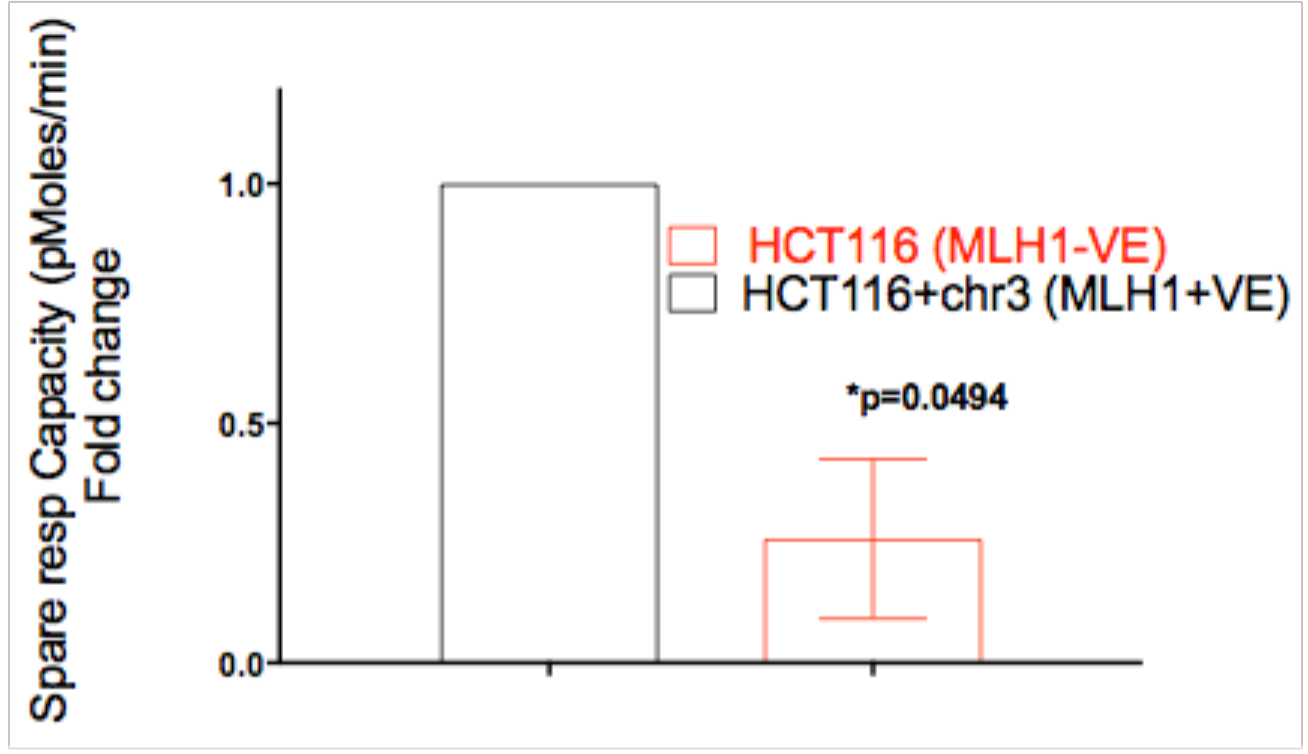

D

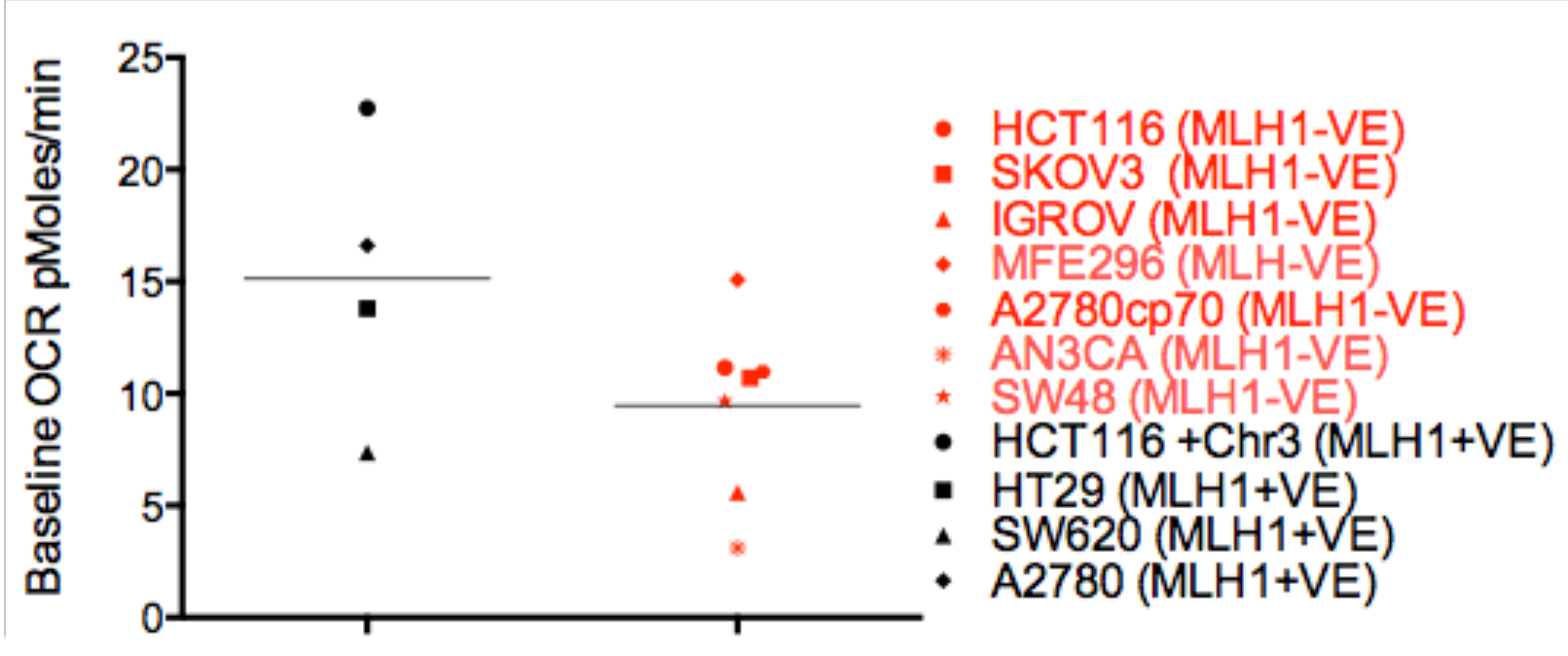


Figure 4.13. Reduced basal OCR and spare respiratory capacity in MLH1-deficient cell lines. (A-D) The XF analyzer was used to analyze the OCR as a measure of oxidative phosphorylation. The basal OCR was determined prior to the addition of drugs to ascertain the full bioenergetic profile. The addition of Oligomycin (1um) inhibits complex $V$ and approximates leak of the inner mitochondrial membrane. The addition of FCCP (0.25um) allows for measurement of maximal respiration. Mitochondrial respiration is then inhibited by the addition of the Complex I inhibitor Rotenone (1um) and the Complex III inhibitor Antimycin (1um) to ascertain non-mitochondrial sources of oxygen consumption. (A) An example illustrating the results of the full bioenergetic profile from one experiment using the MLH1 -deficient HCT116 cell line and the MLH1-proficient HCT116+chr3 cell line. (B) The basal OCR and (C) spare respiratory capacity was significantly decreased in the MLH1 -deficient cell line HCT116, compared to the MLH1-proficient cell line HCT116+chr3 (D) The basal OCR was lower in a panel of MLH1-deficient cell lines (HCT116, SKOV3, IGROV, AN3CA, MFE-296, SW48, A2780cp70) compared to the MLH1-proficient cell lines (HCT116+chr3,HT29,SW620, A2780). (B-D) Results of three experiments done in triplicate are shown here and error bars represent standard error of the mean. 


\subsubsection{Investigating differences in the mitochondrial genome between MLH1- proficient and deficient cell lines}

4.2.4.1 There are no significant differences in mutations within Complex I genes in MLH1-deficient and proficient cell lines

To explore the reasons behind the deficiencies in Complex I that I observed I hypothesized that there may be an increased number of mutations within microsatellites in mitochondrial encoded Complex I genes between MLH1-deficient and proficient cell lines. Increased mutations within microsatellites, due to the nature of these repetitive sequences has been extensively described in CRC with MMR deficiency. [374, 375]. Similar observations of MSI in the mitochondrial genome have been suggested but this concept has not been as well established as MSI in the nuclear genome. One study by Habano et al discovered that out of 45 sporadic colorectal cancers, $44 \%$ had mutations within microsatellites in the non-coding D-loop region of the mtDNA and tumours also had mutations within mitochondrial encoded Complex I genes [76]. This study was the first indication that MMR deficient tumours may harbour mutations within Complex I genes. More recently, Mishra et al proved that MLH1 deficiency (as a result of retinal endothelial cells exposed to high glucose) leads to increased sequence variants in the mitochondrial D-loop region and decreased OCR. All of these finding were ameliorated when MLH1 was overexpressed [78].

To investigate if MSI may account for the deficiencies in Complex I that I had observed, I carried out experiments using the Illumina MiSeq platform to carry out next generation sequencing on the mitochondrial genome of the isogenetically matched cell lines HCT116 which is MLH1-deficient and HCT116+chr3 which is MLH1-proficient. Interestingly I did not observe a difference in mutations within the seven known mitochondrial encoded Complex I genes as well as the non-coding D-loop regions which is known to harbour mutations affecting Complex I (Appendix 1). 


\subsubsection{Investigating mitochondrial biogenesis in MLH1-deficient cell lines}

\subsubsection{Decreased mitochondrial biogenesis in MLH1-deficient cell lines.}

My results thus far have displayed a mitochondrial phenotype with a deregulated antioxidant response, deficiencies in Complex $\mathrm{I}$ and decreased oxidative phosphorylation in MLH1-deficient cell lines. Therefore I next investigated the possible reasons to explain this. We initially examined the expression of the nuclear transcriptional co-activator PGC-1 $\beta$ in my MLH1-deficient and proficient cell lines since this co-activator is known as the master regulator of mitochondrial biogenesis and has been described to mediate the transcription of a multitude of nuclear encoded mitochondrial genes [325]. Significantly, we observed a decrease in the expression of PGC-1 $\beta$ (Figure 4.14A) in our MLH1-deficient cell line compared to the isogenetically matched MLH1-proficient cell line HCT116+chr3 suggesting that the MLH1-deficient cell lines were likely to have decreased mitochondrial biogenesis compared to the MLH1proficient cell lines. I also analysed the expression of NRF1, which is downstream of PGC-1 $\beta$ and co-activates mitochondrial transcription factor $A$, which in turn regulates mitochondrial biogenesis and function [333]. In concurrence with our previous results, NRF1 protein levels (Figure 4.14B) and gene expression (Figure 4.14C). was reduced in the MLH1-deficient cell line HCT116 compared to the MLH1 proficient cell line HCT116+chr3. I also carried out qPCR experiments to determine the mitochondrial copy number of my samples by determining the ratio of the expression of mitochondrial tRNA to nuclear $\beta 2 M$. There was a striking decrease in the mitochondrial copy number of the MLH1-deficient HCT116 cell line compared to proficient cell line HCT116+chr3 (Figure 4.14D). Both these findings demonstrate that MLH1-deficient cell lines have decreased mitochondrial biogenesis and taken together with my other findings in this study, this is likely to lead to deficiencies in Complex I and oxidative phosphorylation. 
Figure 4.14

A

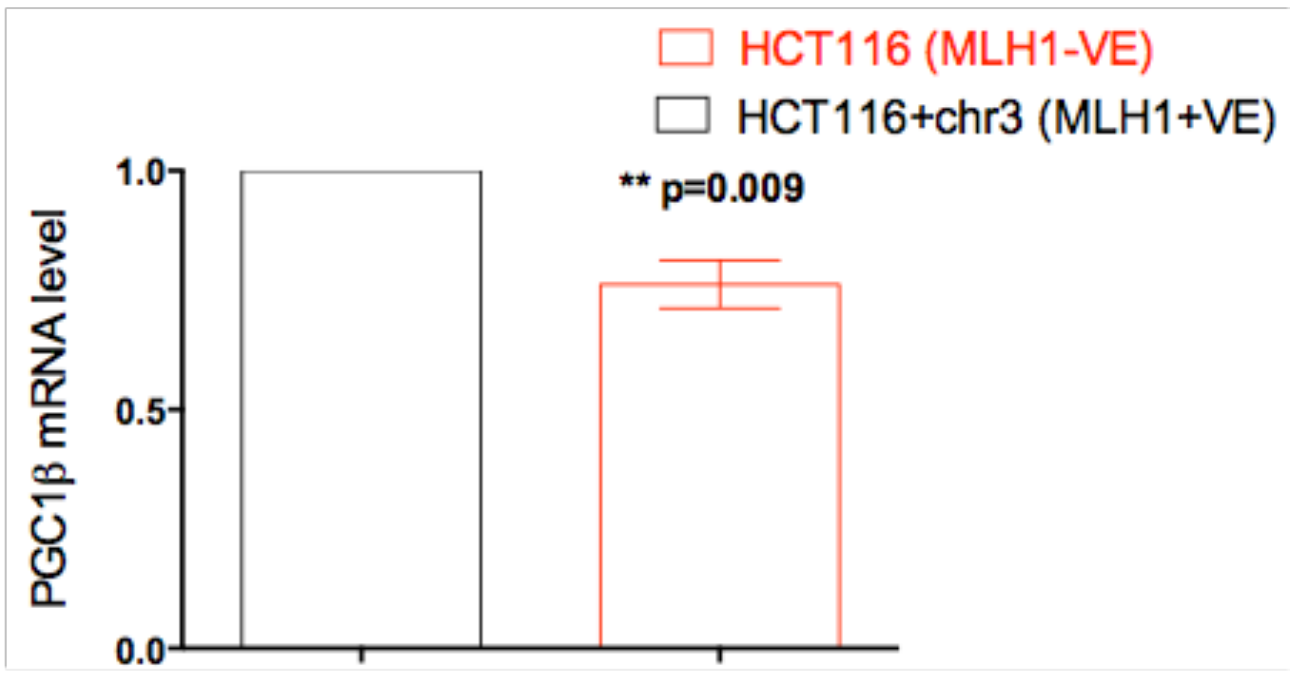

B

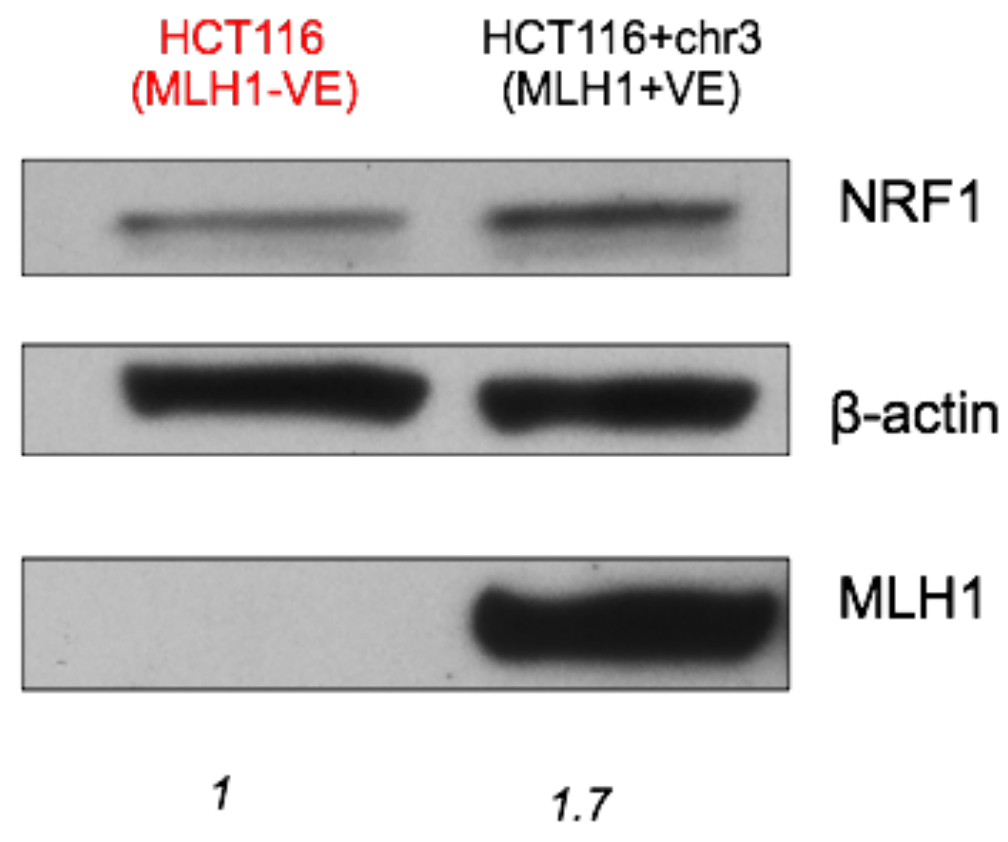


C

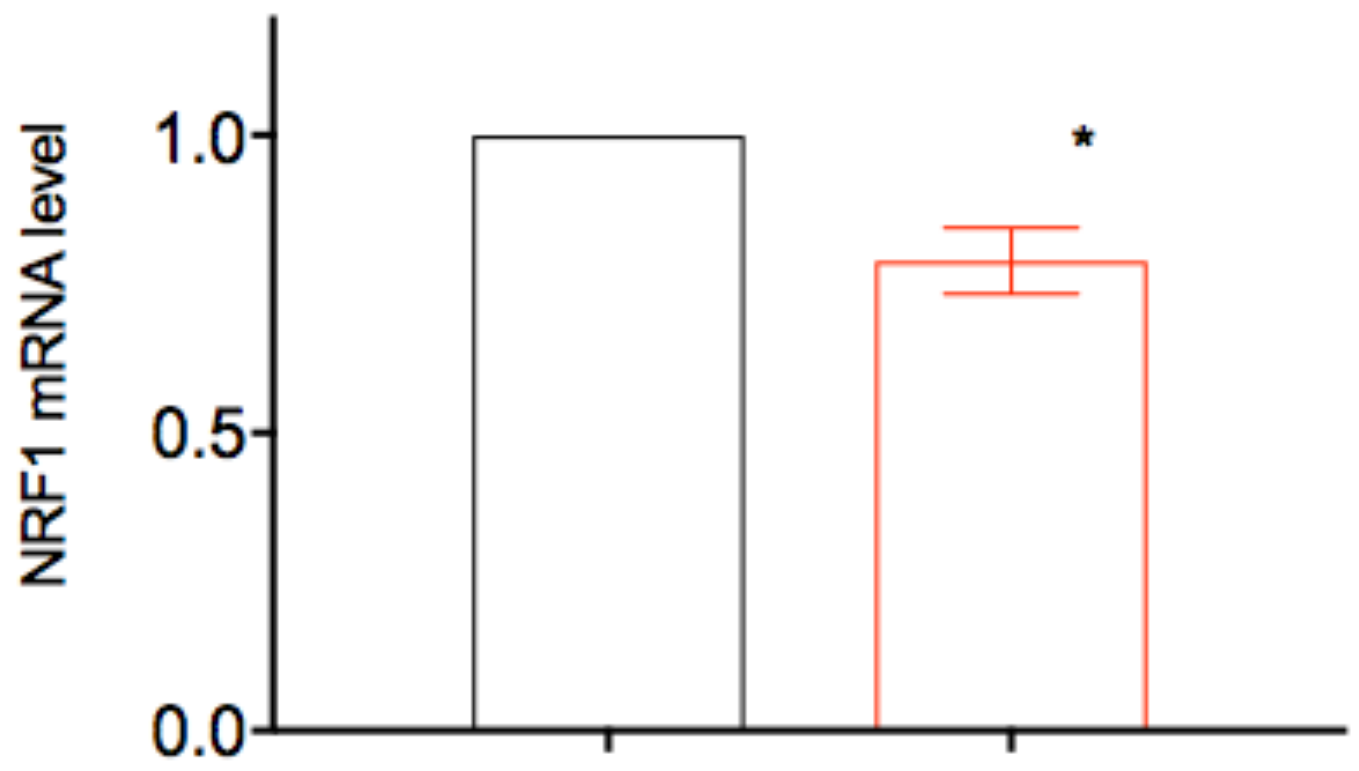

D

- HCT116 (MLH1-VE)

- HCT116+chr3 (MLH1+VE)

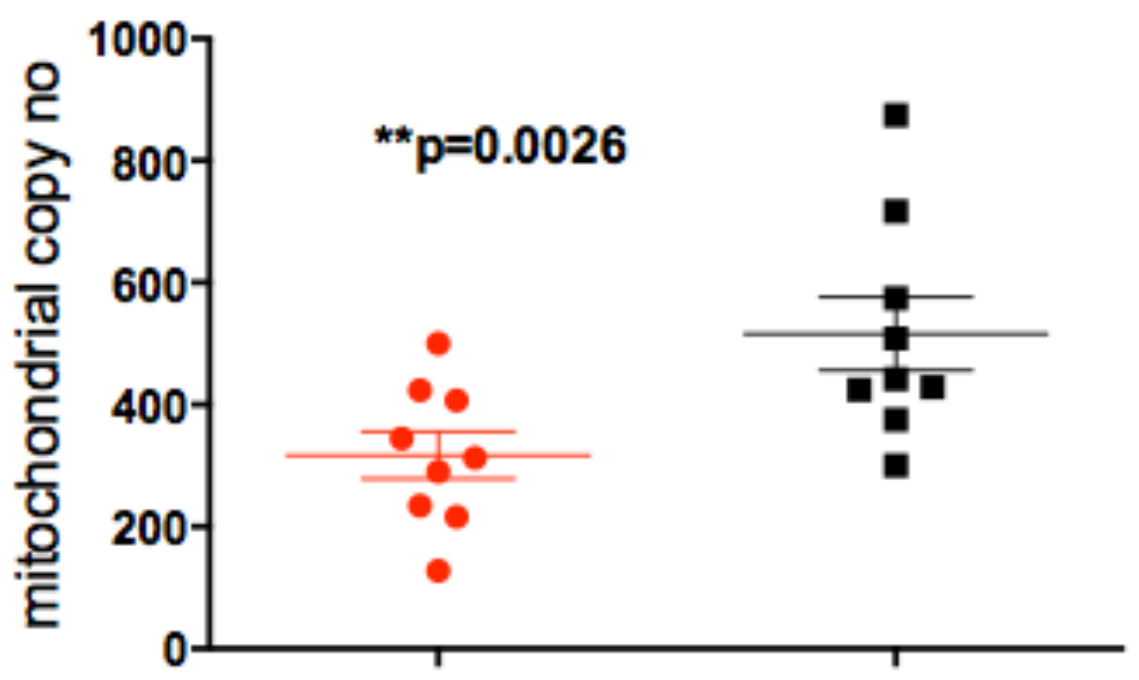


Figure 4.14. Reduced mitochondrial biogenesis and mtDNA copy number in MLH1-deficient cell lines. (A) qRT-PCR was used to measure the expression of PGC1 $\beta$. There was decreased expression of pgc1 $\beta$ in the MLH1-deficient cell line HCT116 compared to its isogenetically matched MLH1-proficient cell line HCT116+chr3 (B) Western blot analysis to investigate expression of NRF1 in the absence of MLH1 expression. Protein lysates were isolated from the MLH1-deficient HCT116 and the MLH1-proficient HCT116+chr3 cell lines and analyzed by western blotting using an anti-NRF1, MLH1 and $\beta$-actin primary antibodies. NRF1 expression was significantly decreased in the MLH1 deficient cell line HCT116 compared to the MLH1 proficient cell line HCT116+chr3 (C) qRT-PCR was used to measure the expression of NRF1 in the same cell lines and there was a significant reduction in the gene expression of NRF1 in the MLH1 deficient HCT116 cell line compared to the MLH1 proficient cell line HCT116+chr3 (D) Mitochondrial copy number was also determined in the same cell lines using qPCR with genomic DNA. There was a significant decrease in the average mitochondrial copy number in the HCT116 cell line compare to the MLH1-proficient cell line HCT116+chr3. Results of three independent experiments each with three biological repeats carried out in triplicate are shown here. 


\subsection{Validating experimental results in patient samples}

\subsubsection{Analysis of expression of antioxidant defense enzymes in MLH1-deficient tumours from patient samples}

4.3.1.1 There is no significant decrease in expression of antioxidant defense genes in MLH1-deficient and MSI patient tumour samples

In order to determine whether a similar phenotype may be present in tumours from MLH1-deficient patient samples I decided to examine the gene expression of the antioxidant defense enzymes using qRT-PCR using RNA from MMR-deficient and MMR-proficient patient tumour samples and comparing it to RNA from adjacent normal tissue (for characteristics of patient samples see Table 3.14). I carried this experiment out in tumour samples known to be MSI positive and determined whether they were MLH1 negative by qRT-PCR. Similar experiments were also carried out in MSS tumour samples in order to compare MSI tumours with MSS tumours. I did not see the same decreased expression of the antioxidant defense enzymes (NRF2, SOD1, Catalase and GPX1) or genes involved in mitochondrial biogenesis (PGC1 $\beta$ and NRF1) in the MLH1deficient tumours compared to normal tissue (Figure 4.15A-4.15F). Furthermore there was no significant difference in the fold change (compared to matched normal tissue) in expression of the antioxidant defense enzymes in MSI tumours compared to MSS tumours (Figure 4.16A-4.16F and Figure 4.17).

Upon comparing the expression of $P G C 1 \beta, N R F 1$ and the antioxidant defense genes in the patient samples, I did not observe a consistent difference in expression in these genes between the tumour samples and matched normal tissue in the MLH1-deficient tumours. There was also no significant difference in the expression of these genes between the MSI and MSS tumours. 
Figure 4.15

A

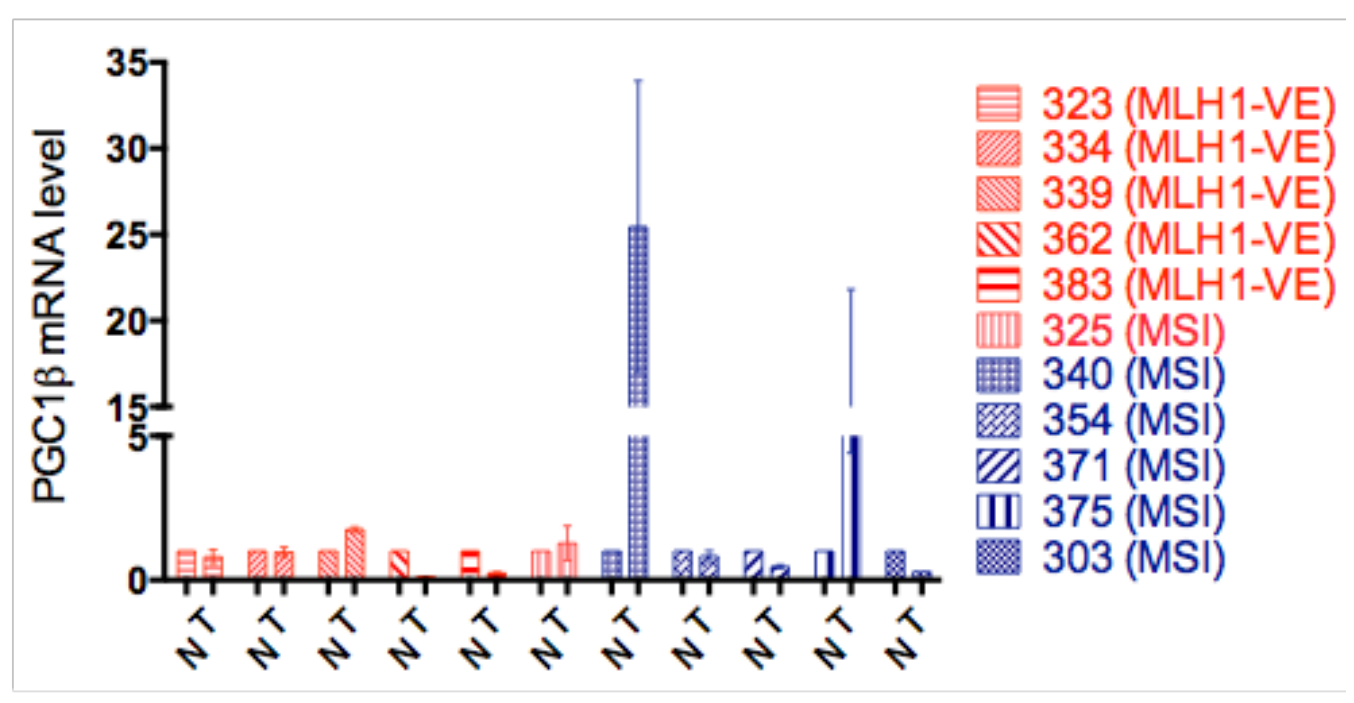

B

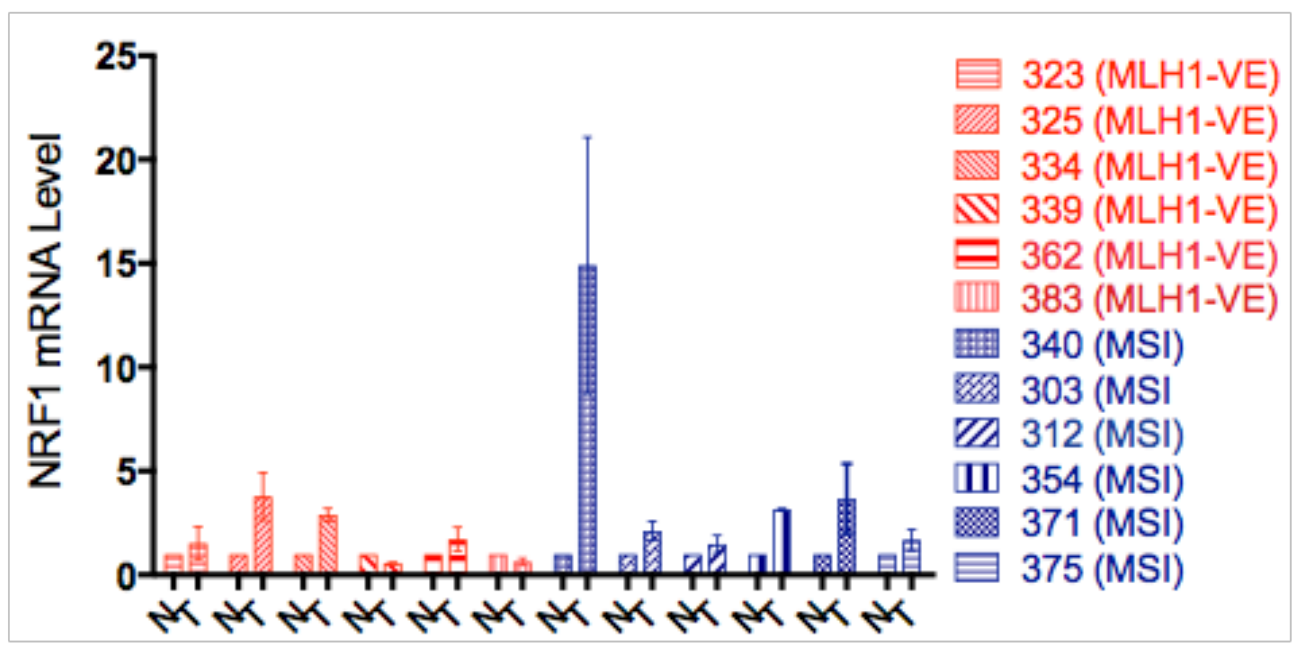

C

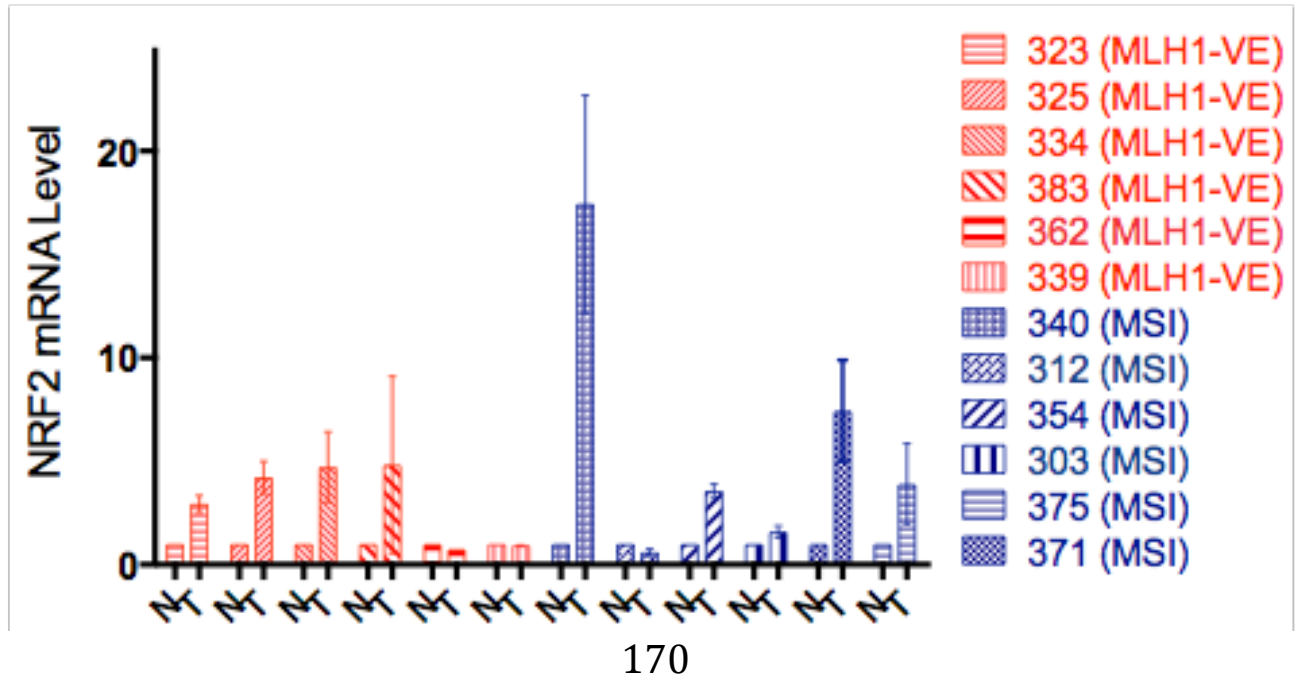


D

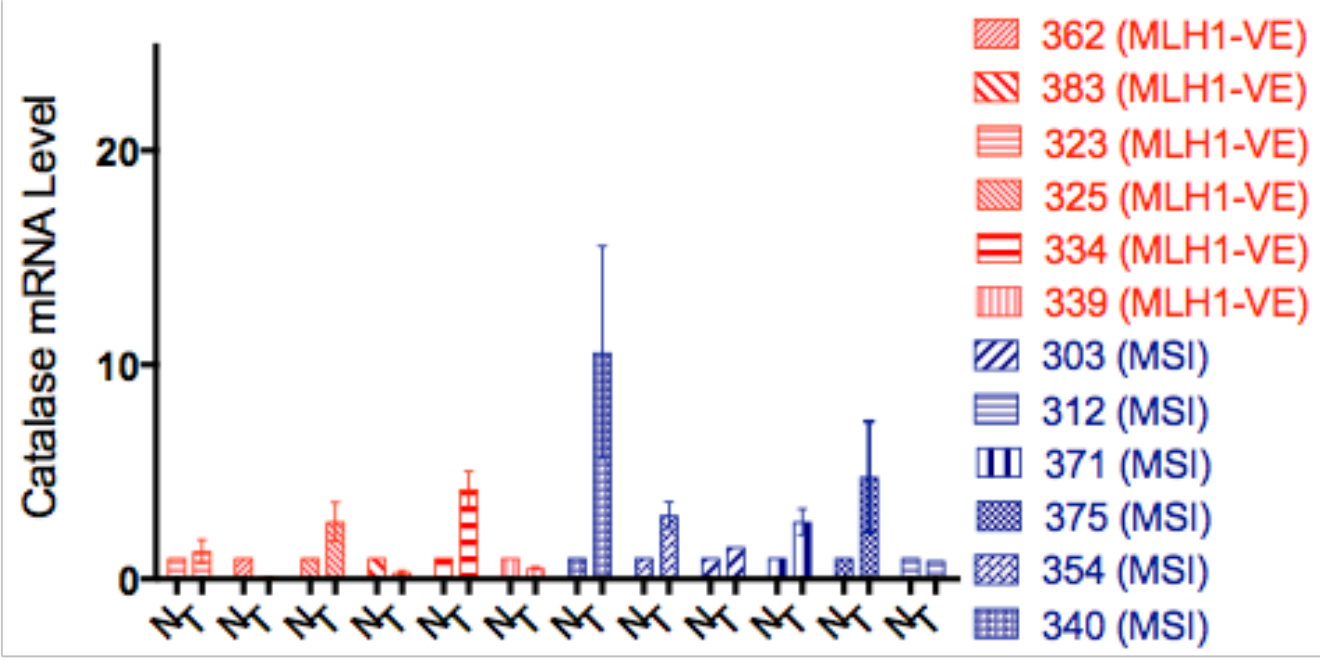

E

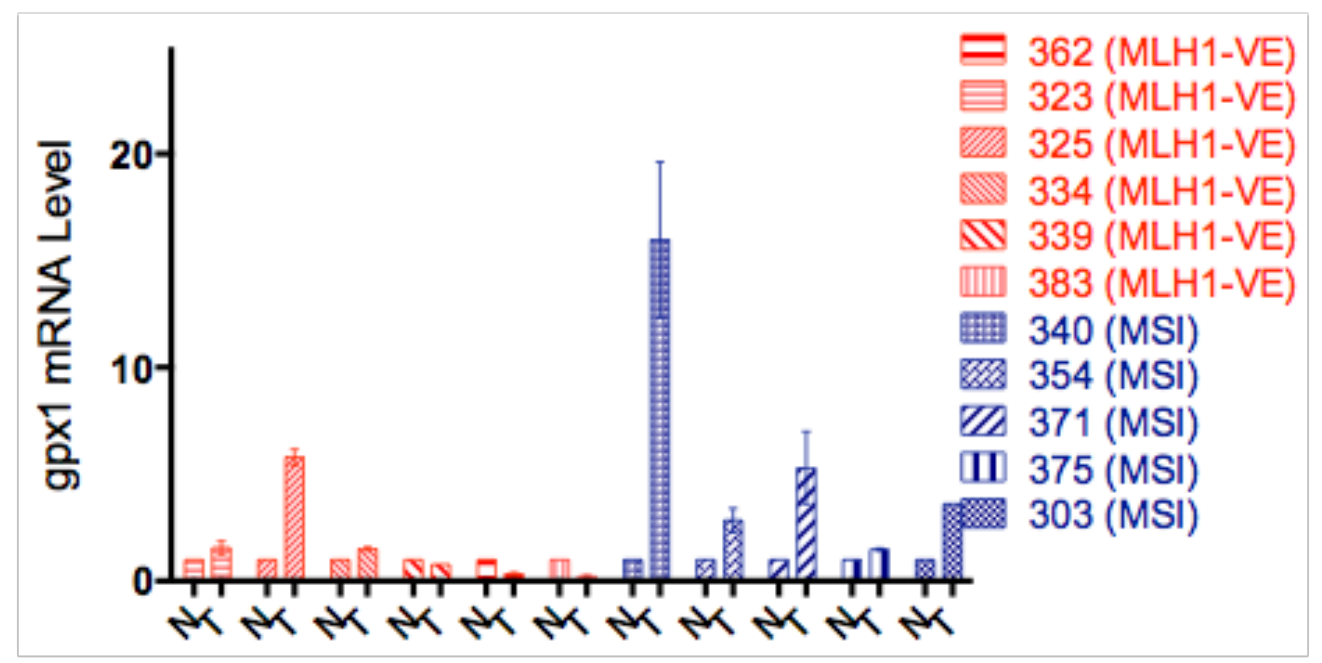

F

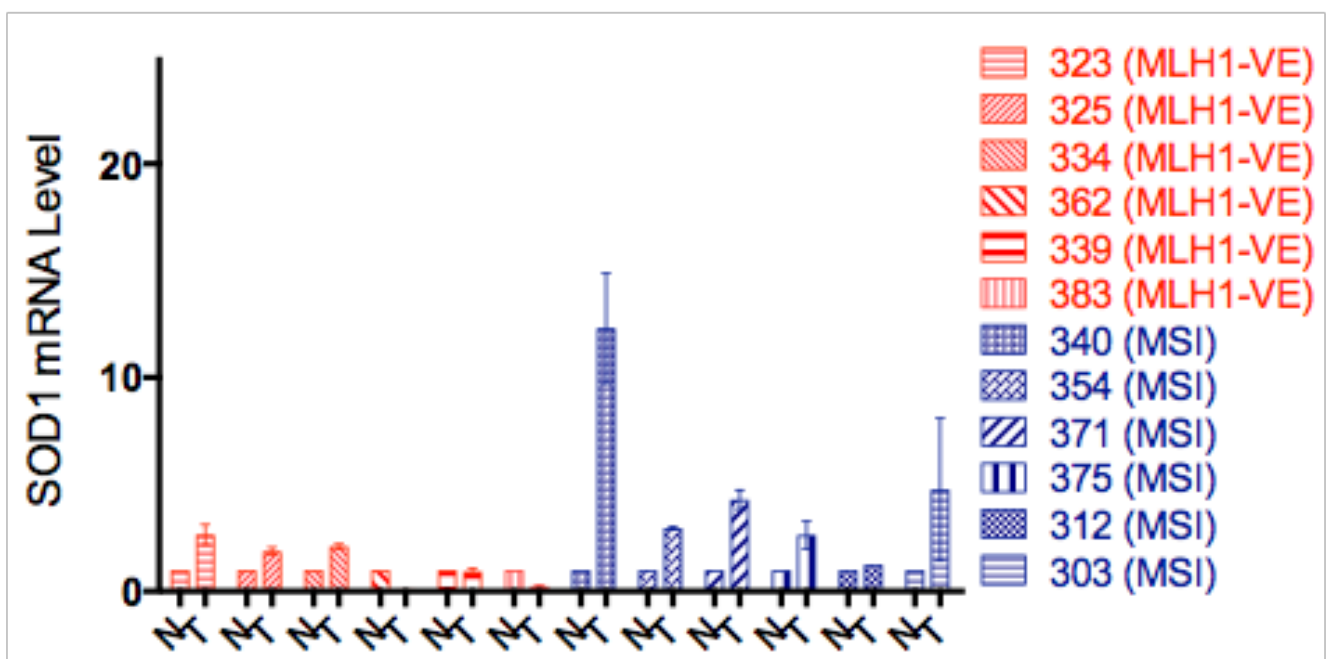


Figure 4.15. MLH1-deficient tumours from patient samples do not exhibit decreased expression of antioxidant defense enzymes compared to surrounding normal tissue.

(A-F) qRT-PCR was used to measure the expression of several genes in the antioxidant system in MSI tumours (MLH1-deficient tumours shown in red) compared to surrounding normal tissue. MSI (and MLH1-deficient) tumours did not show a decreased expression of (A) PGC1 $\beta$, (B) NRF1, (C) NRF2, (D) catalase, (E) GPX1 and (F) SOD1 compared to surrounding normal tissue. Results shown here are from two independent experiments carried out in triplicate. Error bars represent stand error of the mean. 
Figure 4.16

A
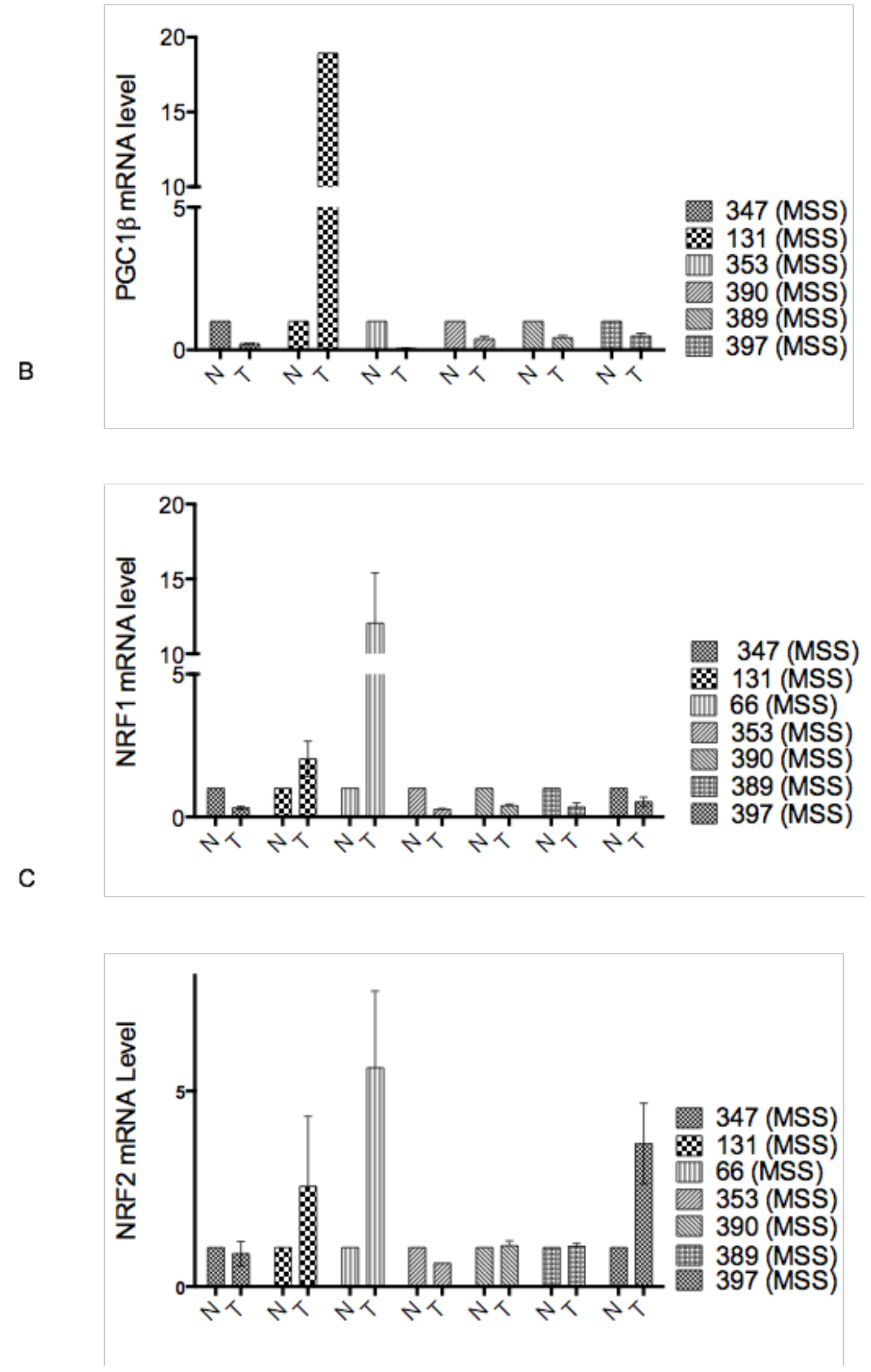
D

E

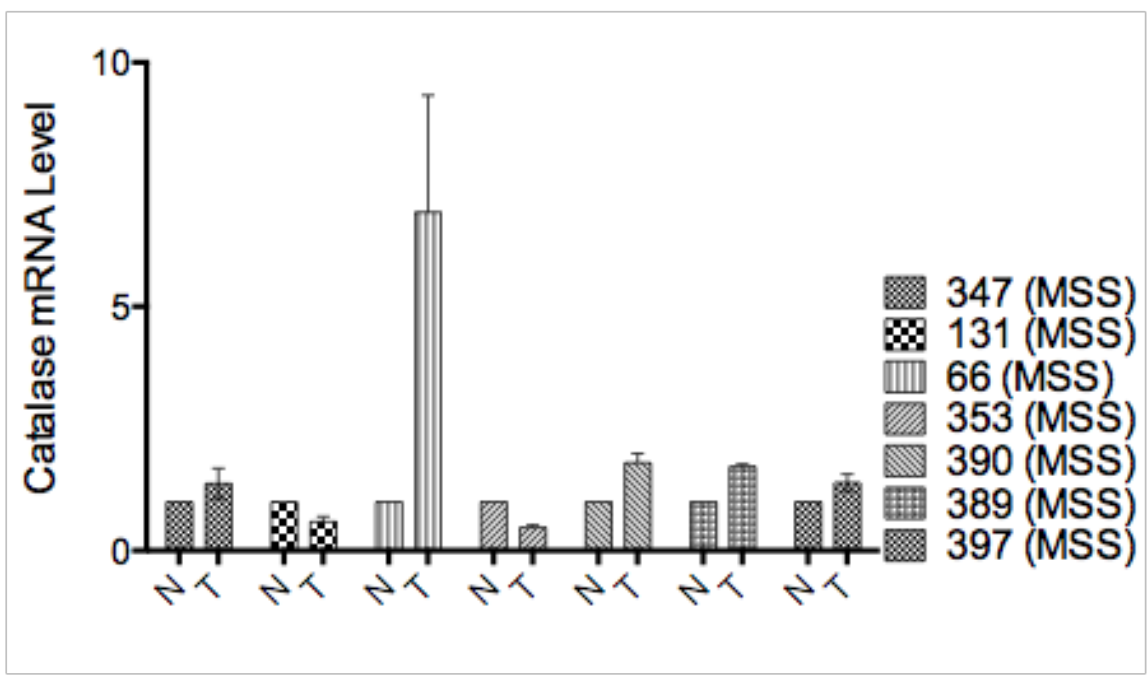

F
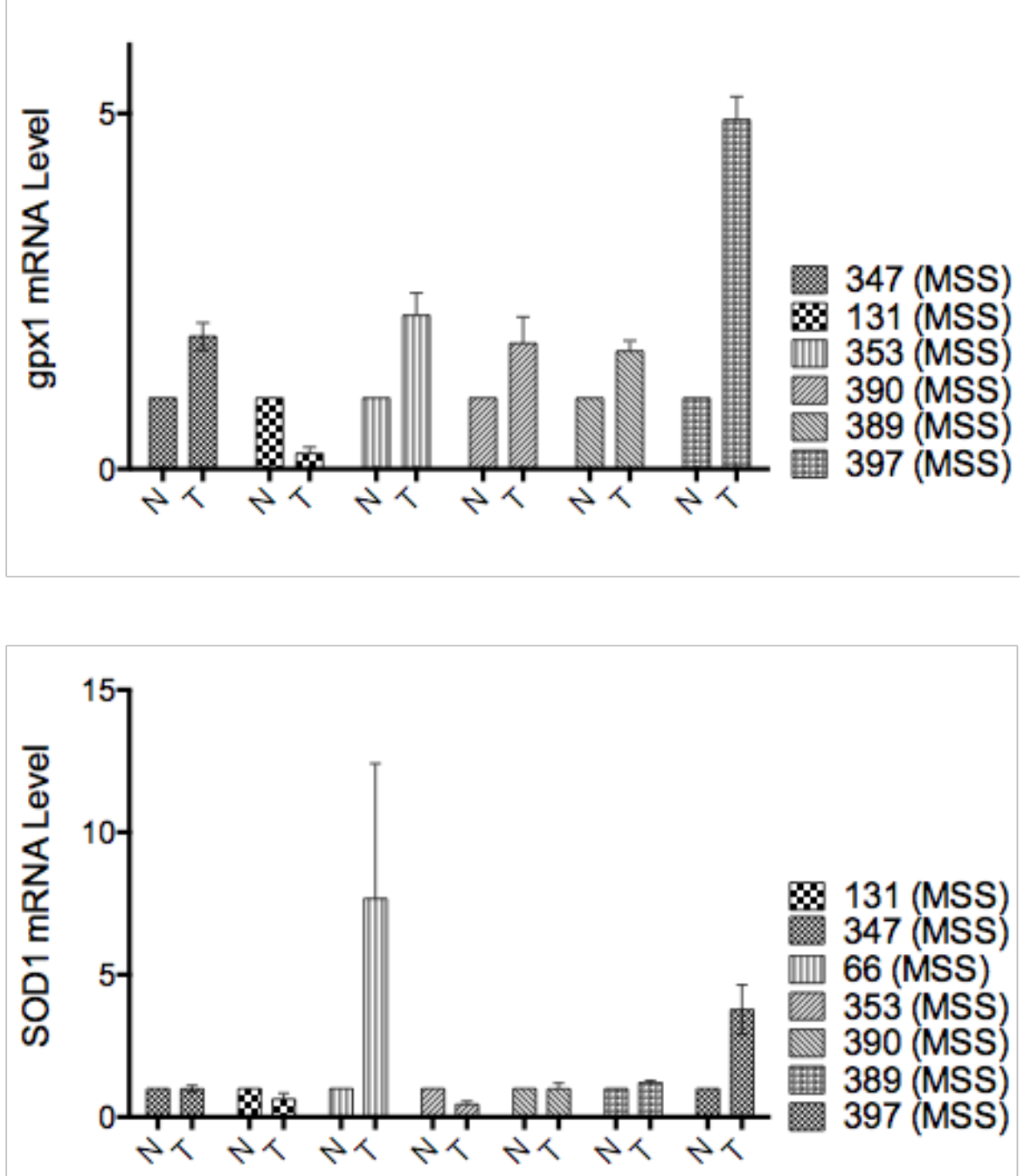
Figure 4.16. MSS tumours do not show a significant increase in expression of the antioxidant genes.

(A-F) qRT-PCR was used to measure the expression of several genes in the antioxidant system in MSS tumours compared to surrounding normal tissue. MSS tumours did not show an increased expression of (A) PGC1B, (B) NRF1, (C) NRF2, (D) catalase, (E) GPX1 and (F) SOD1 compared to surrounding normal tissue. Results shown here are from two independent experiments carried out in triplicate. Error bars represent stand error of the mean.

\section{Figure 4.17}

\begin{tabular}{|c|c|c|}
\hline \multirow{2}{*}{} & \multicolumn{2}{|c|}{ Average fold change } \\
\hline Gene & MSS & MSI \\
\hline PGC1 $\beta$ & 2.07569247 & 2.007241727 \\
\hline NRF1 & 2.11204977 & 2.276021 \\
\hline NRF2 & 4.21932991 & 2.190205071 \\
\hline Catalase & 2.07029845 & 2.040376571 \\
\hline GPx1 & 2.35512133 & 2.098196083 \\
\hline SOD1 & 2.20891752 & 2.246717714 \\
\hline
\end{tabular}

Figure 4.17. There is no significant difference in the average fold change in expression of the antioxidant genes in MSI tumours compared to MSS tumours.

The average fold change in expression of the antioxidant genes compared to surrounding normal tissue is not significantly different in MSS tumours compared to MSI tumours. The values shown here are from seven tumours in the MSS group and at least eleven tumours in the MSI group from two independent experiments carried out in duplicate. 


\subsubsection{Analysis of mitochondrial phenotypes in other MMR-deficient cells Examining}

\subsubsection{Deficiencies in Complex I in MSH2 and MSH6 deficient cell lines}

Taken together my data conclusively demonstrates that MLH1-deficient cell lines have a deregulated mitochondrial phenotype. Therefore, I next investigated whether other MMR-deficiencies display a similar phenotype or whether my observations are specific to deficiencies in MLH1 only. Initially, we analysed Complex I protein expression in the MSH2- and MSH6-deficient and -proficient matched cell lines using the antibody targeting NDUFB8 (Figure 4.18A-C) Protein was isolated from the MSH2-deficient cell line HEC59 and its isogenetically matched cell line HEC59+chr2 and the MSH6 deficient cell lines DLD1 and U251.TR3 and their matched MSH6 proficient cell lines DLD1+chr2 and U251, respectively. Upon western blotting for NDUFB8 expression, we observed a decrease in NDUFB8 expression in the MSH2-deficient HEC59 cells (Figure 4.18A) and in the MSH6 deficient DLD1 cell line (Figure 4.18B), in comparison to their MMR-proficient matched cell lines. However, a slight decrease in NDUFB8 expression was observed in the MSH6-deficient U251.TR3 (Figure 4.18C) cell line in comparison to the U251 cells. The fact that the MSH6 deficient cell line DLD1 displayed a more pronounced decrease in NDUFB8 expression compared to U251.TR3 could be explained by the fact that these matched cell lines are generated by different methods leading to different cell models. U251.TR3 is made by exposure to Temozolomide and therefore acquires secondary mutations whereas the addition of chromosome 2 to DLD1 cells results in the addition of other genes carried on this chromosome which may affect NDUFB8 expression. 
Figure 4.18

A

HEC59 HEC59+chr2

(MSH6-VE) (MSH6+VE)

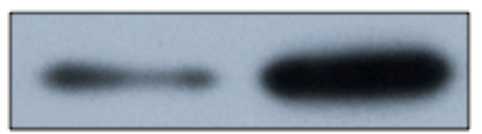

NDUFB8

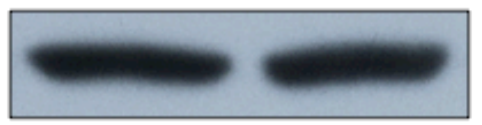

$\beta$-actin

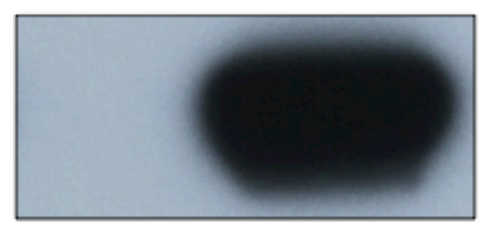

$\mathrm{MSH} 2$

C

1

U251.TR3

U251

(MSH6-VE) (MSH6+VE)

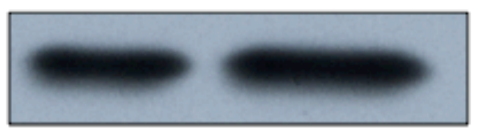

NDUFB8

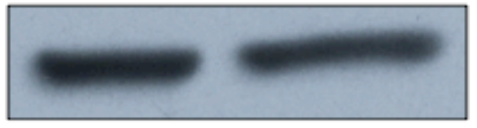

$\beta$-actin

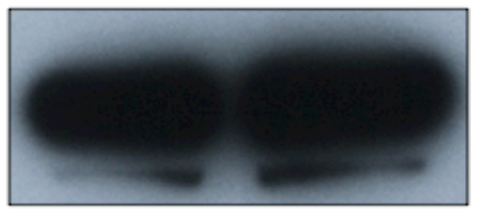

MSH6
B
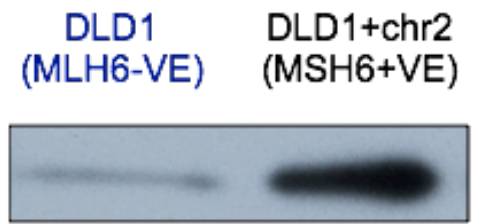

NDUFB8

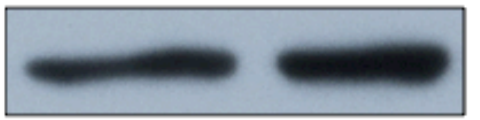

$\beta$-actin

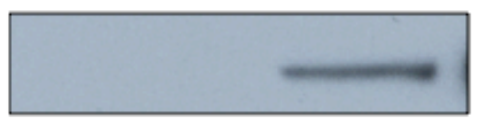

MSH6 
Figure 4.18. MSH2 and MSH6 deficient cell lines have decreased expression of Complex I.

(A-C) Western blot analysis to investigate the expression of Complex I in the absence of MSH2 and MSH6. Protein lysates were isolated from the MSH2 deficient cell lines cell line HEC59, the MSH6 deficient cell lines DLD1 and U251.TR3, the MSH2 proficient cell line HEC59+chr2 and the MSH6 proficient cell lines DLD1+chr2 and U251. These cell lines were analyzed by western blotting using anti-NDUFB8 (accessory subunit of Complex I), MSH2, MSH6 and $\beta$-actin primary antibodies. Quantification was carried out using ImageJ and represents the fold change in protein level (normalized to $\beta$-actin) in relation to the MLH1-deficient cell line HT116. 
To further investigate the decreased Complex I expression in the MSH2 and MSH6 deficient cells, we next measured the activity of Complex I activity, as before. Measuring Complex I activity would allow me to see if there are differences in Complex I as a whole as opposed to a single subunit. Upon carrying out the ELISA to measure Complex I activity, I did indeed see a decrease in activity in all the MMR-deficient cells analysed (Figure 4.19A-4.19C). Given that Complex I activity is likely to be more biologically relevant than examining the expression of the individual Complex I subunits, this result displaying decreased Complex I activity in all my MMR-deficient cell lines lead me to further investigate whether these cell lines displayed deficiencies in mitochondrial function and the antioxidant defense system. 


\section{Figure 4.19}

A

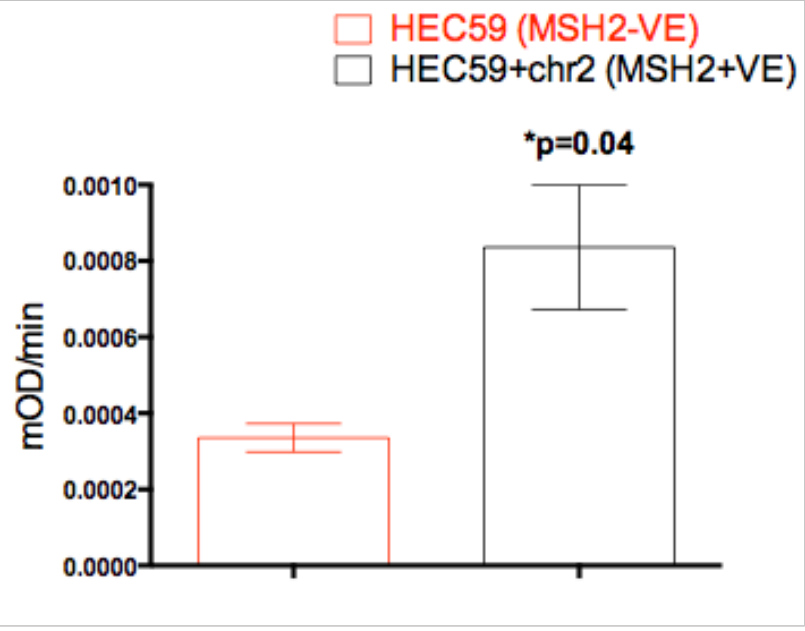

C

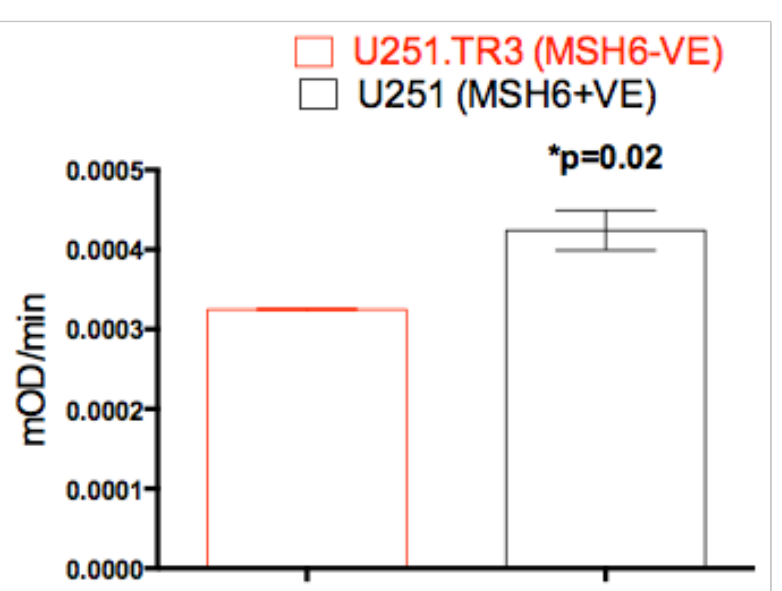

B

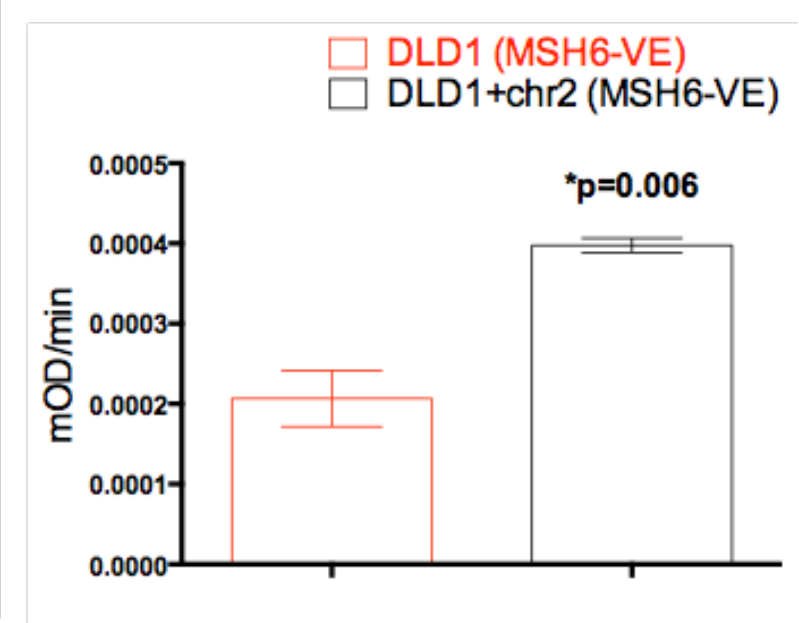

Figure 4.19. Complex I activity is decreased in MSH2 and MSH6 deficient cell lines.

(A-C) An ELISA was used to measure Complex I activity. Protein lysates were isolated and quantified and equal amounts of protein were incubated in the ELISA plate to determine the activity of Complex I by measuring the oxidation of NADH to NAD+ and the simultaneous reduction of a dye leading to increased absorbance at $450 \mathrm{~nm}$, over time. Complex I activity was measured using the ELISA described with protein lysates from the (A) MSH2-deficient HEC59 and MSh2- proficient HEC59+chr2 cell lines. (B) The MSH6-deficient DLD1 and MSH6proficient DLD1+chr2 and (C) The MSH6 deficient cell line. (A-C) The graphs shown here are from three independent experiments carried out in triplicate. 
4.4.2.2 MSH2 deficient cell lines have decreased mitochondrial copy number and decreased expression of the antioxidant defense enzymes.

I wanted to investigate if MMR deficient cell lines have the same mitochondrial phenotype as I had shown in MLH1-deficient cell lines so I carried out similar experiments as previously mentioned to determine expression of $P G C 1 \beta$, antioxidant defense genes and mitochondrial copy number in my panel of MMR deficient and proficient cell lines. Our results suggest that only the MSH2 deficient cell lines HEC59 displayed decreased expression of the antioxidant defense genes (Figure 4.20A-4.20F) and decreased mitochondrial copy number (Figure 4.21A) compared to its matched MSH2 proficient cell lines HEC59+chr2. 
Figure 4.20

A

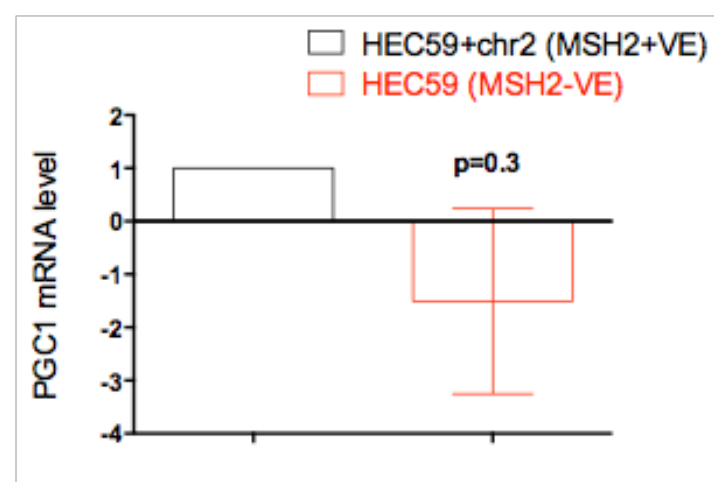

C

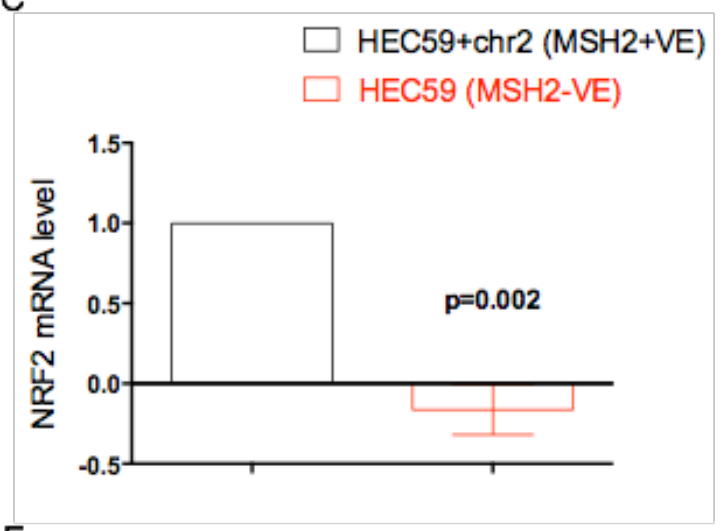

E

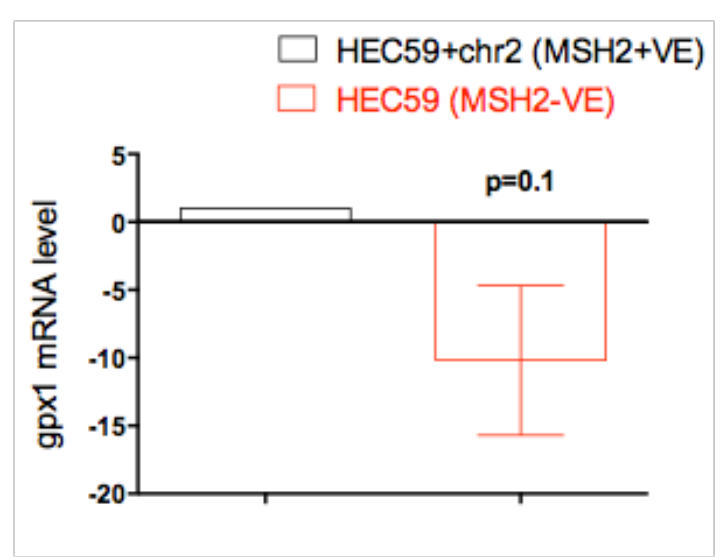

B

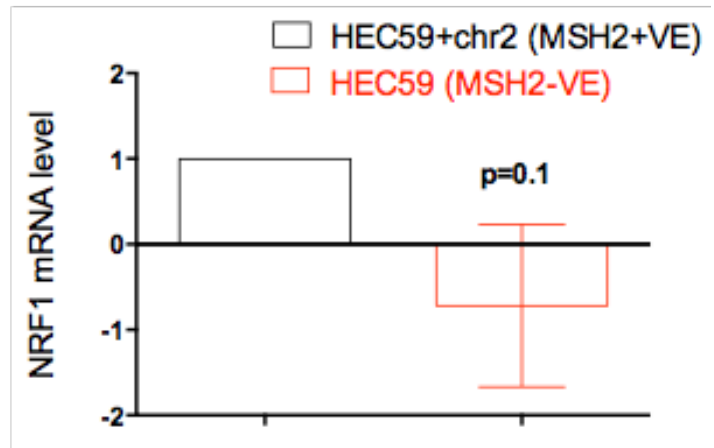

D HEC59+chr2 (MSH2+VE) HEC59 (MSH2-VE)

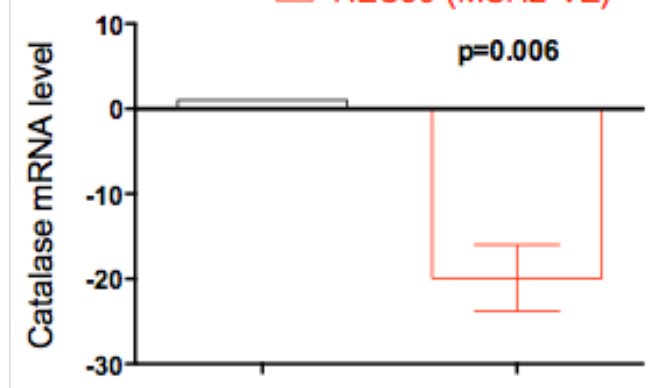

F

HEC59+chr2 (MSH2+VE)

HEC59 (MSH2-VE)

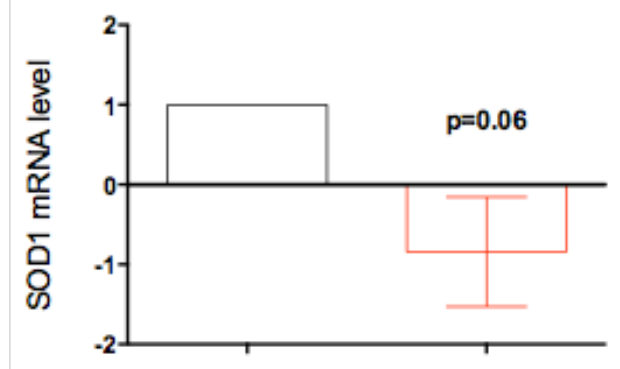

${ }^{*} p<0.1$

${ }^{* *} p<0.05$

${ }^{* \star *} p<0.01$ 
Figure 4.20. The MSH2 deficient cell line HEC59 has decreased expression of the antioxidant defense genes.

(A-F) qRT-PCR was used to measure the expression of several genes in the antioxidant system in the MSH2 deficient cell line HEC59 compared to its isogenetically matched MSH2 proficient cell line HEC59+chr2. Decreased expression of (A) PGC1ß (B) NRF1, (C) NRF2, (D) catalase, (E) GPx1 and (F) SOD1 was observed in the MLH1-deficient cell lines. Results shown here are from three independent experiments carried out in triplicate. Error bars represent standard error of the means. 


\section{Figure 4.21}

A

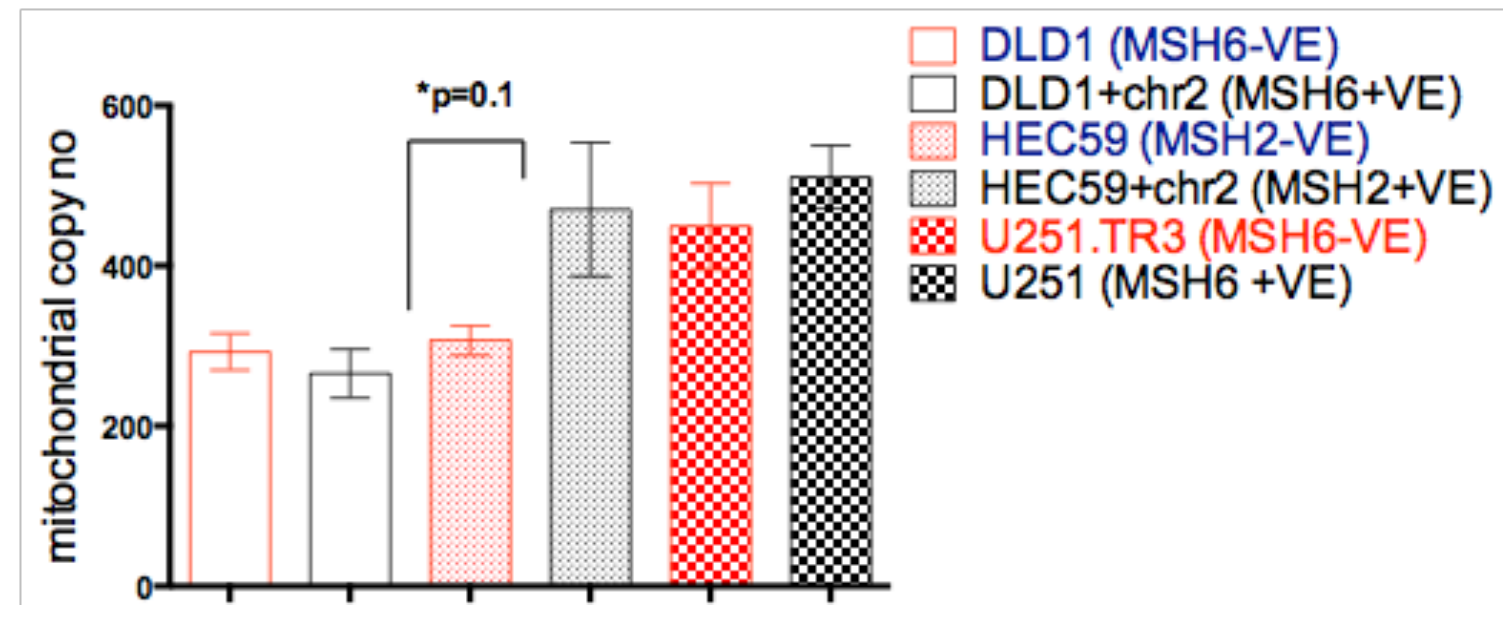

Figure 4.21. The MSH2 deficient cell line HEC59 has decreased mitochondrial copy number.

(A) Mitochondrial copy number was determined in the MSH2 deficient cell line HEC59 and the MSH2 proficient cell line HEC59+chr3 using qPCR with genomic DNA. Similar experiments were carried out in the MSH2 deficient cell lines DLD1 and TR3.U251 as well as their matched MSH6 proficient cell lines DLD1+chr2 and U251. There was a significant decrease in mitochondrial copy number in the MSH2 deficient HEC59 cell line compared to the MSH2 proficient cell line HEC59+chr2. Results of three independent experiments carried out in triplicate are shown here. Error bars represent standard error of the means. 
Given the mitochondrial phenotype I had observed in my MLH1-deficient cell lines I wanted to investigate if a similar phenotype was present in other MMR deficient cell lines. I used western blot analysis to look for the expression of Complex I (NDUFB8 subunit) in a panel of MMR-deficient and proficient isogenetically matched cell lines. I observed a decrease in the expression of NDUFB8 in the MSH2 deficient cell line, HEC59 as well as one of the MSH6 deficient cell lines, DLD1 compared to their respective matched MMR proficient cell lines, HEC59+chr2 and DLD1+chr2, respectively. In a similar fashion to my previous experiments, I carried out an ELISA in these cell lines to examine the activity of Complex I. There was a corresponding decrease in activity of Complex I in all the MMR deficient cell (DLD1, HEC59 and U251.TR3) lines compared to the corresponding MMR proficient cell lines (DLD1+chr2, HEC59+chr2 and U251). Furthermore I observed a similar mitochondrial phenotype with decreased mitochondrial copy number as well as decreased expression of PGC1 $\beta$ and NRF1 and decreased expression of the antioxidant defense enzymes NRF1, NRF2, catalase, GPX1 and SOD1 in the MSH2 deficient cell line HEC59 compared to its isogenetically matched MSH2 proficient cell line HEC59+chr2. 


\section{Discussion}

\subsection{Identification and characterization of novel therapeutics for the treatment of MLH1 deficient cancers}

MMR deficiency is involved in the pathogenesis of a significant proportion of several common cancers and MLH1 loss specifically is one of the most common causes for MMR deficiency in these tumours. Several studies have shown that MMR-deficient tumours are resistant to commonly used chemotherapeutics, so there is an unmet clinical need to identify new agents to treat this group of cancers [85]. Numerous lines of evidence including a preliminary screen that identified mitochondrial drugs to be selectively lethal with MLH1 deficiency, have suggested that targeting the mitochondria in MLH1 deficient disease might be a useful novel strategy [79, 187]. There is little known about the MMR pathway and its role in the mitochondria. With the recent move in clinical practice towards personalized cancer care, using a synthetic lethal approach has proved successful in identifying drugs and targets that affect tumour cells whilst sparing normal body cells $[84,188,376]$. To this end, the overall aim for this $\mathrm{PhD}$ project is to employ a synthetic lethal approach and identify drugs that target mitochondrial function, which are synthetically lethal with MLH1 deficient cancers. This line of investigation will allow me to explore the relationship between the MMR pathway and more specifically MLH1 and the mitochondria.

\subsubsection{Validation of mitochondrial-targeted agents in a range of MLH1-deficient and proficient cell lines.}

To identify drugs that target mitochondrial function that are synthetically lethal with MLH1 deficient cell lines, I tested several drugs identified as hit compounds from a previous drug screen carried out by our lab in MLH1 deficient and proficient cell lines. Several other drugs were identified from a review by Fulda et al [373] on targeting the mitochondria for cancer therapy. Short term cell viability assays were carried out with all these drugs in an isogenetically matched colorectal cell line HCT116 which is MLH1 
deficient and HCT116+chr3 which is MLH1 proficient as well as in a panel of MLH1 deficient and proficient cell lines from a variety of tumour types (Table 4.1).

The isogenic matched paired models used in this study are generated by using a parental cell line harbouring a mutation in one of the MMR genes (HCT116, DLD1, HEC59) and the matched proficient cell line is generated by the stable addition of a chromosome (HCT116+chr3, DLD1+chr2, HEC59+chr2) [129]. An alternative method widely used to establish matched cell lines is to expose the parental cell line which is MMR proficient (U251) to a particular drug for a period of time so that an acquired mutation is established (U251.TR3 made by exposure to TMZ). The resulting MMR deficient cell lines made by this method not only differ in their MMR status but also differ with regards to secondary mutations. The matched cell lines result in cells with an identical genetic background whereas the panel of cell lines I used have diverse genetic backgrounds.

I feel the strategy I employed for testing these drugs is robust since they were all tested in an isogentically matched cell line to confirm that the selectivity is due to MLH1 deficiency and then tested in a panel of isogenetically diverse cell lines to see if my results are generalizable to a range of tumour types.

The obvious advantage of using a matched cell line made by the addition of a chromosome harboring the MMR gene of interest is that it allows more accurate identification of synthetic lethal interactions with MMR genes. Conversely, the criticisms directed towards using this approach is that the chromosome contains numerous other genes aside from the MMR gene of interest. One could argue that matched cell lines generated by an acquired mutation are more clinically relevant since the secondary mutations they possess are thought to drive the oncogenic process .

\subsubsection{The ROS inducing agent Parthenolide is selectively lethal with MLH1- deficient cell lines}

MLH1 deficient cell lines were found to be synthetically lethal with the ROS inducing agent Parthenolide both in the matched cell lines HCT116+/-chr3 (Figure 4.2A) and in a panel of MLH1 deficient and proficient cell lines (Figure 4.3A-4.3C). The selectivity with 
MLH1 deficient cell lines was not apparent with agents that target various other aspects of the mitochondria (Table 4.2) (Figure 4.4A-4.4E).

The fact that Parthenolide was selectively lethal with MLH1 deficiency in both the isogenetically matched cell lines and the panel of cell lines strongly suggests that the reason behind the selectivity is MLH1 deficiency. Furthermore, the fact that only Parthenolide showed selectivity and not the other mitochondrial-targeted agents suggests that the selectivity of Parthenolide is not merely because it is a mitochondrialtargeted agent.

Parthenolide is a sesquiterpene lactone found in the plant Tanacetum parthenium. It is most well known for its anti-inflammatory activity but several studies have recently shown it to be an effective chemotherapeutic through ROS generation via activation of NOX and inhibition of several FOXO3a target antioxidant enzymes including manganese SOD2 and catalase.[377, 378]. A recent study by D'Anneo et al in triple negative breast cancers illustrated that Parthenolide and its soluble analogue dimethylamino (DMAPT) caused cell death by a strong induction of ROS through activation of NOX, depletion of thiol groups and glutathione, activation of JNK and down regulation of nuclear factor NF-kB [379].

\subsection{Investigating the mechanism behind the synthetic lethal relationship between Parthenolide treatment and MLH1 loss}

\subsubsection{Increased ROS production in MLH1-deficient cell lines treated with Parthenolide}

To confirm that the selectivity of Parthenolide with MLH1-deficient cell lines is due to ROS production I carried out experiments using the dye DHE to measure ROS levels in the MLH1-deficient cell line HCT116 and the MLH1-proficent cell line HCT116+chr3 on the the MitoXpress machine. DHE is dye which is widely used to detect superoxide anions by the fact that it becomes oxidized and fluoresces upon reacting with these molecules. Carlisi et al have shown that Parthenolide only produces modest DHE signal 
in the first 5 hours and that this progressively increases to a maximum from 8-16 hours [363]. Upon optimizing this experiment I actually decided to measure ROS levels shortly after Parthenolide was added since there was significant cell death when the cells were left for an extended period of time in Parthenolide which could also affect ROS levels. Carlisi et al used flow cytometry to measure fluorescence but I used a high throughput fluorescent microscope, which may be more sensitive and therefore detect ROS levels earlier. [363]. I observed that the cytosolic ROS levels at baseline (DMSO treated cells) were not significantly different between the MLH1-deficient and proficient cell lines (Figure 4.5B) but increased ROS levels in the MLH1- deficient cell lines became apparent upon treating the cells with the ROS inducing agent Parthenolide (Figure 4.5C). The addition of NAC to the same cell lines treated with Parthenolide was able to rescue the selectivity (Figure 4.5D). These experiments established that ROS was likely to play a significant role in the selectivity of Parthenolide with MLH1 loss.

\subsubsection{MLH1-deficient cell lines do not have a synthetic lethal relationship with other ROS inducing agents}

I did not observe as robust a selective toxicity with MLH1 deficient cell lines using two other ROS-inducing agents; Menadione and $\beta$-lapachone (Figure 4.6A-4.6B) compared to Parthenolide. This may be due to the different methods in which these drugs induce ROS.

Menadione (Vitamin K3) is a quinone that is metabolized by the reductive enzymes NADPH-cytochrome p450 and NADH-ubiquinone oxidoreductase by one electron reduction. This process initiates futile redox cycling when the resulting semiquinones enter the electron transport chain causing ROS generation [365, 366]. Many pre-clinical studies have shown Menadione, through its ability to generate ROS to be a potential treatment for cancers. The study by Yang et al found CR108 (a novel vitamin K3 derivative) to cause cell death in human breast cancer cells by causing ROS production, apoptosis and mitochondrial dysfunction [367]. $\beta$-lapachone (clinical form, ARQ761), induces ROS by also undergoing futile redox cycles on the respiratory chain but in an NQ01 dependent manner [368]. 


\subsection{Functional Investigation to study the differences between mitochondrial function in MLH1-deficient and proficient cell lines}

\subsubsection{Investigating the antioxidant response in MLH1 deficient and proficient cell lines}

I decided to investigate the antioxidant defense system in my cell lines given the vital role of this system in the control of ROS levels and the fact that this system is one of the targets of Parthenolide. I observed a significant decrease in both the gene expression (NRF2, SOD1, Catalase and GPX1) (Figure 4.7A-4.7D) and protein levels (NRF2, catalase and GPx1) (Figure 4.8A-4.8C) of several key members of the antioxidant defense system in the MLH1-deficient cell line HCT116 compared to its isogentically matched pair HCT116+chr3.

The transcription factor NRF2 (NFE2L2) through its interactions with the antioxidant response element (ARE) is probably the most well established regulator of several enzymes within the antioxidant defense system [113]. Some studies have shown NRF1 to have a role independent of NRF2 in activating ARE-driven genes but its part in controlling the antioxidant system is less well defined [369]. Unlike the other ROS agents used in this study, Parthenolide has been shown to inhibit members of the antioxidant defense system which is likely to explain why only Parthenolide showed selectivity with MLH1-deficient cell lines which already have an attenuated antioxidant response. Furthermore a recent study by Hassane et al supports our finding since they identified NRF2 through a gene signature analysis to be upregulated as a protective mechanism when primary human acute myeloid leukemia (AML) cells are treated with Parthenolide [370]. With this Parthenolide gene expression signature they carried out chemical genomic screening of the Connectivity Map database and found that compounds acting along the phosphatidylinositol 3-kinase and mammalian target (PI3K) and mammalian target of rapamycin pathway (mTOR) inhibited this cytoprotective responses. The authors used the drug Temsirolimus for their experiments which is a known mTOR inhibitor and they were able to show that Temsirolimus inhibits NRF2 and its downstream activation of the antioxidant enzymes. They carried out in vitro experiments treating AML cells with Parthenolide and Temsirolimus versus Parthenolide 
alone and in vivo experiments in murine xeno transplantation models using DMAPT (water soluble analogue of Parthenolide) with Temsirolimus or DMAPT as a single agent. Compared with Parthenolide alone, the combination treatment was synergistic and significantly decreased viability of AML cells and decreased tumour growth in vitro [370]. From this study we gain further evidence that cell lines deficient in NRF2 and the antioxidant defense enzymes, as is the case with my MLH1 deficient cell lines, are more sensitive to the ROS inducing agent Parthenolide due to its mechanism of action.

As mentioned previously, the ROS levels at baseline (DMSO treated cells) between the MLH1-deficient HCT116 cell line and the MLH1-proficient cell line HCT116+chr3 were not significantly different and the difference only became apparent upon ROS induction. It has been established that ROS levels are tightly controlled since they play an essential role in cell signaling and maintaining cell function. The electron transport chain is known to be one of the main sites of ROS production and deficiencies of the complexes involved in this system are known to alter ROS levels. The redox status of the electron transport chain is one of the main determinants of ROS production and typically deficiencies in the chain are likely to increase ROS levels. It is thought that baseline ROS levels are maintained to allow cellular functions but following the introduction of stress these levels may change [371]. In this study the baseline ROS levels are similar in the MLH1-deficient and proficient cell lines despite an attenuated antioxidant defense system, this finding is likely to be due to the tight control of baseline ROS levels to allow normal homeostasis.

\subsubsection{The antioxidant response in cancer}

The expression of antioxidant enzymes have been found to be altered in many cancers but the evidence thus far does not seem to point to a specific pattern in which the antioxidant enzymes are altered in all cancers. Several small studies have been carried out examining the difference in expression between cancer tissue and surrounding normal tissue. Botwick et al examined the expression of SOD1, SOD2, catalase and 8hydroxydeoxyguanosine as a marker of oxidative stress in human prostate carcinoma tissue, high grade prostatic intraepilethial neoplasia (PIN) and benign epithelium [410]. The authors found decreased expression of all the antioxidant enzymes in PIN and the prostate cancer samples compared to the normal tissue. There was no difference in the 
levels of the DNA adduct 8-hydroxydeoxyguanosine in any of the different types of samples, eluding to the fact that oxidative stress occurs early in the development of cancer [410]. Chung-man Ho et al looked at the expression of the antioxidant enzymes in 16 lung tumour samples (adenocarcinoma and squamous cell) and 24 tumour free samples [411]. The authors examined both protein and mRNA expression and found decreased total levels of the SODs, decreased catalase expression and similar levels of glutathione and glutathione peroxidase in tumours compared with tumour-free lung tissues [411]. Studies have also shown that the environmental context can affect antioxidant expression as shown in the study conducted by Skrzycki et al which showed decreased levels of total SOD in CRC cell lines (SW480 and SW620) in hypoxia compared to atmospheric normoxia [412]. Recently a study by Govatati et al examined the mitochondrial $D$ loop region of $35 \mathrm{CRC}$ patient samples compared to normal tissue examining for the incidence of mitochondrial $\mathrm{MSI}$ (mtMSI) in this region and then correlated the findings to SOD2 expression [413]. The authors concluded that there was a significant increase in the incidence of mtMSI in tumour samples and that this had a positive correlation with SOD2 overexpression [413].

I possibly would have expected to observe similar results to the study by Govatati et al displaying mtMSI and upregulation of antioxidant enzymes in my MMR deficient cell lines. I did not observe an increased incidence of mtMSI in the D-loop region of these cells compared to the MMR proficient cell lines. However, one potential reason for this is that I was comparing MMR proficient and deficient CRC cell lines and not normal tissue compared to CRC tissue. To ultimately determine whether MLH1 deficient tumours have increased mtMSI compared to normal tissue, an extensive in-depth analysis of fresh tissue is required. Furthermore, the main obstacle with investigating mitochondrial mutations is related to the consequences of mitochondrial heteroplasmy where there are multiple mitochondrial genotypes within one cell making it difficult to detect low level mutations. The use of more high depth sequencing may also help determine the precise differences in mtMSI

\subsubsection{Investigating Complex I in MLH1 deficiency}

To investigate if there are functional differences in mitochondrial function between MLH1 deficient and proficient cell lines I examined the expression of the five membrane 
bound oxidative phosphorylation complexes. I established that the MLH1 deficient cell line HCT116 has decreased protein expression of Complex I (Figure 4.9A-4.9B) (Complex I subunit NDUFB8) and decreased gene expression of both mitochondrial (Mt-ND5, Mt-ND2) (Figure 4.10A-4.10B) and nuclear encoded (NDUFA) (Figure 4.10C) Complex I subunits compared to its isogentically matched cell line HCT116+chr3. Furthermore I observed a significant decrease in the activity of Complex I in the MLH1 deficient cell line HCT116 compared to its isogenetically matched MLH1-proficient cell line HCT116+chr3 (Figure 4.12A). There was also a similar decrease in Complex I activity in a panel of MLH1 deficient cell lines compared to a panel of MLH1 proficient cell lines (Figure 4.12B).

As described above, Mendione and $\beta$-Lapachone produce ROS through a similar mechanism which is different to the mechanism of action of Parthenolide. After examining the differences in mitochondrial function between my MLH1 deficient and proficient cell lines it became apparent that the reason why only Parthenolide is selectively lethal with MLH1 deficient cell lines may be due to its mechanism of action involving the antioxidant defense system in MLH1 deficient cells which already posses deficiencies in Complex I and the antioxidant defense system.

I did not observe a difference in the expression of NDUFB8 between a panel of MLH1proficient and deficient cell lines (Figure 4.11A) which could potentially have explained the observed selectivity of this panel of MLH1-deficient cell lines with Parthenolide. Given that Complex I is the biggest complex in the oxidative phosphorylation chain and composed of thirty seven subunits it is not surprising that I did not see a difference in expression of one of the subunits in a genetically diverse panel of cells. When I examined complex I activity which is more biologically relevant, a significant difference in Complex I expression became apparent between the MLH1-deficient and -proficient cell lines in my panel of cells (Figure 4.12B).

\subsubsection{Complex I and MMR deficient cancer cell lines}

The first potential link between Complex I and the MMR system is that of Habano et al who examined 45 sporadic colorectal samples and identified mutations within microsatellites mainly within the non-coding $D$-loop, however $7 \%$ had mutations within 
the microsatellites in the Complex I genes ND1 and ND5 [247]. More recently, Mori et al examined coding region frameshift mutations within $46 \mathrm{MSI}-\mathrm{H}$ tumours and identified that in $28 \%$ of these tumours, there was a mutation in the nuclear encoded B14.5B Complex I subunit [380].

Complex I deficiencies have clearly been demonstrated in a multitude of studies and the concept of deficiencies in this complex in the context of cancer is now widely accepted. Furthermore there is already some evidence linking complex I deficiencies with MMR deficient cell lines and this study adds further strength to this area of research

\subsubsection{MLH1 deficient cell lines have decreased basal OCR and spare respiratory capacity}

I used the XF analyzer to see whether the deficiencies I had observed in Complex I expression resulted in changes in the basal OCR and SRC. The basal OCR gives a measure of basal oxidative phosphorylation whilst the spare respiratory capacity allows a measure of the ability of the cells to respond to stress and the resulting increased energy demands[88]. Birsoy et al harecently shown that both defects in glucose utilization and oxidative phosphorylation have an impact on spare respiratory capacity. Particularly relevant to my study, the authors demonstrated that cell lines with known mutations in Complex I subunits (but no defects in glucose transporters) were unable to sufficiently increase their OCR to meet increased demands when grown in low glucose media. To further prove that the defect in SRC was due to Complex I the investigators demonstrated an increase in the OCR (and SRC) in low glucose conditions in these cell lines when the Complex I gene NDII from s. cerevisiae was expressed [372].

I observed a significant decrease in both the OCR and SRC in my MLH1- deficient cell line HCT116 compared to the MLH1-proficient cell line HCT116+chr3 (Figure 4.13A4.13C). On carrying out similar experiments in my panel of cell lines there was a decrease in the basal OCR in my panel of genetically diverse MLH1-deficient cell lines compared to the MLH1-proficient cell lines (4.13D). These results suggest that MLH1deficient cell lines have decreased oxidative phosphorylation and have a decreased capacity to respond to increased energy demands and this is likely due to the deficiencies in Complex I which I observed. 


\subsubsection{Respiratory chain complex deficiencies in cancer}

Within the context of cancer, mutations in the mitochondrial encoded respiratory complex genes has been shown in various tumour types affecting mainly $\mathrm{Cl}, \mathrm{CIII}$ and CIV [505]. It is still unclear what the definitive course of events is within these tumours; the main question is whether these mutations cause cancer or arise due to the high levels of oxidative stress in the tumour compounded by the fact that mtDNA replication and repair occurs with poor fidelity. It is also paramount to remember that any definitive conclusions about mtDNA are affected by the fact that there are multiple copies of the mitochondrial genome within the numerous mitochondria in each cell. This could lead to heteroplasmy where there are multiple mitochondrial genotypes within one cell or homoplasmy where all the DNA within all the mitochondria in one cell are the same [505]. There is evidence that some mitochondrial mutations even when present in a homplasmic state may be merely due to unbiased mtDNA replication and cell division alone [506]. Ishikawa et al however carried out a very important study in 2008, which showed that when mtDNA in a non- metastatic mouse tumour was replaced by mtDNA from a metastatic tumour, there was a significant transformation in the metastatic potential of the non- metastatic cell lines [507]. Furthermore, when the reverse experiment was carried out the metastatic potential of the aggressive cell lines was blunted [507]. Several studies have been carried out with the aim of investigating the consequences that mitochondrial respiratory complex mutations, especially Complex I, have on tumours. Park et al [5] developed rotenone resistant clones harbouring homoplasmic and heteroplasmic mtDNA mutations with a resulting abnormality in oxidative phosphorylation. They developed C8T and C9T cells with a frame-shift mutation in the ND5 gene which was present in nearly all mtDNA (72\% mutant in C8T and near homoplasmic DNA in C9T). The mutation that was found in ND5 by this method had previously been found by Polyak et al. [508] in human colorectal cancers. Cybrids were then created by transferring the mitochondria from the C8T and C9T cells to the human osteosarcoma cell line 143B. The authors found that increasing mutant ND5 was associated with decreased oxidative phosphorylation, increased ROS, increased glycolysis and tumour growth. Bonora et al. [509] and Ishikawa et al. [507] have also shown that mutations in mitochondrial encoded Complex I subunits, ND1 and ND6 respectively, resulted in a decrease in Complex I activity and increased ROS production. Interestingly, Yao et al. [510] have recently shown that rendering the non 
small cell lung cancer cell line A549 resistant to Cisplatin by exposing them to low concentrations of the drug for a prolonged period of time, resulted in the transformation of a mutation in the MT-ND2 gene which was present in a heteroplasmic state in the control cells into a homoplasmic mutation in all the Cisplatin resistant cells. These cells harbouring a mutation in one of the core subunits of Complex I resulted in a $50 \%$ decrease in Complex I activity with maintained oxidative phosphorylation and increased expression of some of the nuclear encoded subunits of Cl-CIV and of mtDNA encoded COXI (subunit of CIV). The authors investigated how these cells were able compensate for the decrease in Complex I activity and implicated the nuclear co-activators PGC-1a and PGC-1 $\beta$ evidenced by an increase in their mRNA levels[510].

\subsubsection{Investigating differences in the mitochondrial genome between MLH1- proficient and deficient cell lines}

I carried out sequencing of the mitochondrial genome to investigate if the deficiencies in Complex I that I observed may be due to $\mathrm{MSI}$ within Complex I genes in the mitochondria. A study by Habano et al discovered that out of 45 sporadic colorectal cancers, $44 \%$ had mutations within microsatellites in the non-coding D-loop region of the mtDNA and tumours also had mutations within mitochondrial encoded Complex I genes [247]. This study was the first indication that MMR deficient tumours may harbor mutations within Complex I genes. More recently, Mishra et al proved that MLH1 deficiency (as a result of retinal endothelial cells exposed to high glucose) leads to increased sequence variants in the mitochondrial D-loop region and decreased OCR. All of these finding were ameliorated when MLH1 was overexpressed [249]. I did not observe any differences in mutation rates between MLH1-deficient and proficient cell lines but it is important to discuss a few experimental considerations that may have led to this result.

One of the challenges of sequencing the mitochondrial genome and diagnosing mitochondrial disease is the fact that the mitochondria within each cell could be heteroplasmic where there are multiple genotypes within one cell or homoplasmic where all the mtDNA within one cell is the same. To complicate the scenario further, heteroplasmy is not a static situation and mutational load can change during mitotic and meiotic division. In the case of mitochondrial disease the resulting phenotype or 
biochemical change depends on the amount of mutant DNA present and the threshold of the affected tissue before symptoms or a phenotype become apparent. It is widely understood from numerous studies that mitochondrial DNA is constantly acquiring mutations and that homoplasmy in the true sense is unlikely to exist but the most likely situation is that these mutations are present at a low level and cannot be detected [375, 376]. My experiment allowed for a depth of $18,000 \mathrm{bp}$ which is the average number of reads that cover known reference bases and this value gives an indication of the degree of certainty with which a variant is detected.

King et al carried out NGS on the Illumina MiSeq ${ }^{\text {TM }}$ platform using DNA from 283 blood samples and found that one of the problems they encountered is that the coverage was not equal across the mitochondrial genome within individual genomes especially in a portion of the ND1 gene (Complex I) [377]. This type of error may have affected my results. Furthermore, it is difficult to know at what point after the addition of chromosome three bearing MLH1 $(\mathrm{HCT} 116+\mathrm{chr} 3)$ do the mutations that were present in the MLH1-deficient parental cell line (HCT116) become repaired. The investigators who constructed this matched cell line carried out experiments approximately fourteen days after chromosome three was transferred to the MLH1-deficient parent cell line HCT116 examining whether a mutation within a specific microsatellite had been repaired. They reported that after the addition of chromosome three, 8 of 80 HCT116 (MLH1- deficient) sub-clones carried the mutation in comparison to none of the 225 HCT116+chr3 (MLH1proficient) sub-clones confirming restoration of MMR activity. Furthermore, this was accompanied by changes in response to drug treatment [93] Although I did not observe any significant differences in mutations within mitochondrial encoded Complex I genes, it is worth bearing in mind that I did observe a decreased expression of several mitochondrial encoded Complex I subunits in my MLH1-deficient cell lines. These finding suggest that there are likely to be mutations within Complex I genes in the MLH1-deficient cell lines but these may be low levels of heteroplasmic mutations we did not observe due to experimental constraints or possibly due to alterations in translational and post translational modifications. An alternative explanation is that there may be increased mutations in the MLH1-deficient cell lines in nuclear genes essential for mitochondrial DNA maintenance resulting in Complex I deficiencies. 


\subsubsection{Investigating mitochondrial biogenesis in MLH1-deficient cell lines}

Experiments investigating mitochondrial biogenesis in the MLH1-deficient cell line HCT116 and the MLH1-proficient cell line HCT116+chr3 revealed decreased expression of the nuclear transcriptional co-activator PGC-1 $\beta$ (Figure 4.14A) and its downstream transcription factor NRF1 (Figure 4.14B-4.14C) as well as decreased mitochondrial copy number in the MLH1-deficient HCT116 compared to MLH1-proficient HCT116+chr3 cell lines (Figure 4.14B).

We measured mitochondrial copy number using $\mathrm{PPCR}$ to examine the expression of the mitochondrial $t R N a$ relative to the expression of the nuclear housekeeping gene $\beta 2 M$. Since mitochondrial DNA codes for the respiratory genes that are essential for the cell's energy processes; the replication, transcription and expression of these genes are known to be strictly regulated to match the energy requirements of the cell [380]. Mitochondrial copy number is not only a product of mtDNA itself but also the numerous nuclear proteins involved in control of mtDNA..

The PGC-1 family of co-activators are often described as the master co-regulators of mitochondrial biogenesis since they have diverse roles in controlling mitochondrial biogenesis and function. The mitochondrial phenotype which I have established is present in MLH1-deficient cell lines could be explained by the decreased expression of $P G C 1-\beta$ seen in these cell lines. The key processes involved in mitochondrial biogenesis include transport of nuclear encoded mitochondrial proteins, translation of mitochondrial encoded respiratory chain proteins and replication of mtDNA. PGC1- $\beta$ through its interactions with a multitude of transcription factors including NRF1 and NRF-2alpha controls the expression of the respiratory chain and the supporting machinery which is needed for the expression of a wide range of mitochondrial and nuclear encoded genes with key functions in the mitochondria [325]. Several in vitro and in vivo studies have shown that overexpression of members of the PGC-1 family including PGC1- $\beta$ results in increased mitochondrial biogenesis [310]. In this study I have demonstrated decreased expression of not only PGC1 $\beta$ but also NRF1 which are involved in Complex I expression and function, oxidative phosphorylation (OCR and spare respiratory capacity) and mitochondrial biogenesis and therefore could explain the phenotype I observe in MLH1-deficient cell lines. Furthermore, PGC1- $\beta$ has also 
been shown to have a role in controlling the antioxidant defense enzymes and therefore the decreased expression of PGC1- $\beta$ that I observed in my MLH1-deficient cell lines is also in keeping with the deficiencies in the antioxidant enzymes in these cell lines. The mechanism behind decreased PGC1 $\beta$ levels in MLH1-deficient cell lines needs to be explored further but given the key role of MLH1 in post replicative repair it is possible that this could be due to mutations in the PGC1- $\beta$ gene.

Several studies have established that MLH1 is likely to have a role in the repair of oxidative DNA damage both in the nucleus and the mitochondria. Colussi et al showed that the baseline levels of the oxidative lesion 8-oxoG was higher in MSH2 and MLH1deficient cell lines [287]. We have previously shown that silencing of the mitochondrial DNA polymerase POLG is synthetically lethal with MLH1-deficient cell lines due to an increase in oxidative DNA lesions in the mitochondria only and not the nucleus [79]. Silencing of several other nuclear encoded mitochondrial genes PINKI, CKMT2 and PCK2 was synthetically lethal with several MMR genes including MLH1 due to an increase in ROS levels and both nuclear and mitochondrial oxidative DNA lesions [187]. In this study I have shown that MLH1-deficient cell lines are selectively lethal with the ROS inducing agent Parthenolide due to the fact that they have deregulated mitochondria, decreased expression of $P G C 1 \beta, N R F 1$ and an attenuated antioxidant defence system. It is important to remember that I found only Parthenolide to be selectively lethal with MLH1-deficient cell lines and not all ROS inducing agents most likely due to the mechanism in which Parthenolide induces ROS. Treatment with Parthenolide results in higher ROS levels in the MLH1-deficient cell lines, which is likely to result in oxidative DNA damage. It is likely that the ultimate reason for decreased cell viability in MLH1-deficient cell lines is due to their inability to repair the oxidative DNA damage in the mitochondria which has occurred due to increased ROS levels compared to MLH1-proficient cell lines. The fact that the MLH1-deficient cell lines have decreased mitochondrial copy number at baseline without the addition of ROS gives a potential insight into the fact that the mitochondrial DNA in these cell lines may already be compromised due to lack of MMR activity. Studies mentioned above support the fact that with increased ROS levels and increased oxidative DNA damage (caused by Parthenolide), MLH1-deficient cell are less able to repair the resulting mitochondrial oxidative DNA damage resulting in decreased cell survival in these cell lines compared to MLH1-proficient cell lines. 


\subsubsection{The significance of mitochondrial copy number changes in cancer}

Based on the fact that there are numerous copies of the mitochondrial genome within each cell and that mitochondria undergo constant fusion and fission the most established way of estimating the number of mitochondrial genomes is to measure mitochondrial copy number per cell. Mitochondrial copy number alterations have been observed in numerous human cancers with both increased [416-419] and decreased copy number alterations [420-422]. Chen et al carried out a large study examining mtDNA copy number in a Chinese cohort of patients and observed an increase in copy number when compared to matched surrounding normal tissue [419]. Van Osch et al compared mtDNA copy number between normal tissue, adenoma and carcinoma tissue and observed a significantly lower copy number in both the cancer tissue and surrounding normal tissue compared to the earlier resected adenoma tissue.

Furthermore, decreased mtDNA copy number was established to be present in the MSI tumours compared to MSS tumours [423].

There is an increased interest in investigating the clinical implications of these copy number changes. Resnik et al studied mitochondrial copy number in cancers by looking at 22 different types of tumours and examining the impact of mitochondrial copy number on expression of metabolic genes [424]. They established that nearly half of the tumours exhibited a reduced copy number compared to adjacent normal tissue. To validate that mitochondrial copy number was a true reflection of mtDNA content the investigators correlated mtDNA copy number with specific gene sets and found that mitochondrial metabolic pathways and the transcription factor TFAM (essential for transcription and replication of mtDNA) were positively correlated to mtDNA copy number across all tumour types. . The investigators explored this further and found that the 'TCA Cycle and Respiratory Electron Transport' gene set was the most frequently correlated to mtDNA copy number (1st out of 674 gene sets) and the other highly scoring sets were mitochondrial metabolism related including mitochondrial beta oxidation of fatty acids and branched chain amino acid catabolism [424]. Gene sets with functions related to mRNA processing, the cell cycle and immune pathways showed a negative correlation with mtDNA copy number. Although in the majority of cancer cases Resnick et al found a positive correlation with mitochondrial genes and mtDNA copy 
number, there were a few tumour types such as bladder cancer samples where this was not apparent [50].

\subsubsection{Some cancers upregulate respiratory genes to compensate for decreased}

\section{mitochondrial copy number}

The concept that in some tumours depletion of mtDNA can actually lead to a compensatory increase in respiratory activity has previously been described. For example, Siedel-Rogol et al demonstrated that when partially mtDNA- depleted cells are cultured there is an upregulation in transcription and replication of mtDNA [425]. Kim et al investigated lipoatrophy due to HIV treatment which is thought to be due to mtDNA depletion. The investigators used samples from the subcutaneous fat of patients with lipoatrophy and compared the DNA, RNA and protein levels from age and body mass index matched controls [426]. The investigators established that although mtDNA copy number was reduced in the lipoatrophy samples, there was evidence of compensation as determined by normal activity of the respiratory enzyme cytochrome $c$ oxidase and MT-CO2 protein levels. There was also evidence of increased mitochondrial biogenesis since several nuclear encoded mitochondrial genes (COX4I1 and UCP2) and pathways were upregulated but interestingly the more conventional mitochondrial biogenesis related transcription factors such as the PGC-1 family, TFAM and NRF1 were at normal or decreased levels [426]. When Resnick et al investigated protein expression (by IHC) in relation to mtDNA copy number in different types of renal cancer tissue (papillary and clear cell) and bladder carcinoma, they found that in $2 / 3$ of tumour types, most of the tumour samples showed decreased mitochondrial protein compared to normal tissue but in the bladder cancer tissue most of the samples exhibited increased mitochondrial protein levels. Interestingly, the bladder cancer samples also did not show a strong correlation between mtDNA copy number and metabolic gene sets but showed a strong correlation with cell cycle and immune response gene sets. Furthermore, the investigators also showed evidence that certain patient samples with specific mutations or copy number alterations correlated with different mtDNA levels. One example of this is that the serous-like endometrial subtype of endometrial cancer which is well known to be characterised by a high number of copy number alterations is positively correlated with increased mtDNA copy number [424]. Overall, the literature seems to point to a correlation between mtDNA copy number and 
respiratory and metabolic gene expression however there is clearly a subset of tumours for which the opposite is true. Further investigation is needed to establish which tumours upregulate mitochondrial function in response to diminished mtDNA copy number and the reason behind this.

The MLH1 deficient cell lines in my study display decreased mitochondrial copy number which has been previously demonstrated by Van Osch et al. Most studies examining the functional relevance of decreased mitochondrial copy number have observed a corresponding decrease in the expression of respiratory chain genes. In my study I established that MLH1 deficient cancers not only have a decreased mitochondrial copy number but also decreased expression of several mitochondrial encoded Complex I subunits (mt-ND2 and mt-ND5 as well as an overall decrease in Complex I activity.

In this study I have shown that MLH1-deficient cell lines are selectively lethal with the ROS inducing agent Parthenolide due to the fact that they have deregulated mitochondria, decreased expression of $P G C 1 \beta, N R F 1$ and an attenuated antioxidant defence system. It is important to remember that I found only Parthenolide to be selectively lethal with MLH1-deficient cell lines and not all ROS inducing agents most likely due to the mechanism in which Parthenolide induces ROS. Treatment with Parthenolide results in higher ROS levels in the MLH1-deficient cell lines, which is likely to result in oxidative DNA damage. It is likely that the ultimate reason for decreased cell viability in MLH1- deficient cell lines is due to their inability to repair the oxidative DNA damage in the mitochondria which has occurred due to increased ROS levels compared to MLH1-proficient cell lines. The fact that the MLH1-deficient cell lines have decreased mitochondrial copy number at baseline without the addition of ROS gives a potential insight into the fact that the mitochondrial DNA in these cell lines may already be compromised due to lack of MMR activity. Studies mentioned above support the fact that with increased ROS levels and increased oxidative DNA damage (caused by Parthenolide), MLH1-deficient cells are less able to repair the resulting mitochondrial oxidative DNA damage resulting in decreased cell survival in these cell lines compared to MLH1-proficient cell lines. 


\subsection{Validating experimental results in patient samples}

To ascertain whether the mitochondrial phenotype that I observed in my MLH1 deficient cell lines is present in tumour samples from patients, I obtained RNA from tumour samples as well as the surrounding matched normal tissue from MSI and MSS samples from a collaborators laboratory (Professor Andrew Silver, Blizzard Institute, QMUL). The MMR defect in MMR deficient tumours is present in the tumour and not the surrounding normal tissue so in theory by comparing these samples any differences due to MMR deficiency should become apparent.

Upon comparing the expression of genes involved in mitochondrial biogenesis PGC1 $\beta$ and NRF1) the antioxidant defense genes (NRF2, SOD1, Catalase and GPX1) in the patient samples, I did not observe a consistent difference in expression of these genes between the tumour samples and matched normal tissue in the MLH1-deficient tumours (Figure 4.15A-4.15F). There was also no significant difference in the expression of these genes between the MSI (Figure 4/15A-4.15F) and MSS (Figure 4.16A-4.16F) tumours (Table 4.17). One of the biggest problems with this sort of analysis is associated with problems due to RNA integrity and separating different types of tissue accurately. The routine use of laser capture microdissection has improved the precision in which tissue samples can be dissected however there are obviously still problems with contamination and with my study it is possible that there was some crossover between tumour tissue and surrounding normal tissue which would obviously affect my results.

Laser capture microdissection does not obliterate problems with RNA integrity and in the study by Vandewoestyne et al describing the advantages and problems associated with two types of laser capture microdissection they carry out a qRT-PCR based RNA amplicon length testing looking for significant differences in ACTB expression between samples [379]. The authors concluded that they observed significant differences in amplicon length between samples and that these differences are due to RNA degradation which is inherent to the LCM system. The authors recommend designing your study to take these problems into consideration like avoiding the use of oligo-dT primers since these do not work well on degraded RNA [379]. It is very likely that some of the considerations mentioned above applied to my experiment and affected the 
ultimate results. Aside from the problems with experimental procedure described above I feel it is important to consider that ROS metabolism is influenced by the environment including factors like hypoxia [371]. The majority of these tissue samples are several years old and it is impossible to know and account for differences in tissue handling and storage which may influence the expression of the antioxidant defense enzymes.

\subsection{Analysis of mitochondrial phenotypes in other MMR-deficient cells}

The differences in the mitochondrial phenotypes I observed within tumours deficient in different MMR proteins compared to their proficient counterparts needs further exploration. All the MMR deficient tumours (DLD1, HEC59 and U251.TR3) displayed decreased expression and activity of Complex I (Figure 4.18A-4.18C and Figure 4.19A4.19C) compared to the corresponding MLH1-proficient cell lines (DLD1+chr2, HEC59+chr2 and U251). Only the MSH2 deficient cell line HEC59 exhibited the complete mitochondrial phenotype I observed in my MLH1 deficient cell lines with decreased expression of members of the antioxidant defense system (NRF2, catalase, GPx1and SOD1) (Figure 4.20A-4.20F) and mitochondrial biogenesis (PGC1 $\beta$, NRF1 and mitochondrial copy number) (Figure 4.21) compared to its isogenetically matched MSH2-proficient cell line HEC59+chr2. These findings could potentially be explained by the fact that all these proteins have a key role in post replicative repair and therefore are likely to harbour mutations in nuclear encoded Complex I genes not affecting mitochondrial copy number. This hypothesis is evidenced by the fact that the study by Mori et al examining frameshift mutations in $46 \mathrm{MSI}-\mathrm{H}$ tumours identified that in $28 \%$ there was a mutation in the nuclear encoded B14.5B Complex I subunit [380]. It is also important to note that there are several nuclear encoded genes, which are described to be essential in maintaining both mitochondrial and nuclear genomes. These include genes involved in non-homologous end joining such as Mre11-Rad50- Xrs2 and Yku70/80 complexes, the catalytic subunit of DNA polymerase $\zeta$ Rev3 involved in post replication repair and the BER enzymes Ogg1, Ntg1 glycosylases, as well as the APendonuclease Apn1. Deficiencies in these genes can lead to mtDNA damage and metabolic dysfunction. It Is therefore possible that MMR deficiency in general can lead to mutations within any of these essential genes resulting in decreased Complex I expression and activity due to their affect on the nuclear and mitochondrial genome encoding essential Complex I subunits [381]. 
There are also several studies supporting the role for $\mathrm{MSH} 2$ in the repair of oxidative DNA damage in the nucleus and possibly the mitochondria. Colussi et al and DeWeese et al have shown that when embryonic stem cells (ES) and mouse embryo fibroblasts (MEFs) from MSH2 deficient mice are compared to wildtype controls, these cells have higher baseline and ROS induced 8-oxoG lesions [219, 220]. Furthermore, Colussi et al showed that there were high levels of oxidative damage in MLH1 and MSH2 deficient cell lines due to defective removal of 8-oxoG that was present in DNA from the oxidized dNTP pool. The mutator phenotype observed in MSH2 deficient cell lines was reduced by the overexpression of the hydrolase MTHI which removes 8-oxodGTP [220]. We have shown that silencing of the nuclear encoded mitochondrial gene PINK1 is synthetically lethal with both MLH1 and MSH2 deficient cell lines due to an increase in oxidative DNA lesions in the nucleus and mitochondria of both these cell lines [97]. There are some studies contradicting a role for $\mathrm{MSH} 2$ in the mitochondria. Hashiguchi et al detected mismatch repair activity in $\mathrm{MSH} 2$ deficient cell lines concluding that $\mathrm{MSH} 2$ is unlikely to be involved in repair if DNA lesions in the mitochondria [244].

The observed phenotype in MSH2 deficient cell lines is similar to that seen in MLH1deficient cell lines. It is possible that the reasoning behind this observed phenotype is similar to MLH1-deficient cell lines with decreased expression of PGC1 $\beta$ and NRF1 resulting in decreased mitochondrial biogenesis, decreased expression and activity of Complex I and an attenuated antioxidant response. Due to time constraints, I have not established whether $\mathrm{MSH} 2$ deficient cell lines are synthetically lethal with Parthenoloide but it is likely that this is the case given the mitochondrial phenotype observed in these cell lines. We have previously shown that MSH2 deficient cell lines are selectively lethal with the oxidative DNA damaging agent Methotrexate which demonstrates that these cell lines are sensitive to oxidative DNA damage [98]. The ultimate reason for a decrease in cell viability with ROS inducing agents in these cell lines may be different to MLH1-deficient cell lines given that there is more evidence to attribute a role to MLH1 in the repair of oxidative DNA lesions in the mitochondria than MSH2 deficient cell lines. As described earlier there are numerous studies suggesting $\mathrm{MSH} 2$ has a role for repair of oxidative lesions in the nucleus and therefore an accumulation of oxidative DNA lesions in the nucleus may lead to decreased cell viability in MSH2 deficient cell lines. 


\subsection{DNA repair deficiency and mitochondrial metabolism}

A role for some form of MMR in the mitochondria is emerging through work from our lab and others. This opens the question about whether deficiency in other nuclear DNA repair proteins causes mitochondrial dysfunction either directly or indirectly.

\subsubsection{ATM and the mitochondria}

The DNA repair protein Ataxia-telangiectasia (ATM) is known to be a serine/threonine kinase recruited to sites of DNA damage resulting in DNA repair, apoptosis or cell cycle arrest $[382,383]$. ATM deficiency leads to the autosomal recessive disease ataxiatelangiectasia characterised by T-cell malignancies, cerebellar ataxia, insulin resistance, immune deficiency and premature ageing [384]. There is emerging evidence that the phenotype observed in ataxia-telangiectasia is unlikely to be solely related to the nuclear DNA repair functions of ATM and that ATM has other roles which have not been fully elucidated. Several studies have demonstrated that ATM deficiency is characterized by oxidative stress and sensitivity to ROS inducing agents [385, 386]. Ambrose et al investigated the link between ATM deficiency and high levels of oxidative stress by comparing wild type and A-T lymphoblastoid cells (ATM deficient). The investigators demonstrated that A-T cells had dysfunctional mitochondria compared to wild type cells as evidenced by abnormal mitochondrial structure, reduced mitochondrial membrane potential, increased levels of antioxidant enzymes, up regulation of mitochondrial targeted DNA repair proteins and decreased mitochondrial respiration [387]. Valentin-Vega et al demonstrated that in ATM deficient thymocytes isolated from mice the mitochondria were swollen with abnormal cristae as well as having increased mitochondrial ROS and decreased complex I activity [388]. The authors explored these findings further to try and elucidate whether ATM has a direct role in the mitochondria which is distinct from its involvement in the nuclear DNA damage response. They were able to show that ATM is present in the mitochondrial fraction of human fibroblasts and that upon treating these cells with the mitochondrial uncoupling agent CCCP, the ATM kinase is activated without a corresponding activation of the ATM substrates related to DNA damage [388]. Eaton et al showed that ATM signalling is involved in the regulation of the rate-limiting enzyme essential for the synthesis of deoxyribonucleoside triphosphates and mitochondrial homeostasis, ribonucleotide reductase (RR). They observed that primary fibroblasts deficient in ATM results in decreased expression of 
the R1, R2 and P53R2 subunit of RR with a corresponding impairment of the increase in mtDNA copy number that is normally observed in relation to ionizing radiation. The study concluded that ATM and its downstream target RR are essential in the control of mtDNA copy number in response to oxidative DNA damage and also in actively dividing cells [389].

There are two main hypotheses explaining the role of ATM in controlling mitochondrial homeostasis that have emerged. The first hypothesis proposes a direct involvement of ATM in controlling the redox state of the cell by exerting control of the antioxidant defence enzymes of the cell at a transcriptional or post-translational level [390]. Furthermore, there is some evidence to form the conclusion that ATM is directly activated upon sensing oxidative damage and could potentially phosphorylate mitochondrial proteins [96]. The indirect model is based on the premise that ATM deficient cells have lower levels of NAD+ due to the persistent DNA damage that is left unrepaired and the activation of repair enzymes such as PARP, which require NAD+ molecules. This leaves the cell with a reduced antioxidant capacity and increased ROS levels [391].

It is well established that when the MMR pathway is recruited to sites of DNA damage, MLH1 associates with ATM in recruiting other components of the DDR pathway [392]. Based on the studies mentioned above and the association of ATM with MLH1, it would be interesting to investigate whether the mitochondrial phenotype I have observed in my MLH1 deficient cell lines may be in part associated with ATM. It is possible that ATM may not be able to carry out its mitochondrial role in the absence of MLH1.

\subsubsection{DNA repair proteins and nucleus-to-mitochondria signalling}

Recent evidence suggests that nuclear DNA repair proteins and the nuclear DDR have an impact on mitochondrial function and that altered nucleus-to- mitochondria (NM) signalling contributes to ageing and age related disorders.[393-395]. This area of research has become a field of widespread interest.

There are several DNA repair proteins that have been found to be present in both the nucleus and the mitochondria including many of the BER proteins, RECQL4, petite 
integration frequent 1 (PIF1), DNA replication helicase/nuclease 2 (DNA2) and suppressor of Var 3-like protein 1 (SUPV3L1). These proteins are thought to not only protect nuclear DNA integrity but possibly have a role on maintaining mtDNA stability $[396,397]$. There are also several DNA repair proteins where it is unclear whether they are imported into the mitochondria under conditions of increased stress or have both nuclear and mitochondrial localization. Furthermore, several DNA repair proteins and pathways are not present in the mitochondria but signalling through these pathways has an effect on mitochondrial homeostasis [398] .

The primarily nuclear DNA repair protein RECQL4 is a DNA helicase which has been shown to localize to the mitochondria, be vital in the transport of p53 into the mitochondria and take part in mtDNA replication and repair [397]. Sengupta et al have shown that RECQL4 interacts (along with p53) with the mitochondrial polymerase PolyA/B2 [399]. P53 has been established to have a role in regulating mitochondrial function including biogenesis, mitophagy and the antioxidant response[400]. By carrying out mitochondrial genome sequencing on fibroblasts from patients with cancer predisposition syndromes due to RECQL4 and p53 deficiency (Rothmund-Thomson syndrome (RTS) and Li-Fraumeni syndrome (LFS) Rothmund, respectively) they were able to show increased somatic mutations and polymorphisms in mtDNA from these samples [401]These mutations have been found to be associated with ageing and cancer thereby eluding to the fact that the phenotypes seen in these syndromes could be due at least in part to mtDNA genome instability [396, 401]. Several lines of evidence have shown that DNA damage results in alterations to cellular metabolism, such that many diseases characterized by premature ageing result in weight loss [402]. An important pathway associating nuclear DNA damage to alterations in mitochondrial function is DNA damage leading to PARP1 activation and in turn alterations in the $\mathrm{NAD+/NADH}$ ratio of cells, which alters a multitude of metabolic processes. Evidence suggests that activation of the DNA damage response that leads to PARP1 activation leads to DNA repair signalling and a process known as PARylation which depletes the cells stores of NAD+ and acetyl-CoA which are essential for cell metabolism [403]. The consequence of NAD+ depletion is inhibition of the NAD+ dependent deacetylase enzymes known as the Sirtuins and alterations in the NAD+-SIRT1-PGC-1 axis [404]. These enzymes are implicated in the control of cell metabolism, ageing and epigenetic 
regulation through their role in modifying proteins and consuming NAD+ as a consequence [405]

It is clear from the evidence presented above that there is little doubt of the huge influence DNA repair pathways and the DDR proteins have on cell metabolism and mitochondrial function. The MMR pathway is a key DNA repair pathway and given the evidence presented here linking DNA damage to metabolism, it is not surprising that this study has established a potential link between the MMR protein MLH1 and mitochondrial metabolism. This link may potentially be associated with increased single strand breaks in the MLH1 deficient tumours leading to PARP1 activation, depletion of NAD+ and inhibition of the Sirtuin family resulting in the mitochondrial phenotype I observed. Furthermore, it is possible that in the context of MLH1 deficiency ATM cannot carry out its DDR role and activate AMPK and the downstream pathways including activating PGC1- $\alpha$ and FOXOs.

\subsection{ROS induction and targeting the redox capacity of cancer cells}

ROS are continuously being produced by enzymatic (NADPH oxidase, xanthine oxidase, endothelial nitric oxide synthase, arachidonic acid, cytochrome p450 enzymes, lipooxygenase and cyclooxygenase) and non-enzymatic reactions (respiratory chain ROS). ROS levels can be induced by factors such as ER stress [460], hypoxia [461], oncogenes [462] and metabolic defects [463]. On the other hand, ROS scavengers such as the antioxidant defense system [464] and tumour suppressors [465] can reduce ROS levels [113]. There are many research groups investigating the differences in the redox state of cancer cells compared to normal cells with the aim of developing ROS inducing agents as a therapeutic strategy in cancer. There is evidence to suggest that cancer cells have higher ROS levels compared to normal cells but the picture is obviously much more complex than this since the cancer cells can upregulate their antioxidant defense systems to cope with increased oxidative stress [466].

ROS biology in cancer is complex with some evidence suggesting these molecules cause cancer progression and others suggesting they can cause cancer cell death. It seems the response to ROS is dependent on a multitude of situational factors including the magnitude of the stress, the duration and the cell type affected. Current studies aim 
to find out under which circumstances these molecules act as tumour suppressors and when they act to cause tumour growth [467].

The first body of evidence linking ROS with increased cancer cell proliferation was carried out by Oberley et al showing that increased insulin caused raised levels of ROS and tumour proliferation [468]. Studies have shown that at low to moderate levels ROS can potentially act to facilitate tumour growth by causing oxidative stress and DNA mutations as well as acting directly or indirectly to affect the function of numerous enzymes, transcription factors and signaling pathways which in turn cause downstream genetic changes. ROS has been shown to modulate several pathways depending on the nature of the oxidative stress. Some of the pathways ROS can control include phosphorylating MAPK [469], ERK [470], cyclin D1 [471], ATM [472], NFkB [473], JNK activation [474] and inactivating tumour suppressors such as PTEN Moderate doses of ROS have been shown to cause temporary or more permanent cell cycle arrest and replicative senescence $[113,467]$.

Another consideration to bear in mind when considering using ROS inducing agents is the fact that evidence suggests that cancer stem cells or tumour initiating cells (TICs) have recently been found to have low ROS levels and increased antioxidant capacity [415]. This finding could possibly result in the TIC's being selectively spared since they are able to cope with increased levels of oxidative stress compared to the more mature cancer cells [415].

High levels of ROS and severe oxidative stress cause cell death but the final mechanism by which this occurs is still controversial. Some research groups have carried out studies suggesting that the mechanism is apoptosis and others suggest that the mitochondrial transition pore is involved $[113,475]$. Valencia et al have shown in in vitro that superoxide anions induce cell death in neuronal cells by apoptosis whereas hydrogen peroxide led to necrosis-like cell death [476].

There has also been a recent interest in studying ROS inducing agents as single treatments or in combination with conventional chemotherapy. These agents have been tested in early phase clinical trials but there are currently no drugs, aside from 
conventionally used chemotherapies with ROS generating properties, that have reached large scale phase III trials [364, 477].

\subsubsection{Chemotherapeutics as ROS inducing agents}

Several conventionally used chemotherapeutic agents are known to increase ROS and oxidative stress in addition to their main mechanism of action. The chemotherapies that are known to induce high levels of ROS include the anthracyclines, platinum agents, epipodophyllotoxins and the camptothecins [475]. ROS induction can either enhance or inhibit the efficacy chemotherapeutic agents $[478,479]$. The drug 5fu commonly used in CRC has also been shown to induce ROS through a p53 dependent pathway. A study examining 5-fu resistance in B cell lymphoma discovered that cancer cells that adapted to the oxidative stress by increasing antioxidant enzymes (SOD2 and peroxiredoxin) were found to be resistant to 5-fu [480].

\subsubsection{The quinones as ROS inducing agents}

In this study, I observed variable responses to the three ROS inducing agents Parthenolide, Menadione and $\beta$-lapachone. This observed difference could be due to the different mechanisms of action that these drugs have as outline below.

There are numerous small molecules which belong to different classes of ROS inducing agents that have been developed to overwhelm the antioxidant capacity of cancer cells and cause cell death. Two of the drugs used in this study, $\beta$-lapachone and Menadione are ROS inducing agents which belong to a family of drugs called quinones. These drugs all undergo futile redox cycles on the respiratory chain but $\beta$-Lapachone and Menadione have slightly different ways in which they carry out their mechanism of action

Menadione is a polycyclic aromatic ketone that is a vitamin k precursor [366]. It undergoes a one electron reduction by reductive enzymes including microsomal NADPH-cytochrome P450 reductase and mitochondrial NADH-ubiquinone oxidoreductase (Complex I) and the semiquinones formed as part of this reaction undergo futile redox cycling in the presence of molecular oxygen. Menadione has been found to exert its action through futile redox cycling on the respiratory chain thereby 
causing ROS production [366]. Menadione is a redox-dependant signalling molecule at low doses (e.g., $2 \mu \mathrm{M}$ ) but causes oxidative stress and cell death at higher doses [481]. Pre-clinical studies have shown Menadione to be an effective anti-cancer treatment [482] but no clinical trials to date have shown objective responses with this drug [483]. Given that my MLH1 deficient cell lines exhibit deficiencies in Complex I and that Menadione requires Complex I as part of its mechanism of action, this may explain why I did notobserve selective cell death in my MLH1 deficient cell lines with this ROS inducing agent

$\mathrm{NAD}(\mathrm{P}) \mathrm{H}$ :quinone oxidoreductase $1(\mathrm{NQO1})$ is another reductive enzyme which carries out two electron reduction reactions forming stable hydroquinones [484]. $\beta$-lapachone (clinical form ARQ761) is part of a group of novel quinones that undergo this reaction but form an unstable hydroquinone which in turn spontaneously undergoes oxidation consuming two oxygen molecules [485]. This reaction leads to futile redox cycling where one mole of $\beta$-lapachone leads to the formation of approximately 120 moles of superoxide within two minutes [486]. $\beta$-lapachone has been shown to be effective at killing cancer cells which overexpress NQO1 in experimental studies including a large proportion $(>80 \%)$ of non-small cell lung cancer cell lines [485], pancreatic [487] and breast cancer [485] cell lines. The mechanism of death in these cell lines has been shown to be due to the generation of superoxide anions causing DNA damage, ca2+ release from the endoplasmic reticulum resulting in PARP1 hyperactivation and NAD+/ATP loss and programmed cell necrosis [488, 489]. Bey et al explored the relationship of $\beta$-lapachone with the antioxidant system and established that catalase levels in addition to NQO1 levels was a major determining factor relating to the efficacy of this drug [486]. High levels of catalase and overexpression of this enzyme cause resistance to $\beta$-lapachone induced cell death [486]. ARQ761 is currently undergoing phase I clinical trials (ClinicalTrials.gov Identifier:NCT01502800) testing the maximum tolerated dose of the drug before moving into trials to assess efficacy I did not observe a synthetic lethal relationship with my MLH1 deficient cell line HCT116 and $\beta$ Lapachone compared to the MLH1 proficient cell line HCT116+chr3. This result could be due to the fact that these are isogentically matched cell lines and therefore are may have the same NQO1 levels and since $\beta$-Lapachone cell death has been shown to occur in an NQO1 dependent manner a difference would not be observed. 


\subsubsection{Parthenolide as a ROS inducing agent}

The sesquiterpene lactone Parthenolide is another ROS inducing agent used in this study. It is derived from the plan feverfew and it exerts its action through its lactone ring which reacts with cysteine thiol groups which are present on many regulatory proteins, kinases, phosphatases and transcription factors. Cysteine thiols through their oxidation and reduction have an essential role in facilitating signalling pathways due to redox changes [490]. The main mechanism of cell death of Parthenolide is ROS induction and oxidative stress [362].

Wen et al treated sarcomatoid hepatocellular carcinoma cells $(\mathrm{SH}-\mathrm{Jl})$ and hepatoma cells with Parthenolide and observed apoptosis of these cells at concentrations of 5-10 $\mu \mathrm{M}$ and cell cycle arrest at 1-3 $\mu \mathrm{M}$ concentrations [491]. As a consequence of Parthenolide induced apoptosis, the authors observed depletion of glutathione, generation of ROS, reduction of mitochondrial transmemebrane potential, activation of caspases and increased expression of the oxidative stress gene GADD153 [491]. An interesting study by Sun et al examined in detail the selective cell radiosensitization they observed with Parthenolide in the prostate cancer cell line PC3 compared to the normal prostate epithelial cell line prEC [362]. The authors concluded that in PC3 cells Parthenolide induces ROS by activating NADPH oxidase and also acts on the antioxidant capacity of these cells by decreasing levels of reduced thioredoxin, supressing FOXO3a (via activation of PI3K/AKt pathway) and its downstream targets SOD2 and catalase. In the PrEC cells Parthenolide did not activate NADPH oxidase and increased glutathione levels. The authors were able to shown that the PC3 and PrEC cells have different redox status' at baseline which was likely to account for the differences observed. The PC3 cells had higher Nox1 levels and lower SOD2 and catalase levels compared to the normal cells and Parthenolide caused further oxidative stress by inhibiting antioxidant enzymes and activating ROS. The normal cells had high GSH and GST levels at baseline and Parthenolide increased these levels further in the PrEC cells only. The reason behind the sparing of normal cells from Parthenolide cytotoxicity was thought to be due to the high GSH and GST levels which meant Parthenolide could conjugate with GSH facilitated by GST's and then be transported outside the cell leading to Parthenolide resistance [362]. This is a very relevant study in relation to my study since my MLH1 deficient cell line HCT116, like the PC3 prostate 
cancer cell line, demonstrates a decrease in antioxidant defence enzymes compared to the MLH1 proficient cell line HCT116+chr3. The selective cell death in my MLH1 deficient cell lines is likely to be due Parthenolide inhibiting the antioxidant defence enzymes in cells that already have deficiencies in these cell lines leading to increased ROS and cell death.

A study by Carlisi et al examined the different radical species produced during treatment of the human breast cancer cell line MDA-MB-231with Parthenolide [363]. They illustrated that during the first 1-8 hours after treatment NADPH oxidase was stimulated and there were high levels of $\mathrm{H} 2 \mathrm{O} 2$, during the second phase of treatment from 8-16 hours the main radical produced was mitochondrial O2- . Furthermore, the authors demonstrated that the levels of the antioxidant enzymes catalase and SOD2 decreased only after 4 hours of treatment and then gradually decreased over time after that with a $40 \%$ reduction at 16 hours. In my study I observed an increase in ROS within the first hour after treatment, using the dye DHE that detects superoxide anions. This is obviously different to the finding of Carlisi et al and may be due to differences in the cell lines used and experimental design such as the fact that they used flow cytometry to detect super oxide anions and I used the MitoXpress machine which is a fluorescent microscope. The short term cell viability assays carried out in this study were exposed to Parthenolide for five days which according to the study by Carlisi et al would be enough time to allow Parthenolide induced inhibition of the antioxidant defence enzymes. Parthenolide has entered phase I clinical trials which concluded that it has poor bioavailability [492] leading to a water-soluble analogue Dimethylamino Parthenolide (DMAPT) being developed. DMAPT has shown promising pre-clinical results in lung, bladder and breast cancer cell lines [364, 493].

From the evidence above it seems clear that Parthenolide induces ROS through acting on the antioxidant defence mechanism of cells. Menadione and $\beta$-lapachone are also ROS inducing agents but have a different mechanism of action to Parthenolide and have not been shown to act on the antioxidant system. It seems congruent therefore that Parthenolide has displayed a selective lethal relationship with my MLH1 deficient cell lines which have deficiencies in the antioxidant defence system and deregulated mitochondrial function. 


\subsubsection{Drugs inhibiting the antioxidant response}

Several drugs have also been developed to counteract the upregulation of the antioxidant defense system in cancer cells by indirectly increasing ROS levels. Glutathione metabolism is an important therapeutic drug target that has been identified and drugs such as Buthionine Sulphoximine, which inhibit GSH synthesis, have shown promise in experimental studies [494]. GSH levels can also be altered by modifying cysteine levels and the drug Sulphasalazine has been shown to be able to carry out this function and be effective in pancreatic cancer cell lines in vitro and in vivo [495]. The drug NOV-002 alters the GSSG/GSH ratio and has been shown to improve the response rate when added to conventional chemotherapy in the adjuvant setting in HER-2 negative breast cancers [496]. Alterations to the antioxidant enzyme thioredoxin is another resistance mechanism that cancer cells have been shown to employ and the drug Auranofin has been developed to counteract this. This drug is a gold compound that has been used for rheumatoid arthritis. It has been shown to cause cell death with cytochrome-c release in ovarian cancer cell lines [497] .

These therapeutics inhibiting the antioxidant defense system to modulate ROS levels have been developed some time ago but few have made progress beyond pre-clinical studies despite some promising results. The renewed interest in therapeutically targeting cancer cell metabolism should give the academic community a better understanding of cancer cell metabolism and hopefully move these drugs further forward into clinical trials.

\subsubsection{Synthetic lethal targeting of cancer cells with DNA repair deficiencies and ROS inducing agents}

With the increased move in oncology to try and specifically target cancer cells and spare normal body cells, several studies have been designed to specifically target cells with DNA repair deficiencies by introducing ROS and oxidative stress in these cell lines

Sajesh et al manipulated the redox status of cancer cells deficient in the homologous recombination DNA repair enzyme RAD54B [498]. The investigators found that inhibiting the antioxidant enzyme SOD1 using the novel therapeutic ATTM and by 
siRNA silencing results in selective cell death in the RAD54B deficient cell line $(E C 50=4.2 \mu \mathrm{M})$ compared to controls $(E C 50=67.7 \mu \mathrm{M})$. On further investigation, the authors show that the mechanism of cell death is due to a significantly increased accumulation of DNA double strand breaks due to increased ROS levels in the RAD54B-deficient colorectal cancer cell lines leading to apoptosis in these cell lines compared to controls. The authors discussed several possible reasons behind the selectivity including the fact that the damage caused by inhibiting SOD1 could be of a type (S-phase as a result of collapsed replication forks) that can only be repaired by the homologous recombination pathway and not compensated for by non homologous end joining. Other potential hypotheses include that ROS induction activates pathways that suppresses compensatory non homologous end joining or that the damage overwhelms the capacity of the non homologous end joining pathway [498].

A recent study by Chakrabati et al exploited the mechanism of action of ARQ761 ( $\beta$ Lapachone) and demonstrated that knocking down the BER scaffolding protein XRCC1 sensitised cells to PARP1 hyperactivation with ARQ761 treatment resulting in enhanced cell killing in an NQO1 dependent manner in head and neck cell lines, breast cancer cell lines, non small cell lung cancer and pancreatic ductal adenocarcinoma [368]. On further exploring the observed selective cell death, the investigators observed that in pancreatic cancer tissue the levels of NQO1 were much higher compared to normal tissue. Interestingly, raised NQO1 levels in pancreatic cancer tissue were accompanied by decreased expression of the antioxidant enzyme Catalase. The investigators demonstrated that exposing pancreatic ductal adenocarcinoma (PDA) MiaPaca2 cell lines to ARQ761 resulted in a NQO1 dependent increase in cytoplasmic ROS, DNA base damage and single strand breaks but no significant cell death [368]. Upon treating several different cell lines with sublethal doses of $A R Q 761$ and the DNA repair inhibitor MeOx (binds to AP sites and prevents recruitment of BER complexes to damage [499]) this led to enhanced cell death in the cancer cell lines in an NQO1 dependent manner due to increased ARQ761 induced PARP1 hyperactivation. The overall mechanism by which the authors described cell death was occurring is that MeOx causes modification of AP sites means that there is already increased PARP1 activation and depletion of NAD+ which means that further damage by ARQ761 results in PARP1 not being able to be activated and repair the resulting single strand breaks. Furthermore, in vivo in mice bearing MiaPaca2- derived 
xenografts there was a significant decrease in tumour growth and increased survival in the mice treated with the combination of ARQ761 and MeOx [368].

My study has demonstrated that cells deficient in the MMR gene MLH1 have a mitochondrial phenotype, a decreased antioxidant defense capacity and a synthetic lethal relationship with the ROS inducing drug Pathenolide. In a similar manner to the studies described above, it is likely that the mechanism of selectivity is related to the DNA repair function of MLH1. As outlined in section previously, there is evidence to suggest that the MMR repair system has a role in the repair of oxidative DNA damage and treatment with the ROS inducing agent Parthenolide results in increased ROS in my MLH1 deficient cell lines compared to the MLH1 proficient cell lines and this is likely to lead to oxidative DNA damage that the MLH1 deficient cell lines are not able to repair.

\subsection{Targeting MMR deficient cell lines}

Dwyer et al have recently shown that several conventionally used antibiotics produce ROS as part of their mechanism of action despite having differing mechanisms by which they carry out their bactericidal activity [500]. The role of ROS in antibiotic lethality was demonstrated by overexpressing the H202 scavenging enzyme KatG in cell culture with several antibiotics which resulted in decreased cell killing [500]. The role of the MutS was also investigated in this study such that overexpressing this MMR protein resulted in decreased antibiotic lethality. The authors concluded that the post replicative repair function of MMR was likely to be responsible for this but that other novel roles of MMR pathway in this context could not be ruled out [500]. It is feasible that the role of MMR in the repair of oxidative DNA damage could also be contributing to the increased cell survival. It would be interesting to investigate whether MMR deficient cells are synthetically lethal with the antibiotics used in this study and whether this is as a result of increased oxidative damage.

Our laboratory has previously shown that treatment with the drug Methotrexate is selectively lethal with $\mathrm{MSH} 2$ deficient cell lines, due to an increased susceptibility to 
oxidative stress [98]. The levels of 8-hydroxyguanine (8-OHG), a precursor to 8-oxoG, were similar in the $\mathrm{MSH} 2$ proficient and deficient cell lines following methotrexate treatment, but over time the levels of $8-\mathrm{OHG}$ returned to baseline in the $\mathrm{MSH} 2$ proficient cell lines. Levels of oxidative DNA damage remained elevated in the $\mathrm{MSH} 2$ deficient cell lines. This data suggests that in the absence of $\mathrm{MSH} 2$, oxidative DNA damaging agents such as Methotrexate can cause oxidative damage, which is not efficiently repaired and its accumulation results in loss of cell viability. These findings have been translated into an ongoing clinical trial at the Royal Marsden Hospital [98]. More recently, it has been shown that cytosine based nucleoside analogs are selectively lethal with MLH1 and MSH2 deficient cell lines due to an increase in ROS levels and elevated oxidative DNA damage leading to apoptosis [52].

The evidence presented here clearly highlights that targeting DNA repair deficient cells like the MMR deficient cell lines in this study with ROS inducing agents is a useful therapeutic strategy and warrants further study.

There are obviously concerns about normal tissue damage with mitochondrial targeted agents especially ROS inducing drugs given the crucial role of ROS in signaling pathways in normal tissue physiology. Interfering with mitochondrial ATP production in cancer cells may have the unintended consequence of affecting ATP production in normal cells. A large amount of research is ongoing to try and develop an in-depth understanding of the differences between the mitochondria and redox status of cancer cells versus normal cells and indeed more recently that of cancer stem cells. This type of research will allow more selective targeting of cancer cells versus normal cells with mitochondrial- targeted drugs. There is a also a move to develop therapeutics to specifically target the mitochondria with the hope to improve efficacy and reduce toxicity. Direct targeting of the mitochondria is thought to have the advantage of avoiding any upstream pathways that may be involved in drug resistance. Methods of direct mitochondrial targeting that have been developed include: 1, Delocalized lipophilic cations (DLCs) which cross the mitochondrial membranes since they are designed to target the negative charge of the matrix [501]. 2, mitochondrial targeted sequences (MTSs)- containing polypeptides with amino acids designed to be recognized by the mitochondrial import machinery [502]. 3, Synthetic amino acid and peptide based mitochondrial transporters are made in such a way that they enter the 
cell directly followed by entering the mitochondria facilitated by the electrical gradient and therefore avoid lysosomal or endosomal degradation [503]. 4, Mitochondriatargeted liposomes [504]. 


\subsection{Clinical relevance}

We and others have previously established a role for the MMR pathway in the repair of oxidative DNA damage in the nucleus (MSH2) and the mitochondria (MLH1). We have also shown MLH1 to be synthetically lethal with several mitochondrial-targeted drugs due to an increase in oxidative DNA damage in the mitochondria. This study has deepened our understanding of MMR deficient tumours further and identified several potential therapeutic targets.

More specifically I have shown that MLH1-deficient cancers (and potentially MSH2 deficient cancers) could be selectively targeted by exploiting the fact that they are susceptible to the ROS inducing agent Parthenolide because of deficiencies in their antioxidant defense capacity compounded by the fact that these cell lines cannot repair the resulting mitochondrial oxidative DNA damage (Figure 6.1A).

I have identified the drug Parthenolide to be selectively lethal with MMR deficient tumours and further in vivo experiments need to be carried out to validate this before potentially moving into clinical trials. The plant Feverfew (Tanacet trade mark ) containing Parthenolide underwent a phase I clinical trial but was found to have poor bioavailability [381]. The drug has been re-formulated as a water soluble analogue known as Dimethylamino Parthenolide (DMAPT) and is currently undergoing phase I-II clinical trials. Interestingly Hassane et al, through chemical genomic studies, identified inhibitors of the PI3K/mTOR pathway which are known to interfere with the activation of NRF2 to work synergistically with DMAPT resulting in increased cell death in in vitro and in vivo experiments with primary human AML cells. [382], [383].

There is much debate about using ROS inducing agents and drugs that cause oxidative DNA damage in cancer since they could potentially enhance tumourigenesis. It is important to note that several conventionally used chemotherapeutic agents such as the anthracycline group of drugs and methotrexate are know to induce ROS and cause oxidative DNA damage as one of their main mechanisms of action [188, 384]. These drugs have been given safely by having a good understanding of their potential toxicities and devising strategies to monitor these side effects. Furthermore, the main 
advantage of targeted strategies like I have identified in this study is that these should in theory selectively target the MMR deficient tumour whilst sparing normal body cells.

\section{Figure 6.1}

A

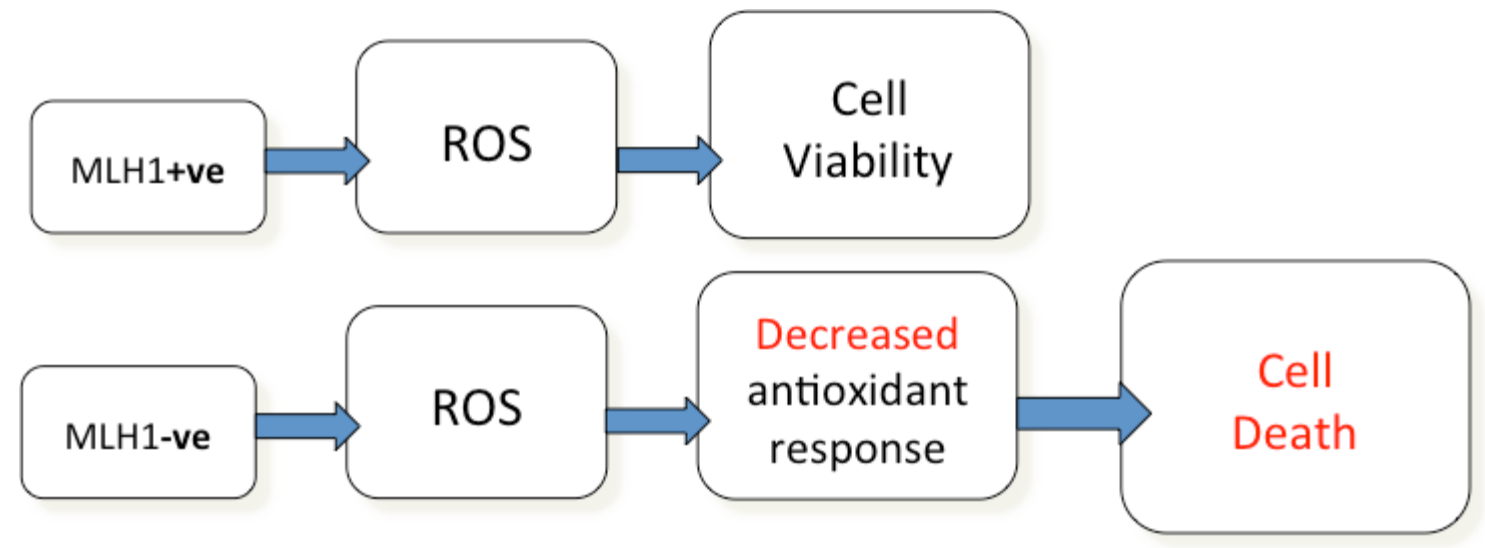

Figure 6.1. (A) MLH1-deficient cell lines can be exploited clinically due to an attenuated antioxidant response. 


\subsection{Conclusions}

This study has shown for the first time that MMR deficient cell lines (MLH1 and MSH2 deficient) exhibit a complex mitochondrial phenotype that could be exploited clinically. These cell lines have decreased expression of $P G C 1 \beta, N R F$, decreased mitochondrial biogenesis, decreased expression of the transcription factor NRF2 and this results in deficiencies in Complex I expression and activity, dysfunctional oxidative phosphorylation and an attenuated antioxidant response system (Figure 7.1A). 
Figure 7.1

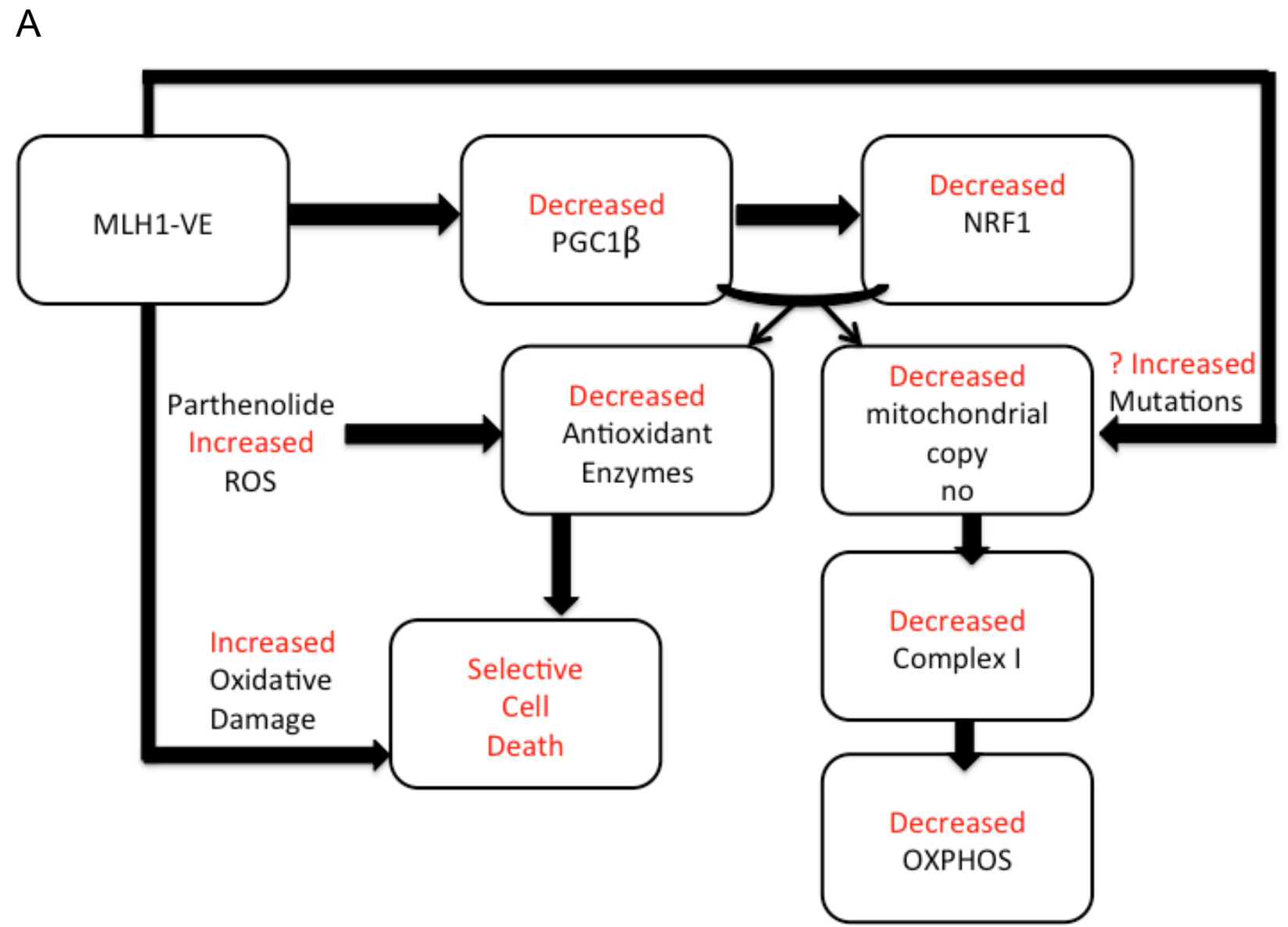

Figure 7.1 MLH1-deficient cell lines display a complex mitochondrial phenotype.

(A) MLH1-deficient cell lines have decreased expression of the master co-regulator PGC1- $\beta$ and the transcription factor NRF1. This results in decreased mitochondrial biogenesis, deficiencies in Complex I, decreased oxidative phosphorylation and an attenuated antioxidant system. Treatment with the mitochondrial-targeted agent Parthenolide results in a synthetic lethal relationship and selective cell death in the MLH1-deficient cell lines because of the attenuated antioxidant system and the inability of MLH1-deficient cell lines to repair the resulting oxidative DNA damage. 


\subsection{Future work}

To gain a full understanding of the phenotype I observed in my MLH1 and MSH2 deficient tumours, we need to understand why and how these tumours have deficiencies in PGC1 $\beta$ and downstream transcription factors. As a starting point, we could sequence these genes looking for mutations within microsatellites in their sequences. The fact that all the MMR deficient cell lines in this study exhibited decreased Complex I expression and activity means that it is possible they have mutations within microsatellites in Complex I genes or nuclear genes involved in mtDNA integrity. To investigate this we could sequence nuclear encoded Complex I genes as well as other key nuclear encoded mitochondrial genes such as TFAM.

The next step towards translating the results of this study would be to carry out an in vivo experiment with the drug Parthenolide in mice xenografted with MLH1-deficient and proficient tumour cells. 


\section{Bibliography}

1. Lord, C.J. and A. Ashworth, The DNA damage response and cancer therapy. Nature, 2012. 481(7381): p. 287-94.

2. Martin, S.A., C.J. Lord, and A. Ashworth, DNA repair deficiency as a therapeutic target in cancer. Curr Opin Genet Dev, 2008. 18(1): p. 80-6.

3. Tuppen, H.A., et al., Mitochondrial DNA mutations and human disease. Biochim Biophys Acta, 2010. 1797(2): p. 113-28.

4. Schon, E.A., S. DiMauro, and M. Hirano, Human mitochondrial DNA: roles of inherited and somatic mutations. Nat Rev Genet, 2012. 13(12): p. 878-90.

5. Park, J.S., et al., A heteroplasmic, not homoplasmic, mitochondrial DNA mutation promotes tumorigenesis via alteration in reactive oxygen species generation and apoptosis. Hum Mol Genet, 2009. 18(9): p. 1578-89.

6. $\quad$ Li, G.M., Mechanisms and functions of DNA mismatch repair. Cell Res, 2008. 18(1): p. 85-98.

7. Genschel, J., et al., Isolation of MutSbeta from human cells and comparison of the mismatch repair specificities of MutSbeta and MutSalpha. J Biol Chem, 1998. 273(31): p. 19895-901.

8. Kolodner, R.D. and G.T. Marsischky, Eukaryotic DNA mismatch repair. Curr Opin Genet Dev, 1999. 9(1): p. 89-96.

9. Marra, G. and P. Schar, Recognition of DNA alterations by the mismatch repair system. Biochem J, 1999. 338 ( Pt 1): p. 1-13.

10. Alani, E., The Saccharomyces cerevisiae Msh2 and Msh6 proteins form a complex that specifically binds to duplex oligonucleotides containing mismatched DNA base pairs. Mol Cell Biol, 1996. 16(10): p. 5604-15.

11. Plotz, G., et al., N-terminus of hMLH1 confers interaction of hMutLalpha and hMutLbeta with hMutSalpha. Nucleic Acids Res, 2003. 31(12): p. 3217-26.

12. Kunkel, T.A. and D.A. Erie, DNA mismatch repair. Annu Rev Biochem, 2005. 74: p. 681-710.

13. Jiricny, J., The multifaceted mismatch-repair system. Nat Rev Mol Cell Biol, 2006. 7(5): p. 335-46.

14. Plotz, G., et al., hMutSalpha forms an ATP-dependent complex with hMutLalpha and hMutLbeta on DNA. Nucleic Acids Res, 2002. 30(3): p. 711-8.

15. Martin, S.A., C.J. Lord, and A. Ashworth, Therapeutic targeting of the DNA mismatch repair pathway. Clin Cancer Res, 2010. 16(21): p. 5107-13.

16. Cannavo, E., et al., Expression of the MutL homologue hMLH3 in human cells and its role in DNA mismatch repair. Cancer Res, 2005. 65(23): p. 10759-66.

17. Sharma, A., et al., Slow conformational changes in MutS and DNA direct ordered transitions between mismatch search, recognition and signaling of DNA repair. $\mathrm{J}$ Mol Biol, 2013. 425(22): p. 4192-205.

18. Holmes, J., Jr., S. Clark, and P. Modrich, Strand-specific mismatch correction in nuclear extracts of human and Drosophila melanogaster cell lines. Proc Natl Acad Sci U S A, 1990. 87(15): p. 5837-41.

19. Claverys, J.P. and S.A. Lacks, Heteroduplex deoxyribonucleic acid base mismatch repair in bacteria. Microbiol Rev, 1986. 50(2): p. 133-65. 
20. Pluciennik, A., et al., PCNA function in the activation and strand direction of MutLalpha endonuclease in mismatch repair. Proc Natl Acad Sci U S A, 2010. 107(37): p. 16066-71.

21. Ghodgaonkar, M.M., et al., Ribonucleotides misincorporated into DNA act as strand-discrimination signals in eukaryotic mismatch repair. Mol Cell, 2013. 50(3): p. 323-32.

22. Masih, P.J., D. Kunnev, and T. Melendy, Mismatch Repair proteins are recruited to replicating DNA through interaction with Proliferating Cell Nuclear Antigen (PCNA). Nucleic Acids Res, 2008. 36(1): p. 67-75.

23. Kelman, Z. and J. Hurwitz, Protein-PCNA interactions: a DNA-scanning mechanism? Trends Biochem Sci, 1998. 23(7): p. 236-8.

24. Jiang, J., et al., Detection of high-affinity and sliding clamp modes for MSH2MSH6 by single-molecule unzipping force analysis. Mol Cell, 2005. 20(5): p. 77181.

25. Fishel, R., Mismatch repair, molecular switches, and signal transduction. Genes Dev, 1998. 12(14): p. 2096-101.

26. McNally, R., et al., Analysis of the role of PCNA-DNA contacts during clamp loading. BMC Struct Biol, 2010. 10: p. 3.

27. Dzantiev, L., et al., A defined human system that supports bidirectional mismatch-provoked excision. Mol Cell, 2004. 15(1): p. 31-41.

28. Ramilo, C., et al., Partial reconstitution of human DNA mismatch repair in vitro: characterization of the role of human replication protein A. Mol Cell Biol, 2002. 22(7): p. 2037-46.

29. Zhang, Y., et al., Reconstitution of 5'-directed human mismatch repair in a purified system. Cell, 2005. 122(5): p. 693-705.

30. Genschel, J. and P. Modrich, Functions of MutLalpha, replication protein A (RPA), and HMGB1 in 5'-directed mismatch repair. J Biol Chem, 2009. 284(32): p. 21536-44.

31. Kuriyan, J. and M. O'Donnell, Sliding clamps of DNA polymerases. J Mol Biol, 1993. 234(4): p. 915-25.

32. Li, G.M. and P. Modrich, Restoration of mismatch repair to nuclear extracts of H6 colorectal tumor cells by a heterodimer of human MutL homologs. Proc Natl Acad Sci U S A, 1995. 92(6): p. 1950-4.

33. Habraken, Y., et al., ATP-dependent assembly of a ternary complex consisting of a DNA mismatch and the yeast MSH2-MSH6 and MLH1-PMS1 protein complexes. J Biol Chem, 1998. 273(16): p. 9837-41.

34. Blackwell, L.J., S. Wang, and P. Modrich, DNA chain length dependence of formation and dynamics of hMutSalpha.hMutLalpha.heteroduplex complexes. J Biol Chem, 2001. 276(35): p. 33233-40.

35. Junop, M.S., et al., In vitro and in vivo studies of MutS, MutL and MutH mutants: correlation of mismatch repair and DNA recombination. DNA Repair (Amst), 2003. 2(4): p. 387-405.

36. Prolla, T.A., et al., Tumour susceptibility and spontaneous mutation in mice deficient in Mlh1, Pms1 and Pms2 DNA mismatch repair. Nat Genet, 1998. 18(3): p. 276-9.

37. Peltomaki, P., Lynch syndrome genes. Fam Cancer, 2005. 4(3): p. 227-32.

38. Ellison, A.R., J. Lofing, and G.A. Bitter, Human MutL homolog (MLH1) function in DNA mismatch repair: a prospective screen for missense mutations in the ATPase domain. Nucleic Acids Res, 2004. 32(18): p. 5321-38. 
39. Plotz, G., et al., Mutations in the MutSalpha interaction interface of MLH1 can abolish DNA mismatch repair. Nucleic Acids Res, 2006. 34(22): p. 6574-86.

40. Nagasaka, T., et al., Colorectal cancer with mutation in BRAF, KRAS, and wildtype with respect to both oncogenes showing different patterns of DNA methylation. J Clin Oncol, 2004. 22(22): p. 4584-94.

41. Jass, J.R., HNPCC and sporadic MSI-H colorectal cancer: a review of the morphological similarities and differences. Fam Cancer, 2004. 3(2): p. 93-100.

42. Ban, C. and W. Yang, Crystal structure and ATPase activity of MutL: implications for DNA repair and mutagenesis. Cell, 1998. 95(4): p. 541-52.

43. Spampinato, C. and P. Modrich, The MutL ATPase is required for mismatch repair. J Biol Chem, 2000. 275(13): p. 9863-9.

44. Polosina, Y.Y. and C.G. Cupples, MutL: conducting the cell's response to mismatched and misaligned DNA. Bioessays, 2010. 32(1): p. 51-9.

45. Kadyrov, F.A., et al., Endonucleolytic function of MutLalpha in human mismatch repair. Cell, 2006. 126(2): p. 297-308.

46. Kadyrov, F.A., et al., Saccharomyces cerevisiae MutLalpha is a mismatch repair endonuclease. J Biol Chem, 2007. 282(51): p. 37181-90.

47. Qiu, R., et al., MutL traps MutS at a DNA mismatch. Proc Natl Acad Sci U S A, 2015. 112(35): p. 10914-9.

48. Chambers, S.R., et al., The mismatch repair system reduces meiotic homeologous recombination and stimulates recombination-dependent chromosome loss. Mol Cell Biol, 1996. 16(11): p. 6110-20.

49. de Wind, N., et al., Inactivation of the mouse Msh2 gene results in mismatch repair deficiency, methylation tolerance, hyperrecombination, and predisposition to cancer. Cell, 1995. 82(2): p. 321-30.

50. Selva, E.M., et al., Mismatch correction acts as a barrier to homeologous recombination in Saccharomyces cerevisiae. Genetics, 1995. 139(3): p. 1175-88.

51. Kunz, C. and P. Schar, Meiotic recombination: sealing the partnership at the junction. Curr Biol, 2004. 14(22): p. R962-4.

52. Snowden, T., et al., hMSH4-hMSH5 adenosine nucleotide processing and interactions with homologous recombination machinery. J Biol Chem, 2008. 283(1): p. 145-54.

53. Worth, L., Jr., et al., Mismatch repair proteins MutS and MutL inhibit RecAcatalyzed strand transfer between diverged DNAs. Proc Natl Acad Sci U S A, 1994. 91(8): p. 3238-41.

54. Ray, B.L., C.I. White, and J.E. Haber, Heteroduplex formation and mismatch repair of the "stuck" mutation during mating-type switching in Saccharomyces cerevisiae. Mol Cell Biol, 1991. 11(10): p. 5372-80.

55. Alani, E., et al., Saccharomyces cerevisiae MSH2, a mispaired base recognition protein, also recognizes Holliday junctions in DNA. J Mol Biol, 1997. 265(3): p. 289-301.

56. Allers, T. and M. Lichten, Intermediates of yeast meiotic recombination contain heteroduplex DNA. Mol Cell, 2001. 8(1): p. 225-31.

57. Lipkin, S.M., et al., Meiotic arrest and aneuploidy in MLH3-deficient mice. Nat Genet, 2002. 31(4): p. 385-90.

58. Radman, M., Mismatch repair and the fidelity of genetic recombination. Genome, 1989. 31(1): p. 68-73.

59. Datta, A., et al., Dual roles for DNA sequence identity and the mismatch repair system in the regulation of mitotic crossing-over in yeast. Proc Natl Acad Sci U S A, 1997. 94(18): p. 9757-62. 
60. Nicholson, A., et al., Regulation of mitotic homeologous recombination in yeast. Functions of mismatch repair and nucleotide excision repair genes. Genetics, 2000. 154(1): p. 133-46.

61. Reitmair, A.H., et al., MSH2 deficient mice are viable and susceptible to lymphoid tumours. Nat Genet, 1995. 11(1): p. 64-70.

62. Chahwan, R., et al., AIDing antibody diversity by error-prone mismatch repair. Semin Immunol, 2012. 24(4): p. 293-300.

63. Rada, C., J.M. Di Noia, and M.S. Neuberger, Mismatch recognition and uracil excision provide complementary paths to both Ig switching and the A/T-focused phase of somatic mutation. Mol Cell, 2004. 16(2): p. 163-71.

64. Wiesendanger, M., et al., Somatic hypermutation in MutS homologue (MSH)3-, MSH6-, and MSH3/MSH6-deficient mice reveals a role for the MSH2-MSH6 heterodimer in modulating the base substitution pattern. J Exp Med, 2000. 191(3): p. 579-84.

65. Bardwell, P.D., et al., Altered somatic hypermutation and reduced class-switch recombination in exonuclease 1-mutant mice. Nat Immunol, 2004. 5(2): p. 224-9.

66. Wilson, T.M., et al., MSH2-MSH6 stimulates DNA polymerase eta, suggesting a role for A:T mutations in antibody genes. J Exp Med, 2005. 201(4): p. 637-45.

67. Kadyrova, L.Y., E.R. Blanko, and F.A. Kadyrov, CAF-I-dependent control of degradation of the discontinuous strands during mismatch repair. Proc Natl Acad Sci U S A, 2011. 108(7): p. 2753-8.

68. Schopf, B., et al., Interplay between mismatch repair and chromatin assembly. Proc Natl Acad Sci U S A, 2012. 109(6): p. 1895-900.

69. Kadyrova, L.Y., et al., A reversible histone H3 acetylation cooperates with mismatch repair and replicative polymerases in maintaining genome stability. PLoS Genet, 2013. 9(10): p. e1003899.

70. Richter, C., Oxidative damage to mitochondrial DNA and its relationship to ageing. Int J Biochem Cell Biol, 1995. 27(7): p. 647-53.

71. Hudson, E.K., et al., Age-associated change in mitochondrial DNA damage. Free Radic Res, 1998. 29(6): p. 573-9.

72. Svilar, D., et al., Base excision repair and lesion-dependent subpathways for repair of oxidative DNA damage. Antioxid Redox Signal, 2011. 14(12): p. 2491507.

73. Hashiguchi, K., V.A. Bohr, and N.C. de Souza-Pinto, Oxidative stress and mitochondrial DNA repair: implications for NRTIs induced DNA damage. Mitochondrion, 2004. 4(2-3): p. 215-22.

74. Mason, K.D., et al., The BH3 mimetic compound, ABT-737, synergizes with a range of cytotoxic chemotherapy agents in chronic lymphocytic leukemia. Leukemia, 2009. 23(11): p. 2034-41.

75. de Souza-Pinto, N.C., et al., Novel DNA mismatch-repair activity involving YB-1 in human mitochondria. DNA Repair (Amst), 2009. 8(6): p. 704-19.

76. Habano, W., S. Nakamura, and T. Sugai, Microsatellite instability in the mitochondrial DNA of colorectal carcinomas: evidence for mismatch repair systems in mitochondrial genome. Oncogene, 1998. 17(15): p. 1931-7.

77. Chi, N.W. and R.D. Kolodner, Purification and characterization of MSH1, a yeast mitochondrial protein that binds to DNA mismatches. J Biol Chem, 1994. 269(47): p. 29984-92.

78. Mishra, M. and R.A. Kowluru, Retinal mitochondrial DNA mismatch repair in the development of diabetic retinopathy, and its continued progression after 
termination of hyperglycemia. Invest Ophthalmol Vis Sci, 2014. 55(10): p. 69607.

79. Martin, S.A., et al., DNA polymerases as potential therapeutic targets for cancers deficient in the DNA mismatch repair proteins MSH2 or MLH1. Cancer Cell, 2010. 17(3): p. 235-48.

80. Kanzaki, H., et al., The association between RAD18 Arg302GIn polymorphism and the risk of human non-small-cell lung cancer. J Cancer Res Clin Oncol, 2008. 134(2): p. 211-7.

81. Popat, S., R. Hubner, and R.S. Houlston, Systematic review of microsatellite instability and colorectal cancer prognosis. J Clin Oncol, 2005. 23(3): p. 609-18.

82. Resnick, K.E., et al., Mismatch repair status and outcomes after adjuvant therapy in patients with surgically staged endometrial cancer. Gynecol Oncol, 2010. 117(2): p. 234-8.

83. Xiao, X., D.W. Melton, and C. Gourley, Mismatch repair deficiency in ovarian cancer -- molecular characteristics and clinical implications. Gynecol Oncol, 2014. 132(2): p. 506-12.

84. Hewish, M., et al., Cytosine-based nucleoside analogs are selectively lethal to DNA mismatch repair-deficient tumour cells by enhancing levels of intracellular oxidative stress. Br J Cancer, 2013. 108(4): p. 983-92.

85. Hewish, M., et al., Mismatch repair deficient colorectal cancer in the era of personalized treatment. Nat Rev Clin Oncol, 2010. 7(4): p. 197-208.

86. Lynch, H.T., et al., Hereditary colorectal cancer syndromes: molecular genetics, genetic counseling, diagnosis and management. Fam Cancer, 2008. 7(1): p. 2739.

87. Cohen, S.A. and A. Leininger, The genetic basis of Lynch syndrome and its implications for clinical practice and risk management. Appl Clin Genet, 2014. 7: p. 147-58.

88. Bhattacharyya, N.P., et al., Mutator phenotypes in human colorectal carcinoma cell lines. Proc Natl Acad Sci U S A, 1994. 91(14): p. 6319-23.

89. Parsons, R., et al., Hypermutability and mismatch repair deficiency in RER+ tumor cells. Cell, 1993. 75(6): p. 1227-36.

90. Jacob, S. and F. Praz, DNA mismatch repair defects: role in colorectal carcinogenesis. Biochimie, 2002. 84(1): p. 27-47.

91. Gatalica, Z. and E. Torlakovic, Pathology of the hereditary colorectal carcinoma. Fam Cancer, 2008. 7(1): p. 15-26.

92. Umar, A., et al., Revised Bethesda Guidelines for hereditary nonpolyposis colorectal cancer (Lynch syndrome) and microsatellite instability. J Natl Cancer Inst, 2004. 96(4): p. 261-8.

93. Boland, C.R., et al., A National Cancer Institute Workshop on Microsatellite Instability for cancer detection and familial predisposition: development of international criteria for the determination of microsatellite instability in colorectal cancer. Cancer Res, 1998. 58(22): p. 5248-57.

94. Berg, K.D., et al., Detection of microsatellite instability by fluorescence multiplex polymerase chain reaction. J Mol Diagn, 2000. 2(1): p. 20-8.

95. Laghi, L., P. Bianchi, and A. Malesci, Differences and evolution of the methods for the assessment of microsatellite instability. Oncogene, 2008. 27(49): p. 631321.

96. Devaud, N. and S. Gallinger, Chemotherapy of MMR-deficient colorectal cancer. Fam Cancer, 2013. 12(2): p. 301-6. 
97. Sankila, R., et al., Better survival rates in patients with MLH1-associated hereditary colorectal cancer. Gastroenterology, 1996. 110(3): p. 682-7.

98. Li, L.S., et al., DNA mismatch repair (MMR)-dependent 5-fluorouracil cytotoxicity and the potential for new therapeutic targets. Br J Pharmacol, 2009. 158(3): $\mathrm{p}$. 679-92.

99. Liu, A., et al., The mismatch repair-mediated cell cycle checkpoint response to fluorodeoxyuridine. J Cell Biochem, 2008. 105(1): p. 245-54.

100. Meyers, M., et al., A role for DNA mismatch repair in sensing and responding to fluoropyrimidine damage. Oncogene, 2003. 22(47): p. 7376-88.

101. Wolmark, N., et al., The benefit of leucovorin-modulated fluorouracil as postoperative adjuvant therapy for primary colon cancer: results from National Surgical Adjuvant Breast and Bowel Project protocol C-03. J Clin Oncol, 1993. 11(10): p. 1879-87.

102. O'Connell, M.J., et al., Controlled trial of fluorouracil and low-dose leucovorin given for 6 months as postoperative adjuvant therapy for colon cancer. J Clin Oncol, 1997. 15(1): p. 246-50.

103. Andre, T., et al., Improved overall survival with oxaliplatin, fluorouracil, and leucovorin as adjuvant treatment in stage II or III colon cancer in the MOSAIC trial. J Clin Oncol, 2009. 27(19): p. 3109-16.

104. Labianca, R., et al., Early colon cancer: ESMO Clinical Practice Guidelines for diagnosis, treatment and follow-up. Ann Oncol, 2013. 24 Suppl 6: p. vi64-72.

105. Quasar Collaborative, G., et al., Adjuvant chemotherapy versus observation in patients with colorectal cancer: a randomised study. Lancet, 2007. 370(9604): p. 2020-9.

106. Ribic, C.M., et al., Tumor microsatellite-instability status as a predictor of benefit from fluorouracil-based adjuvant chemotherapy for colon cancer. $\mathrm{N}$ Engl J Med, 2003. 349(3): p. 247-57.

107. Sargent, D.J., et al., Defective mismatch repair as a predictive marker for lack of efficacy of fluorouracil-based adjuvant therapy in colon cancer. J Clin Oncol, 2010. 28(20): p. 3219-26.

108. Sinicrope, F.A., et al., DNA mismatch repair status and colon cancer recurrence and survival in clinical trials of 5-fluorouracil-based adjuvant therapy. J Natl Cancer Inst, 2011. 103(11): p. 863-75.

109. Meyers, M., et al., Role of the hMLH1 DNA mismatch repair protein in fluoropyrimidine-mediated cell death and cell cycle responses. Cancer Res, 2001. 61(13): p. 5193-201.

110. Tejpar, S., et al., Microsatellite instability, prognosis and drug sensitivity of stage II and III colorectal cancer: more complexity to the puzzle. J Natl Cancer Inst, 2011. 103(11): p. 841-4.

111. Hutchins, G., et al., Value of mismatch repair, KRAS, and BRAF mutations in predicting recurrence and benefits from chemotherapy in colorectal cancer. J Clin Oncol, 2011. 29(10): p. 1261-70.

112. Klingbiel, D., et al., Prognosis of stage II and III colon cancer treated with adjuvant 5-fluorouracil or FOLFIRI in relation to microsatellite status: results of the PETACC-3 trial. Ann Oncol, 2015. 26(1): p. 126-32.

113. Roth, A.D., et al., Prognostic role of KRAS and BRAF in stage II and III resected colon cancer: results of the translational study on the PETACC-3, EORTC 40993, SAKK 60-00 trial. J Clin Oncol, 2010. 28(3): p. 466-74.

114. Fink, D., et al., In vitro and in vivo resistance to cisplatin in cells that have lost DNA mismatch repair. Cancer Res, 1997. 57(10): p. 1841-5. 
115. Aebi, S., et al., Loss of DNA mismatch repair in acquired resistance to cisplatin. Cancer Res, 1996. 56(13): p. 3087-90.

116. Fink, D., et al., The role of DNA mismatch repair in platinum drug resistance. Cancer Res, 1996. 56(21): p. 4881-6.

117. Marcelis, C.L., et al., Chemotherapy resistant ovarian cancer in carriers of an hMSH2 mutation? Fam Cancer, 2001. 1(2): p. 107-9.

118. Samimi, G., et al., Analysis of MLH1 and MSH2 expression in ovarian cancer before and after platinum drug-based chemotherapy. Clin Cancer Res, 2000. 6(4): p. 1415-21.

119. Honecker, F., et al., Microsatellite instability, mismatch repair deficiency, and BRAF mutation in treatment-resistant germ cell tumors. J Clin Oncol, 2009. 27(13): p. 2129-36.

120. Tougeron, D., et al., Efficacy of Adjuvant Chemotherapy in Colon Cancer With Microsatellite Instability: A Large Multicenter AGEO Study. J Natl Cancer Inst, 2016. 108(7).

121. Vilar, E., et al., Microsatellite instability due to $h M L H 1$ deficiency is associated with increased cytotoxicity to irinotecan in human colorectal cancer cell lines. $\mathrm{Br}$ J Cancer, 2008. 99(10): p. 1607-12.

122. Rodriguez, R., et al., Thymidine selectively enhances growth suppressive effects of camptothecin/irinotecan in MSI+ cells and tumors containing a mutation of MRE11. Clin Cancer Res, 2008. 14(17): p. 5476-83.

123. Fedier, A., et al., Resistance to topoisomerase poisons due to loss of DNA mismatch repair. Int J Cancer, 2001. 93(4): p. 571-6.

124. Jacob, S., et al., The role of the DNA mismatch repair system in the cytotoxicity of the topoisomerase inhibitors camptothecin and etoposide to human colorectal cancer cells. Cancer Res, 2001. 61(17): p. 6555-62.

125. Bertagnolli, M.M., et al., Microsatellite instability predicts improved response to adjuvant therapy with irinotecan, fluorouracil, and leucovorin in stage III colon cancer: Cancer and Leukemia Group B Protocol 89803. J Clin Oncol, 2009. 27(11): p. 1814-21.

126. Fu, D., J.A. Calvo, and L.D. Samson, Balancing repair and tolerance of DNA damage caused by alkylating agents. Nat Rev Cancer, 2012. 12(2): p. 104-20.

127. Yip, S., et al., MSH6 mutations arise in glioblastomas during temozolomide therapy and mediate temozolomide resistance. Clin Cancer Res, 2009. 15(14): p. 4622-9.

128. Zhang, J., et al., Acquired resistance to temozolomide in glioma cell lines: molecular mechanisms and potential translational applications. Oncology, 2010. 78(2): p. 103-14.

129. Koi, M., et al., Human chromosome 3 corrects mismatch repair deficiency and microsatellite instability and reduces $N$-methyl-N'-nitro- $N$-nitrosoguanidine tolerance in colon tumor cells with homozygous hMLH1 mutation. Cancer Res, 1994. 54(16): p. 4308-12.

130. Giles, R.E., et al., Maternal inheritance of human mitochondrial DNA. Proc Natl Acad Sci U S A, 1980. 77(11): p. 6715-9.

131. Lightowlers, R.N., et al., Mammalian mitochondrial genetics: heredity, heteroplasmy and disease. Trends Genet, 1997. 13(11): p. 450-5.

132. Chatterjee, A., E. Mambo, and D. Sidransky, Mitochondrial DNA mutations in human cancer. Oncogene, 2006. 25(34): p. 4663-74.

133. Attardi, G. and G. Schatz, Biogenesis of mitochondria. Annu Rev Cell Biol, 1988. 4: p. 289-333. 
134. Schon, E.A., Mitochondrial genetics and disease. Trends Biochem Sci, 2000. 25(11): p. 555-60.

135. Smeitink, J., L. van den Heuvel, and S. DiMauro, The genetics and pathology of oxidative phosphorylation. Nat Rev Genet, 2001. 2(5): p. 342-52.

136. Zickermann, V., et al., Architecture of complex I and its implications for electron transfer and proton pumping. Biochim Biophys Acta, 2009. 1787(6): p. 574-83.

137. Watt, I.N., et al., Bioenergetic cost of making an adenosine triphosphate molecule in animal mitochondria. Proc Natl Acad Sci U S A, 2010. 107(39): p. 16823-7.

138. Schultz, B.E. and S.I. Chan, Structures and proton-pumping strategies of mitochondrial respiratory enzymes. Annu Rev Biophys Biomol Struct, 2001. 30: p. 23-65.

139. Sazanov, L.A., A giant molecular proton pump: structure and mechanism of respiratory complex I. Nat Rev Mol Cell Biol, 2015. 16(6): p. 375-88.

140. Baradaran, R., et al., Crystal structure of the entire respiratory complex I. Nature, 2013. 494(7438): p. 443-8.

141. Mitchell, P., Coupling of phosphorylation to electron and hydrogen transfer by a chemi-osmotic type of mechanism. Nature, 1961. 191: p. 144-8.

142. Galkin, A.S., V.G. Grivennikova, and A.D. Vinogradov, -->H+/2e- stoichiometry in $N A D H$-quinone reductase reactions catalyzed by bovine heart submitochondrial particles. FEBS Lett, 1999. 451(2): p. 157-61.

143. Carroll, J., et al., Bovine complex I is a complex of 45 different subunits. J Biol Chem, 2006. 281(43): p. 32724-7.

144. Yip, C.Y., et al., Evolution of respiratory complex I: "supernumerary" subunits are present in the alpha-proteobacterial enzyme. J Biol Chem, 2011. 286(7): p. 502333.

145. Efremov, R.G. and L.A. Sazanov, The coupling mechanism of respiratory complex I - a structural and evolutionary perspective. Biochim Biophys Acta, 2012. 1817(10): p. 1785-95.

146. Sazanov, L.A., Respiratory complex I: mechanistic and structural insights provided by the crystal structure of the hydrophilic domain. Biochemistry, 2007. 46(9): p. 2275-88.

147. Hirst, J., et al., The nuclear encoded subunits of complex I from bovine heart mitochondria. Biochim Biophys Acta, 2003. 1604(3): p. 135-50.

148. Stojanovski, D., et al., Import of nuclear-encoded proteins into mitochondria. Exp Physiol, 2003. 88(1): p. 57-64.

149. Janssen, R.J., et al., Mitochondrial complex I: structure, function and pathology. J Inherit Metab Dis, 2006. 29(4): p. 499-515.

150. Friedrich, T. and B. Bottcher, The gross structure of the respiratory complex I: a Lego System. Biochim Biophys Acta, 2004. 1608(1): p. 1-9.

151. Hunte, C., V. Zickermann, and U. Brandt, Functional modules and structural basis of conformational coupling in mitochondrial complex I. Science, 2010. 329(5990): p. 448-51.

152. Zickermann, V., et al., Structural biology. Mechanistic insight from the crystal structure of mitochondrial complex I. Science, 2015. 347(6217): p. 44-9.

153. Efremov, R.G. and L.A. Sazanov, Structure of the membrane domain of respiratory complex I. Nature, 2011. 476(7361): p. 414-20.

154. Efremov, R.G., R. Baradaran, and L.A. Sazanov, The architecture of respiratory complex I. Nature, 2010. 465(7297): p. 441-5. 
155. Sazanov, L.A. and P. Hinchliffe, Structure of the hydrophilic domain of respiratory complex I from Thermus thermophilus. Science, 2006. 311(5766): p. 1430-6.

156. Mathiesen, C. and C. Hagerhall, Transmembrane topology of the NuoL, M and N subunits of NADH:quinone oxidoreductase and their homologues among membrane-bound hydrogenases and bona fide antiporters. Biochim Biophys Acta, 2002. 1556(2-3): p. 121-32.

157. Brandt, U., Energy converting NADH:quinone oxidoreductase (complex I). Annu Rev Biochem, 2006. 75: p. 69-92.

158. Mimaki, M., et al., Understanding mitochondrial complex I assembly in health and disease. Biochim Biophys Acta, 2012. 1817(6): p. 851-62.

159. Morgan, D.J. and L.A. Sazanov, Three-dimensional structure of respiratory complex I from Escherichia coli in ice in the presence of nucleotides. Biochim Biophys Acta, 2008. 1777(7-8): p. 711-8.

160. Zhu, J., K.R. Vinothkumar, and J. Hirst, Structure of mammalian respiratory complex I. Nature, 2016. 536(7616): p. 354-8.

161. Fiedorczuk, K., et al., Atomic structure of the entire mammalian mitochondrial complex I. Nature, 2016. 538(7625): p. 406-410.

162. Belevich, I., M.I. Verkhovsky, and M. Wikstrom, Proton-coupled electron transfer drives the proton pump of cytochrome c oxidase. Nature, 2006. 440(7085): p. 829-32.

163. Berrisford, J.M. and L.A. Sazanov, Structural basis for the mechanism of respiratory complex I. J Biol Chem, 2009. 284(43): p. 29773-83.

164. Scheffler, I.E., N. Yadava, and P. Potluri, Molecular genetics of complex Ideficient Chinese hamster cell lines. Biochim Biophys Acta, 2004. 1659(2-3): p. 160-71.

165. Yadava, N., et al., Species-specific and mutant MWFE proteins. Their effect on the assembly of a functional mammalian mitochondrial complex I. J Biol Chem, 2002. 277(24): p. 21221-30.

166. Remacle, C., et al., Eukaryotic complex I: functional diversity and experimental systems to unravel the assembly process. Mol Genet Genomics, 2008. 280(2): p. 93-110.

167. Triepels, R.H., et al., Respiratory chain complex I deficiency. Am J Med Genet, 2001. 106(1): p. 37-45.

168. Benit, P., et al., Large-scale deletion and point mutations of the nuclear NDUFV1 and NDUFS1 genes in mitochondrial complex I deficiency. Am J Hum Genet, 2001. 68(6): p. 1344-52.

169. Loeffen, J., et al., Mutations in the complex I NDUFS2 gene of patients with cardiomyopathy and encephalomyopathy. Ann Neurol, 2001. 49(2): p. 195-201.

170. Benit, P., et al., Mutant NDUFS3 subunit of mitochondrial complex I causes Leigh syndrome. J Med Genet, 2004. 41(1): p. 14-7.

171. Robinson, B.H., et al., Clinical presentation of mitochondrial respiratory chain defects in NADH-coenzyme $Q$ reductase and cytochrome oxidase: clues to pathogenesis of Leigh disease. J Pediatr, 1987. 110(2): p. 216-22.

172. Loeffen, J.L., et al., Isolated complex I deficiency in children: clinical, biochemical and genetic aspects. Hum Mutat, 2000. 15(2): p. 123-34.

173. Ugalde, C., et al., Differences in assembly or stability of complex I and other mitochondrial OXPHOS complexes in inherited complex I deficiency. Hum Mol Genet, 2004. 13(6): p. 659-67. 
174. Budde, S.M., et al., Combined enzymatic complex I and III deficiency associated with mutations in the nuclear encoded NDUFS4 gene. Biochem Biophys Res Commun, 2000. 275(1): p. 63-8.

175. Lamantea, E., et al., A novel nonsense mutation (Q352X) in the mitochondrial cytochrome $b$ gene associated with a combined deficiency of complexes I and III. Neuromuscul Disord, 2002. 12(1): p. 49-52.

176. Budde, S.M., et al., Clinical heterogeneity in patients with mutations in the NDUFS4 gene of mitochondrial complex I. J Inherit Metab Dis, 2003. 26(8): $p$. 813-5.

177. Schagger, H. and K. Pfeiffer, Supercomplexes in the respiratory chains of yeast and mammalian mitochondria. EMBO J, 2000. 19(8): p. 1777-83.

178. Schagger, H., Respiratory chain supercomplexes of mitochondria and bacteria. Biochim Biophys Acta, 2002. 1555(1-3): p. 154-9.

179. Gu, J., et al., The architecture of the mammalian respirasome. Nature, 2016. 537(7622): p. 639-43.

180. Francis, B.M., et al., Reduced levels of mitochondrial complex I subunit NDUFB8 and linked complex I + III oxidoreductase activity in the TgCRND8 mouse model of Alzheimer's disease. J Alzheimers Dis, 2014. 39(2): p. 347-55.

181. Kaelin, W.G., Jr., The concept of synthetic lethality in the context of anticancer therapy. Nat Rev Cancer, 2005. 5(9): p. 689-98.

182. de Murcia, J.M., et al., Requirement of poly(ADP-ribose) polymerase in recovery from DNA damage in mice and in cells. Proc Natl Acad Sci U S A, 1997. 94(14): p. 7303-7.

183. Lord, C.J. and A. Ashworth, PARP inhibitors: Synthetic lethality in the clinic. Science, 2017. 355(6330): p. 1152-1158.

184. Farmer, $\mathrm{H}$., et al., Targeting the DNA repair defect in BRCA mutant cells as a therapeutic strategy. Nature, 2005. 434(7035): p. 917-21.

185. Bryant, H.E., et al., Specific killing of BRCA2-deficient tumours with inhibitors of poly(ADP-ribose) polymerase. Nature, 2005. 434(7035): p. 913-7.

186. Guo, Z., et al., ATM activation by oxidative stress. Science, 2010. 330(6003): p. 517-21.

187. Martin, S.A., et al., Parallel high-throughput RNA interference screens identify PINK1 as a potential therapeutic target for the treatment of DNA mismatch repair-deficient cancers. Cancer Res, 2011. 71(5): p. 1836-48.

188. Martin, S.A., et al., Methotrexate induces oxidative DNA damage and is selectively lethal to tumour cells with defects in the DNA mismatch repair gene MSH2. EMBO Mol Med, 2009. 1(6-7): p. 323-37.

189. Frezza, C., et al., Haem oxygenase is synthetically lethal with the tumour suppressor fumarate hydratase. Nature, 2011. 477(7363): p. 225-8.

190. Boettcher, M., et al., High throughput synthetic lethality screen reveals a tumorigenic role of adenylate cyclase in fumarate hydratase-deficient cancer cells. BMC Genomics, 2014. 15: p. 158.

191. Szlosarek, P.W., et al., In vivo loss of expression of argininosuccinate synthetase in malignant pleural mesothelioma is a biomarker for susceptibility to arginine depletion. Clin Cancer Res, 2006. 12(23): p. 7126-31.

192. Feun, L.G., et al., Negative argininosuccinate synthetase expression in melanoma tumours may predict clinical benefit from arginine-depleting therapy with pegylated arginine deiminase. Br J Cancer, 2012. 106(9): p. 1481-5. 
193. Phillips, M.M., M.T. Sheaff, and P.W. Szlosarek, Targeting arginine-dependent cancers with arginine-degrading enzymes: opportunities and challenges. Cancer Res Treat, 2013. 45(4): p. 251-62.

194. Locke, M., et al., Inhibition of the Polyamine Synthesis Pathway Is Synthetically Lethal with Loss of Argininosuccinate Synthase 1. Cell Rep, 2016.

195. Menendez, J.A., et al., Metformin is synthetically lethal with glucose withdrawal in cancer cells. Cell Cycle, 2012. 11(15): p. 2782-92.

196. Chan, D.A. and A.J. Giaccia, Harnessing synthetic lethal interactions in anticancer drug discovery. Nat Rev Drug Discov, 2011. 10(5): p. 351-64.

197. Cheong, J.H., et al., Dual inhibition of tumor energy pathway by 2-deoxyglucose and metformin is effective against a broad spectrum of preclinical cancer models. Mol Cancer Ther, 2011. 10(12): p. 2350-62.

198. Kobayashi, H., et al., Identification of the determinants of 2-deoxyglucose sensitivity in cancer cells by shRNA library screening. Biochem Biophys Res Commun, 2015. 467(1): p. 121-7.

199. Gottlieb, E. and I.P. Tomlinson, Mitochondrial tumour suppressors: a genetic and biochemical update. Nat Rev Cancer, 2005. 5(11): p. 857-66.

200. Yan, H., et al., IDH1 and IDH2 mutations in gliomas. N Engl J Med, 2009. 360(8): p. 765-73.

201. King, A., M.A. Selak, and E. Gottlieb, Succinate dehydrogenase and fumarate hydratase: linking mitochondrial dysfunction and cancer. Oncogene, 2006. 25(34): p. 4675-82.

202. Bridge, G., S. Rashid, and S.A. Martin, DNA mismatch repair and oxidative DNA damage: implications for cancer biology and treatment. Cancers (Basel), 2014. 6(3): p. 1597-614.

203. Gorrini, C., I.S. Harris, and T.W. Mak, Modulation of oxidative stress as an anticancer strategy. Nat Rev Drug Discov, 2013. 12(12): p. 931-47.

204. Huie, R.E. and S. Padmaja, The reaction of no with superoxide. Free Radic Res Commun, 1993. 18(4): p. 195-9.

205. Dizdaroglu, M., Oxidatively induced DNA damage: mechanisms, repair and disease. Cancer Lett, 2012. 327(1-2): p. 26-47.

206. Quinlan, C.L., et al., The 2-oxoacid dehydrogenase complexes in mitochondria can produce superoxide/hydrogen peroxide at much higher rates than complex I. J Biol Chem, 2014. 289(12): p. 8312-25.

207. Sabharwal, S.S. and P.T. Schumacker, Mitochondrial ROS in cancer: initiators, amplifiers or an Achilles' heel? Nat Rev Cancer, 2014. 14(11): p. 709-21.

208. Gardner, P.R., Superoxide-driven aconitase FE-S center cycling. Biosci Rep, 1997. 17(1): p. 33-42.

209. Storr, S.J., C.M. Woolston, and S.G. Martin, Base excision repair, the redox environment and therapeutic implications. Curr Mol Pharmacol, 2012. 5(1): p. 88101.

210. Halliwell, B. and J.M.C. Gutteridge, Free radicals in biology and medicine. 4th ed. 2007, Oxford ; New York: Oxford University Press. xxxvi, 851 p., 8 p. of plates.

211. Karihtala, P. and Y. Soini, Reactive oxygen species and antioxidant mechanisms in human tissues and their relation to malignancies. APMIS, 2007. 115(2): p. 81103.

212. Valko, M., et al., Free radicals, metals and antioxidants in oxidative stressinduced cancer. Chem Biol Interact, 2006. 160(1): p. 1-40.

213. Steenken, S., Structure, acid/base properties and transformation reactions of purine radicals. Free Radic Res Commun, 1989. 6(2-3): p. 117-20. 
214. Cadet, J. and J.R. Wagner, DNA base damage by reactive oxygen species, oxidizing agents, and UV radiation. Cold Spring Harb Perspect Biol, 2013. 5(2).

215. Theruvathu, J.A., et al., Fenton chemistry of 1,3-dimethyluracil. J Am Chem Soc, 2001. 123(37): p. 9007-14.

216. Kamiya, H., Mutagenic potentials of damaged nucleic acids produced by reactive oxygen/nitrogen species: approaches using synthetic oligonucleotides and nucleotides: survey and summary. Nucleic Acids Res, 2003. 31(2): p. 517-31.

217. Kalam, M.A., et al., Genetic effects of oxidative DNA damages: comparative mutagenesis of the imidazole ring-opened formamidopyrimidines (Fapy lesions) and 8-oxo-purines in simian kidney cells. Nucleic Acids Res, 2006. 34(8): p. 2305-15.

218. Wood, M.L., et al., Mechanistic studies of ionizing radiation and oxidative mutagenesis: genetic effects of a single 8-hydroxyguanine (7-hydro-8oxoguanine) residue inserted at a unique site in a viral genome. Biochemistry, 1990. 29(30): p. 7024-32.

219. Greenberg, M.M., In vitro and in vivo effects of oxidative damage to deoxyguanosine. Biochem Soc Trans, 2004. 32(Pt 1): p. 46-50.

220. Kuchino, Y., et al., Misreading of DNA templates containing 8hydroxydeoxyguanosine at the modified base and at adjacent residues. Nature, 1987. 327(6117): p. 77-9.

221. Shibutani, S., M. Takeshita, and A.P. Grollman, Insertion of specific bases during DNA synthesis past the oxidation-damaged base 8-oxodG. Nature, 1991. 349(6308): p. 431-4.

222. Guschlbauer, W., et al., Structure and in vitro replication of DNA templates containing 7,8-dihydro-8-oxoadenine. Nucleic Acids Res, 1991. 19(8): p. 1753-8.

223. Tan, X., A.P. Grollman, and S. Shibutani, Comparison of the mutagenic properties of 8-oxo-7,8-dihydro-2'-deoxyadenosine and 8-oxo-7,8-dihydro-2'deoxyguanosine DNA lesions in mammalian cells. Carcinogenesis, 1999. 20(12): p. 2287-92.

224. Hayes, R.C., et al., Oxidative damage in DNA. Lack of mutagenicity by thymine glycol lesions. J Mol Biol, 1988. 201(2): p. 239-46.

225. Hayes, R.C. and J.E. LeClerc, Sequence dependence for bypass of thymine glycols in DNA by DNA polymerase I. Nucleic Acids Res, 1986. 14(2): p. 104561.

226. Feig, D.I., L.C. Sowers, and L.A. Loeb, Reverse chemical mutagenesis: identification of the mutagenic lesions resulting from reactive oxygen speciesmediated damage to DNA. Proc Natl Acad Sci U S A, 1994. 91(14): p. 6609-13.

227. Greenman, C., et al., Patterns of somatic mutation in human cancer genomes. Nature, 2007. 446(7132): p. 153-8.

228. Olivier, M., M. Hollstein, and P. Hainaut, TP53 mutations in human cancers: origins, consequences, and clinical use. Cold Spring Harb Perspect Biol, 2010. 2(1): p. a001008.

229. Konturek, P.C., S.J. Konturek, and T. Brzozowski, Gastric cancer and Helicobacter pylori infection. J Physiol Pharmacol, 2006. 57 Suppl 3: p. 51-65.

230. Seril, D.N., et al., Oxidative stress and ulcerative colitis-associated carcinogenesis: studies in humans and animal models. Carcinogenesis, 2003. 24(3): p. 353-362.

231. Kryston, T.B., et al., Role of oxidative stress and DNA damage in human carcinogenesis. Mutat Res, 2011. 711(1-2): p. 193-201. 
232. Matsui, A., et al., Increased formation of oxidative DNA damage, 8-hydroxy-2'deoxyguanosine, in human breast cancer tissue and its relationship to GSTP1 and COMT genotypes. Cancer Lett, 2000. 151(1): p. 87-95.

233. Oliva, M.R., et al., Genetic alterations and oxidative metabolism in sporadic colorectal tumors from a Spanish community. Mol Carcinog, 1997. 18(4): p. 23243.

234. Farinati, $\mathrm{F}$., et al., Oxidative DNA damage accumulation in gastric carcinogenesis. Gut, 1998. 42(3): p. 351-6.

235. Gackowski, D., et al., Persistent oxidative stress in colorectal carcinoma patients. Int J Cancer, 2002. 101(4): p. 395-7.

236. Olinski, R., et al., Oxidative DNA damage in cancer patients: a cause or a consequence of the disease development? Mutation Research/Fundamental and Molecular Mechanisms of Mutagenesis, 2003. 531(1-2): p. 177-190.

237. Malins, D.C., et al., The etiology of breast cancer. Characteristic alteration in hydroxyl radical-induced DNA base lesions during oncogenesis with potential for evaluating incidence risk. Cancer, 1993. 71(10): p. 3036-43.

238. Nowsheen, S., et al., Accumulation of oxidatively induced clustered DNA lesions in human tumor tissues. Mutat Res, 2009. 674(1-2): p. 131-6.

239. Sedelnikova, O.A., et al., Role of oxidatively induced DNA lesions in human pathogenesis. Mutat Res, 2010. 704(1-3): p. 152-9.

240. Mantovani, A., et al., Cancer-related inflammation. Nature, 2008. 454(7203): p. 436-44.

241. De Bont, R. and N. van Larebeke, Endogenous DNA damage in humans: a review of quantitative data. Mutagenesis, 2004. 19(3): p. 169-85.

242. Bolton, J.L. and G.R. Thatcher, Potential mechanisms of estrogen quinone carcinogenesis. Chem Res Toxicol, 2008. 21(1): p. 93-101.

243. Cadet, J., et al., Assessment of oxidative base damage to isolated and cellular DNA by HPLC-MS/MS measurement. Free Radic Biol Med, 2002. 33(4): p. 4419.

244. Klungland, A., et al., Accumulation of premutagenic DNA lesions in mice defective in removal of oxidative base damage. Proc Natl Acad Sci U S A, 1999. 96(23): p. 13300-5.

245. Georgakilas, A.G., Processing of DNA damage clusters in human cells: current status of knowledge. Mol Biosyst, 2008. 4(1): p. 30-5.

246. Demple, B. and L. Harrison, Repair of oxidative damage to DNA: enzymology and biology. Annu Rev Biochem, 1994. 63: p. 915-48.

247. Barzilai, A. and K. Yamamoto, DNA damage responses to oxidative stress. DNA Repair (Amst), 2004. 3(8-9): p. 1109-15.

248. Kazak, L., A. Reyes, and I.J. Holt, Minimizing the damage: repair pathways keep mitochondrial DNA intact. Nat Rev Mol Cell Biol, 2012. 13(10): p. 659-71.

249. Hegde, M.L., T.K. Hazra, and S. Mitra, Early steps in the DNA base excision/single-strand interruption repair pathway in mammalian cells. Cell Res, 2008. 18(1): p. 27-47.

250. Nakabeppu, Y., Regulation of intracellular localization of human MTH1, OGG1, and MYH proteins for repair of oxidative DNA damage. Prog Nucleic Acid Res Mol Biol, 2001. 68: p. 75-94.

251. Dunn, A.R., et al., Single Qdot-labeled glycosylase molecules use a wedge amino acid to probe for lesions while scanning along DNA. Nucleic Acids Res, 2011. 39(17): p. 7487-98. 
252. Slupphaug, G., et al., A nucleotide-flipping mechanism from the structure of human uracil-DNA glycosylase bound to DNA. Nature, 1996. 384(6604): p. 8792.

253. Garg, P., et al., Idling by DNA polymerase delta maintains a ligatable nick during lagging-strand DNA replication. Genes Dev, 2004. 18(22): p. 2764-73.

254. Frosina, G., et al., Two pathways for base excision repair in mammalian cells. J Biol Chem, 1996. 271(16): p. 9573-8.

255. Kim, Y.J. and D.M. Wilson, 3rd, Overview of base excision repair biochemistry. Curr Mol Pharmacol, 2012. 5(1): p. 3-13.

256. Lin, J.J. and A. Sancar, $A$ new mechanism for repairing oxidative damage to $D N A$ : (A)BC excinuclease removes AP sites and thymine glycols from DNA. Biochemistry, 1989. 28(20): p. 7979-84.

257. Reardon, J.T., et al., In vitro repair of oxidative DNA damage by human nucleotide excision repair system: possible explanation for neurodegeneration in xeroderma pigmentosum patients. Proc Natl Acad Sci U S A, 1997. 94(17): p. 9463-8.

258. Brooks, P.J., et al., The oxidative DNA lesion 8,5'-(S)-cyclo-2'-deoxyadenosine is repaired by the nucleotide excision repair pathway and blocks gene expression in mammalian cells. J Biol Chem, 2000. 275(29): p. 22355-62.

259. Kuraoka, l., et al., Removal of oxygen free-radical-induced 5',8-purine cyclodeoxynucleosides from DNA by the nucleotide excision-repair pathway in human cells. Proc Natl Acad Sci U S A, 2000. 97(8): p. 3832-7.

260. Friedberg, E.C., How nucleotide excision repair protects against cancer. Nat Rev Cancer, 2001. 1(1): p. 22-33.

261. Yokoi, M., et al., The xeroderma pigmentosum group $C$ protein complex XPCHR23B plays an important role in the recruitment of transcription factor IIH to damaged DNA. J Biol Chem, 2000. 275(13): p. 9870-5.

262. Araujo, S.J., E.A. Nigg, and R.D. Wood, Strong functional interactions of TFIIH with $X P C$ and $X P G$ in human DNA nucleotide excision repair, without a preassembled repairosome. Mol Cell Biol, 2001. 21(7): p. 2281-91.

263. Wakasugi, M., et al., Damaged DNA-binding protein DDB stimulates the excision of cyclobutane pyrimidine dimers in vitro in concert with XPA and replication protein A. J Biol Chem, 2001. 276(18): p. 15434-40.

264. Saldivar, J.S., et al., Nucleotide excision repair pathway review l: implications in ovarian cancer and platinum sensitivity. Gynecol Oncol, 2007. 107(1 Suppl 1): p. S56-71.

265. Boiteux, S. and J.P. Radicella, The human OGG1 gene: structure, functions, and its implication in the process of carcinogenesis. Arch Biochem Biophys, 2000. 377(1): p. 1-8.

266. Mambo, E., et al., Oxidized guanine lesions and hOgg1 activity in lung cancer. Oncogene, 2005. 24(28): p. 4496-508.

267. Chan, M.K., et al., Targeted deletion of the genes encoding NTH1 and NEIL1 DNA N-glycosylases reveals the existence of novel carcinogenic oxidative damage to DNA. DNA Repair (Amst), 2009. 8(7): p. 786-94.

268. Xanthoudakis, S., et al., The redox/DNA repair protein, Ref-1, is essential for early embryonic development in mice. Proc Natl Acad Sci U S A, 1996. 93(17): $p$. 8919-23.

269. Meira, L.B., et al., Heterozygosity for the mouse Apex gene results in phenotypes associated with oxidative stress. Cancer Res, 2001. 61(14): p. 5552-7. 
270. Huamani, J., et al., Spontaneous mutagenesis is enhanced in Apex heterozygous mice. Mol Cell Biol, 2004. 24(18): p. 8145-53.

271. Kelley, M.R., et al., Elevated and altered expression of the multifunctional DNA base excision repair and redox enzyme Ape1/ref-1 in prostate cancer. Clin Cancer Res, 2001. 7(4): p. 824-30.

272. Naidu, M.D., et al., Radiation resistance in glioma cells determined by DNA damage repair activity of Ape1/Ref-1. J Radiat Res, 2010. 51(4): p. 393-404.

273. Robertson, K.A., et al., Altered expression of Ape1/ref-1 in germ cell tumors and overexpression in NT2 cells confers resistance to bleomycin and radiation. Cancer Res, 2001. 61(5): p. 2220-5.

274. Agachan, B., et al., Apurinic/apyrimidinic endonuclease (APE1) gene polymorphisms and lung cancer risk in relation to tobacco smoking. Anticancer Res, 2009. 29(6): p. 2417-20.

275. Li, C., et al., Genetic variants of the ADPRT, XRCC1 and APE1 genes and risk of cutaneous melanoma. Carcinogenesis, 2006. 27(9): p. 1894-901.

276. Illuzzi, J.L., et al., Functional assessment of population and tumor-associated APE1 protein variants. PLoS One, 2013. 8(6): p. e65922.

277. Turnbull, C., et al., Genome-wide association study identifies five new breast cancer susceptibility loci. Nat Genet, 2010. 42(6): p. 504-7.

278. Dobashi, Y., et al., DNA polymerase beta gene mutation in human prostate cancer. Cancer Res, 1994. 54(11): p. 2827-9.

279. Kubota, Y., et al., Reduced fidelity of DNA synthesis in cell extracts from chemically induced primary thymic lymphomas of mice. Cancer Res, 1995. 55(17): p. 3777-80.

280. Matsuzaki, J., et al., DNA polymerase beta gene mutations in human bladder cancer. Mol Carcinog, 1996. 15(1): p. 38-43.

281. Efrati, E., et al., "Action-at-a-distance" mutagenesis. 8-oxo-7, 8-dihydro-2'deoxyguanosine causes base substitution errors at neighboring template sites when copied by DNA polymerase beta. J Biol Chem, 1999. 274(22): p. 15920-6.

282. Bhattacharyya, N., et al., Variant forms of DNA polymerase beta in primary lung carcinomas. DNA Cell Biol, 1999. 18(7): p. 549-54.

283. Li, D., et al., Effects of base excision repair gene polymorphisms on pancreatic cancer survival. Int J Cancer, 2007. 120(8): p. 1748-54.

284. Brierley, D.J. and S.A. Martin, Oxidative stress and the DNA mismatch repair pathway. Antioxid Redox Signal, 2013. 18(18): p. 2420-8.

285. Russo, M.T., et al., Different DNA repair strategies to combat the threat from 8oxoguanine. Mutat Res, 2007. 614(1-2): p. 69-76.

286. DeWeese, T.L., et al., Mouse embryonic stem cells carrying one or two defective Msh2 alleles respond abnormally to oxidative stress inflicted by low-level radiation. Proc Natl Acad Sci U S A, 1998. 95(20): p. 11915-20.

287. Colussi, C., et al., The mammalian mismatch repair pathway removes DNA 8oxodGMP incorporated from the oxidized dNTP pool. Curr Biol, 2002. 12(11): p. 912-8.

288. Wang, G., et al., The Helicobacter pylori MutS protein confers protection from oxidative DNA damage. Mol Microbiol, 2005. 58(1): p. 166-76.

289. Mazurek, A., M. Berardini, and R. Fishel, Activation of human MutS homologs by 8-oxo-guanine DNA damage. J Biol Chem, 2002. 277(10): p. 8260-6.

290. Ni, T.T., G.T. Marsischky, and R.D. Kolodner, MSH2 and MSH6 are required for removal of adenine misincorporated opposite 8-oxo-guanine in S. cerevisiae. Mol Cell, 1999. 4(3): p. 439-44. 
291. Gu, Y., et al., Human MutY homolog, a DNA glycosylase involved in base excision repair, physically and functionally interacts with mismatch repair proteins human MutS homolog 2/human MutS homolog 6. J Biol Chem, 2002. 277(13): p. 11135-42.

292. Glaab, W.E., R.B. Hill, and T.R. Skopek, Suppression of spontaneous and hydrogen peroxide-induced mutagenesis by the antioxidant ascorbate in mismatch repair-deficient human colon cancer cells. Carcinogenesis, 2001. 22(10): p. 1709-13.

293. Hardman, R.A., C.A. Afshari, and J.C. Barrett, Involvement of Mammalian MLH1 in the Apoptotic Response to Peroxide-induced Oxidative Stress. Cancer Research, 2001. 61(4): p. 1392-1397.

294. Piao, J., et al., Mismatch repair deficient mice show susceptibility to oxidative stress-induced intestinal carcinogenesis. Int J Biol Sci, 2013. 10(1): p. 73-9.

295. Stuart, J.A., et al., Localization of mitochondrial DNA base excision repair to an inner membrane-associated particulate fraction. Nucleic Acids Res, 2005. 33(12): p. 3722-32.

296. Longley, M.J., et al., Identification of 5'-deoxyribose phosphate lyase activity in human DNA polymerase gamma and its role in mitochondrial base excision repair in vitro. Proc Natl Acad Sci U S A, 1998. 95(21): p. 12244-8.

297. Pohjoismaki, J.L., et al., Oxidative stress during mitochondrial biogenesis compromises mtDNA integrity in growing hearts and induces a global DNA repair response. Nucleic Acids Res, 2012. 40(14): p. 6595-607.

298. Rossi, M.N., et al., Mitochondrial localization of PARP-1 requires interaction with mitofilin and is involved in the maintenance of mitochondrial DNA integrity. J Biol Chem, 2009. 284(46): p. 31616-24.

299. Das, B.B., et al., Role of tyrosyl-DNA phosphodiesterase (TDP1) in mitochondria. Proc Natl Acad Sci U S A, 2010. 107(46): p. 19790-5.

300. Ahel, I., et al., The neurodegenerative disease protein aprataxin resolves abortive DNA ligation intermediates. Nature, 2006. 443(7112): p. 713-6.

301. Bacman, S.R., S.L. Williams, and C.T. Moraes, Intra- and inter-molecular recombination of mitochondrial DNA after in vivo induction of multiple doublestrand breaks. Nucleic Acids Res, 2009. 37(13): p. 4218-26.

302. Dmitrieva, N.I., D. Malide, and M.B. Burg, Mre11 is expressed in mammalian mitochondria where it binds to mitochondrial DNA. Am J Physiol Regul Integr Comp Physiol, 2011. 301(3): p. R632-40.

303. D'Aurelio, M., et al., Heterologous mitochondrial DNA recombination in human cells. Hum Mol Genet, 2004. 13(24): p. 3171-9.

304. Gilkerson, R.W., et al., Mitochondrial nucleoids maintain genetic autonomy but allow for functional complementation. J Cell Biol, 2008. 181(7): p. 1117-28.

305. Mita, S., et al., Recombination via flanking direct repeats is a major cause of large-scale deletions of human mitochondrial DNA. Nucleic Acids Res, 1990. 18(3): p. 561-7.

306. Shokolenko, I., et al., Oxidative stress induces degradation of mitochondrial DNA. Nucleic Acids Res, 2009. 37(8): p. 2539-48.

307. Ikeda, S. and K. Ozaki, Action of mitochondrial endonuclease G on DNA damaged by L-ascorbic acid, peplomycin, and cis-diamminedichloroplatinum (II). Biochem Biophys Res Commun, 1997. 235(2): p. 291-4.

308. Lin, J., C. Handschin, and B.M. Spiegelman, Metabolic control through the PGC1 family of transcription coactivators. Cell Metab, 2005. 1(6): p. 361-70. 
309. Kressler, D., et al., The PGC-1-related protein PERC is a selective coactivator of estrogen receptor alpha. J Biol Chem, 2002. 277(16): p. 13918-25.

310. Scarpulla, R.C., Metabolic control of mitochondrial biogenesis through the PGC-1 family regulatory network. Biochim Biophys Acta, 2011. 1813(7): p. 1269-78.

311. Leone, T.C., et al., PGC-1alpha deficiency causes multi-system energy metabolic derangements: muscle dysfunction, abnormal weight control and hepatic steatosis. PLoS Biol, 2005. 3(4): p. e101.

312. Horibata, Y. and H. Sugimoto, StarD7 mediates the intracellular trafficking of phosphatidylcholine to mitochondria. J Biol Chem, 2010. 285(10): p. 7358-65.

313. Calvo, S.E. and V.K. Mootha, The mitochondrial proteome and human disease. Annu Rev Genomics Hum Genet, 2010. 11: p. 25-44.

314. Shutt, T.E. and G.S. Shadel, A compendium of human mitochondrial gene expression machinery with links to disease. Environ Mol Mutagen, 2010. 51(5): p. 360-79.

315. Pagliarini, D.J., et al., A mitochondrial protein compendium elucidates complex I disease biology. Cell, 2008. 134(1): p. 112-23.

316. Meirhaeghe, A., et al., Characterization of the human, mouse and rat PGC1 beta (peroxisome-proliferator-activated receptor-gamma co-activator 1 beta) gene in vitro and in vivo. Biochem J, 2003. 373(Pt 1): p. 155-65.

317. Lin, J., et al., PGC-1beta in the regulation of hepatic glucose and energy metabolism. J Biol Chem, 2003. 278(33): p. 30843-8.

318. St-Pierre, J., et al., Bioenergetic analysis of peroxisome proliferator-activated receptor gamma coactivators 1alpha and 1beta (PGC-1alpha and PGC-1beta) in muscle cells. J Biol Chem, 2003. 278(29): p. 26597-603.

319. Scarpulla, R.C., Nuclear control of respiratory chain expression by nuclear respiratory factors and PGC-1-related coactivator. Ann N Y Acad Sci, 2008. 1147: p. 321-34.

320. Gopalakrishnan, L. and R.C. Scarpulla, Differential regulation of respiratory chain subunits by a CREB-dependent signal transduction pathway. Role of cyclic AMP in cytochrome $c$ and COXIV gene expression. J Biol Chem, 1994. 269(1): p. 10513.

321. Marin-Garcia, J., Thyroid hormone and myocardial mitochondrial biogenesis. Vascul Pharmacol, 2010. 52(3-4): p. 120-30.

322. Connaughton, S., et al., Regulation of pyruvate dehydrogenase kinase isoform 4 (PDK4) gene expression by glucocorticoids and insulin. Mol Cell Endocrinol, 2010. 315(1-2): p. 159-67.

323. Hock, M.B. and A. Kralli, Transcriptional control of mitochondrial biogenesis and function. Annu Rev Physiol, 2009. 71: p. 177-203.

324. Klinge, C.M., Estrogenic control of mitochondrial function and biogenesis. J Cell Biochem, 2008. 105(6): p. 1342-51.

325. Jones, A.W., et al., PGC-1 family coactivators and cell fate: roles in cancer, neurodegeneration, cardiovascular disease and retrograde mitochondria-nucleus signalling. Mitochondrion, 2012. 12(1): p. 86-99.

326. Bonawitz, N.D., D.A. Clayton, and G.S. Shadel, Initiation and beyond: multiple functions of the human mitochondrial transcription machinery. Mol Cell, 2006. 24(6): p. 813-25.

327. Scarpulla, R.C., Nuclear control of respiratory gene expression in mammalian cells. J Cell Biochem, 2006. 97(4): p. 673-83.

328. Goffart, S. and R.J. Wiesner, Regulation and co-ordination of nuclear gene expression during mitochondrial biogenesis. Exp Physiol, 2003. 88(1): p. 33-40. 
329. Evans, M.J. and R.C. Scarpulla, Interaction of nuclear factors with multiple sites in the somatic cytochrome c promoter. Characterization of upstream NRF-1, ATF, and intron Sp1 recognition sequences. J Biol Chem, 1989. 264(24): p. 14361-8.

330. Virbasius, J.V., C.A. Virbasius, and R.C. Scarpulla, Identity of GABP with NRF-2, a multisubunit activator of cytochrome oxidase expression, reveals a cellular role for an ETS domain activator of viral promoters. Genes Dev, 1993. 7(3): p. 38092.

331. Chau, C.M., M.J. Evans, and R.C. Scarpulla, Nuclear respiratory factor 1 activation sites in genes encoding the gamma-subunit of ATP synthase, eukaryotic initiation factor 2 alpha, and tyrosine aminotransferase. Specific interaction of purified NRF-1 with multiple target genes. J Biol Chem, 1992. 267(10): p. 6999-7006.

332. Gugneja, S. and R.C. Scarpulla, Serine phosphorylation within a concise aminoterminal domain in nuclear respiratory factor 1 enhances DNA binding. J Biol Chem, 1997. 272(30): p. 18732-9.

333. Virbasius, J.V. and R.C. Scarpulla, Activation of the human mitochondrial transcription factor $A$ gene by nuclear respiratory factors: a potential regulatory link between nuclear and mitochondrial gene expression in organelle biogenesis. Proc Natl Acad Sci U S A, 1994. 91(4): p. 1309-13.

334. Gleyzer, N., K. Vercauteren, and R.C. Scarpulla, Control of mitochondrial transcription specificity factors (TFB1M and TFB2M) by nuclear respiratory factors (NRF-1 and NRF-2) and PGC-1 family coactivators. Mol Cell Biol, 2005. 25(4): p. 1354-66.

335. Scarpulla, R.C., Nuclear activators and coactivators in mammalian mitochondrial biogenesis. Biochim Biophys Acta, 2002. 1576(1-2): p. 1-14.

336. Braidotti, G., I.A. Borthwick, and B.K. May, Identification of regulatory sequences in the gene for 5-aminolevulinate synthase from rat. J Biol Chem, 1993. 268(2): p. 1109-17.

337. Takahashi, Y., et al., Characterization and identification of promoter elements in the mouse COX17 gene. Biochim Biophys Acta, 2002. 1574(3): p. 359-64.

338. Huo, L. and R.C. Scarpulla, Mitochondrial DNA instability and peri-implantation lethality associated with targeted disruption of nuclear respiratory factor 1 in mice. Mol Cell Biol, 2001. 21(2): p. 644-54.

339. Hoppeler, H. and M. Fluck, Plasticity of skeletal muscle mitochondria: structure and function. Med Sci Sports Exerc, 2003. 35(1): p. 95-104.

340. Ongwijitwat, S. and M.T. Wong-Riley, Is nuclear respiratory factor 2 a master transcriptional coordinator for all ten nuclear-encoded cytochrome c oxidase subunits in neurons? Gene, 2005. 360(1): p. 65-77.

341. Virbasius, J.V. and R.C. Scarpulla, Transcriptional activation through ETS domain binding sites in the cytochrome c oxidase subunit IV gene. Mol Cell Biol, 1991. 11(11): p. 5631-8.

342. Blesa, J.R., J.M. Hernandez, and J. Hernandez-Yago, NRF-2 transcription factor is essential in promoting human Tomm70 gene expression. Mitochondrion, 2004. 3(5): p. 251-9.

343. van der Bliek, A.M., Q. Shen, and S. Kawajiri, Mechanisms of mitochondrial fission and fusion. Cold Spring Harb Perspect Biol, 2013. 5(6).

344. D'Erchia, A.M., et al., Tissue-specific mtDNA abundance from exome data and its correlation with mitochondrial transcription, mass and respiratory activity. Mitochondrion, 2015. 20: p. 13-21. 
345. Hori, A., et al., Reactive oxygen species regulate DNA copy number in isolated yeast mitochondria by triggering recombination-mediated replication. Nucleic Acids Res, 2009. 37(3): p. 749-61.

346. Achanta, G., et al., Novel role of p53 in maintaining mitochondrial genetic stability through interaction with DNA Pol gamma. EMBO J, 2005. 24(19): p. 3482-92.

347. Wong, T.S., et al., Biophysical characterizations of human mitochondrial transcription factor $A$ and its binding to tumor suppressor p53. Nucleic Acids Res, 2009. 37(20): p. 6765-83.

348. Wong, T.S., et al., Physical and functional interactions between human mitochondrial single-stranded DNA-binding protein and tumour suppressor p53. Nucleic Acids Res, 2009. 37(2): p. 568-81.

349. Holt, S.M., et al., Compromised repair of clustered DNA damage in the human acute lymphoblastic leukemia MSH2-deficient NALM-6 cells. Mutat Res, 2009. 674(1-2): p. 123-30.

350. Taguchi, K., H. Motohashi, and M. Yamamoto, Molecular mechanisms of the Keap1-Nrf2 pathway in stress response and cancer evolution. Genes Cells, 2011. 16(2): p. 123-40.

351. Yamamoto, T., et al., Physiological significance of reactive cysteine residues of Keap1 in determining Nrf2 activity. Mol Cell Biol, 2008. 28(8): p. 2758-70.

352. Antelmann, H. and J.D. Helmann, Thiol-based redox switches and gene regulation. Antioxid Redox Signal, 2011. 14(6): p. 1049-63.

353. Sporn, M.B. and K.T. Liby, NRF2 and cancer: the good, the bad and the importance of context. Nat Rev Cancer, 2012. 12(8): p. 564-71.

354. Sasaki, H., et al., Electrophile response element-mediated induction of the cystine/glutamate exchange transporter gene expression. J Biol Chem, 2002. 277(47): p. 44765-71.

355. Arner, E.S. and A. Holmgren, Physiological functions of thioredoxin and thioredoxin reductase. Eur J Biochem, 2000. 267(20): p. 6102-9.

356. Gutteridge, J.M., Iron promoters of the Fenton reaction and lipid peroxidation can be released from haemoglobin by peroxides. FEBS Lett, 1986. 201(2): p. 291-5.

357. Alam, J., et al., Nrf2, a Cap'n'Collar transcription factor, regulates induction of the heme oxygenase-1 gene. J Biol Chem, 1999. 274(37): p. 26071-8.

358. Kops, G.J., et al., Forkhead transcription factor FOXO3a protects quiescent cells from oxidative stress. Nature, 2002. 419(6904): p. 316-21.

359. Nogueira, V., et al., Akt determines replicative senescence and oxidative or oncogenic premature senescence and sensitizes cells to oxidative apoptosis. Cancer Cell, 2008. 14(6): p. 458-70.

360. Kruiswijk, F., C.F. Labuschagne, and K.H. Vousden, p53 in survival, death and metabolic health: a lifeguard with a licence to kill. Nat Rev Mol Cell Biol, 2015. 16(7): p. 393-405.

361. McCord, J.M. and I. Fridovich, Superoxide dismutase. An enzymic function for erythrocuprein (hemocuprein). J Biol Chem, 1969. 244(22): p. 6049-55.

362. Landis, G.N. and J. Tower, Superoxide dismutase evolution and life span regulation. Mech Ageing Dev, 2005. 126(3): p. 365-79.

363. Mates, J.M., C. Perez-Gomez, and I. Nunez de Castro, Antioxidant enzymes and human diseases. Clin Biochem, 1999. 32(8): p. 595-603.

364. Budanov, A.V., et al., Regeneration of peroxiredoxins by p53-regulated sestrins, homologs of bacterial AhpD. Science, 2004. 304(5670): p. 596-600. 
365. St-Pierre, J., et al., Suppression of reactive oxygen species and neurodegeneration by the PGC-1 transcriptional coactivators. Cell, 2006. 127(2): p. 397-408.

366. Cowell, R.M., et al., Identification of novel targets for PGC-1alpha and histone deacetylase inhibitors in neuroblastoma cells. Biochem Biophys Res Commun, 2009. 379(2): p. 578-82.

367. Kukidome, D., et al., Activation of AMP-activated protein kinase reduces hyperglycemia-induced mitochondrial reactive oxygen species production and promotes mitochondrial biogenesis in human umbilical vein endothelial cells. Diabetes, 2006. 55(1): p. 120-7.

368. Mootha, V.K., et al., Integrated analysis of protein composition, tissue diversity, and gene regulation in mouse mitochondria. Cell, 2003. 115(5): p. 629-40.

369. http://www.agilent.com/en-us/promotions/xftechnologyoverview. How to run an XF Assay. 2016.

370. Zielonka, J., J. Vasquez-Vivar, and B. Kalyanaraman, Detection of 2hydroxyethidium in cellular systems: a unique marker product of superoxide and hydroethidine. Nat Protoc, 2008. 3(1): p. 8-21.

371. Illumina. Human mtDNA Genome Guide (15037958)

2016.

372. Venegas, V., et al., Real-time quantitative PCR analysis of mitochondrial DNA content. Curr Protoc Hum Genet, 2011. Chapter 19: p. Unit 197.

373. Fulda, S., L. Galluzzi, and G. Kroemer, Targeting mitochondria for cancer therapy. Nat Rev Drug Discov, 2010. 9(6): p. 447-64.

374. Cancer Genome Atlas, N., Comprehensive molecular characterization of human colon and rectal cancer. Nature, 2012. 487(7407): p. 330-7.

375. Boland, C.R. and A. Goel, Microsatellite instability in colorectal cancer. Gastroenterology, 2010. 138(6): p. 2073-2087 e3.

376. Fong, P.C., et al., Inhibition of poly(ADP-ribose) polymerase in tumors from BRCA mutation carriers. N Engl J Med, 2009. 361(2): p. 123-34.

377. Sun, Y., et al., A NADPH oxidase-dependent redox signaling pathway mediates the selective radiosensitization effect of parthenolide in prostate cancer cells. Cancer Res, 2010. 70(7): p. 2880-90.

378. Carlisi, D., et al., The oxygen radicals involved in the toxicity induced by parthenolide in MDA-MB-231 cells. Oncol Rep, 2014. 32(1): p. 167-72.

379. D'Anneo, A., et al., Parthenolide generates reactive oxygen species and autophagy in MDA-MB231 cells. A soluble parthenolide analogue inhibits tumour growth and metastasis in a xenograft model of breast cancer. Cell Death Dis, 2013. 4: p. e891.

380. Dickinson, A., et al., The regulation of mitochondrial DNA copy number in glioblastoma cells. Cell Death Differ, 2013. 20(12): p. 1644-53.

381. Curry, E.A., 3rd, et al., Phase I dose escalation trial of feverfew with standardized doses of parthenolide in patients with cancer. Invest New Drugs, 2004. 22(3): p. 299-305.

382. Stegmaier, K., Chemical genomics and combo therapy. Blood, 2010. 116(26): p. 5786-8.

383. Hassane, D.C., et al., Chemical genomic screening reveals synergism between parthenolide and inhibitors of the PI-3 kinase and mTOR pathways. Blood, 2010. 116(26): p. 5983-90. 
384. Simunek, T., et al., Anthracycline-induced cardiotoxicity: overview of studies examining the roles of oxidative stress and free cellular iron. Pharmacol Rep, 2009. 61(1): p. 154-71. 


\section{Appendix}

Appendix 1: Results for mitochondrial sequencing of MLH1 deficient (HCT116) and proficient cell lines (HCT116+chr3) 


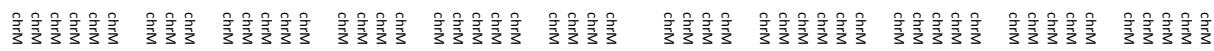

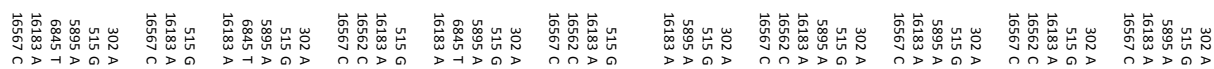

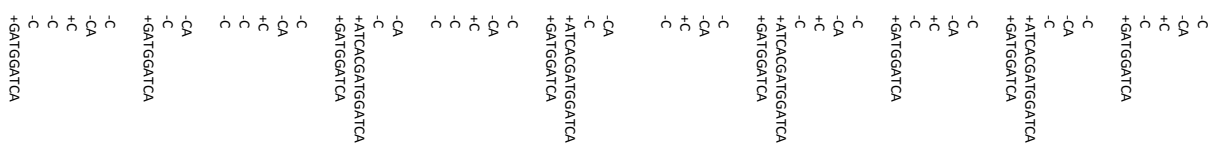

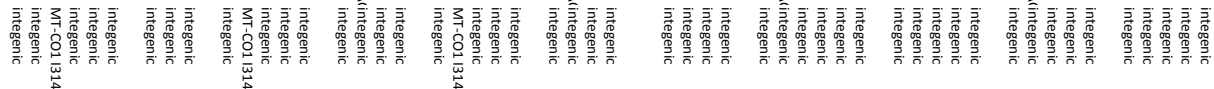

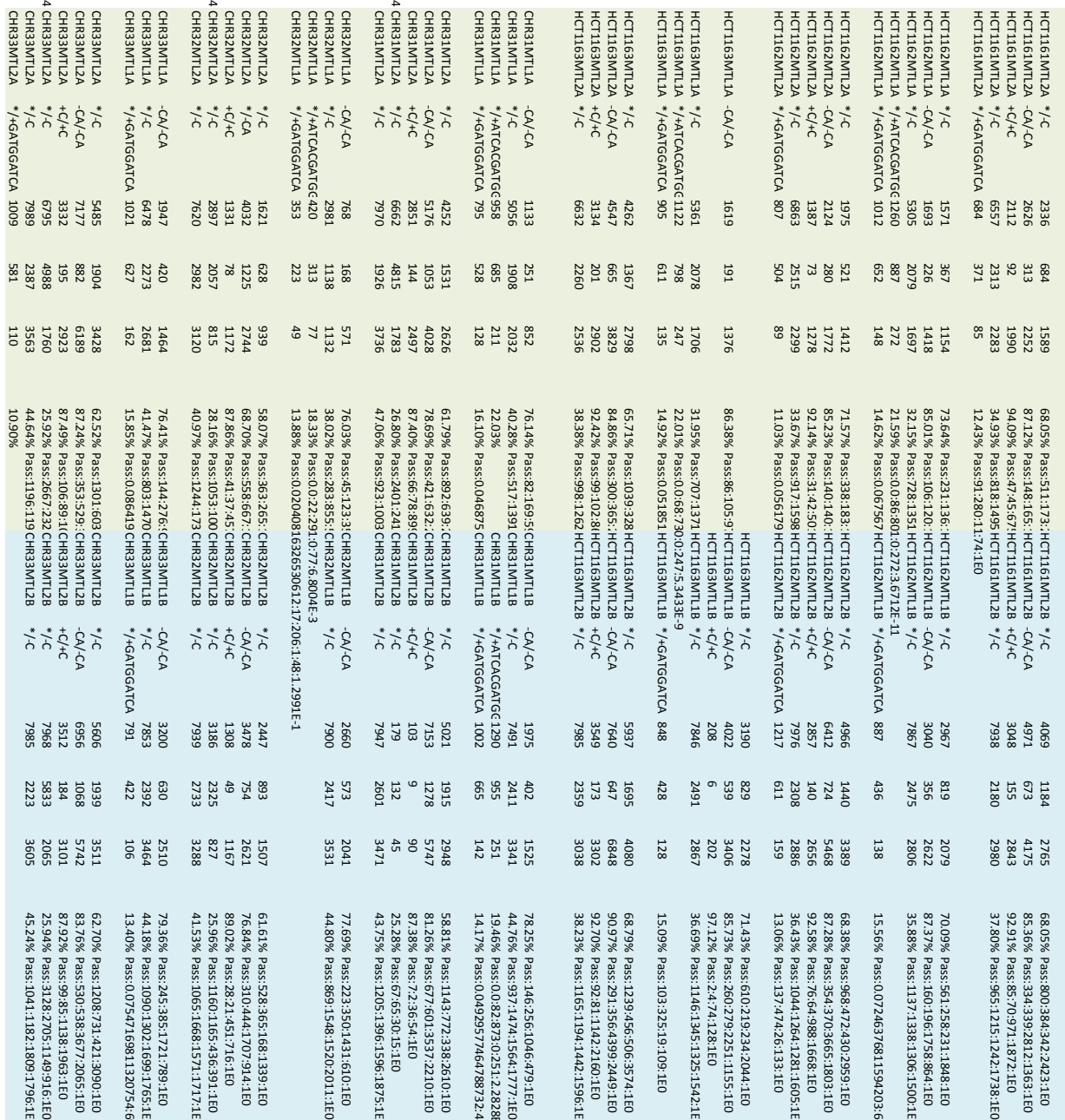

